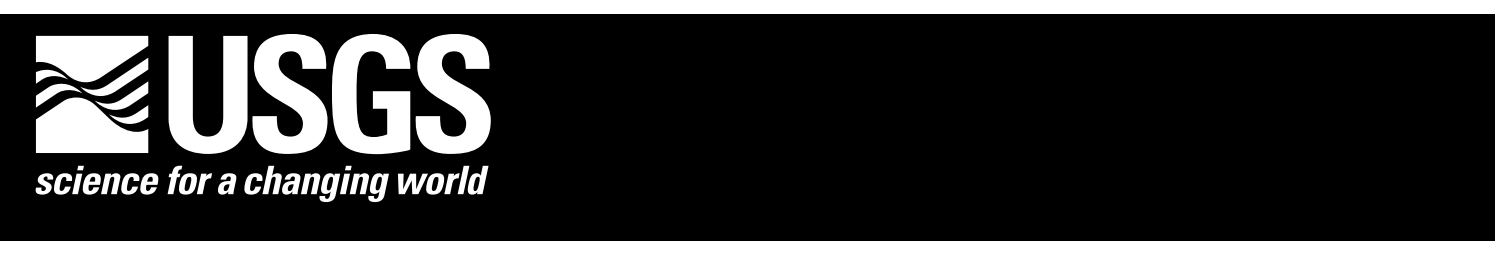

\title{
ARSENIC IN ROCKS AND STREAM SEDIMENTS OF THE CENTRAL APPALACHIAN BASIN, KENTUCKY
}

by Michele L.W. Tuttle, ${ }^{1}$ Martin B. Goldhaber, ${ }^{1}$ Leslie F. Ruppert, ${ }^{1}$ and James C. Hower ${ }^{2}$

Open-File Report 02-28

2002

This report is preliminary and has not been reviewed for conformity with U.S. Geological Survey editorial standards or with the North American Stratigraphic Code. Any use of trade, firm, or product names is for descriptive purposes only and does not imply endorsement by the U.S. Government.

\section{U.S. DEPARTMENT OF THE INTERIOR U.S. GEOLOGICAL SURVEY}

\footnotetext{
${ }^{1}$ U.S. Geological Survey, MS 973, Box 25046, Denver CO 80225

${ }^{2}$ University of Kentucky Center for Applied Energy Research, Lexington KY
} 


\section{TABLE OF CONTENTS}

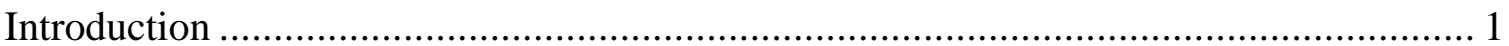

Energy Resources in the Kentucky Appalachian Basin............................................... 2

Arsenic in Kentucky Appalachian Basin Coal ..................................................... 2

Effect of regional structural features on spatial distribution of arsenic in eastern

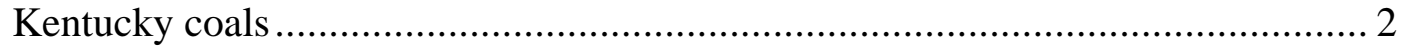

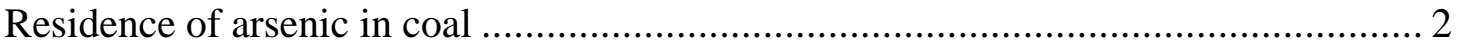

Arsenic in NURE samples from the Kentucky Appalachian Basin ............................... 10

Possible controls on arsenic in NURE samples from the Kentucky

Appalachian basin ................................................................................... 10

Arsenic in shale and coal collected in the Kentucky Appalachian basin......................... 13

Relation between iron and arsenic in the eastern Kentucky coal .............................. 14

Relation between iron and arsenic in the Devonian shale......................................... 16

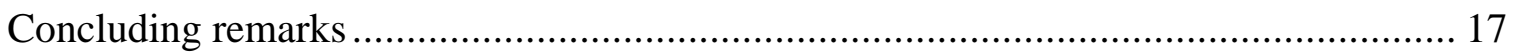

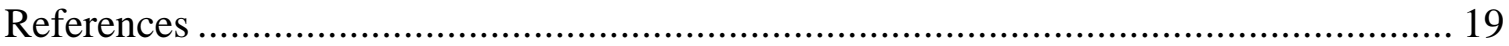

Appendix I—NURE stream sediment data (reanalyzed) ............................................ 20

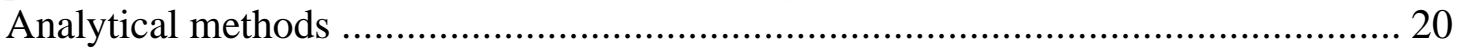

Stream sediment data …….......................................................................... 22

Appendix II —Coal and Devonian shale seimiquantitative XRF data .......................... 151

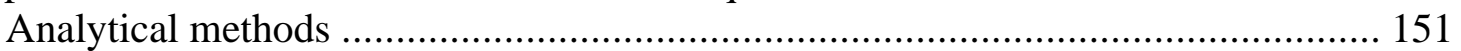

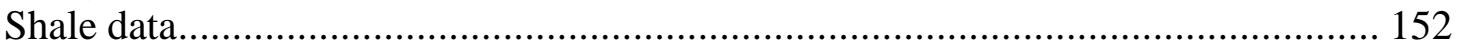

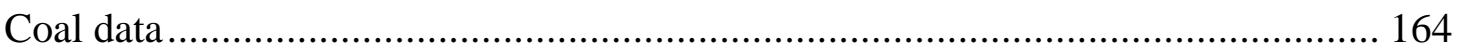

\section{FIGURES}

1. Map of Appalachian coal region in eastern USA ……........................................ 3

2. The resource map (A) shows coal resource by county. Photos (B-E) of the Kentucky

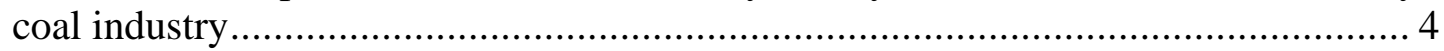

3. Histogram of arsenic concentrations in Eastern Kentucky coal ................................. 6

4. Spatial distribution of arsenic concentrations in east Kentucky coal........................... 7

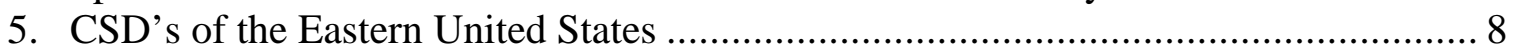

6. Cross plots of arsenic versus pyritic sulfur in drill=core, channel, and weathered

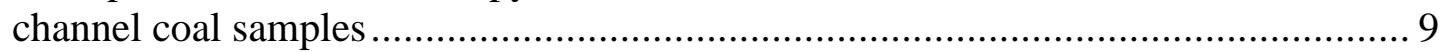

7. Histogram of arsenic concentrations in NURE stream sediments, Kentucky Appalachian Basin

8. Map showing generalized geology of the Kentucky Appalachian basin and arsenic concentrations in NURE stream sediments. ....................................................... 12

9. Cross plot of arsenic versus iron divided into regions within the basin...................... 13

10. Photos of shale and coal, Kentucky Appalachian basin.......................................... 15

11. Cross plot of iron versus arsenic in collected coal samples ...................................... 16

12. Cross plots of iron versus arsenic in shale outcrop and core samples ...................... 18 


\title{
ARSENIC IN ROCKS AND STREAM SEDIMENTS OF THE CENTRAL APPALACHIAN BASIN, KENTUCKY
}

\author{
By Michele L.W. Tuttle, Martin B. Goldhaber, Leslie F. Ruppert, and \\ James C. Hower
}

\section{INTRODUCTION}

Arsenic (As) enrichment in coal and stream sediments has been documented in the southern Appalachian basin (see Goldhaber and others, submitted) and is attributed to interaction of rocks and coal with metamorphic fluids generated during the Allegheny Orogeny (late Paleozoic). Similarly derived fluids are expected to affect the coal and in the Kentucky Appalachian Basin to the north as well. In addition, similar processes may have influenced the Devonian oil shale on the western margin of the basin. The major goals of this study are to determine the effect such fluids had on rocks in the Kentucky Appalachian basin (fig. 1), and to understand the geochemical processes that control trace-metal source, residence, and mobility within the basin. This report includes data presented in a poster at the USGS workshop on arsenic (February 21 and 22, 2001), new NURE stream sediment data ${ }^{3}$, and field data from a trip in April 2001. Although data for major and minor elements and all detectable trace metals are reported in the Appendices, the narrative of this report primarily focuses on arsenic.

The study is divided in to three phases. The first phase is completed and inventoried trace-metal concentrations in stream sediments throughout the Kentucky Appalachian basin. The distribution of arsenic concentrations was used to identify areas enriched in arsenic relative to estimated background values ${ }^{4}$. A summary of all chemical data from phase one is presented in Appendix I. The second phase is in progress and focuses on areas identified in phase one to have high concentrations of arsenic. Shale and coal from these regions were sampled for chemical and isotopic analyses and selective extractions to determine arsenic residence. Preliminary field data (including semi-quantitative X-ray fluorescence (XRF) data ${ }^{5}$ ) are presented in this report. The quantitative chemical and stable isotopic data currently being collected and analyzed will be used to determine source, residence and mobility of trace elements during weathering. Some preliminary results are presented in Tuttle and others (2001). The third phase (to begin 2002) will

\footnotetext{
${ }^{3}$ NURE stream sediment samples were retrieved from storage and reanalyzed in 2000.

${ }^{4}$ Estimated background values, as used in this report, represent average concentrations in stream sediments within the defined area (e.g. United States, Central Appalachian basin, etc.).

${ }^{5}$ XRF data were collected using a hand-held Niton spectrophotometer that measures surface concentrations (the use of the trade name Niton is for descriptive purposes only and does not imply endorsement by the U.S. Government).
} 
focus on specific watersheds to help identify processes that affect trace-element concentrations in streams and associated ecosystems.

\section{ENERGY RESOURCES IN THE KENTUCKY APPALACHIAN BASIN}

The Kentucky Appalachian basin has about 28 billion tons of recoverable coal today (fig. 2). There is about 190 billion barrels of synthetic oil in surface and near-surface Devonian oil shale within Kentucky (Matthews and others, 1981)--with a substantial portion along the western margin of the Appalachian basin. Although oil shale currently is not targeted for mining, most of the available coal in Kentucky will be mined.

\section{Arsenic in Kentucky Appalachian Basin Coal}

The geometric mean of arsenic in the eastern Kentucky coals is 11 ppm (U.S. Geological Survey Coal quality Database; Bragg and others, 1997). Over one-half of these coals have concentrations greater than the geometric mean concentration $(6.1 \mathrm{ppm})$ for all US coals in the database. Because the arsenic concentrations are log normally distributed (see fig. 3), all averages are given as geometric means.

\section{Effect of Regional Structural Features on Spatial Distribution of Arsenic in Eastern Kentucky Coals}

High arsenic concentrations (>24 ppm) in Kentucky coal appear to be oriented in lines trending northeast to southwest or northwest to southeast (fig. 4). The northeast to southwest trends are parallel to the regional trend of Appalachian fold and faults. The perpendicular trend (northwest to southeast) is parallel to mapped cross strike discontinuities (CSD; Coleman and others, 1988) (fig. 5). These CSD's and major Appalachian folds and faults could have focused hydrothermal fluids generated during orogenic processes into the Kentucky Appalachian Basin.

Eastern Kentucky coal rank increases to the southeast, probably due, in part, to a southeastward increase in paleogeothermal gradient (Hower and others, 1991). Flow of mineralizing hydrothermal fluids through the Central Appalachian Basin along CSD's from southeast to northwest is consistent with these paleogeothermal gradients.

\section{Residence of Arsenic in Coal}

Figure 6 shows arsenic concentrations plotted against pyritic sulfur concentrations for the drill-core, channel, and weathered channel samples in the USGS Coal Quality Database. The three plots all show a positive relation between the two variables indicating that pyrite is a likely candidate for the residence of the arsenic in the coal. A similar relation was found in all U.S. coals (Coleman and Bragg, 1990). The scatter in each of the plots likely reflects variations in the ratio of early diagenetic pyrite to epigenetic pyrite in the coal samples and variable amounts of siderite present in the coal. 


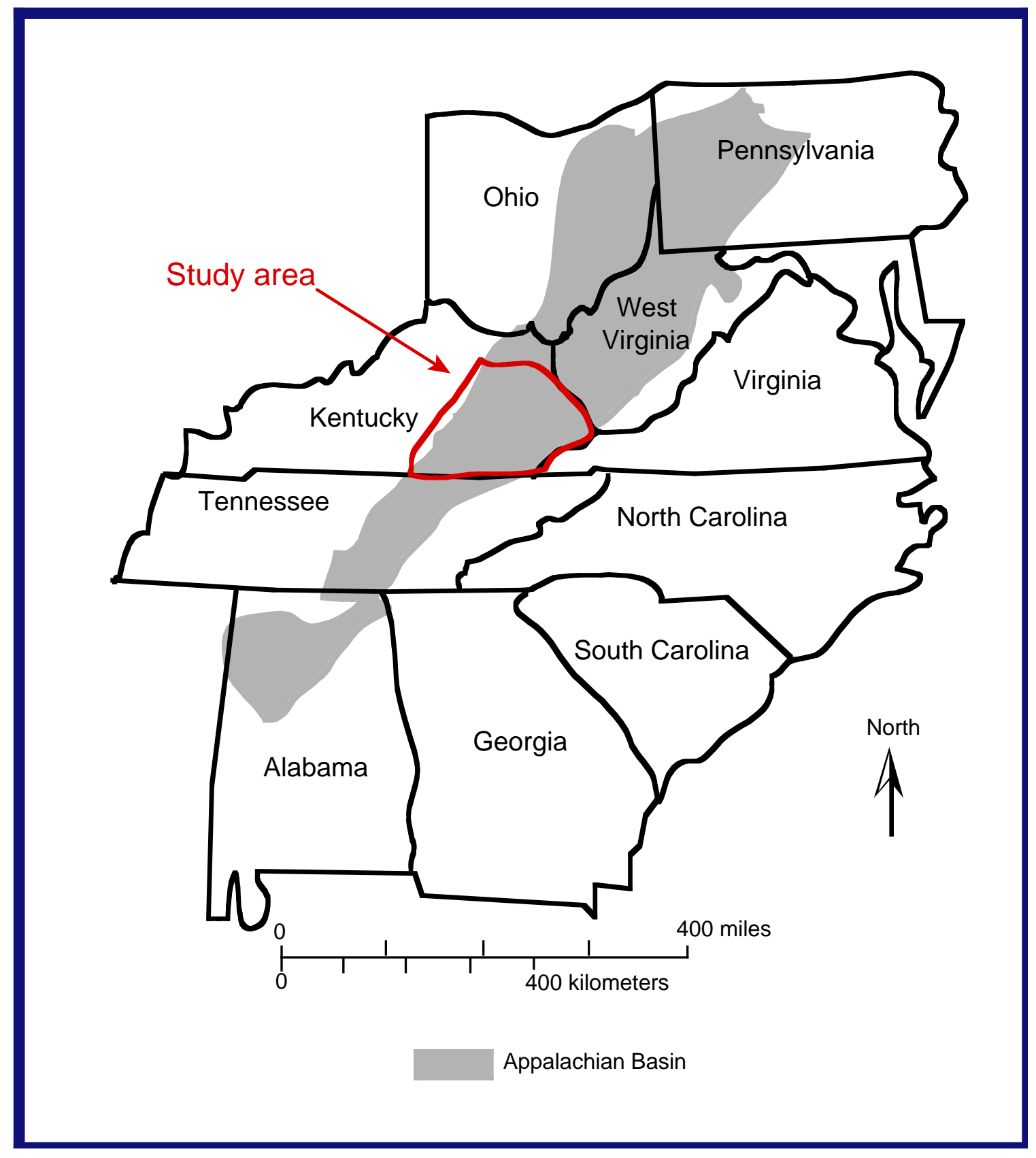

Figure 1. Map of the Appalachian Coal Region in Eastern USA showing location of study area. (modified from Britton and others, 1989) 


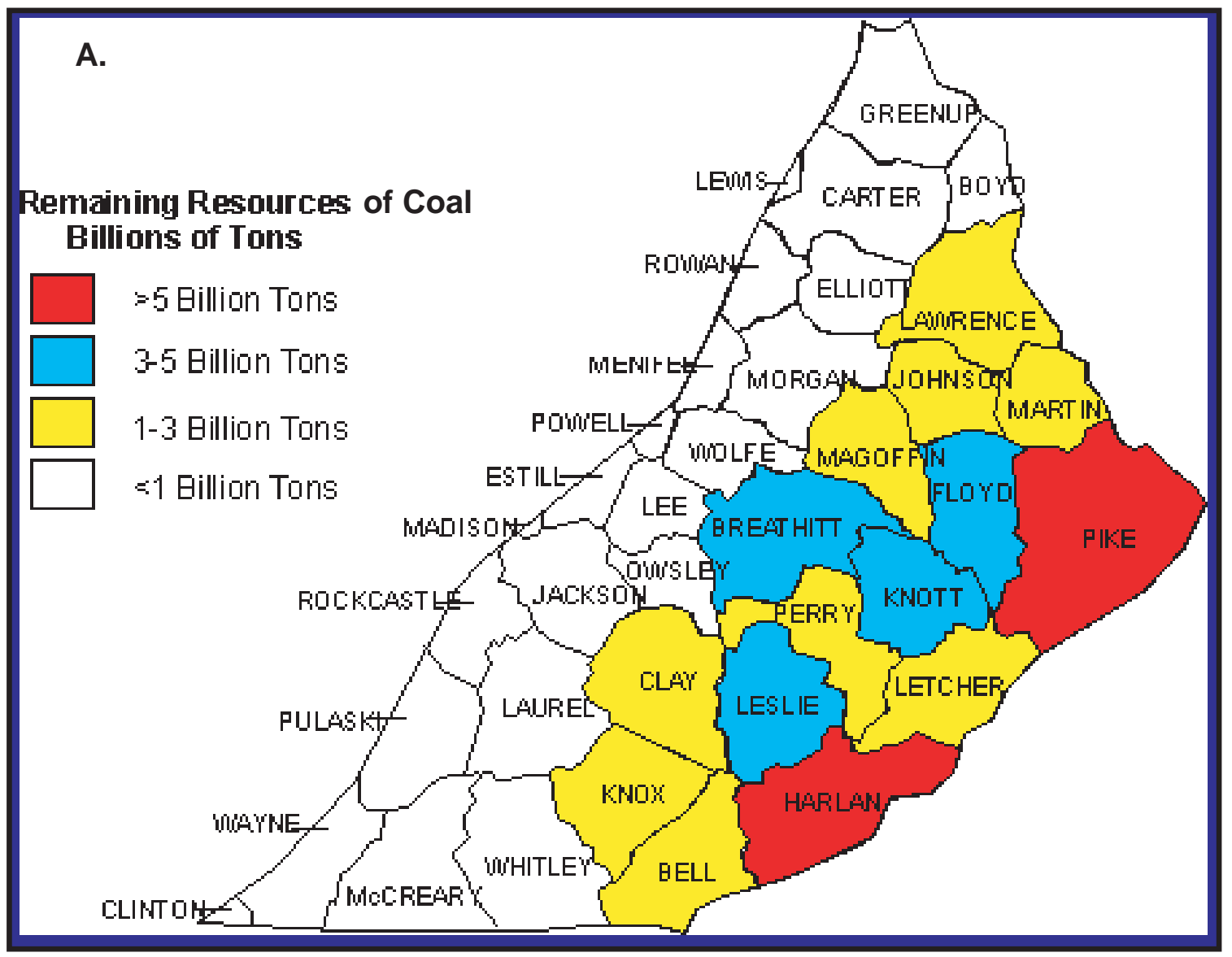



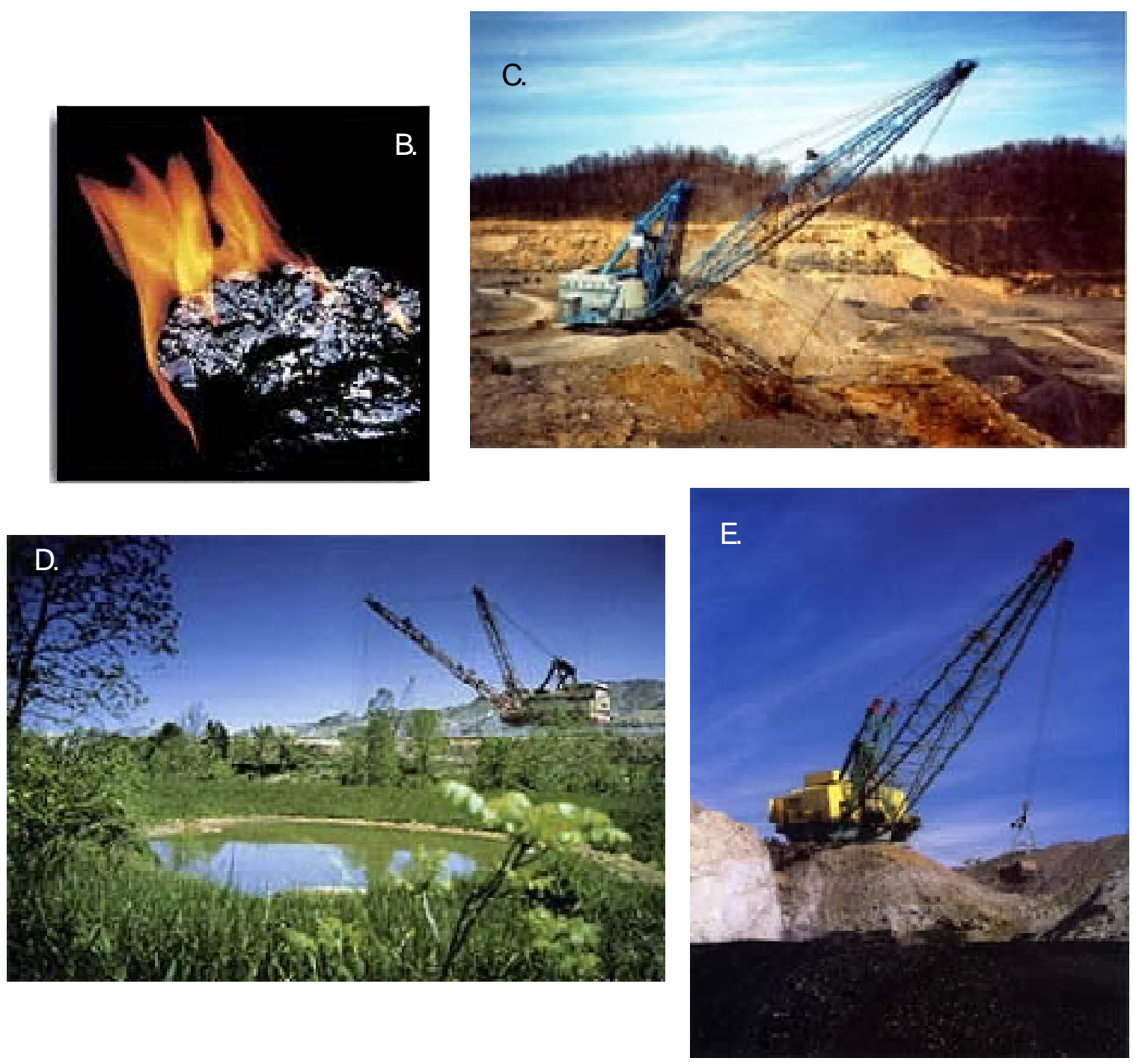

Figure 2. The resource map (A) shows eastern Kentucky coal resources by county. Photos (B-E) of the Kentucky coal industry and resource map were downloaded from

http://www.coaleducation.org/Ky Coal Facts/main coal facts.htm 


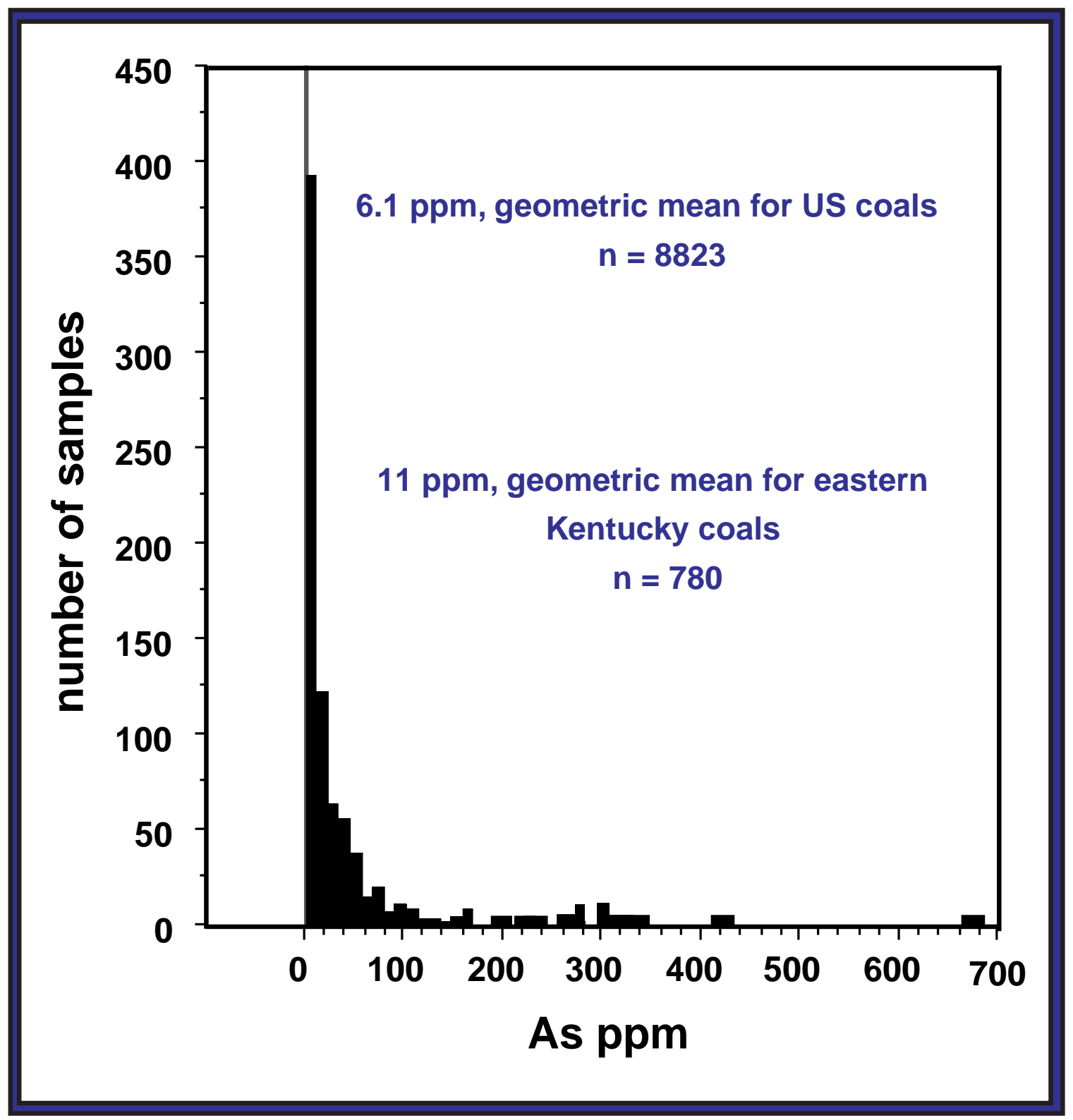

Figure 3. Histogram of arsenic concentrations (whole-rock basis) in Eastern Kentucky Coal (data from Coleman and Bragg, 1990; Bragg and others, 1997). 


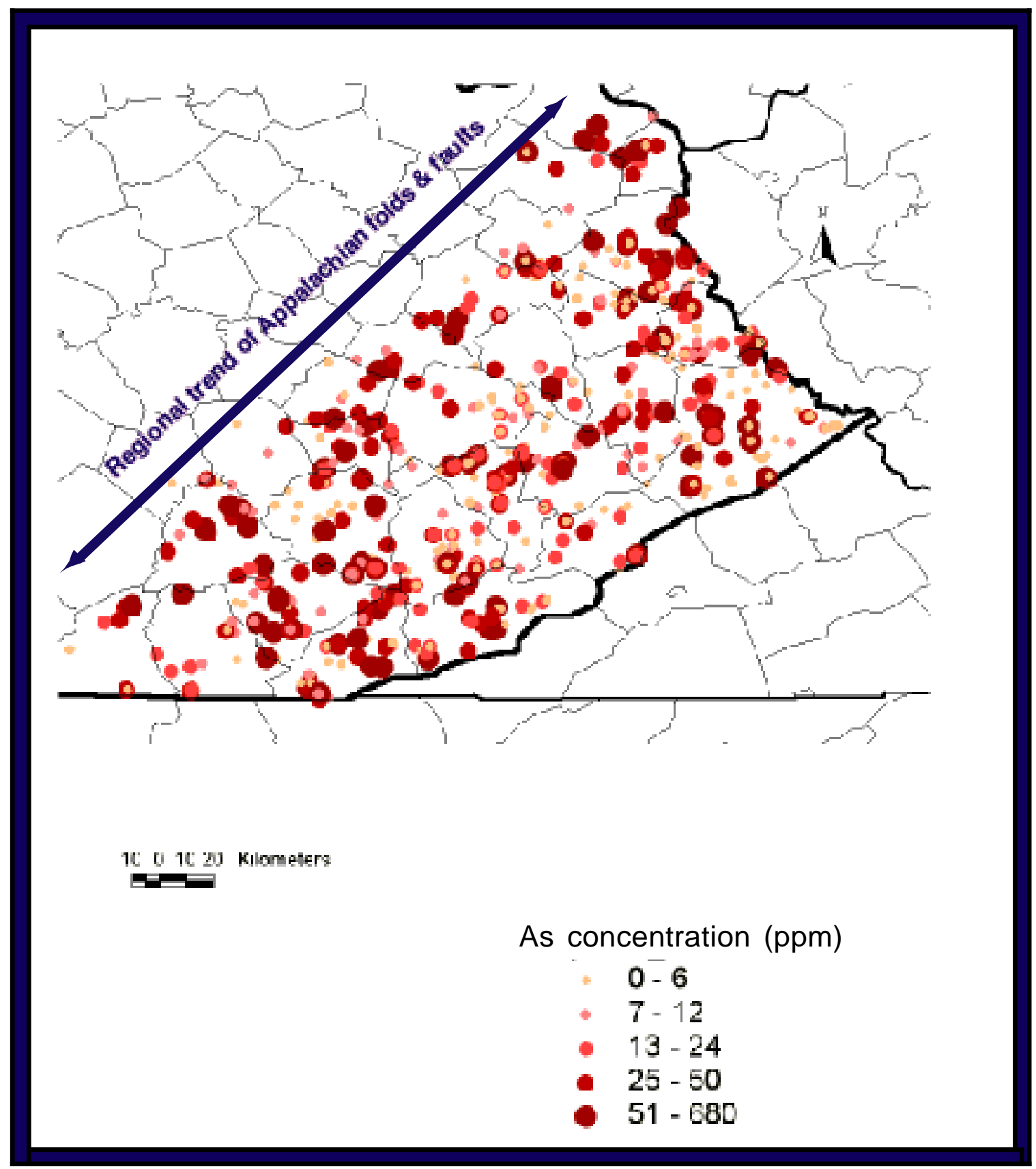

Figure 4. Spatial distribution of arsenic concentrations (ppm) in east Kentucky coal. 


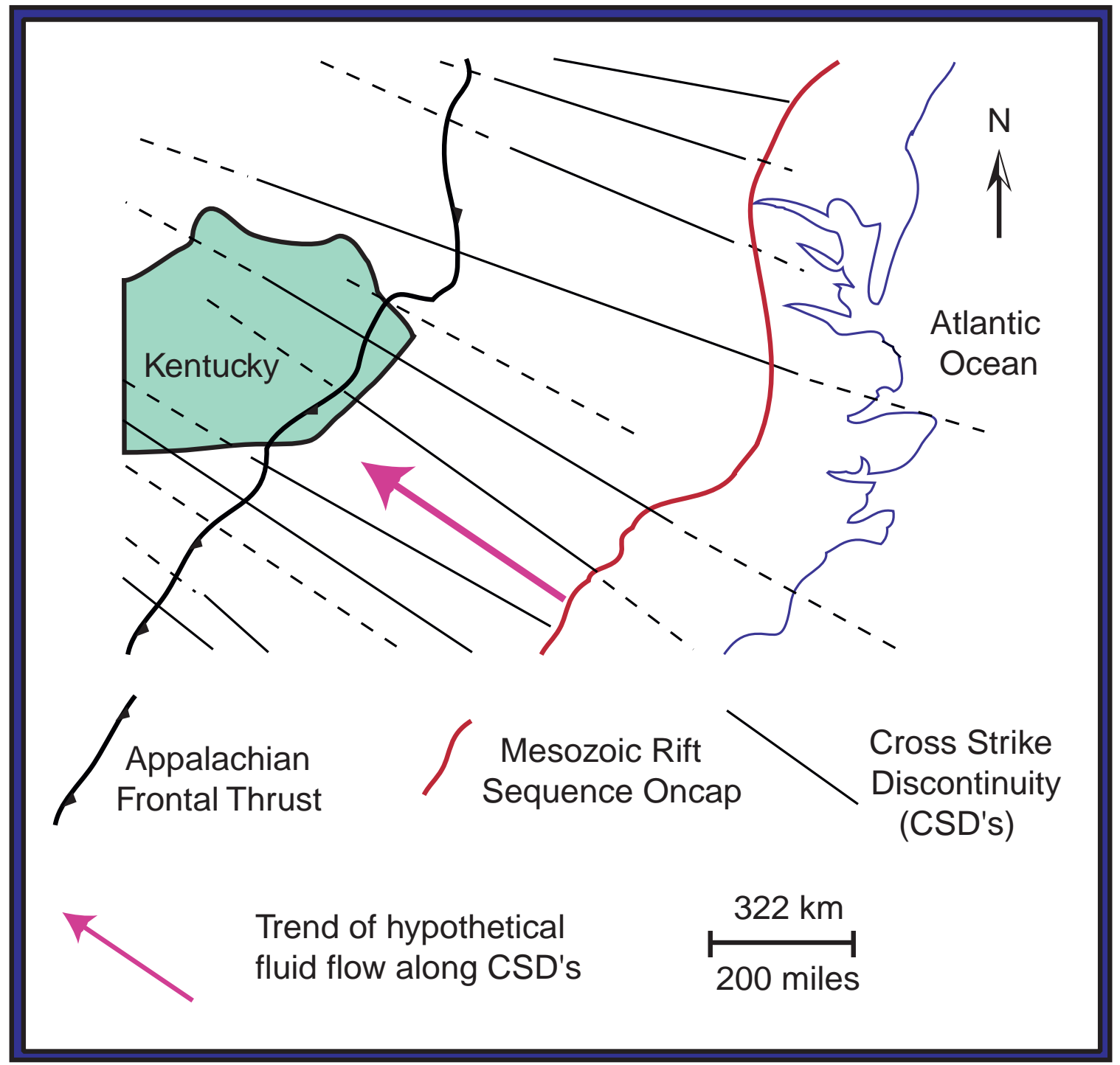

Figure 5. CSD's of the Eastern United States. Eastern Kentucky shown in green (Coleman and others, 1988). 


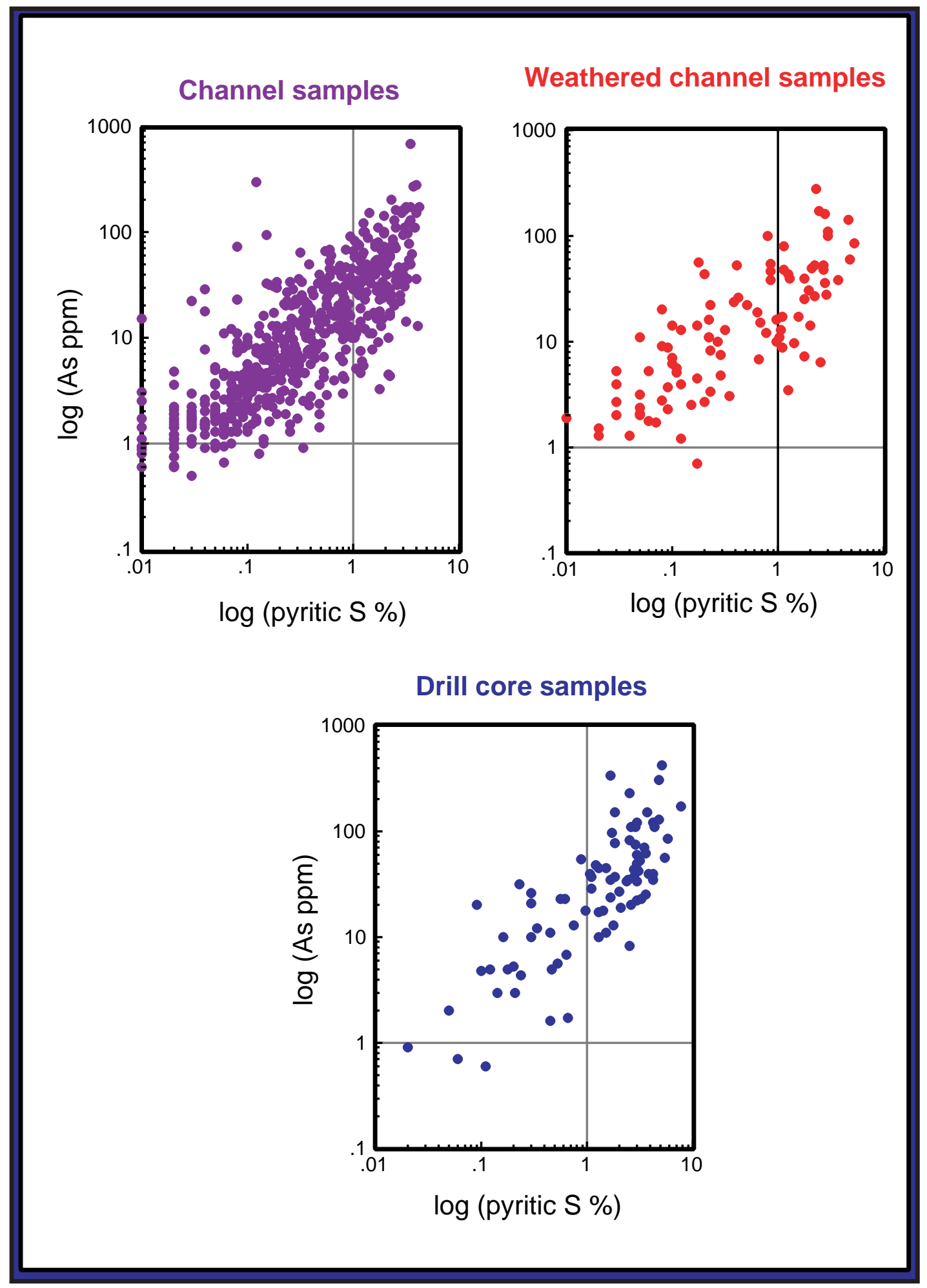

Figure 6. Cross plots of Arsenic (ppm) versus pyritic sulfur (wt \% S) in drill-core, channel, and weathered channel coal samples. 
Diagenetic pyrite formed early in the peat's history would not accumulate arsenic unless arsenic was in the peat pore water. Whether the peat was influenced by seawater or not, it is unlikely that large amounts of arsenic would be available during, or shortly after, peat deposition. Most arsenic in the Warrior Basin to the South is associated with epigenetic pyrite formed when mineralizing fluids moved through the Alabama coals after deposition and early diagenesis (see Goldhaber and others, submitted). We hypothesize that the enrichment of arsenic in eastern Kentucky coals occurred under similar conditions to those in Alabama.

\section{ARSENIC IN NURE SAMPLES FROM THE KENTUCKY APPALACHIAN BASIN}

Twelve hundred forty NURE (National Uranium Resource Evaluation) stream sediment samples from the Kentucky Appalachian Basin were reanalyzed because of improved detection limits for arsenic and other metals. Ninety-three per cent of the samples have arsenic concentrations less than $10 \mathrm{ppm}$, the average arsenic abundance in shale (Wedepohl, 1974) (fig. 7). High arsenic concentrations along the western edge of the basin correlate with exposure of arsenic-enriched Upper Devonian black shale (Leventhal and Kepferle, 1982) (fig. 8).

Some stream sediments from the eastern Kentucky coal region are high in arsenic (Pennsylvanian rocks in fig. 8). Arsenic-enrichment in this portion of the basin likely is associated with streams impacted by coal mining and agriculture (Porter and others; 1995).

\section{Possible Controls on Arsenic in NURE Samples from the Kentucky Appalachian Basin}

Elevated amounts of iron oxyhydroxide precipitates in streams affected by coal mining have been reported in eastern Kentucky coal region (Porter and others, 1995). Arsenic released to the environment either through natural weathering processes or human activity such as coal mining is adsorbed onto iron oxyhydroxide precipitates (Cullen and Reimer, 1989).

The cross plot in Figure 9 shows the relation between arsenic concentration (ppm) and iron concentration (wt\%) in NURE stream sediment samples. In the coal region (Kentucky Appalachian Basin proper), there is a weak, but observable positive relation between the two variables. The scatter in these data is likely due to variability in the availability of arsenic in the drainage basin. On the northwest margin of the basin where the Devonian black shale crops out, there is a strong positive relation between the two variables. Several samples from the coal region (pink dots) plot in the Devonian black shale field and suggest that these stream sediments accumulated arsenic over and above most stream sediment in the coal region. 


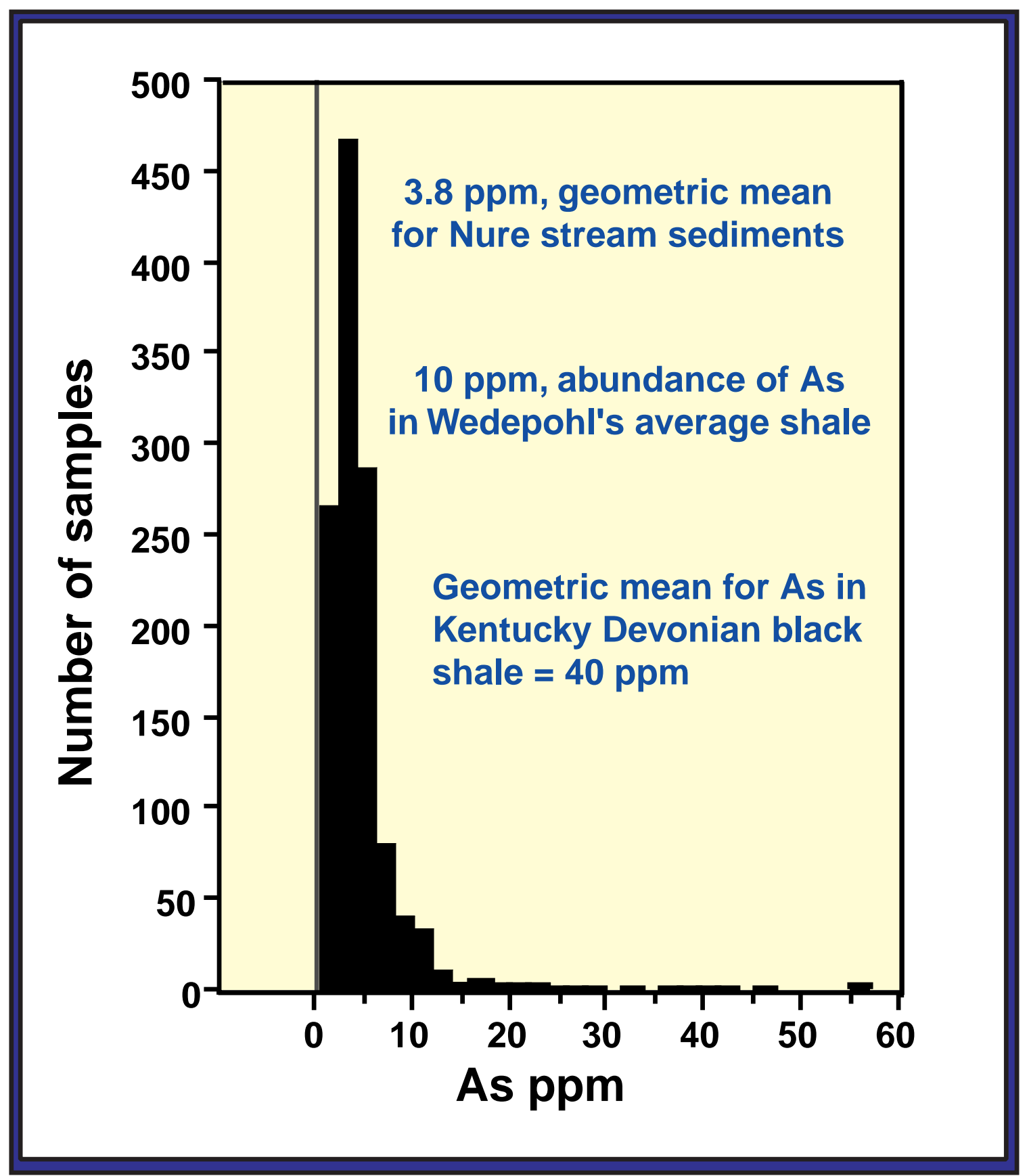

Figure 7. Histogram of arsenic concentrations in 1,200 NURE stream sediments, Kentucky Appalachian Basin (abundance data from Wedepohl, 1974; Kentucky Devonian shale data from 14 core samples of Leventhal and Kepferle, 1982). 


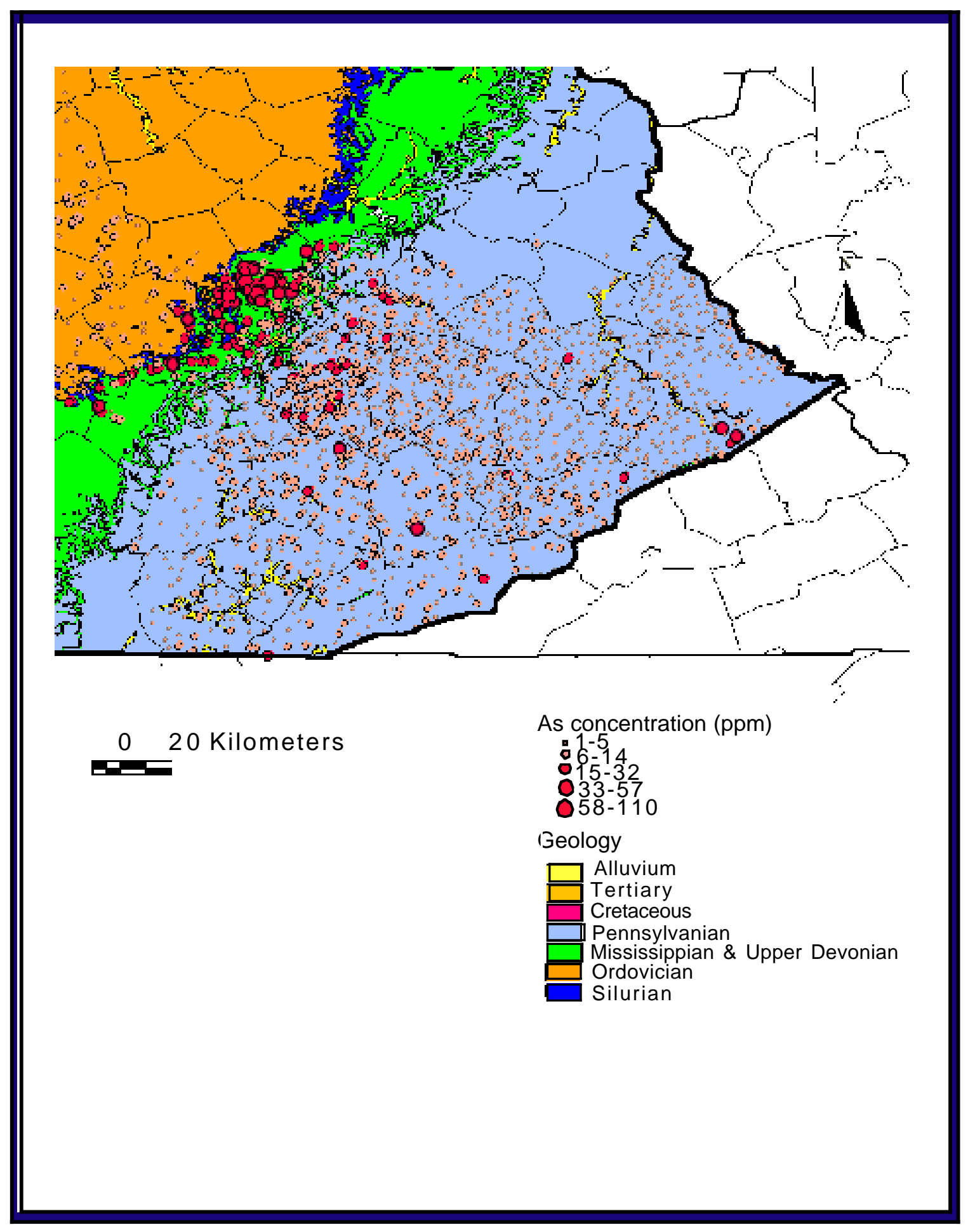

Figure 8. Map showing generalized geology of the Kentucky Appalachian Basin, and arsenic concentrations (ppm) in NURE stream sediments. 


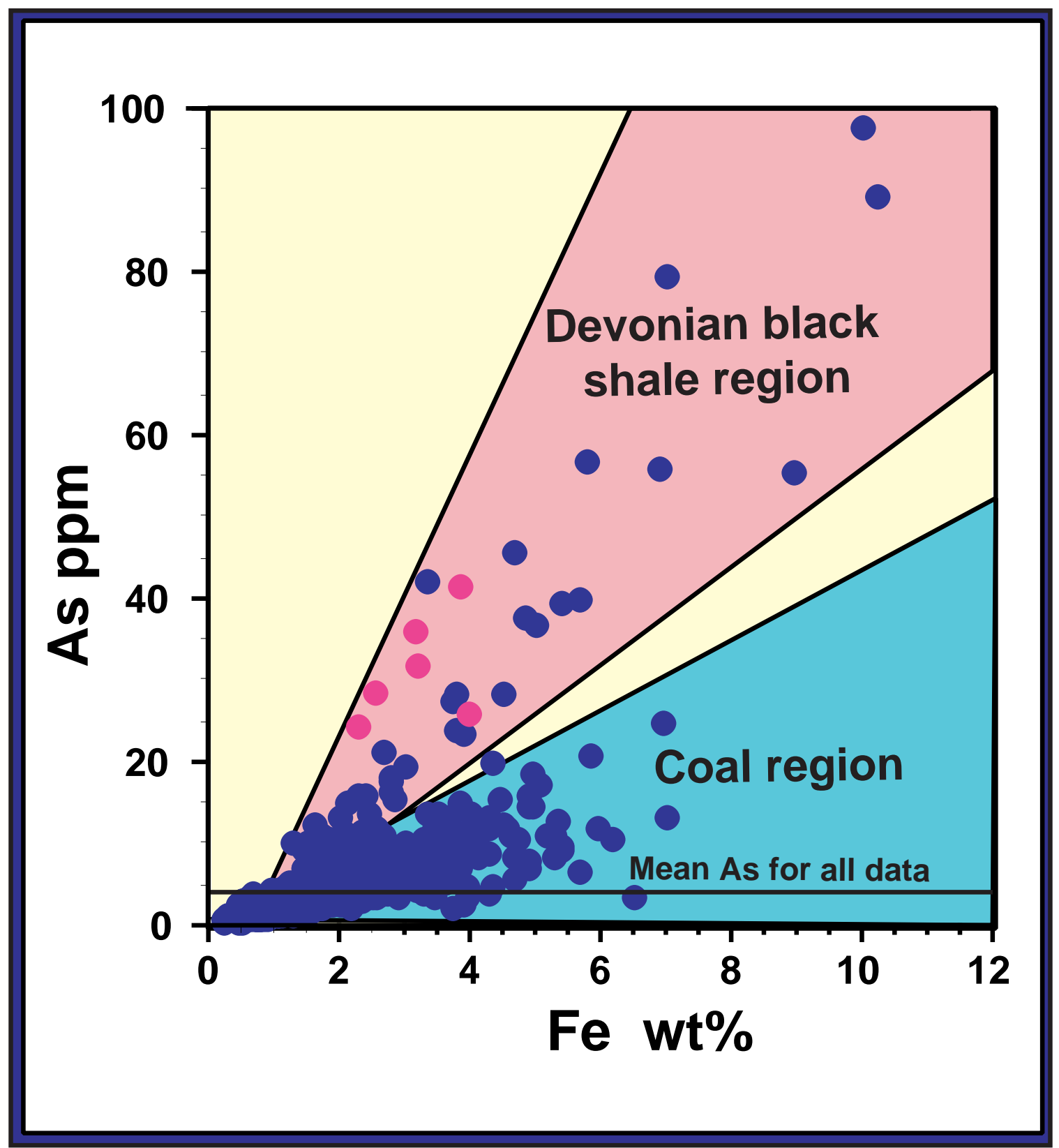

Figure 9. Cross plot of arsenic (ppm) versus iron (wt \%) divided into regions within the basin. Geometric mean calculated from all data reanalyzed in this study. Pink dots; data from the Coal region that plot with those from the Devonian black shale region.

\section{ARSENIC IN SHALE AND COAL COLLECTED IN THE KENTUCKY APPALACHIAN BASIN}

Our sampling of the Devonian New Albany Formation was designed to test the working hypotheses that the formation is the source of anomalously high concentrations of Arsenic in sediments on the western margin of the Kentucky Appalachian basin. In 
addition, our study will investigate the weathering processes that transport the arsenic to the stream sediments. Five types of New Albany Shale samples were collected at an outcrop near Clay City (fig. 10A). The first was surface samples from a horizontal transect across the face of a roadcut (fig. 10A and 10B). These samples $(n=17)$ represent a weathering profile related to topography in the region and surface weathering since the roadcut. The second type was unexposed samples directly behind the first type (fig. 10B). These samples $(n=9)$ represent a weathering profile related to topography, with minimal surface weathering related to the road cut. The third type was soils that developed on the side of the transect $(n=4)$ (fig. 10A). The fourth type was from a drill core taken a short distance from the outcrop. These samples $(n=22)$ represent unweathered shale material. In addition, shale fragments that had been mechanically weathered from the outcrop $(n=3)$ and salt crusts on the shale $(n=4)$ were also sampled. The semiquantitative XRF data for all samples are in Appendix II.

Coal samples were collected at 4 mines to test the hypothesis that arsenic mobilized during weathering of pyrite in the Kentucky coal is the source of arsenic anomalies in the Appalachian basin proper. Bench samples were collected at each mine for analyses by the USGS coal package (data not included in this report). Pyrite and associated coal ( $\mathrm{n}=$ 11) were sampled at each mine except at one where no pyrite could be found. The semiquantitative XRF data on these pyritic samples are in Appendix II. Three of the 11 coals are from the fold-belt at Pound Gap (Chestnut, 1998) (fig. 10C) and were collected to test the hypothesis of large-scale fluid flow through this region.

\section{Relation between Iron and Arsenic in the Eastern Kentucky Coal}

Compared to the coal in the Warrior Basin in Southern Appalachia, there is visually less pyrite in the Kentucky coal. Also, there appears to be much less structural features in the coal suggesting fewer conduits for epigenetic fluid flow. Figure 11 indicates that there is an increase in arsenic concentration with increasing iron content in Eastern Kentucky coals. This is consistent with the data in Figure 4 from the USGS coal database, as most of the iron in these mineral-poor coals is assumed to be pyrite, although some siderite nodules have been noted. The coal from the Pine Mountain Thrust contained no visible pyrite, but one sample contains appreciable arsenic (74 ppm As)—a possible imprint left by migrating fluids through the region. 


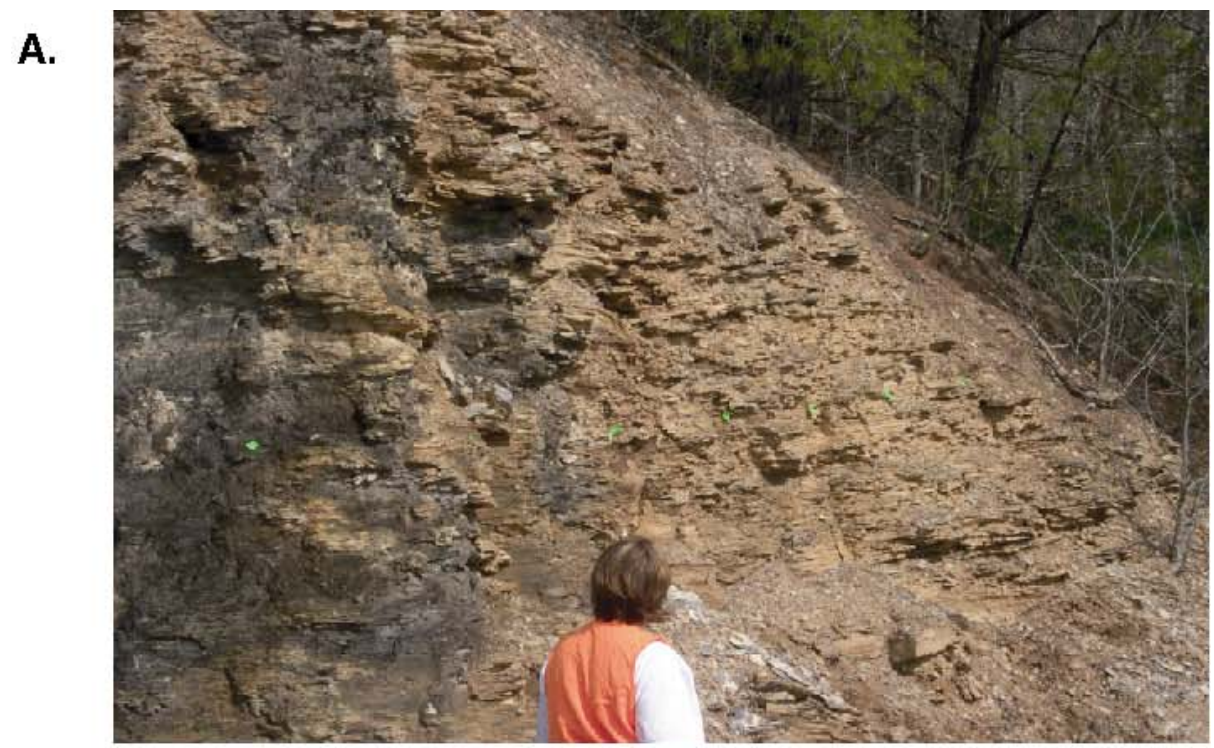

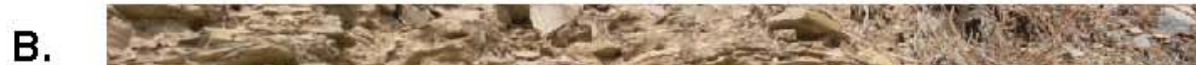

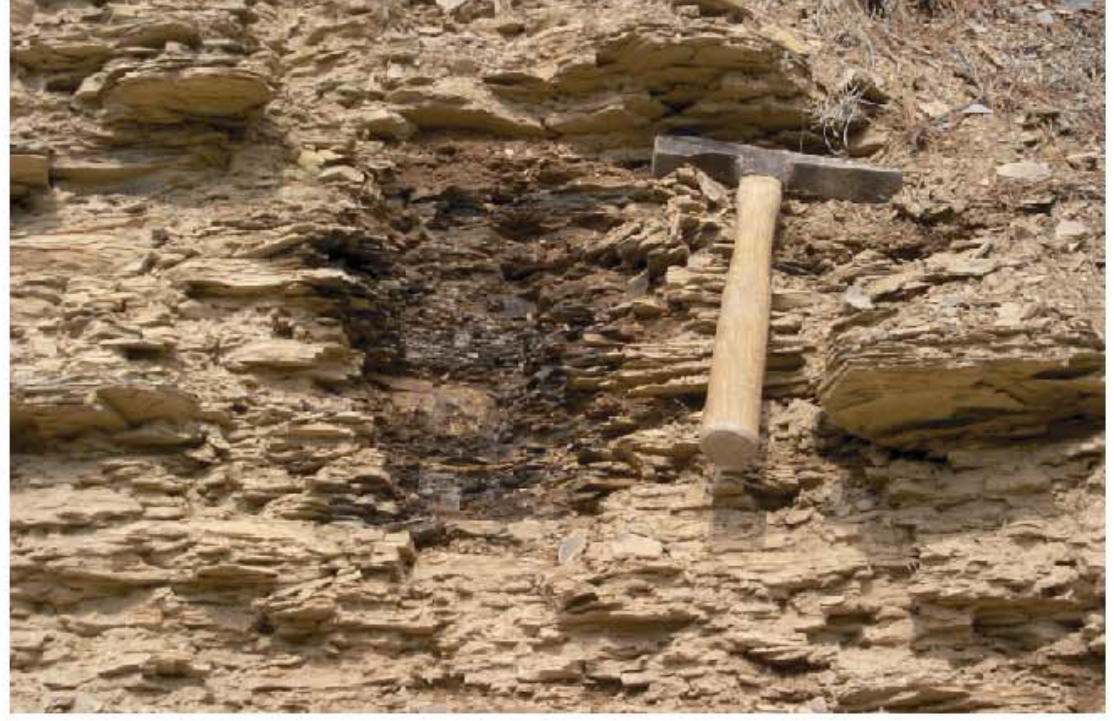

c.

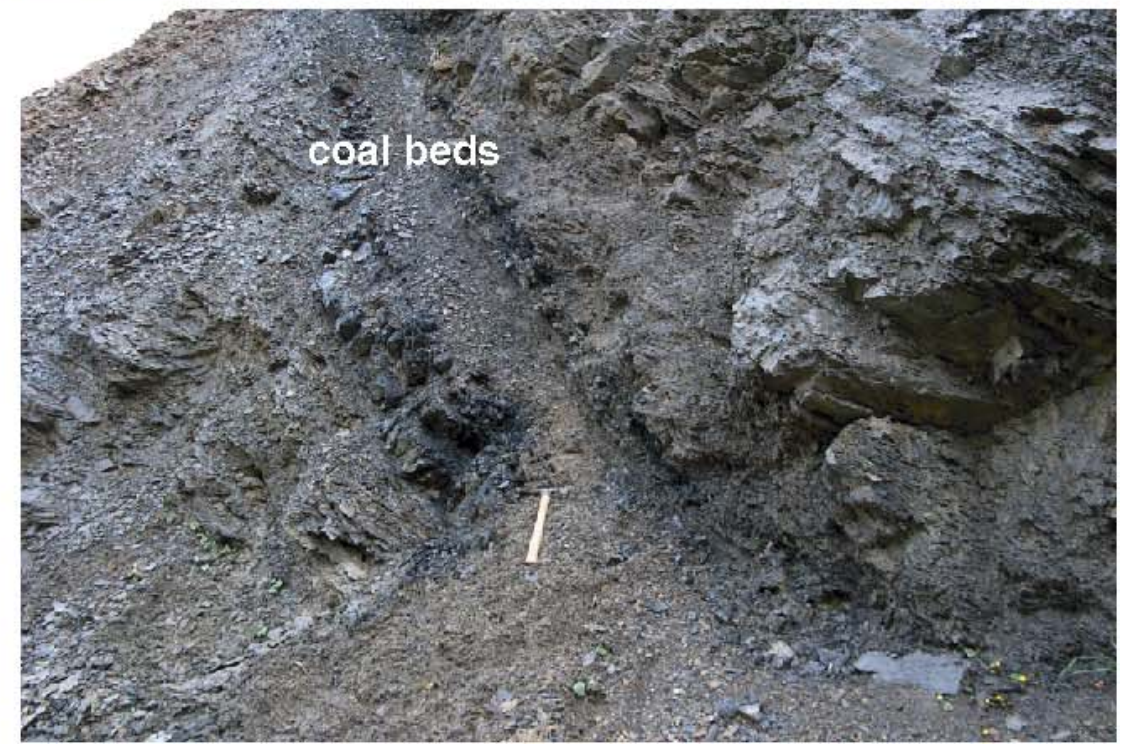


Figure 10. Photos of A) transect across the Devonian outcrop (Clay City, Kentucky), B) surface weathered and "fresh" shale behind, and C) two coal beds in Pound Gap roadcut.

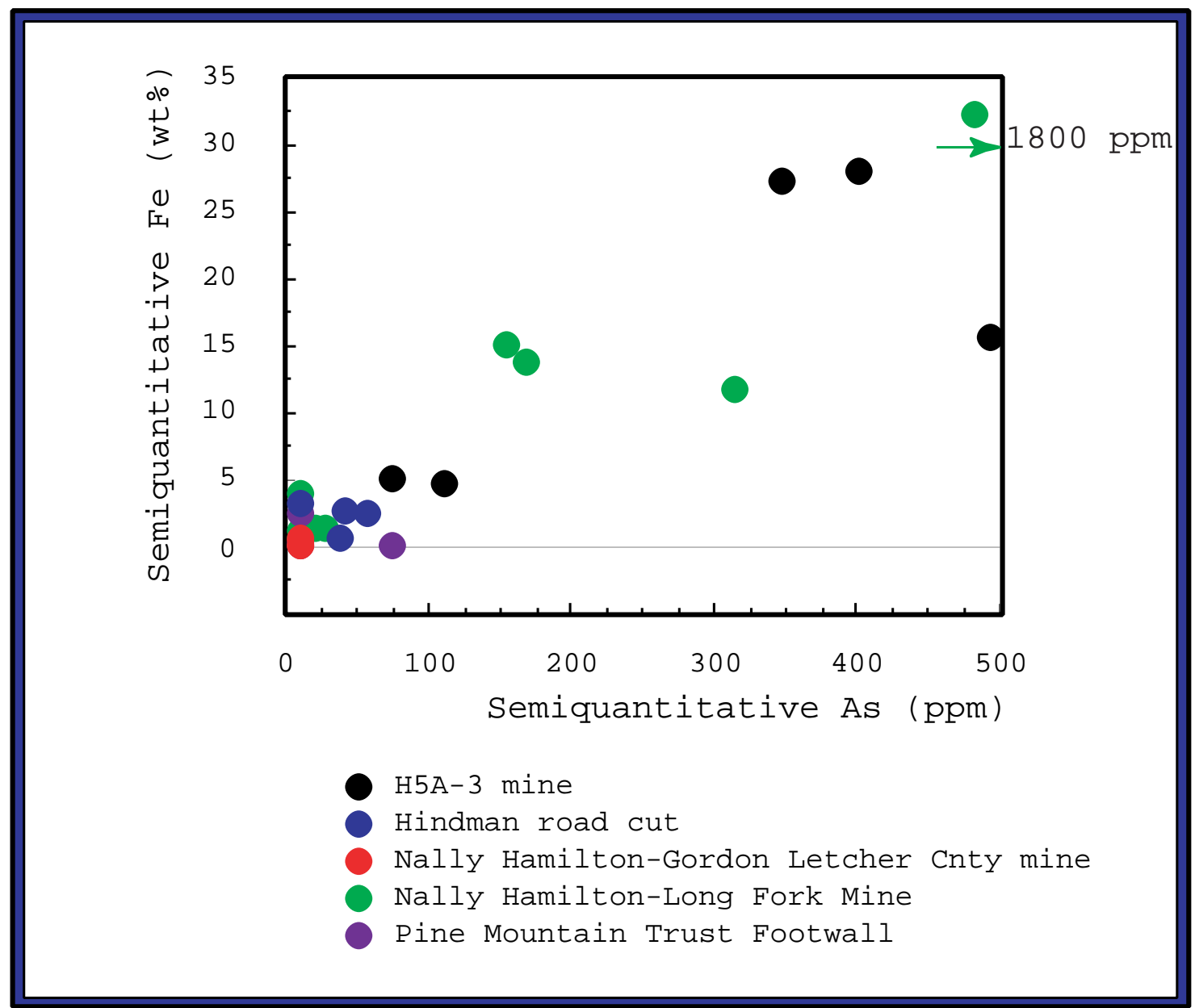

Figure 11. Cross plot of iron versus arsenic in coal collected from the Kentucky Appalachian basin.

Relation between Iron and Arsenic in the Devonian Shale

There appears to be a strong relation between iron (likely as iron hydroxides) on weathered shale samples from the outcrop and arsenic content (fig. 12A). This relation suggests that arsenic and iron from pyrite in the shale is mobilized during weathering and redeposited as iron hydroxides on the shale surfaces. Our preliminary working hypothesis mechanically transports the arsenic into stream sediments bound to this weathering rind. Once in the stream, the arsenic will remain bound to the iron hydroxides until chemical conditions radically change--the iron hydroxide dissolves or the $\mathrm{pH}$ of the stream increases dramatically causing the arsenic to desorb from the iron hydroxide surface. 
Salts (mostly iron sulfates) forming on the outcrop accumulate arsenic (concentrations up to $170 \mathrm{ppm} \mathrm{As}$; Tuttle and others, 2001). When these salts dissolve, they release the arsenic. This arsenic probably ends up in the iron oxyhydroxides that either form on the shale fragments or on the stream sediments (Tuttle and others, 2001).

In the core, both the shale samples and those containing significant pyrite as nodules, beds, etc. show a similar linear relationship between Fe and As (fig. 12B); however, the slope of the linear best fit is different between the two core populations. This difference indicates that the pyrite-enriched samples have a lesser amount of arsenic per unit iron than the shale. The residence of arsenic in the shale samples may be different than pyrite, or arsenic may have been less available for incorporation into the more massive pyrite that formed during later stages of diagenesis.

\section{CONCLUDING REMARKS}

Eastern Kentucky coal has less pyrite than that in the Warrior basin to the south. The difference may reflect less structural deformation in the Kentucky coal during the Allegheny Orogeny, hence fewer fluid migration pathways. Most of the epigenetic pyrite we sampled in the Kentucky coal was arsenic enriched. Weathering of this pyrite on mine-waste piles or on outcrop, will produce acid solutions that mobilize the iron and arsenic. Long-range transport of arsenic is unlikely because it will adsorb onto iron oxyhydroxides as the acidic water is neutralized.

Along the western margin of the Kentucky Appalachian basin, the source of arsenic in the stream sediment appears to be the New Albany Shale. Weathering rinds on the shale surfaces are enriched in arsenic relative to the unweathered shale surfaces. It appears that weathering of arsenic-rich pyrite within the shale mobilizes arsenic and iron that is redeposted on the shale surface. Mechanical transport of weathering rinds on shale fragments appear to be the primary transport mechanism of the arsenic into the stream sediment. Some arsenic accumulates in weathering salts on the outcrop. Arsenic in these salts is mobilized during salt dissolution, but likely is adsorbed quickly onto iron oxides.

Currently, quantitative chemical and stable isotopic data are being collected. Data will be used to test our working hypotheses developed for arsenic, determine source, residence and mobility of other trace elements in Kentucky coal and Devonian New Albany Shale, and pick additional sites for more detailed follow-up studies on trace-metal effects on streams and ecosystems. 


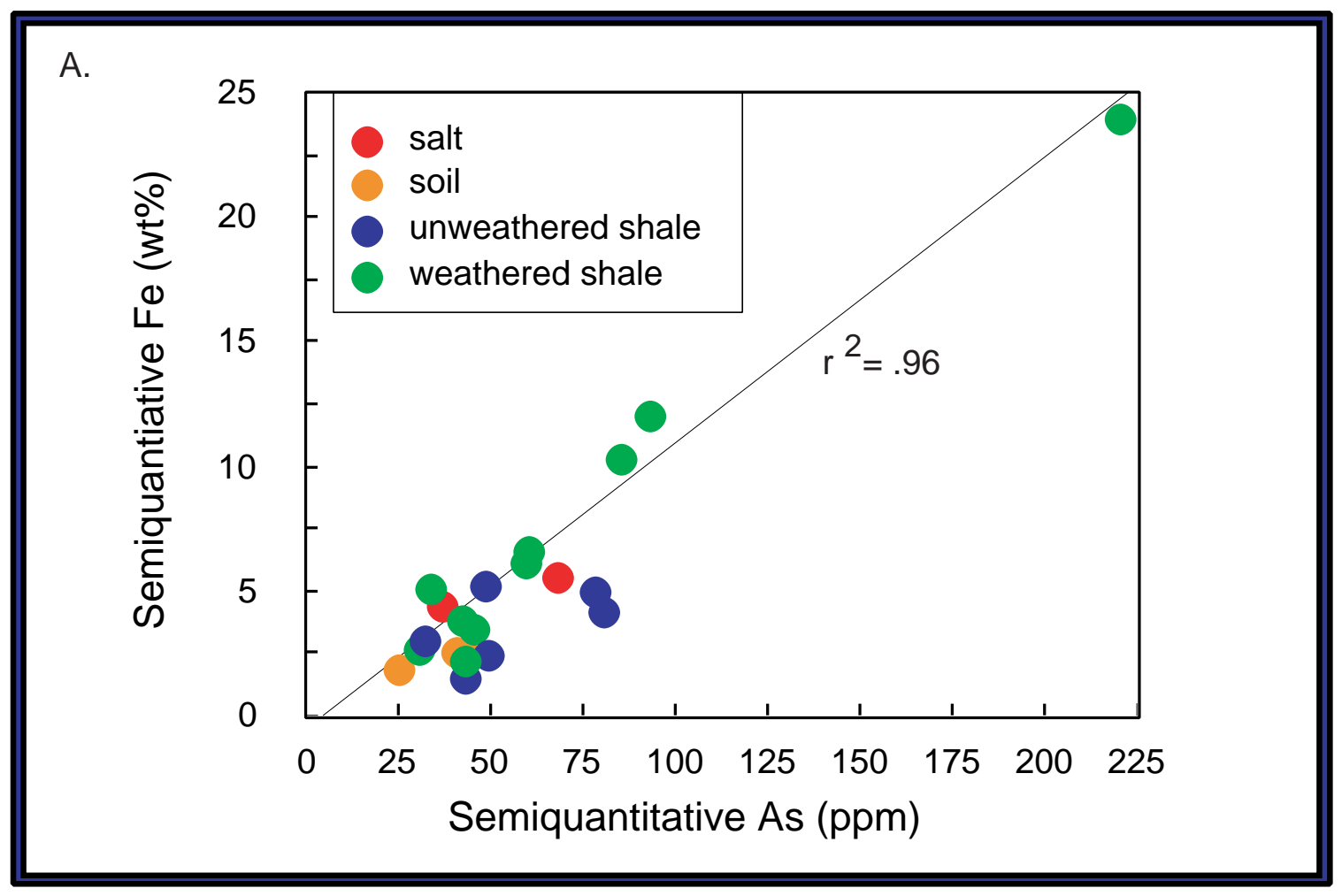

B.

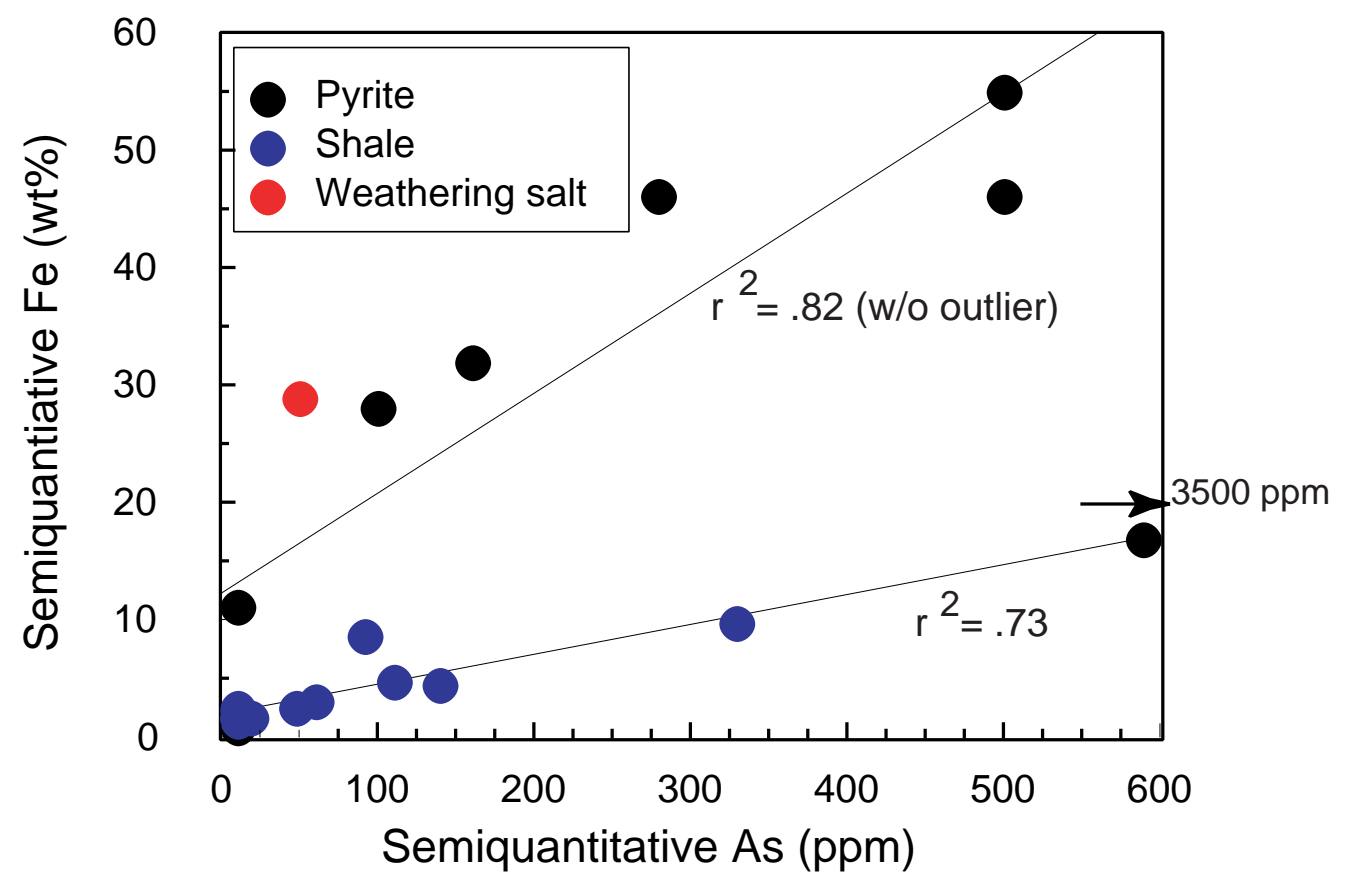

Figure 12. Cross plots of A) iron versus arsenic in outcrop samples (regression on weathered samples only) and B) iron versus arsenic in core samples of shale and shale containing large amounts of pyrite; outlier not included in 


\section{REFERENCES}

Bragg, L.J., Oman, J.K., Tewalt, S.J., Oman, C.L., Rega, N.H., Washington, P.M., and Finkelman, R.B., 1997, U.S. Geological Survey Coal Quality (COALQUAL) Database: Version 2.0: U.S. Geological Survey Open-file Report 97-134, CDROM.

Britton, L.J., Anderson, C.L., Goolsby, D.A., and Van Haveren B.P., 1989, Summary of the U.S. Geological Survey and U.S. Bureau of Land Management National Coal-Hydrology Program, 1974-84: U.S. Geological Survey Professional Paper 1464, 183 p.

Chesnut, D.R., Jr., 1998, Geology of the Pound Gap Road cut, Letcher County, Kentucky: 1998 Annual Field Conference of the Kentucky Society of Professional Geologists, $169 \mathrm{p}$.

Coleman, J.L., Groshong, R.H., Rheams, K.F., Neathery, T.L., and Rheams, L.J., 1988, Structure of the Wills Valley Anticline-Lookout Mountain Syncline Between the Rising Fawn and Anniston CSD's, Northeast Alabama, a guidebook for the 25th annual field trip of The Alabama Geological Society, 1988, 120 p.

Coleman, S.L., and Bragg, L.J., 1990, Distribution and mode of occurrence of arsenic in coal, in, Chyi, L.L. and Chou, C.L., eds., Recent advances in coal geochemistry: Geological Society Special Paper 248, p. 13-26.

Cullen, W.R., and Reimer, K.J., 1989, Arsenic speciation in the environment: Chemical Reviews, v. 89, no. 4, p. 713-764.

Goldhaber, M.B., Lee, R.C., Hatch, J.R., Pashin, J.C., and Treworgy, J., in press, Role of large scale fluid-flow in subsurface arsenic enrichment., in, Welch, A. and Stollenwerk, K., (eds.), Arsenic in Ground Water: Occurrence and Geochemistry. Norwell, Kluwer Academic Puplishers.

Hower, J.C., and Rimmer, S.M., 1991, Coal rank trends in the Central Appalachian coalfield: Virginia, West Virginia, and Kentucky: Organic Geochemistry, v. 17, p. 161-173.

Leventhal, J.S., and Kepferle, R.C., 1982, Geochemistry and geology of strategic metals and uranium in Devonian shales of the Eastern Interior United States: Synthetic Fuels from Oil Shales II. Proceeding of the symposium sponsored by Institute of Gas Technology, Nashville, Tennessee, October 26-29, 1982.

Matthews, R.D., Janka, J.C., Dennison, J.M., 1981, Extraction methods and reserves of Devonian-Mississippian oil shales in Kentucky, in, Pryor, W.A., Maynard J.B., Potter, P.E., Fist, H.N., Kepferle, R.C., Kiefer, J., eds., Energy Resources of Devonian-Mississippian shales of Eastern Kentucky: Annual Field Conference of the Geological Society of Kentucky, April 9-11, 1981, p. 8.

Porter, S.D., White, K.D., and Clark, J.R., 1995, Water-quality assessment of the Kentucky River Basin, Kentucky: Distribution of metals and other trace elements in sediment and water, 1987-90: U.S. Geological Survey Water Resources Investigations Report 94-4134, 184 p.

Tuttle, M.L.W., Goldhaber, M.B., and Breit, G.N., 2001, Mobility of metals from weathered black shale: the role of salt efflorescences: Geological Society of America Abstracts with Programs, v. 33, p. A-191.

Wedepohl, K.H., 1974, Handbook of Geochemistry. Berlin, Springer-Verlag. 


\section{APPENDIX I--NURE STREAM SEDIMENT DATA (REANALYZED)}

The $<100 \mu \mathrm{m}$ fraction of the sample was generally analyzed. In some cases, the $<40 \mu \mathrm{m}$ and $>100 \mu \mathrm{m}$ fraction was analyzed if the $<100 \mu \mathrm{m}$ fraction was not available. Both fractions from 43 samples were analyzed to cross check concentrations. The lowest arsenic concentration of the pair was on the average $30 \%$ lower than the higher concentration. In $77 \%$ of the pairs, the higher concentration was in the finer-grained fraction.

\section{Analytical Methods}

Arsenic was determined by weighing $0.1 \mathrm{~g}$ of sample into a zirconium crucible. Approximately $0.75 \mathrm{~g}$ of sodium peroxide is added and mixed. The mixture is heated in a muffle furnace set at $750^{\circ} \mathrm{C}$ for four minutes. The sample is cooled then $15 \mathrm{ml}$ of water and $5 \mathrm{ml}$ of concentrated $\mathrm{HCl}$ is added. The mixture is shaken and $0.25 \mathrm{ml}$ of an ascorbic acid $\mathrm{KI}$ solution is added then diluted with $20 \% \mathrm{HCl}$ and let to stand overnight. Arsenic is then measured using hydride generation atomic absorption spectrometry. The optimum concentration ranges without sample dilution for arsenic in various solid phase sample media is $0.6 \mathrm{ppm}$ to $20 \mathrm{ppm}$. Sample weight is $0.1 \mathrm{~g}$ and data will be deemed acceptable if recovery of As is $\pm 20 \%$ at five times the LOD and the calculated percent RSD of duplicate samples is no greater than $20 \%$.

Forty major, minor, and trace elements were determined in geological materials by inductively coupled plasma-atomic emission spectrometry (ICP-AES). The sample is decomposed using a mixture of hydrochloric, nitric, perchloric, and hydrofluoric acids at low temperature. The digested samples are aspirated into the ICP-AES discharge where the elemental emission signal is measured simultaneously for the forty elements. Calibration is performed by standardizing with digested rock reference materials and a series of multi-element solution standards. Sample weight was $0.2 \mathrm{~g}$

Reporting limits for 40 elements by ICP-AES:

$\begin{array}{lc}\text { Element: } & \text { Concentration Range } \\ \text { Aluminum, Al } & 0.005-50 \% \\ \text { Calcium, Ca } & 0.005-50 \% \\ \text { Iron, Fe } & 0.02-25 \% \\ \text { Potassium, K } & 0.01-50 \% \\ \text { Magnesium, Mg } & 0.005-5 \% \\ \text { Sodium, Na } & 0.005-50 \% \\ \text { Phosphorous, P } & 0.005-50 \% \\ \text { Titanium, Ti } & 0.005-25 \% \\ \text { Silver, Ag } & 2-10,000 \mathrm{ppm} \\ \text { Arsenic, As } & 10-50,000 \mathrm{ppm} \\ \text { Gold, Au } & 8-50,000 \mathrm{ppm} \\ \text { Barium, Ba } & 1-35,000 \mathrm{ppm} \\ \text { Beryllium, Be } & 1-5,000 \mathrm{ppm}\end{array}$


Element:

Bismuth, Bi

Cadmium, $\mathrm{Cd}$

Cerium, $\mathrm{Ce}$

Cobalt, $\mathrm{Co}$

Chromium, $\mathrm{Cr}$

Copper, $\mathrm{Cu}$

Europium, Eu

Gallium, Ga

Holmium, Ho

Lanthanum, La

Lithium, Li

Manganese, Mn

Molybdenum, Mo

Niobium, $\mathrm{Nb}$

Neodymium, Nd

Nickel, Ni

Lead, $\mathrm{Pb}$

Scandium, Sc

Tin, Sn

Strontium, $\mathrm{Sr}$

Tantalum, Ta

Thorium, Th

Uranium, U

Vanadium, V

Yttrium, $\mathrm{Y}$

Ytterbium, Yb

Zinc, Zn

\section{Concentration Range}

10 - 50,000 ppm

2 - 25,000 ppm

5 - 50,000 ppm

2 - 25,000 ppm

2 - 25,000 ppm

2 - 15,000 ppm

2 - 5,000 ppm

4 - 50,000 ppm

$4-5,000$ ppm

2 - 50,000 ppm

2 - 50,000 ppm

4 - 50,000 ppm

2 - 50,000 ppm

4 - 50,000 ppm

9 - 50,000 ppm

3 - 50,000 ppm

4 - 50,000 ppm

2 - 50,000 ppm

5 - 50,000 ppm

2 - 15,000 ppm

$40-50,000$ ppm

6 - 50,000 ppm

$100-100,000 \mathrm{ppm}$

2 - 30,000 ppm

2 - 25,000 ppm

1 - 5,000 ppm

2 - 15,000 ppm

Data is deemed acceptable if recovery for all 40 elements is $\pm 15 \%$ at five times the Lower Limit of Determination (LOD) and the calculated Relative Standard Deviation (RSD) of duplicate samples is no greater than $15 \%$. 


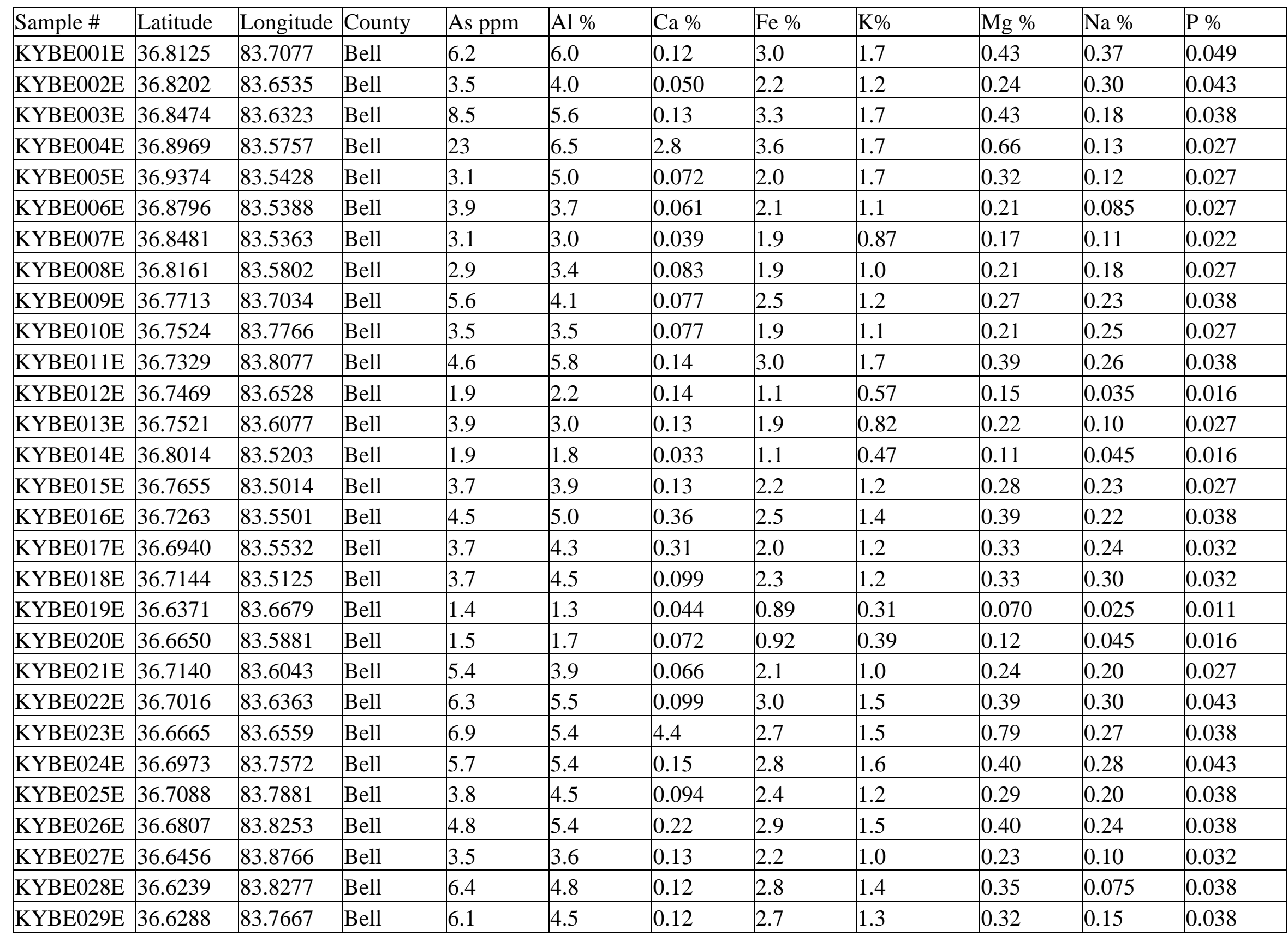




\begin{tabular}{|c|c|c|c|c|c|c|c|c|c|c|c|}
\hline KYBE031E & 36.6184 & 83.9327 & Bell & 7.3 & 5.0 & 0.28 & 2.9 & 1.4 & 0.36 & 0.25 & 0.043 \\
\hline KYBH002 & 37.6802 & 83.4012 & Breathitt & 3.6 & 3.6 & 0.050 & 1.9 & 1.1 & 0.20 & 0.44 & 0.027 \\
\hline KYBH003 & 37.6728 & 83.3583 & Breathitt & 3.0 & 3.6 & 0.061 & 1.6 & 1.2 & 0.20 & 0.47 & 0.022 \\
\hline KYBH006 & 37.6533 & 83.3745 & Breathitt & 3.7 & 4.5 & 0.083 & 2.3 & 1.3 & 0.31 & 0.36 & 0.032 \\
\hline KYBH007 & 37.6625 & 83.3440 & Breathitt & 3.4 & 3.5 & 0.061 & 1.9 & 1.1 & 0.23 & 0.28 & 0.027 \\
\hline KYBH008 & 37.6330 & 83.2879 & Breathitt & 1.2 & 2.3 & 0.039 & 1.1 & 0.92 & 0.14 & 0.24 & 0.016 \\
\hline KYВH009 & 37.6536 & 83.3127 & Breathitt & 3.1 & 3.5 & 0.050 & 1.9 & 1.1 & 0.23 & 0.39 & 0.022 \\
\hline KYBH010 & 37.6690 & 83.3075 & Breathitt & 2.4 & 2.4 & 0.044 & 1.5 & 0.82 & 0.15 & 0.19 & 0.022 \\
\hline KYBH014 & 37.6340 & 83.2254 & Breathitt & 1.5 & 1.4 & 0.018 & 0.83 & 0.52 & 0.069 & 0.11 & 0.010 \\
\hline КYBH015 & 37.6041 & 83.2272 & Breathitt & 2.0 & 2.7 & 0.053 & 1.1 & 0.98 & 0.15 & 0.22 & 0.015 \\
\hline KYВH016 & 37.6182 & 83.2078 & Breathitt & 2.5 & 3.4 & 0.053 & 1.5 & 1.1 & 0.18 & 0.19 & 0.015 \\
\hline КYВН017 & 37.5781 & 83.1960 & Breathitt & 2.1 & 3.0 & 0.047 & 1.3 & 1.1 & 0.16 & 0.20 & 0.020 \\
\hline KYBH018 & 37.5508 & 83.3414 & Breathitt & 2.8 & 3.1 & 0.053 & 1.5 & 0.99 & 0.18 & 0.41 & 0.020 \\
\hline КҮВН019 & 37.5788 & 83.3173 & Breathitt & 4.3 & 9.0 & 2.5 & 3.2 & 2.0 & 0.73 & 0.23 & 0.035 \\
\hline КYBН020 & 37.5936 & 83.2831 & Breathitt & 1.8 & 2.3 & 0.035 & 1.0 & 0.86 & 0.11 & 0.19 & 0.010 \\
\hline KYBH021 & 37.5732 & 83.2550 & Breathitt & 1.5 & 2.2 & 0.61 & 0.82 & 0.9 & 0.12 & 0.18 & 0.015 \\
\hline KYBH022 & 37.6076 & 83.1622 & Breathitt & 1.5 & 2.7 & 0.047 & 1.1 & 0.97 & 0.14 & 0.17 & 0.010 \\
\hline KYBH023 & 37.6294 & 83.1789 & Breathitt & 1.6 & 3.3 & 0.047 & 1.3 & 1.1 & 0.18 & 0.19 & 0.020 \\
\hline
\end{tabular}




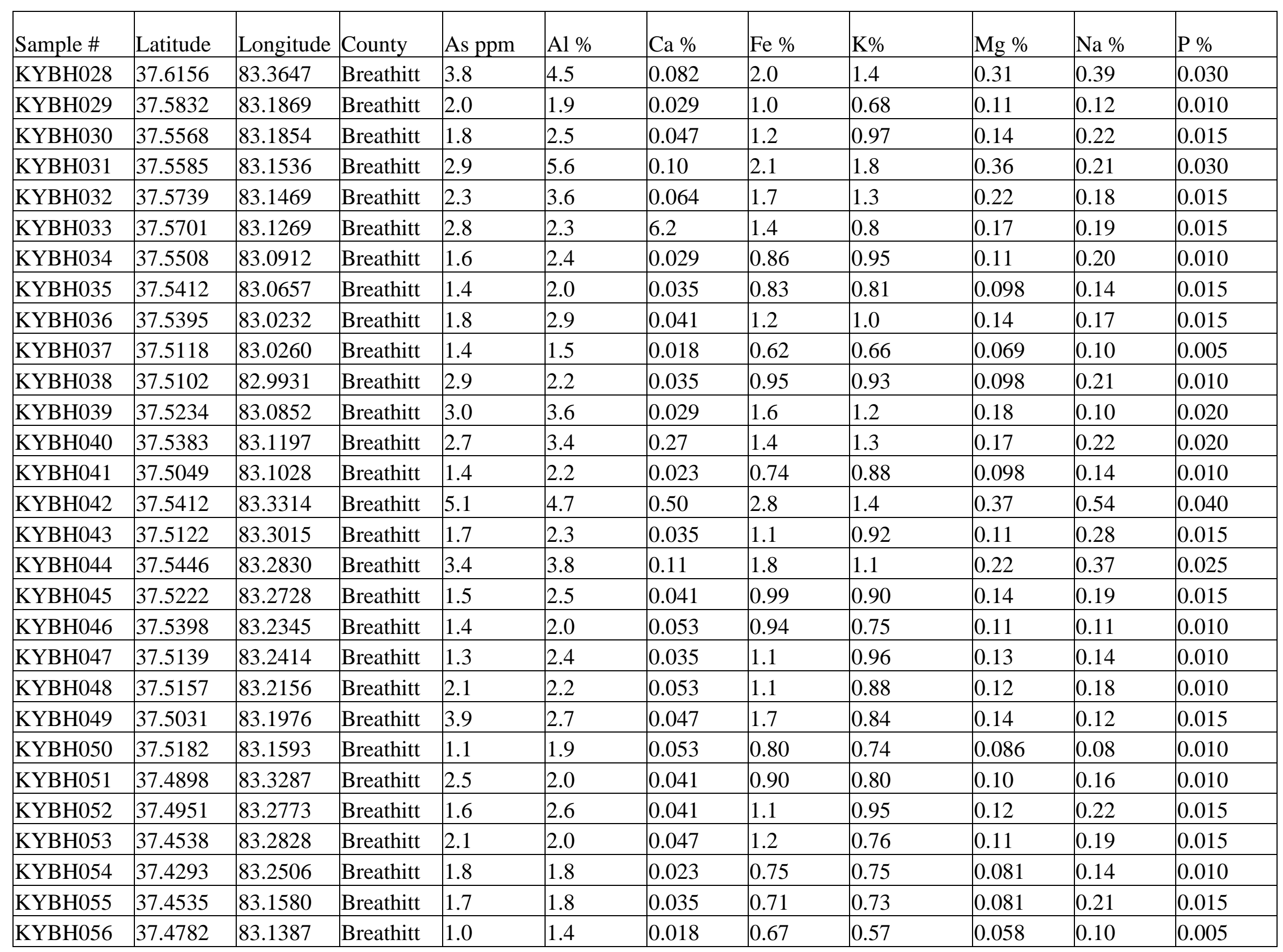




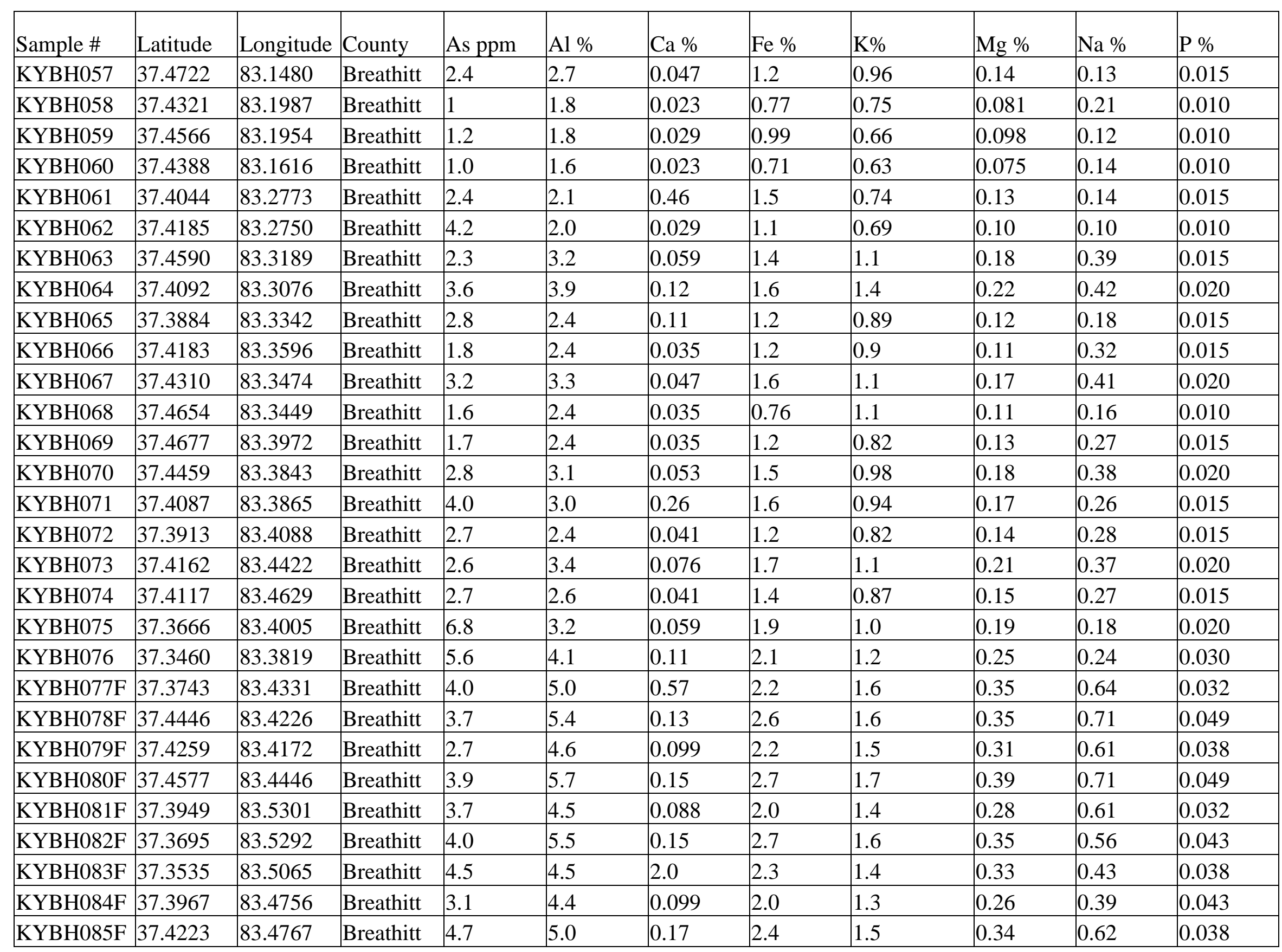




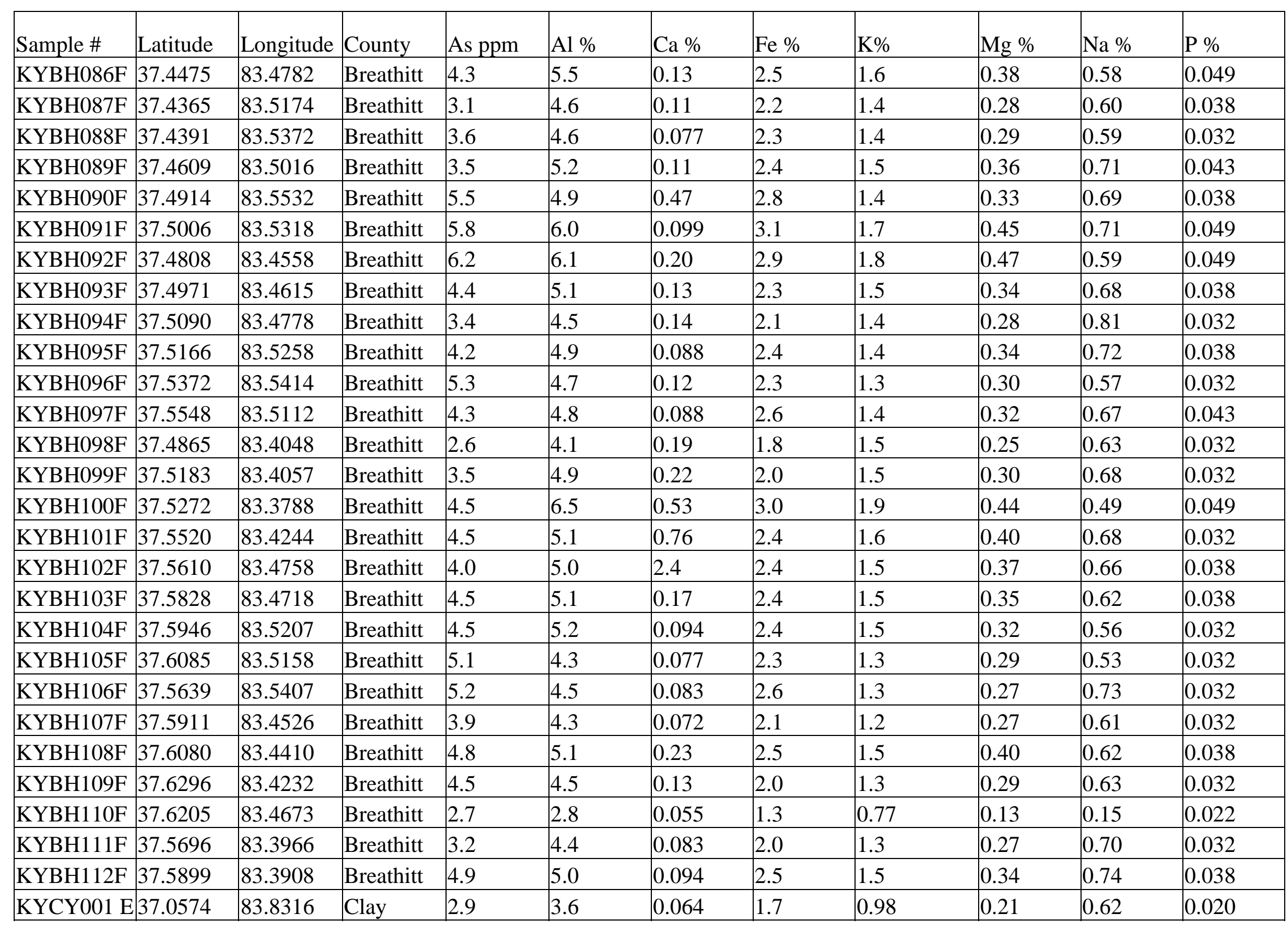




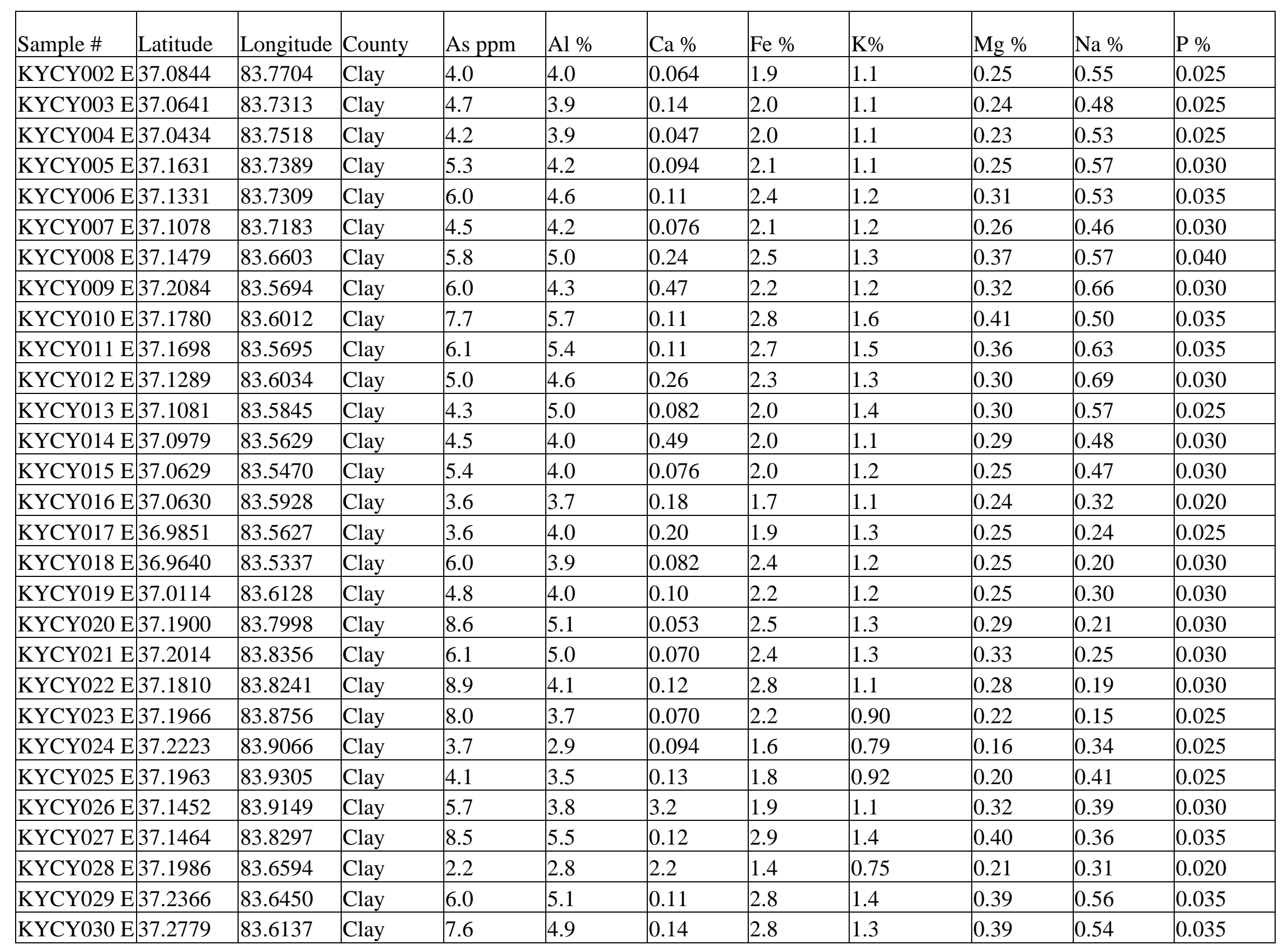




\begin{tabular}{|c|c|c|c|c|c|c|c|c|c|c|c|}
\hline KYCY032 E & 37.2421 & 83.5638 & Clay & 3.6 & 4.2 & 0.16 & 2.0 & 1.2 & 0.28 & 0.63 & 0.030 \\
\hline KYCY034 E & 37.2899 & 83.7050 & Clay & 4.6 & 3.3 & 0.088 & 1.8 & 0.81 & 0.18 & 0.39 & 0.020 \\
\hline KYCY035 E & 37.3228 & 83.7279 & Clay & 4.2 & 3.3 & 0.11 & 1.8 & 0.83 & 0.18 & 0.32 & 0.025 \\
\hline KYCY038 E & 37.2671 & 83.8761 & Clay & 6.5 & 4.1 & 0.11 & 2.0 & 1.0 & 0.21 & 0.26 & 0.025 \\
\hline KYCY039 E & 37.2834 & 83.8588 & Clay & 4.0 & 2.4 & 0.20 & 1.5 & 0.64 & 0.13 & 0.19 & 0.020 \\
\hline KYCY040 E & 37.3160 & 83.8205 & Clay & 3.8 & 2.6 & 0.04 & 1.5 & 0.68 & 0.13 & 0.24 & 0.020 \\
\hline KYCY041 E & 37.2350 & 83.7617 & Clay & 4.0 & 2.6 & 0.059 & 1.4 & 0.60 & 0.14 & 0.30 & 0.015 \\
\hline KYCY042 E & 37.2347 & 83.6974 & Clay & 7.3 & 5.0 & 0.46 & 2.9 & 1.3 & 0.36 & 0.40 & 0.035 \\
\hline KYES003 & 37.6629 & 83.9011 & Estill & 24 & 4.8 & 0.12 & 3.8 & 1.5 & 0.39 & 0.20 & 0.025 \\
\hline KYES004 & 37.6600 & 83.9265 & Estill & 23 & 3.3 & 0.80 & 3.9 & 0.96 & 0.26 & 0.22 & 0.025 \\
\hline KYES005 & 37.6145 & 83.9241 & Estill & 8.3 & 2.2 & 0.10 & 2.8 & 0.55 & 0.14 & 0.10 & 0.020 \\
\hline KYES006 & 37.5859 & 83.9200 & Estill & 5.3 & 2.0 & 0.25 & 1.4 & 0.54 & 0.15 & 0.12 & 0.015 \\
\hline KYES007 & 37.5616 & 83.9167 & Estill & 3.1 & 1.4 & 0.07 & 1.1 & 0.32 & 0.077 & 0.05 & 0.010 \\
\hline KYES017F & 37.6628 & 84.0087 & Estill & 28 & 3.7 & 0.25 & 3.7 & 1.3 & 0.31 & 0.23 & 0.030 \\
\hline KYES024F & & & Estill & 12 & 4.3 & 0.14 & 2.5 & 1.4 & 0.37 & 0.46 & 0.015 \\
\hline KYES030F & 37.8049 & 84.0498 & Estill & 29 & 4.1 & 5.9 & 4.5 & 1.8 & 2.9 & 0.13 & 0.080 \\
\hline KYES034 & 37.6809 & 83.9116 & Estill & 22 & 2.8 & 0.84 & 2.7 & 0.93 & 0.26 & 0.19 & 0.027 \\
\hline KYES036 & 37.6691 & 83.8390 & Estill & 14 & 3.6 & 1.1 & 2.4 & 1.1 & 0.31 & 0.22 & 0.025 \\
\hline
\end{tabular}




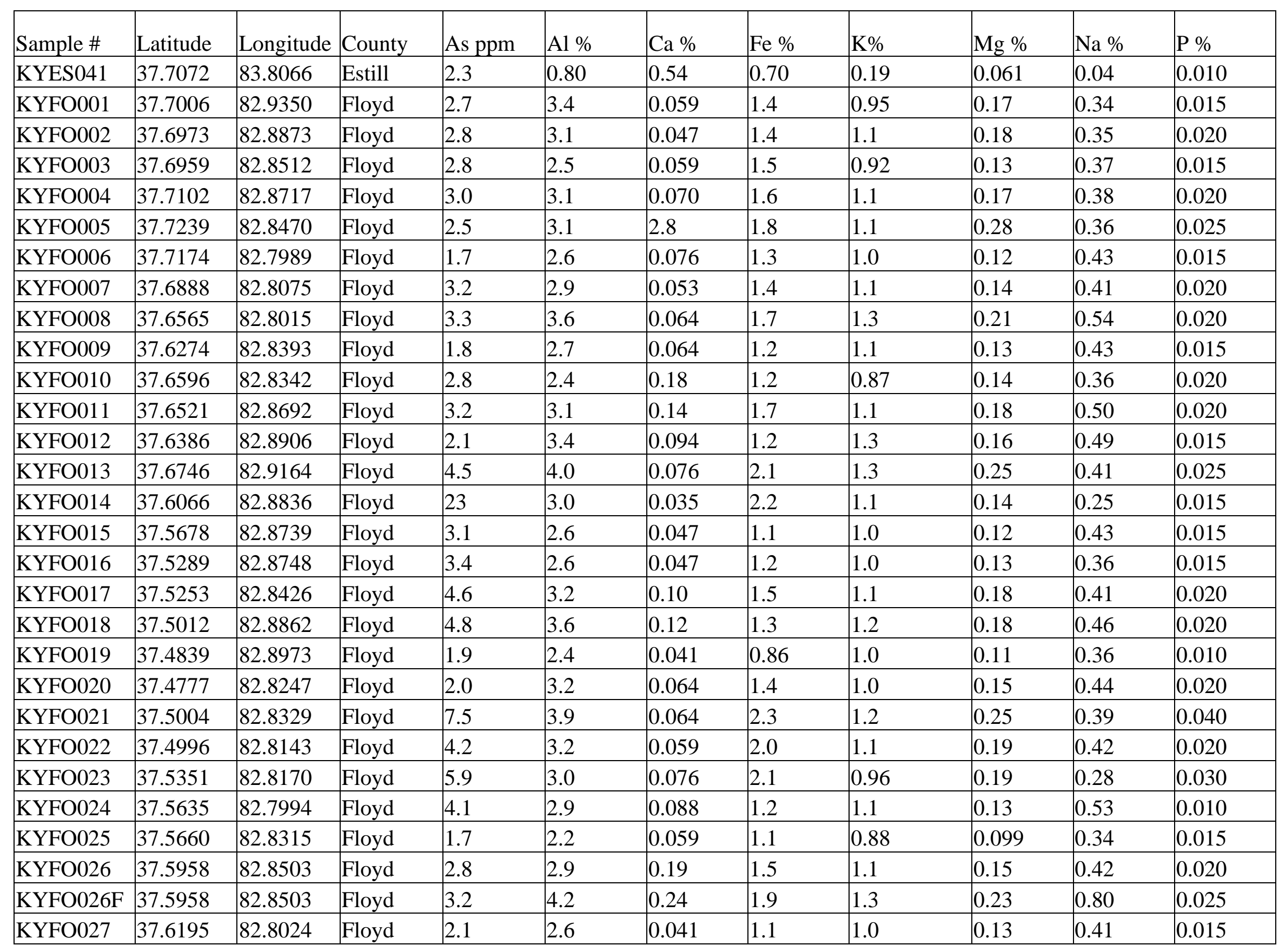




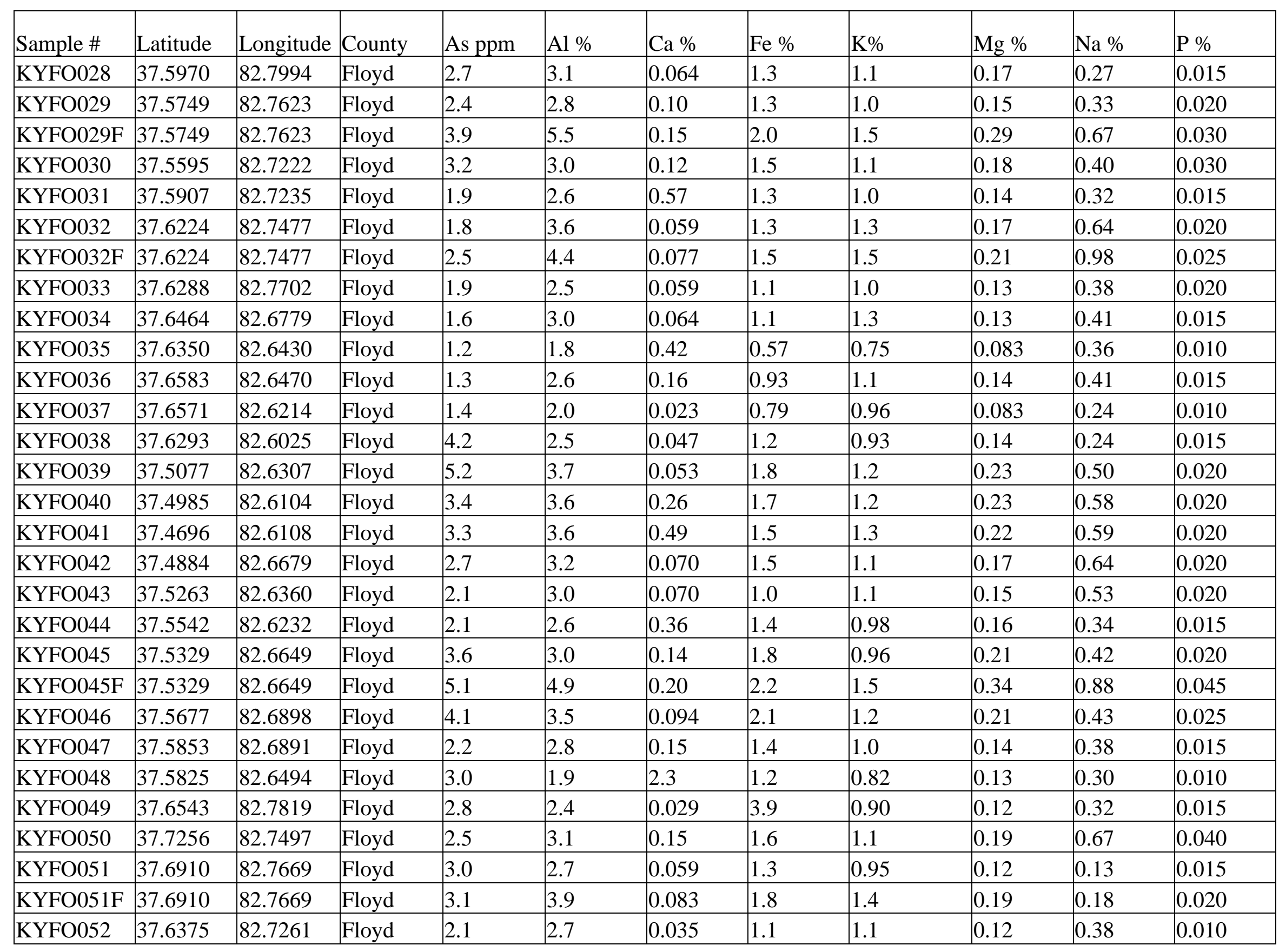




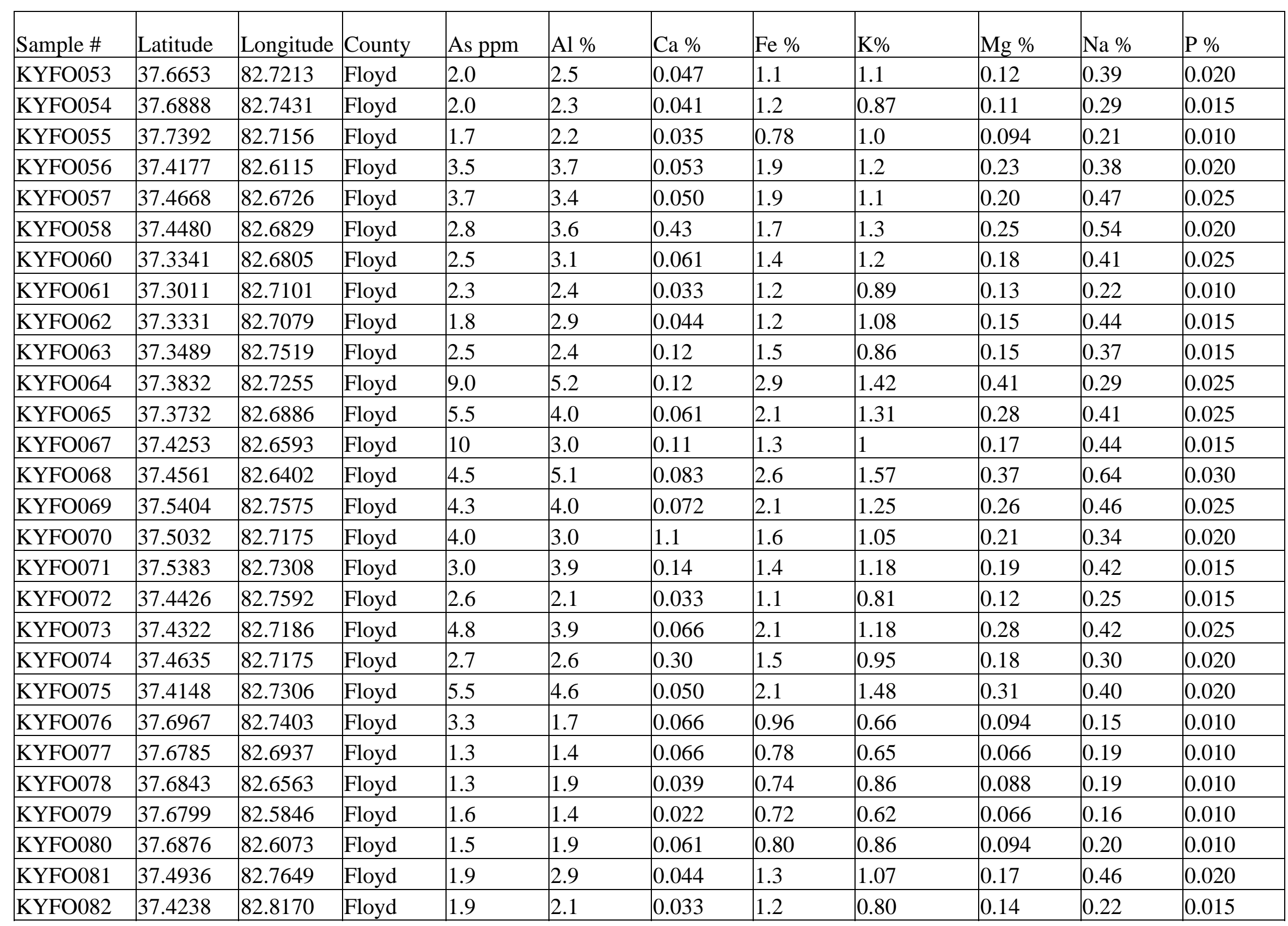




\begin{tabular}{|c|c|c|c|c|c|c|c|c|c|c|c|}
\hline KYHL001E & 36.8637 & 83.3338 & Harlan & 4.0 & 3.0 & 1.2 & 1.6 & 0.86 & 0.26 & 0.21 & 0.025 \\
\hline KYHL003E & 36.8338 & 83.3745 & Harlan & 5.6 & 5.2 & 0.11 & 2.8 & 1.56 & 0.40 & 0.51 & 0.035 \\
\hline KYHL004E & 36.8382 & 83.4159 & Harlan & 3.7 & 1.7 & 0.66 & 1.5 & 0.51 & 0.18 & 0.15 & 0.020 \\
\hline KYHL007E & 36.9030 & 83.2466 & Harlan & 2.2 & 2.0 & 0.12 & 0.99 & 0.43 & 0.11 & 0.045 & 0.050 \\
\hline KYHL008E & 36.9365 & 83.1412 & Harlan & 2.4 & 2.0 & 0.028 & 1.0 & 0.39 & 0.11 & 0.035 & 0.030 \\
\hline KYHL009E & 36.9553 & 83.0776 & Harlan & 1.2 & 1.2 & 0.022 & 0.60 & 0.35 & 0.077 & 0.020 & 0.010 \\
\hline KYHL010E & 36.9954 & 82.9201 & Harlan & 3.3 & 4.8 & 0.14 & 2.1 & 1.53 & 0.36 & 0.55 & 0.030 \\
\hline KYHL011E & 36.9616 & 82.9473 & Harlan & 5.9 & 4.7 & 0.29 & 2.3 & 1.54 & 0.36 & 0.40 & 0.030 \\
\hline KYHL015E & 36.9303 & 83.3096 & Harlan & 2.8 & 2.3 & 0.088 & 1.2 & 0.73 & 0.14 & 0.080 & 0.020 \\
\hline KYHL016E & 36.8541 & 83.4783 & Harlan & 5.1 & 4.1 & 0.099 & 2.5 & 1.06 & 0.22 & 0.080 & 0.025 \\
\hline KYHL017E & 36.9208 & 83.3675 & Harlan & 5.0 & 3.8 & 0.094 & 2.0 & 0.99 & 0.20 & 0.055 & 0.020 \\
\hline KYHL018E & 36.9648 & 83.2012 & Harlan & 3.5 & 2.5 & 0.072 & 1.5 & 0.91 & 0.14 & 0.060 & 0.015 \\
\hline KYHL019E & 36.9855 & 83.2055 & Harlan & 3.2 & 3.7 & 0.072 & 1.7 & 1.5 & 0.22 & 0.075 & 0.025 \\
\hline KYHL020E & 36.9968 & 83.1691 & Harlan & 1.8 & 1.9 & 0.072 & 0.66 & 0.99 & 0.077 & 0.040 & 0.010 \\
\hline KYHL021E & 36.7697 & 83.3230 & Harlan & 3.7 & 4.3 & 0.57 & 1.9 & 1.3 & 0.29 & 0.51 & 0.020 \\
\hline KYHL022E & 36.7495 & 83.3534 & Harlan & 5.7 & 4.8 & 0.12 & 2.4 & 1.5 & 0.36 & 0.36 & 0.030 \\
\hline KYHL023E & 36.8062 & 83.3042 & Harlan & 9.5 & 5.3 & 0.49 & 2.9 & 1.7 & 0.42 & 0.45 & 0.030 \\
\hline KYHL024E & 36.8141 & 83.2522 & Harlan & 6.9 & 4.5 & 1.5 & 2.1 & 1.4 & 0.36 & 0.51 & 0.025 \\
\hline
\end{tabular}




\begin{tabular}{|c|c|c|c|c|c|c|c|c|c|c|c|}
\hline KYHL030E & 36.9242 & 83.0678 & Harlan & 6.0 & 5.0 & 0.20 & 2.4 & 1.3 & 0.32 & 0.34 & 0.030 \\
\hline KYHL032E & 36.8859 & 83.0224 & Harlan & 4.4 & 3.8 & 1.0 & 2.0 & 1.2 & 0.30 & 0.30 & 0.030 \\
\hline KYHL033E & 36.8683 & 83.0086 & Harlan & 4.5 & 4.4 & 0.14 & 2.2 & 1.3 & 0.32 & 0.60 & 0.030 \\
\hline KYHL036E & 36.7490 & 83.4790 & Harlan & 4.0 & 3.9 & 0.061 & 2.1 & 1.1 & 0.27 & 0.35 & 0.030 \\
\hline KYHL037E & 36.7519 & 83.4459 & Harlan & 5.5 & 4.8 & 0.099 & 2.5 & 1.5 & 0.37 & 0.37 & 0.030 \\
\hline KYHL038E & 36.7240 & 83.4547 & Harlan & 14 & 5.9 & 0.099 & 3.5 & 1.7 & 0.43 & 0.32 & 0.040 \\
\hline KYHL039E & 36.6922 & 83.3945 & Harlan & 3.3 & 2.8 & 0.055 & 1.6 & 0.87 & 0.18 & 0.23 & 0.020 \\
\hline KYHL040E & 36.7081 & 83.3610 & Harlan & 6.6 & 3.5 & 0.099 & 2.2 & 1.1 & 0.24 & 0.37 & 0.025 \\
\hline KYJA004 & 37.4527 & 83.9702 & Jackson & 3.8 & 2.1 & 0.022 & 1.61 & 0.45 & 0.099 & 0.035 & 0.015 \\
\hline KYJA005 & 37.4665 & 83.9290 & Jackson & 2.8 & 1.0 & 0.017 & 0.93 & 0.21 & 0.044 & 0.020 & 0.010 \\
\hline KYJA006 & 37.4902 & 83.9605 & Jackson & 1.5 & 0.6 & 0.017 & 0.47 & 0.13 & 0.028 & 0.010 & 0.005 \\
\hline KYJA007 & 37.5045 & 83.9385 & Jackson & 2.9 & 1.0 & 0.050 & 0.93 & 0.23 & 0.061 & 0.015 & 0.010 \\
\hline KYJA008 & 37.5067 & 84.0364 & Jackson & 2.3 & 0.83 & 0.050 & 1.0 & 0.15 & 0.039 & 0.010 & 0.010 \\
\hline KYJA009 & 37.4786 & 84.0433 & Jackson & 2.6 & 1.1 & 0.028 & 0.97 & 0.18 & 0.039 & 0.015 & 0.010 \\
\hline KYJA009F & 37.4786 & 84.0433 & Jackson & 3.3 & 1.0 & 0.039 & 1.1 & 0.12 & 0.052 & 0.005 & 0.016 \\
\hline KYJA010 & 37.4603 & 84.0189 & Jackson & 2.5 & 1.0 & 0.044 & 0.96 & 0.20 & 0.044 & 0.015 & 0.010 \\
\hline KYJA011 & 37.3132 & 84.0227 & Jackson & 11 & 5.6 & 0.099 & 6.1 & 1.3 & 0.31 & 0.11 & 0.045 \\
\hline KYJA012 & 37.2734 & 84.0304 & Jackson & 6.9 & 4.7 & 0.099 & 4.9 & 1.1 & 0.26 & 0.12 & 0.065 \\
\hline
\end{tabular}




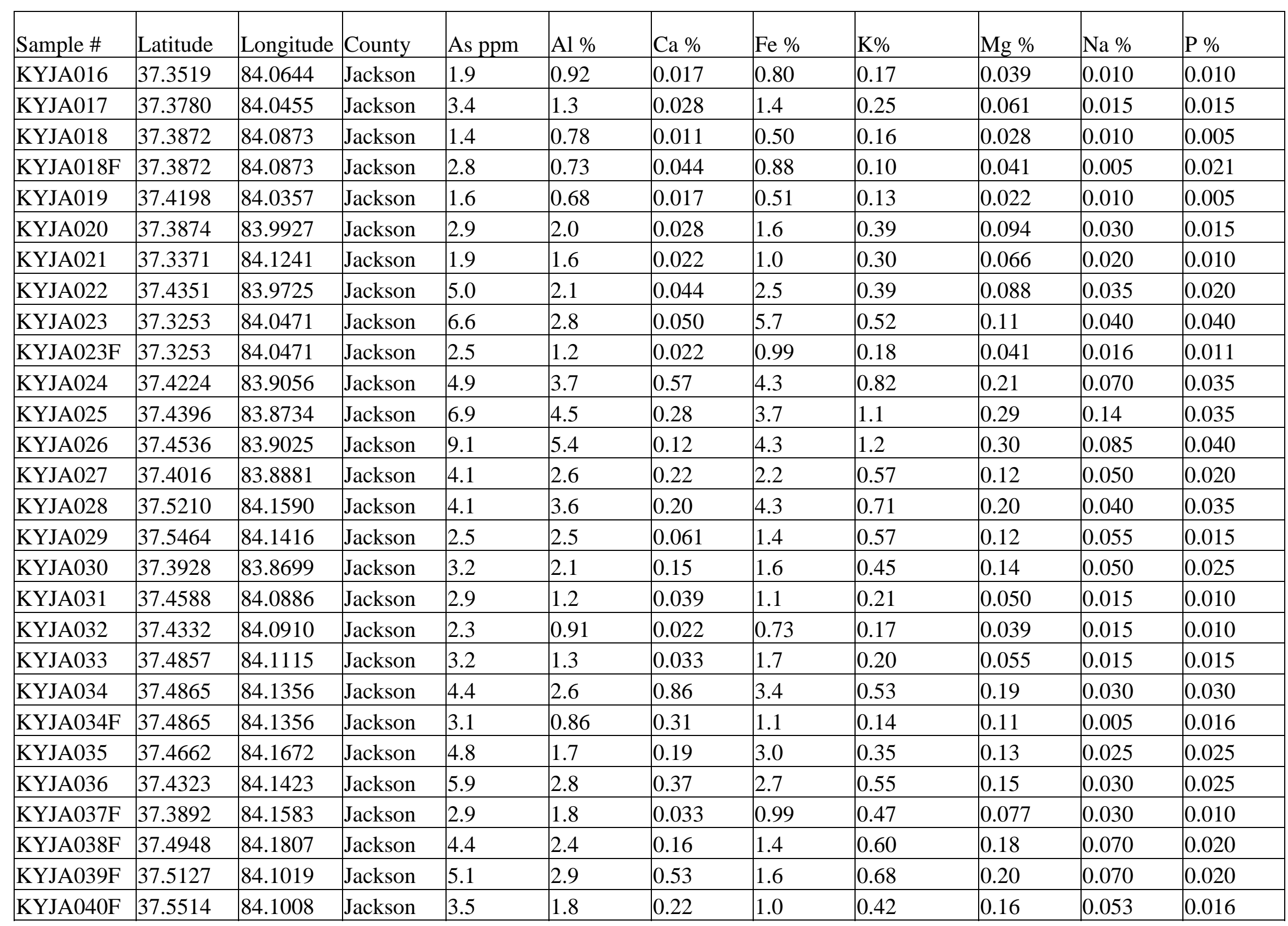




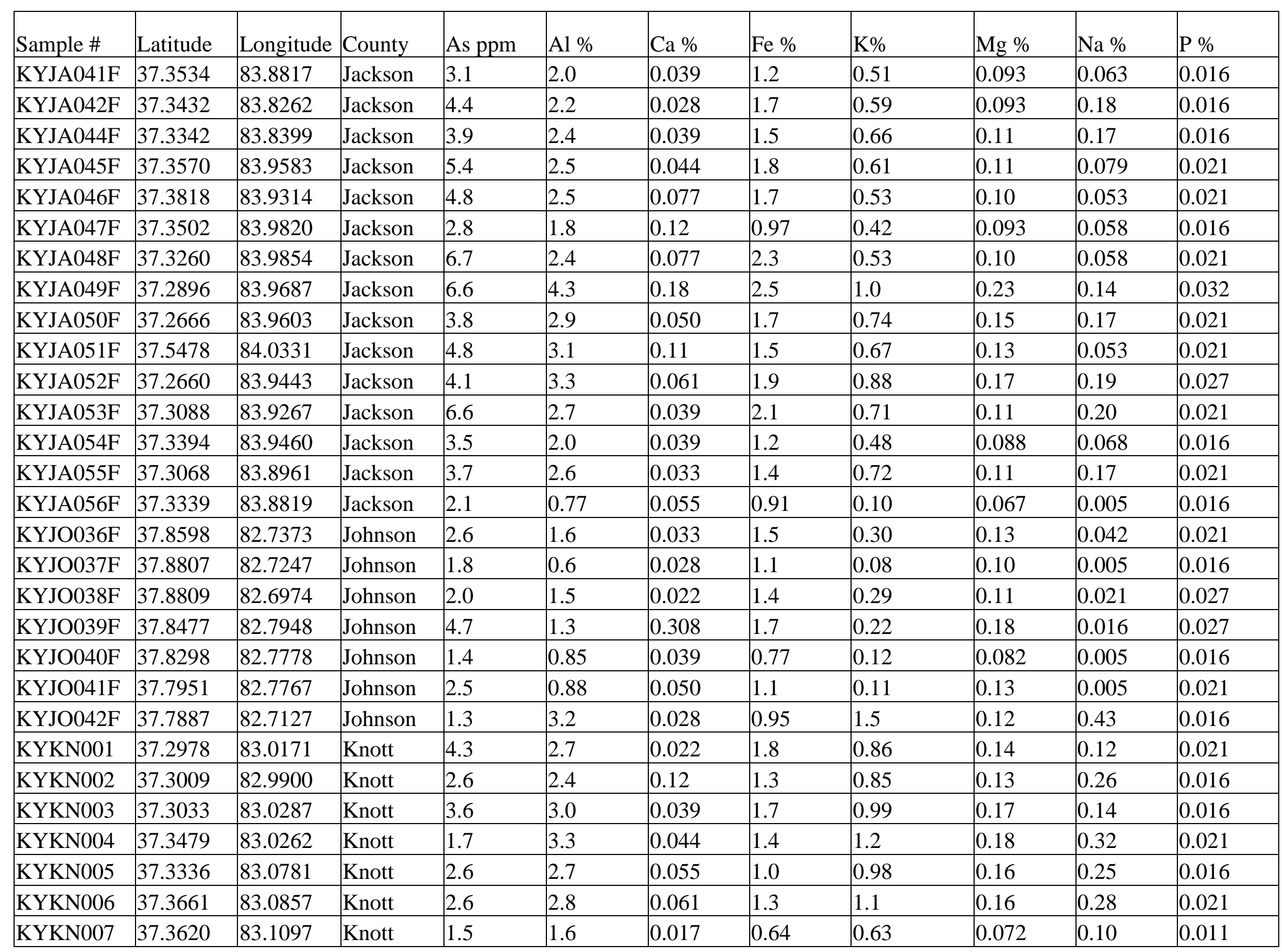




\begin{tabular}{|c|c|c|c|c|c|c|c|c|c|c|c|}
\hline KYKN009 & 37.2797 & 82.9238 & Knott & 3.7 & 4.5 & 0.094 & 2.5 & 1.4 & 0.33 & 0.45 & 0.038 \\
\hline KYKN011 & 37.2614 & 82.8473 & Knott & 2.5 & 2.9 & 0.044 & 1.3 & 0.97 & 0.15 & 0.39 & 0.016 \\
\hline KYKN012 & 37.2605 & 82.8792 & Knott & 5.6 & 4.2 & 0.077 & 2.9 & 1.2 & 0.29 & 0.31 & 0.032 \\
\hline KYKN015 & 37.3975 & 82.9407 & Knott & 2.1 & 1.9 & 0.028 & 0.94 & 0.75 & 0.098 & 0.16 & 0.011 \\
\hline KYKN016 & 37.4466 & 82.9913 & Knott & 0.9 & 2.2 & 0.033 & 0.87 & 0.93 & 0.11 & 0.28 & 0.016 \\
\hline KYKN017 & 37.4575 & 83.0307 & Knott & 0.9 & 1.5 & 0.017 & 0.51 & 0.70 & 0.062 & 0.16 & 0.011 \\
\hline KYKN018 & 37.4726 & 83.0613 & Knott & 2.1 & 2.4 & 0.028 & 0.83 & 0.97 & 0.11 & 0.22 & 0.011 \\
\hline KYKN019 & 37.5067 & 83.0646 & Knott & 2.2 & 2.3 & 0.033 & 0.96 & 0.99 & 0.11 & 0.18 & 0.016 \\
\hline KYKN023 & 37.2246 & 82.9560 & Knott & 3.7 & 3.5 & 0.050 & 2.1 & 1.1 & 0.22 & 0.29 & 0.021 \\
\hline KYKN024 & 37.2257 & 82.9267 & Knott & 4.5 & 4.0 & 0.055 & 2.5 & 1.1 & 0.24 & 0.23 & 0.027 \\
\hline KYKN025 & 37.1941 & 82.9811 & Knott & 3.7 & 2.8 & 0.050 & 2.0 & 0.81 & 0.17 & 0.12 & 0.021 \\
\hline KYKN026 & 37.2409 & 82.9130 & Knott & 3.5 & 3.3 & 0.11 & 3.9 & 1.0 & 0.24 & 0.29 & 0.043 \\
\hline KYKN027 & 37.2634 & 83.0890 & Knott & 8.2 & 3.3 & 0.028 & 2.3 & 1.0 & 0.20 & 0.14 & 0.021 \\
\hline KYKN028 & 37.2733 & 83.0957 & Knott & 4.9 & 2.6 & 0.077 & 1.7 & 0.84 & 0.15 & 0.079 & 0.016 \\
\hline KYKN029 & 37.3256 & 83.0981 & Knott & 1.8 & 1.9 & 0.033 & 0.80 & 0.77 & 0.088 & 0.19 & 0.011 \\
\hline KYKN030 & 37.2934 & 83.0808 & Knott & 11 & 2.3 & 0.23 & 1.7 & 0.73 & 0.14 & 0.079 & 0.021 \\
\hline KYKN031 & 37.3279 & 83.0198 & Knott & 1.8 & 1.9 & 0.028 & 1.0 & 0.75 & 0.098 & 0.17 & 0.016 \\
\hline KYKN032 & 37.3538 & 82.9725 & Knott & 4.1 & 3.4 & 0.050 & 1.4 & 1.3 & 0.20 & 0.44 & 0.016 \\
\hline
\end{tabular}




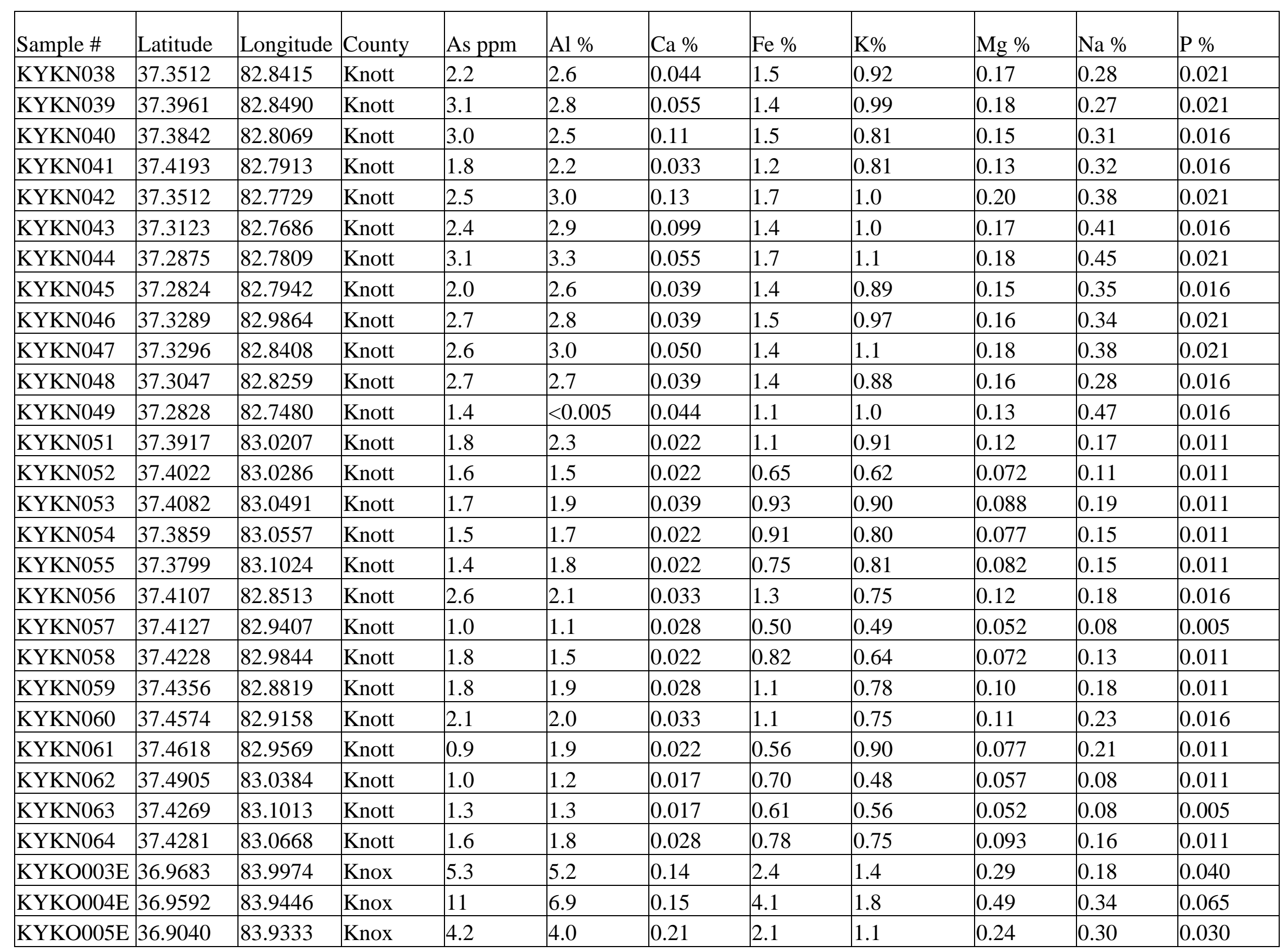




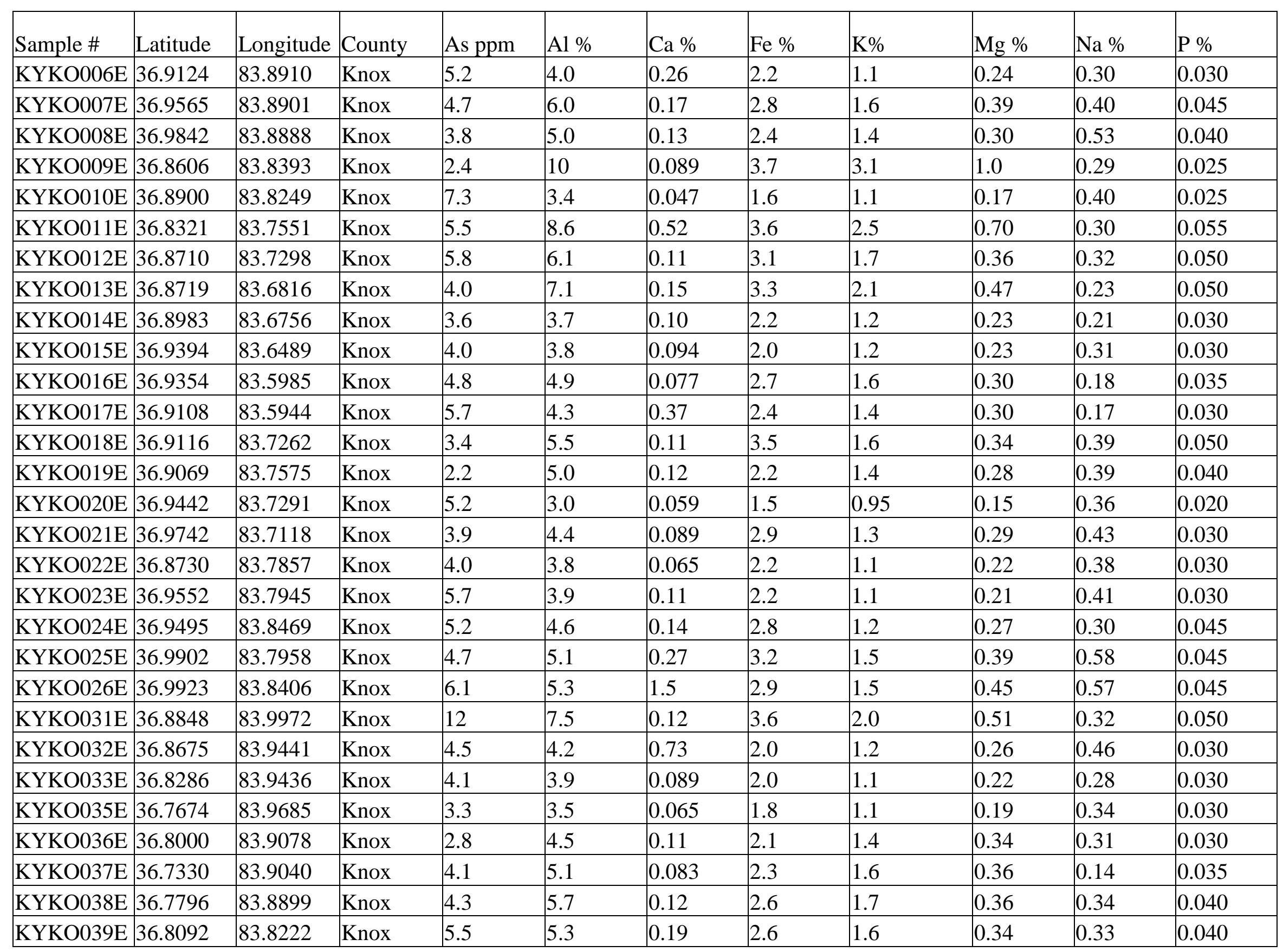




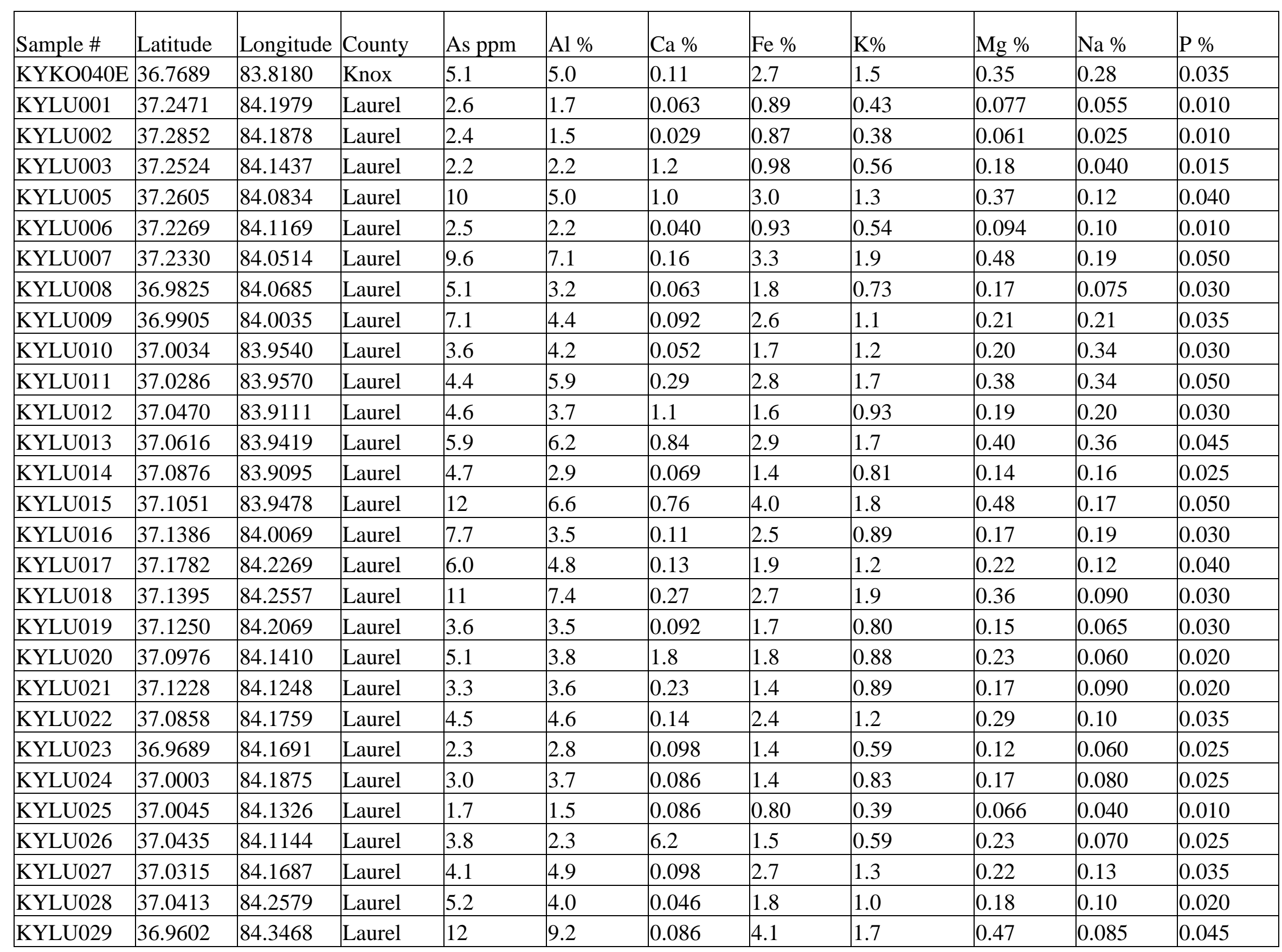




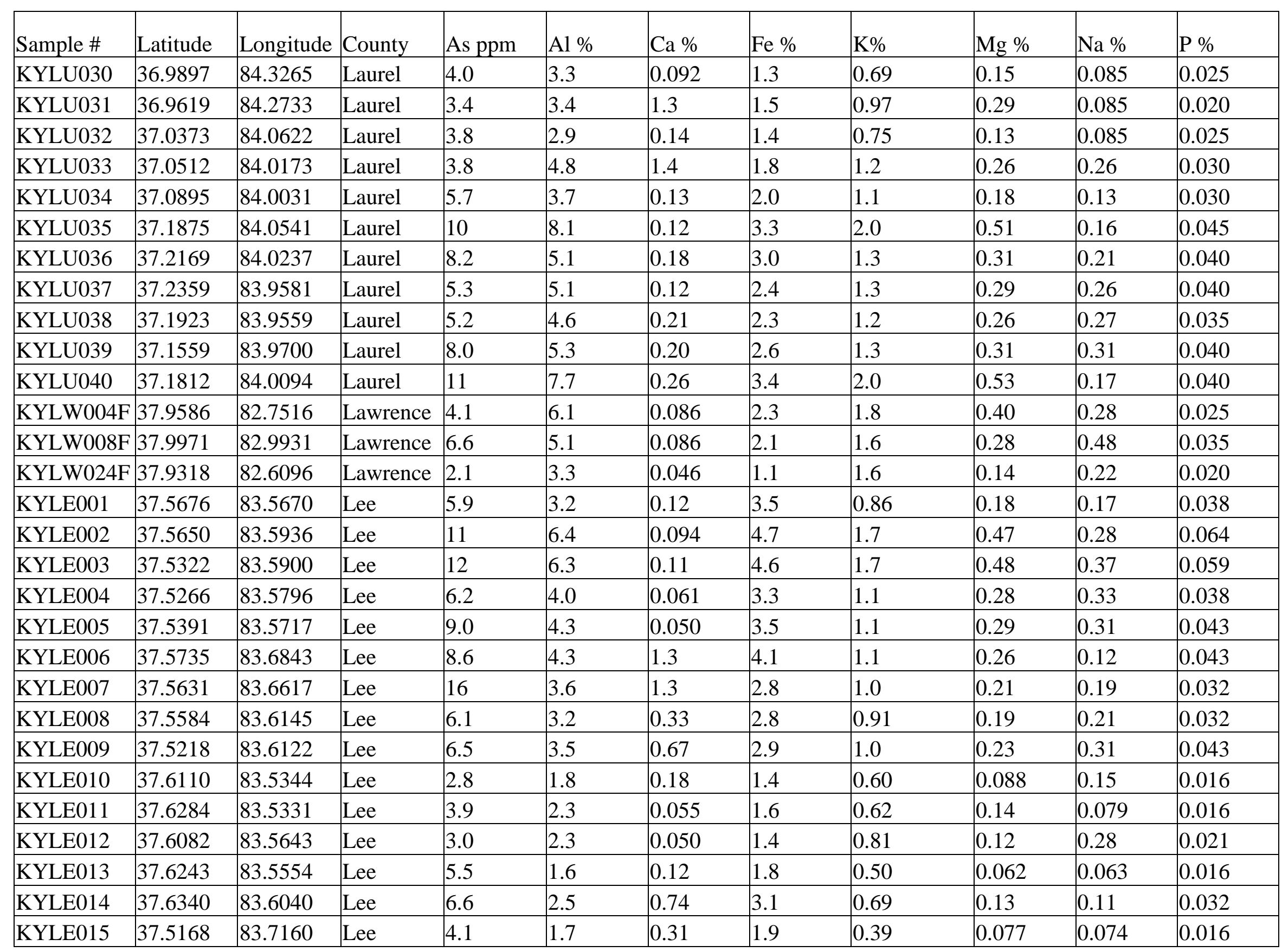




\begin{tabular}{|c|c|c|c|c|c|c|c|c|c|c|c|}
\hline KYLE017 & 37.5209 & 83.6936 & Lee & 8.4 & 5.0 & 0.13 & 4.7 & 1.3 & 0.31 & 0.18 & 0.050 \\
\hline KYLE019 & 37.5103 & 83.7460 & Lee & 5.7 & 3.1 & 0.099 & 2.9 & 0.79 & 0.19 & 0.14 & 0.033 \\
\hline KYLE020 & 37.5354 & 83.7743 & Lee & 11 & 4.0 & 0.16 & 3.9 & 0.94 & 0.24 & 0.11 & 0.039 \\
\hline KYLE023 & 37.4991 & 83.8440 & Lee & 9.2 & 3.7 & 0.061 & 4.0 & 0.85 & 0.19 & 0.070 & 0.039 \\
\hline KYLE024 & 37.4966 & 83.8027 & Lee & 9.4 & 4.5 & 0.34 & 5.4 & 1.0 & 0.26 & 0.085 & 0.055 \\
\hline KYLE025 & 37.5397 & 83.8107 & Lee & 16 & 5.0 & 0.20 & 4.9 & 1.1 & 0.30 & 0.065 & 0.044 \\
\hline KYLE026 & 37.5292 & 83.8165 & Lee & 8.3 & 4.6 & 0.17 & 5.3 & 1.0 & 0.25 & 0.060 & 0.050 \\
\hline KYLE027 & 37.5097 & 83.8265 & Lee & 11 & 6.2 & 0.072 & 5.3 & 1.3 & 0.33 & 0.075 & 0.050 \\
\hline KYLE031 & 37.6356 & 83.7058 & Lee & 9.0 & 3.9 & 0.077 & 3.9 & 0.95 & 0.16 & 0.090 & 0.033 \\
\hline KYLE032 & 37.6667 & 83.6978 & Lee & 3.1 & 2.0 & 0.237 & 1.3 & 0.49 & 0.12 & 0.025 & 0.017 \\
\hline KYLE033 & 37.6728 & 83.7253 & Lee & 2.8 & 0.67 & 0.066 & 0.59 & 0.16 & 0.039 & 0.025 & 0.006 \\
\hline KYLE034 & 37.6937 & 83.6987 & Lee & 2.3 & 0.67 & 0.077 & 0.63 & 0.18 & 0.033 & 0.025 & 0.006 \\
\hline KYLE035 & 37.6930 & 83.7402 & Lee & 1.1 & 0.28 & 1.2 & 0.30 & 0.07 & 0.022 & 0.025 & 0.006 \\
\hline KYLE036 & 37.6685 & 83.7570 & Lee & 0.6 & 0.23 & 0.033 & 0.20 & 0.06 & 0.011 & 0.010 & 0.006 \\
\hline KYLE037 & 37.6705 & 83.7672 & Lee & 0.9 & 0.32 & 0.011 & 0.31 & 0.07 & 0.011 & 0.005 & 0.006 \\
\hline KYLE038 & 37.6512 & 83.8019 & Lee & 1.1 & 0.32 & 0.022 & 0.45 & 0.07 & 0.017 & 0.010 & 0.006 \\
\hline KYLE039 & 37.6334 & 83.8068 & Lee & 3.3 & 0.60 & 0.50 & 0.73 & 0.14 & 0.033 & 0.010 & 0.006 \\
\hline KYLE040 & 37.5649 & 83.7305 & Lee & 17 & 4.8 & 0.050 & 5.1 & 1.1 & 0.26 & 0.085 & 0.050 \\
\hline
\end{tabular}




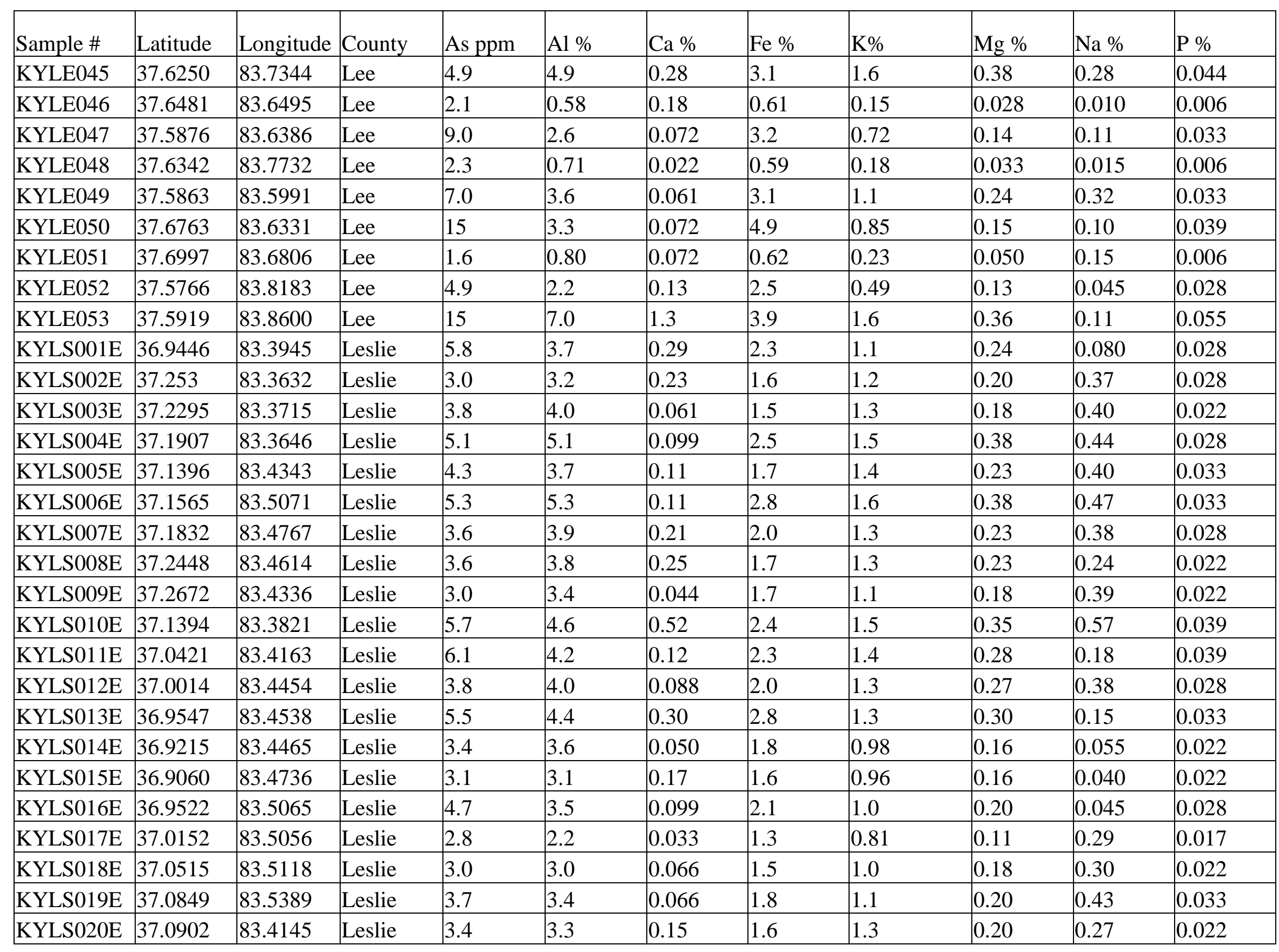




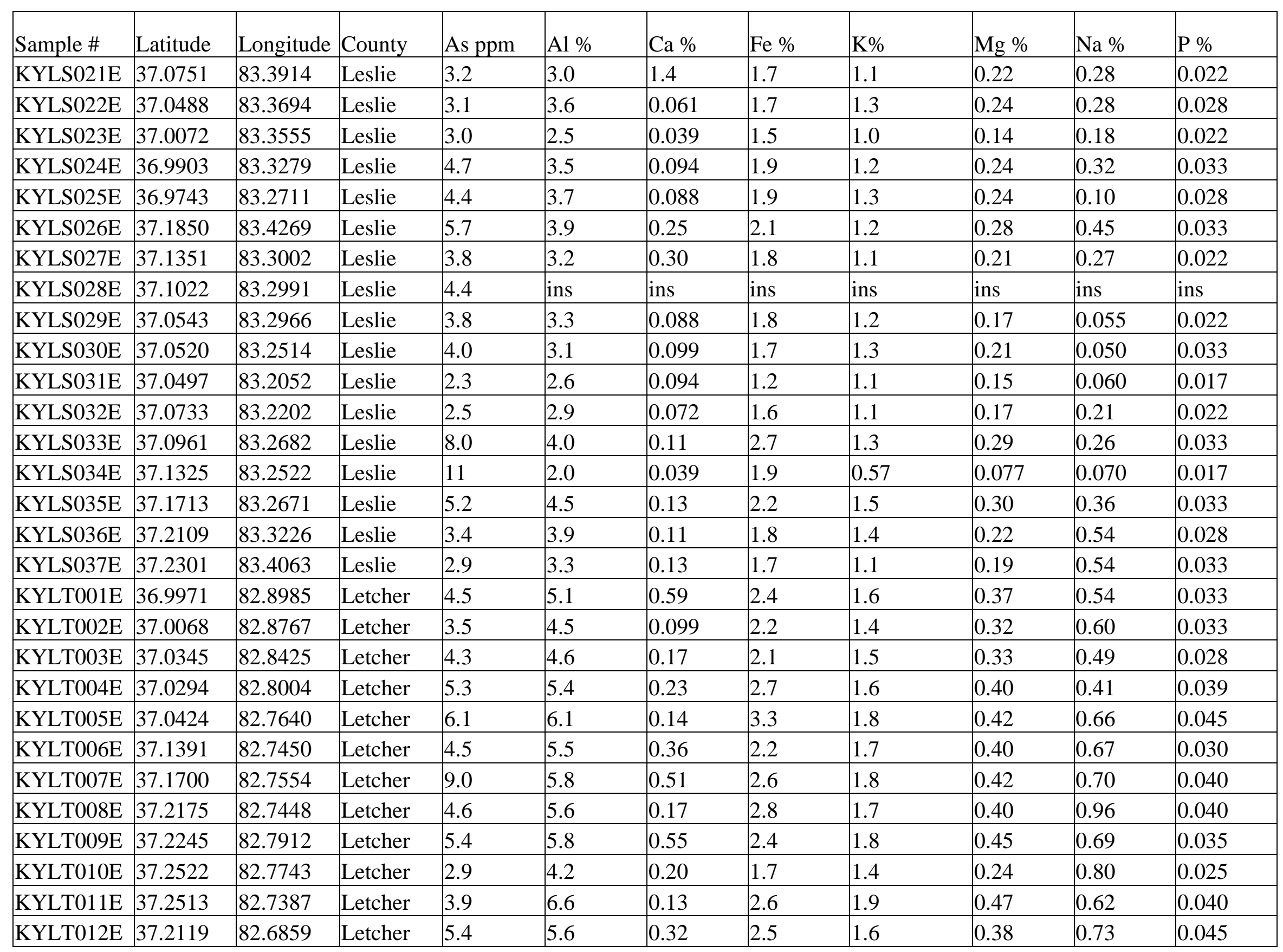




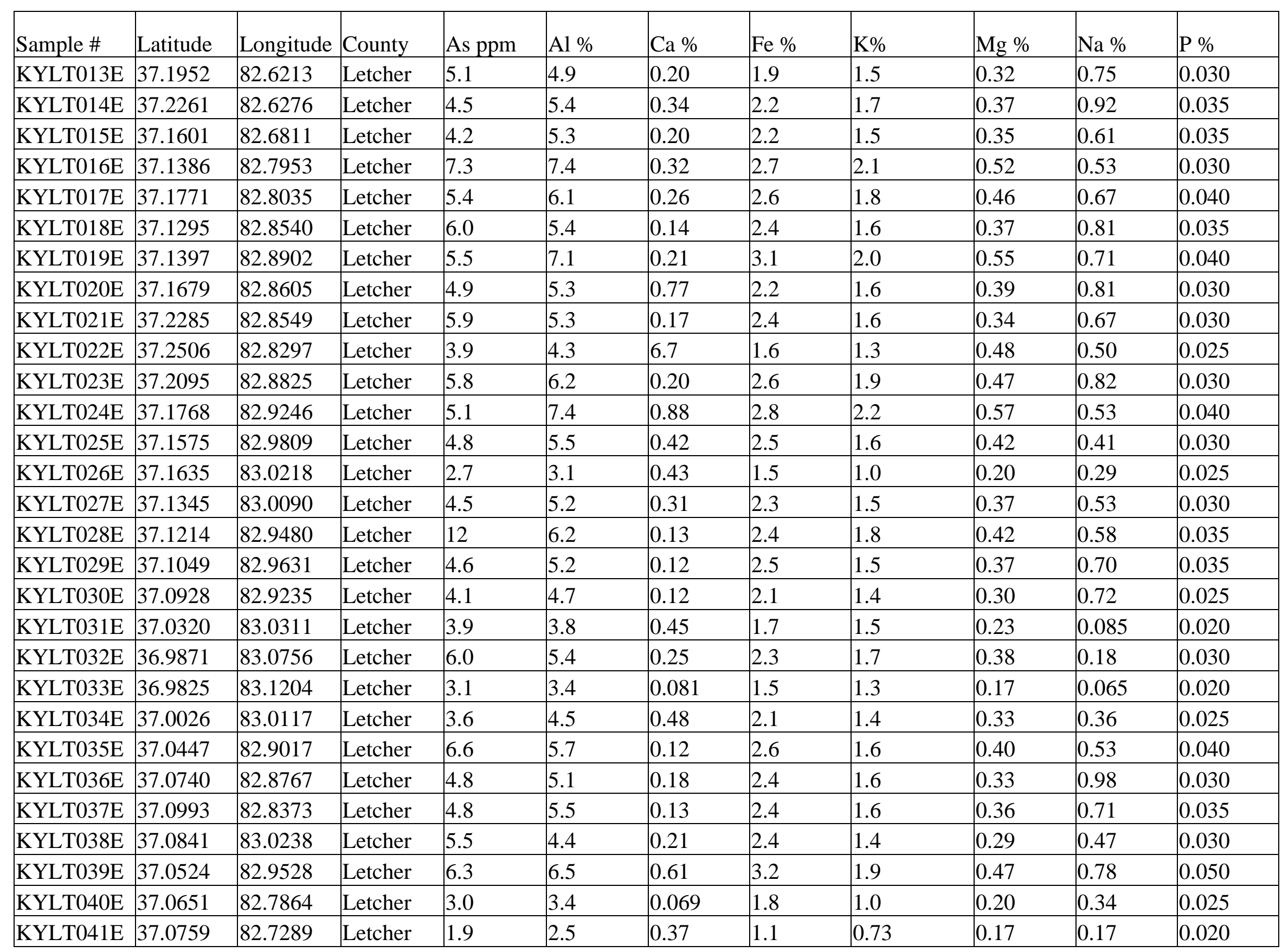




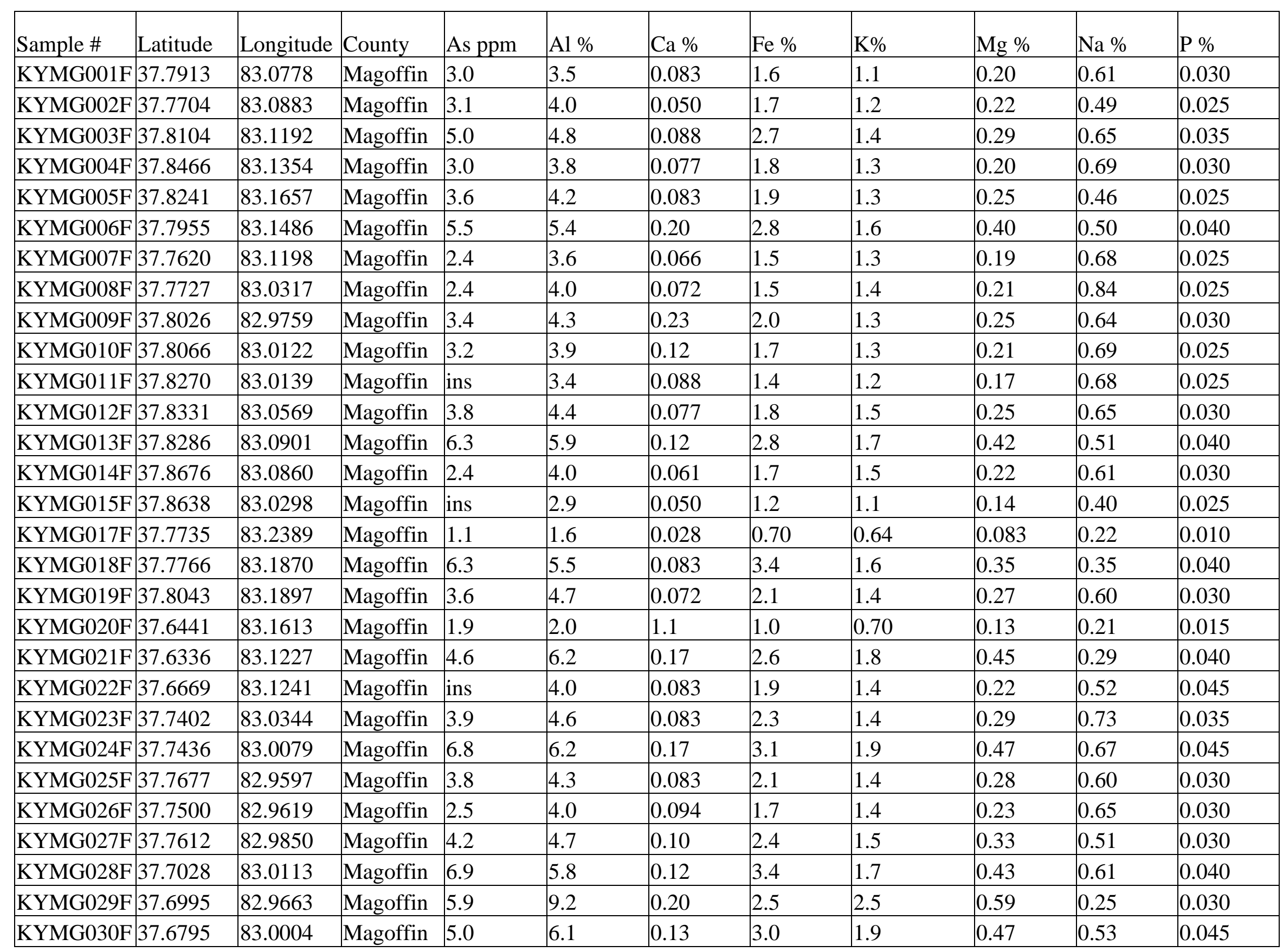




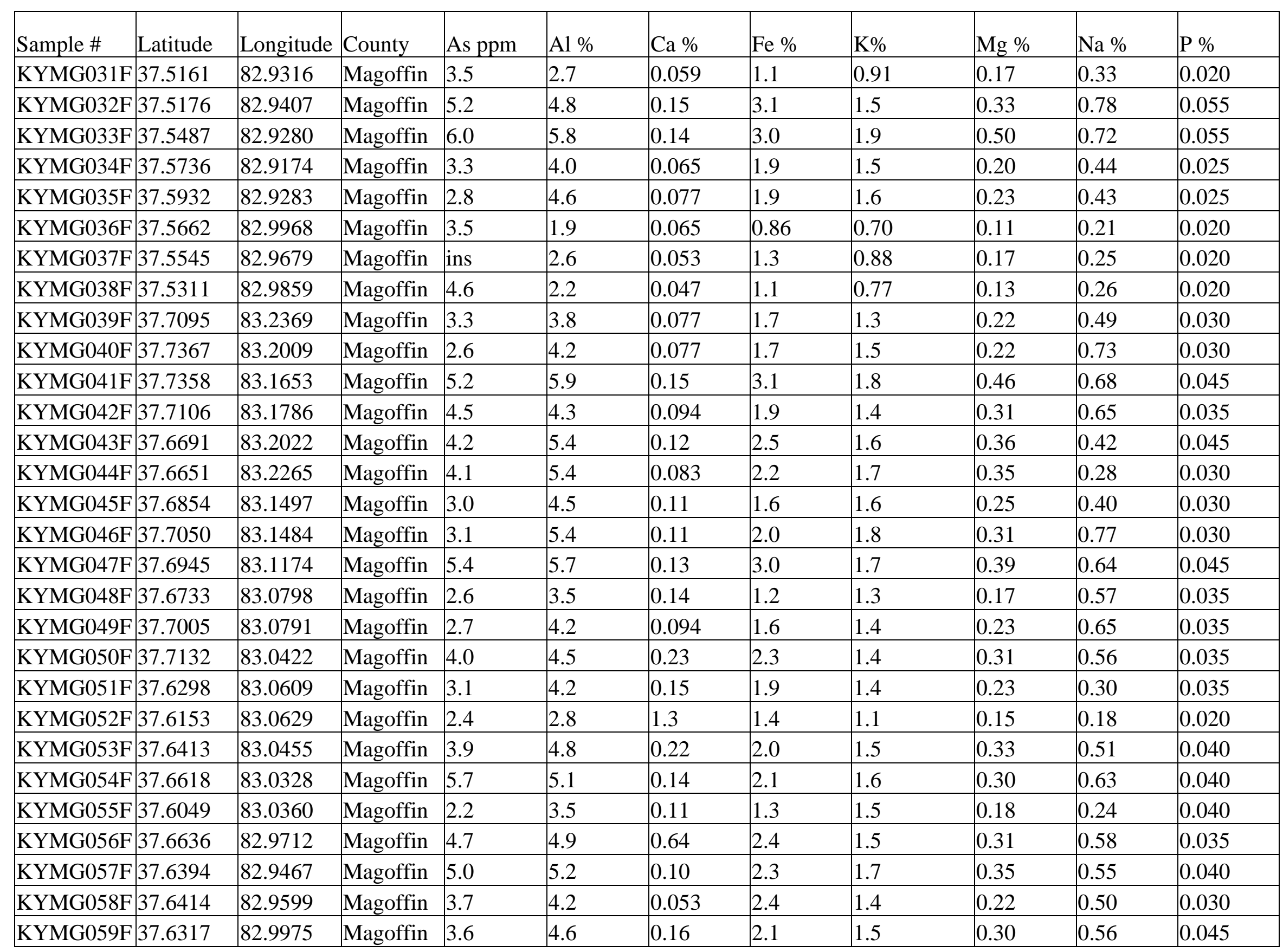




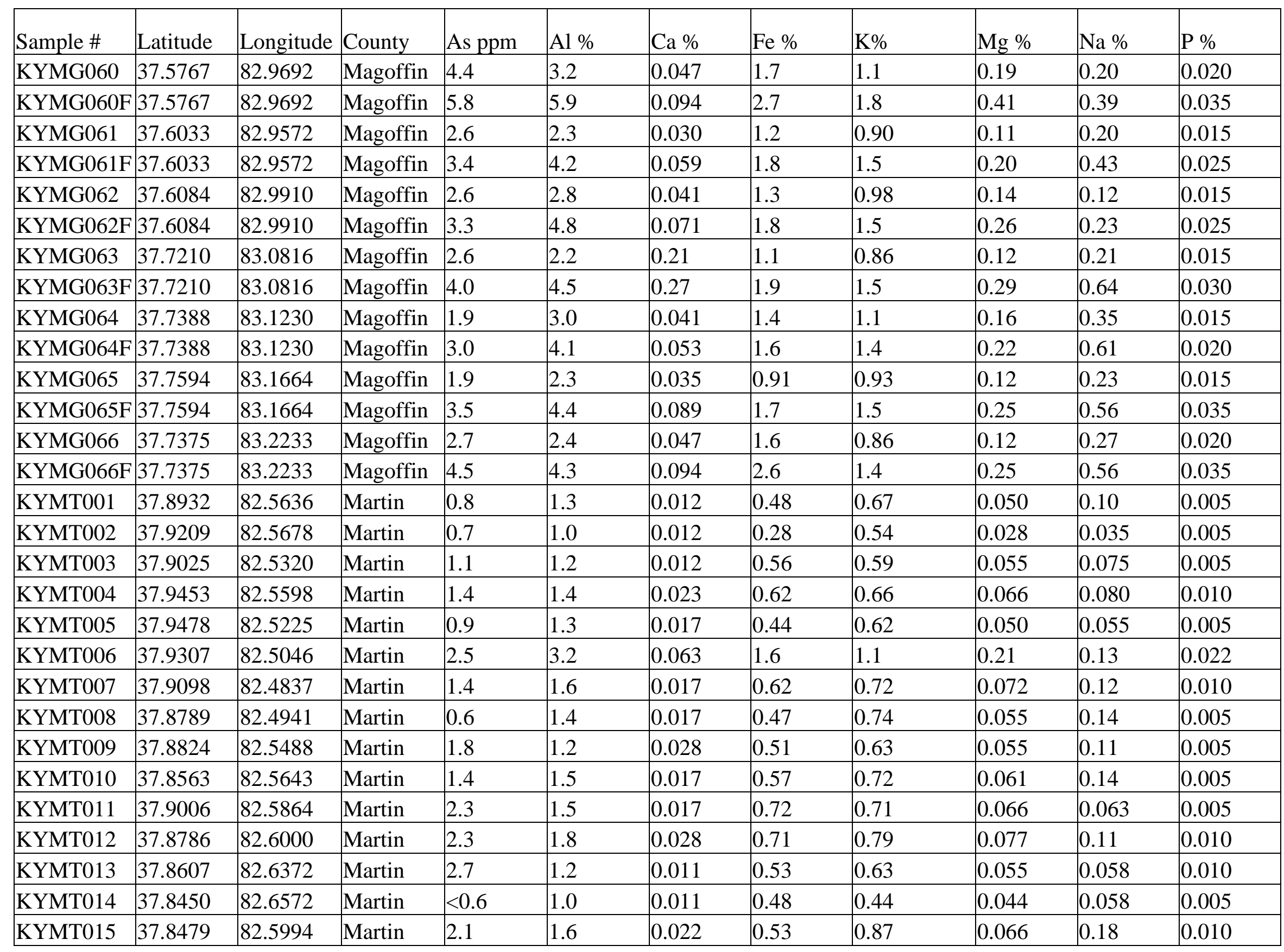




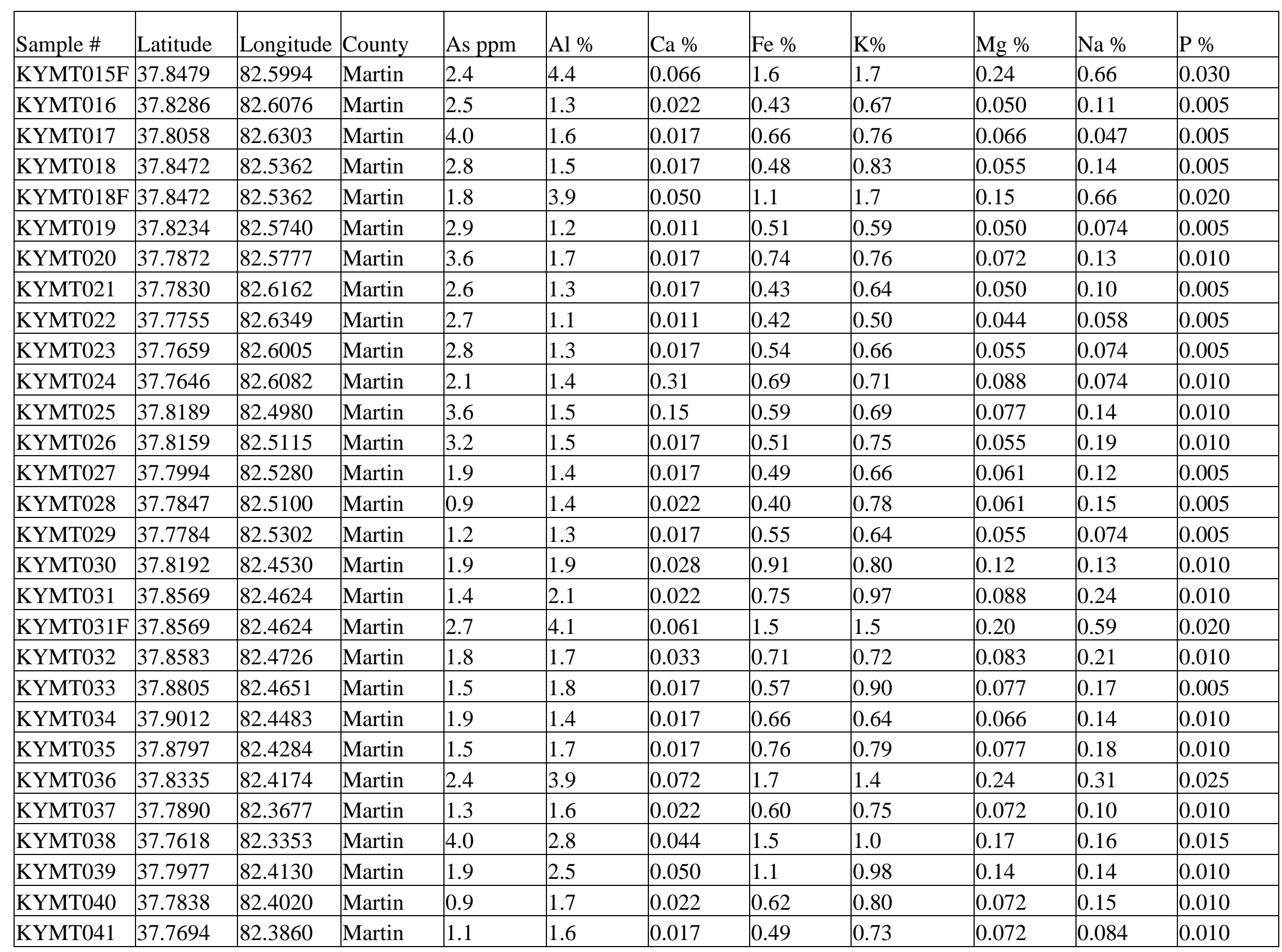




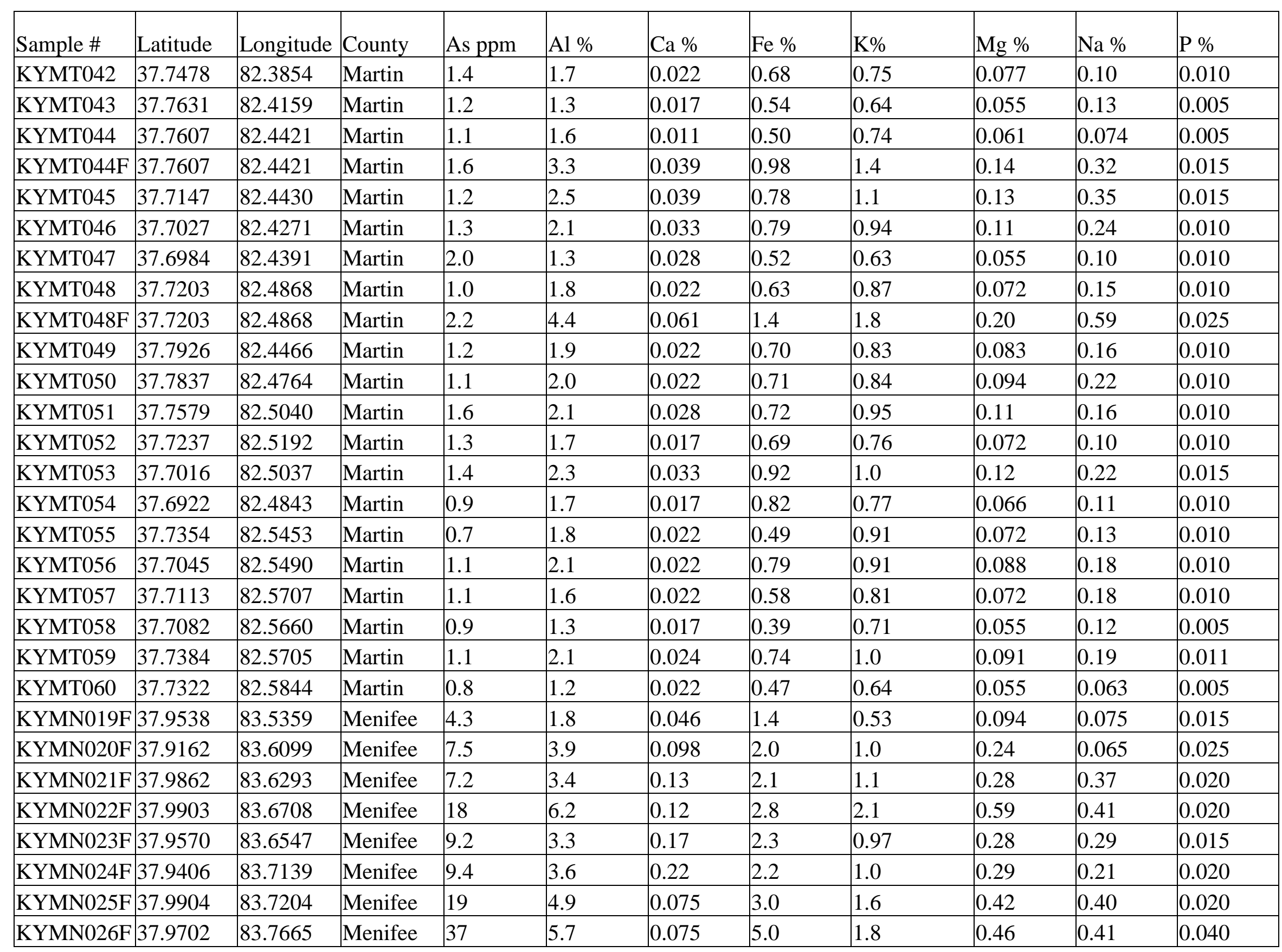




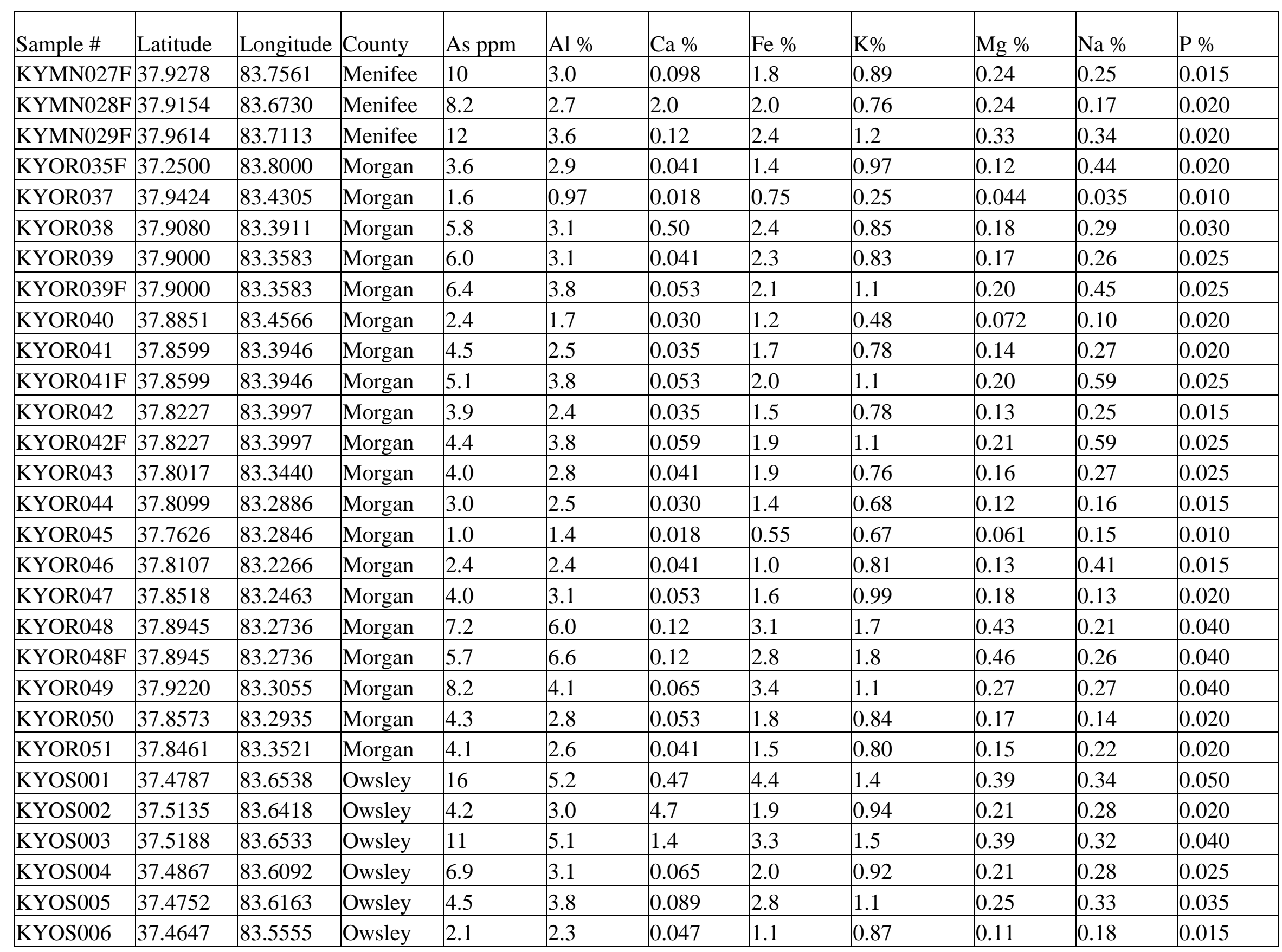




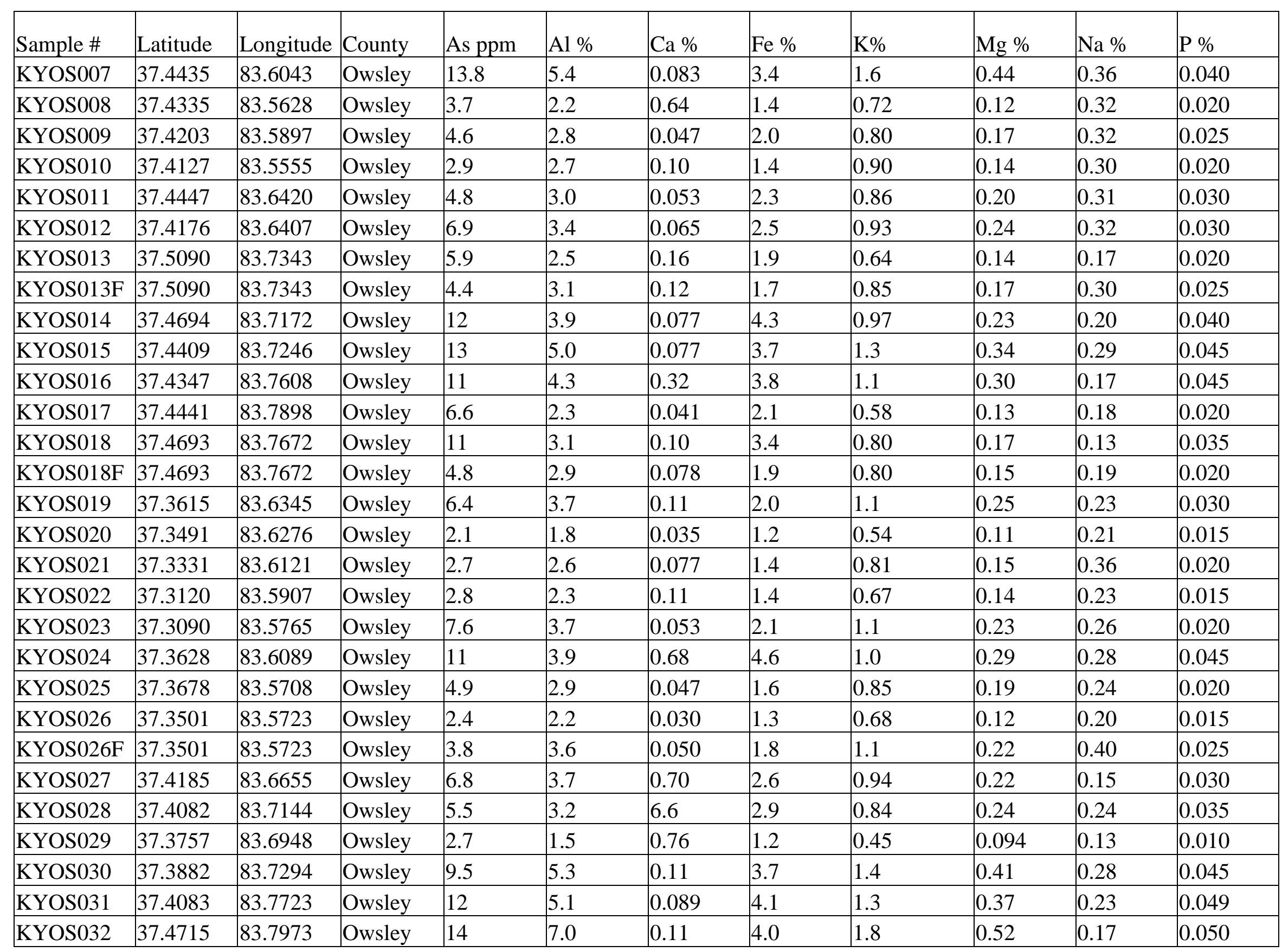




\begin{tabular}{|c|c|c|c|c|c|c|c|c|c|c|c|}
\hline KYOS033 & 37.4098 & 83.8005 & Owsley & 12 & 3.7 & 5.9 & 5.9 & 0.85 & 0.25 & 0.11 & 0.055 \\
\hline KYOS035 & 37.3704 & 83.7927 & Owsley & 7.9 & 4.6 & 0.062 & 3.6 & 1.2 & 0.28 & 0.20 & 0.035 \\
\hline KYOS036 & 37.3678 & 83.7394 & Owsley & 11 & 3.2 & 1.1 & 5.2 & 0.80 & 0.23 & 0.16 & 0.045 \\
\hline KYOS039 & 37.4962 & 83.6928 & Owsley & 13 & 6.1 & 0.71 & 5.3 & 1.6 & 0.45 & 0.22 & 0.055 \\
\hline KYOS040 & 37.4380 & 83.6844 & Owsley & 15 & 7.4 & 0.13 & 5.0 & 2.0 & 0.60 & 0.35 & 0.060 \\
\hline KYOS041 & 37.3610 & 83.6959 & Owsley & 6.2 & 4.0 & 0.095 & 2.7 & 1.1 & 0.28 & 0.31 & 0.050 \\
\hline KYOS042 & 37.3560 & 83.7179 & Owsley & 5.3 & 2.7 & 0.034 & 2.2 & 0.77 & 0.15 & 0.16 & 0.020 \\
\hline KYOS043 & 37.3572 & 83.7692 & Owsley & 9.9 & 5.7 & 0.22 & 4.1 & 1.5 & 0.39 & 0.24 & 0.050 \\
\hline KYOS046 & 37.4865 & 83.8591 & Owsley & 8.1 & 1.8 & 0.40 & 2.9 & 0.34 & 0.083 & 0.020 & 0.020 \\
\hline KYPR001 & 37.3447 & 83.4840 & Perry & 7.4 & 3.8 & 0.078 & 2.7 & 1.1 & 0.27 & 0.17 & 0.030 \\
\hline KYPR002 & 37.3236 & 83.5311 & Perry & 2.2 & 1.5 & 0.022 & 1.2 & 0.42 & 0.077 & 0.055 & 0.015 \\
\hline KYPR003 & 37.2968 & 83.5360 & Perry & 4.4 & 1.5 & 0.017 & 0.94 & 0.51 & 0.072 & 0.050 & 0.010 \\
\hline KYPR004 & 37.3080 & 83.4975 & Perry & 3.5 & 3.1 & 0.12 & 2.3 & 0.92 & 0.28 & 0.27 & 0.030 \\
\hline KYPR005 & 37.3412 & 83.4434 & Perry & 7.8 & 4.7 & 1.6 & 3.0 & 1.4 & 0.35 & 0.30 & 0.035 \\
\hline KYPR006 & 37.3628 & 83.4620 & Perry & 5.2 & 5.2 & 0.11 & 2.6 & 1.5 & 0.37 & 0.26 & 0.030 \\
\hline KYPR007 & 37.2796 & 83.5055 & Perry & 1.2 & 0.84 & 0.017 & 0.50 & 0.29 & 0.039 & 0.035 & 0.005 \\
\hline KYPR008 & 37.2629 & 83.4913 & Perry & 1.1 & 0.87 & 0.017 & 0.42 & 0.35 & 0.033 & 0.035 & 0.005 \\
\hline KYPR009 & 37.3032 & 83.4418 & Perry & 2.4 & 2.5 & 0.062 & 1.3 & 0.76 & 0.15 & 0.15 & 0.020 \\
\hline
\end{tabular}




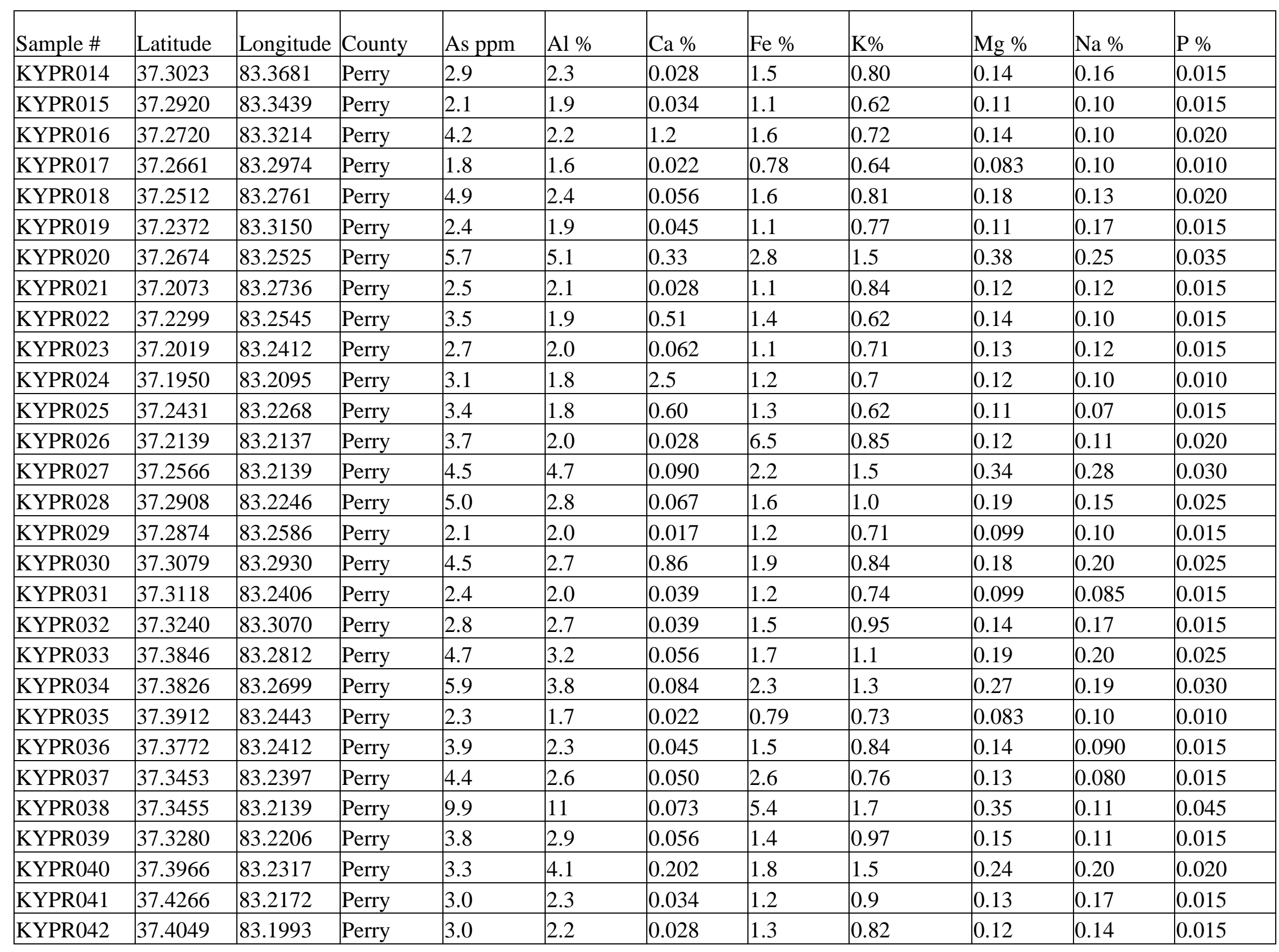




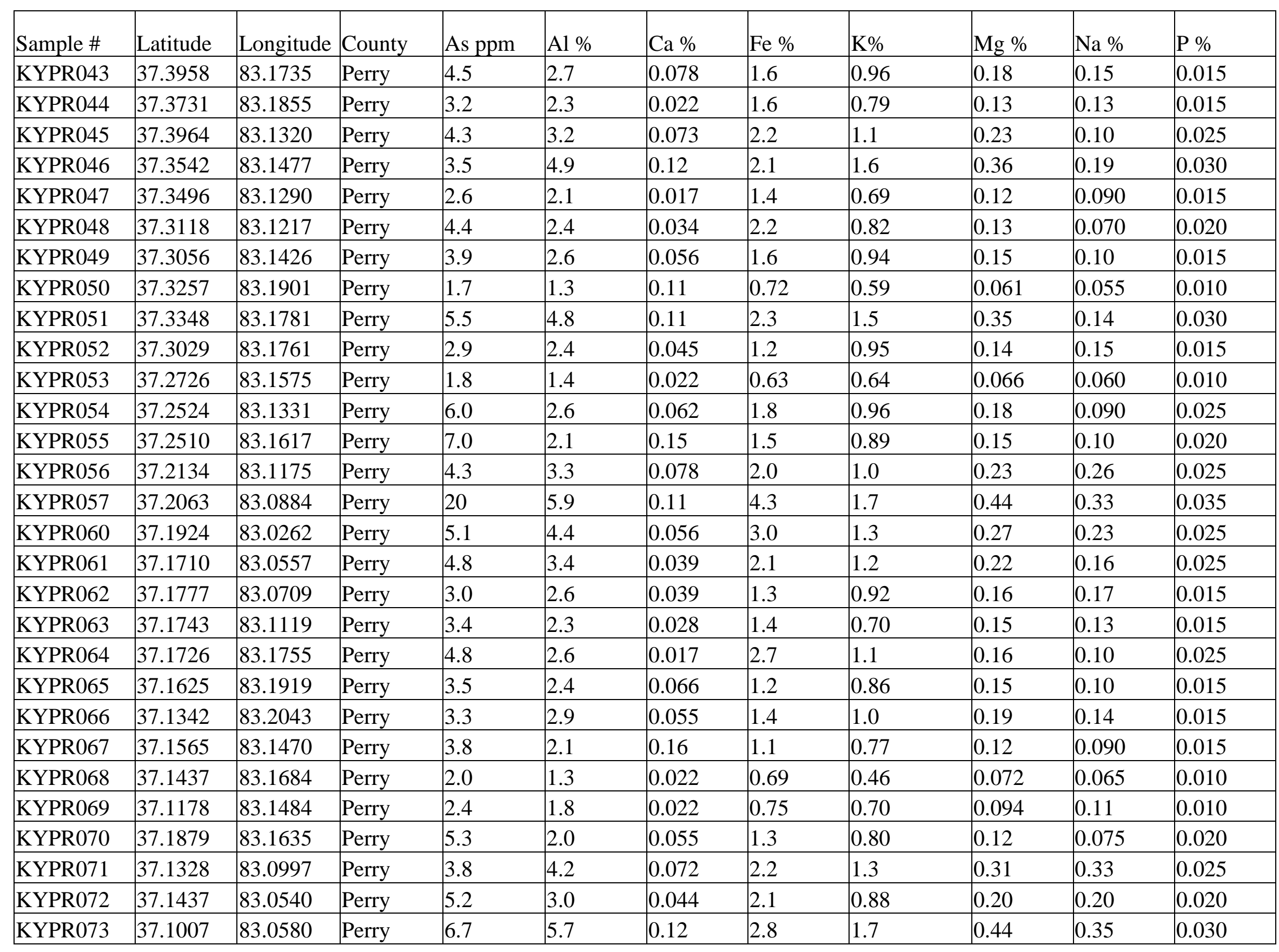




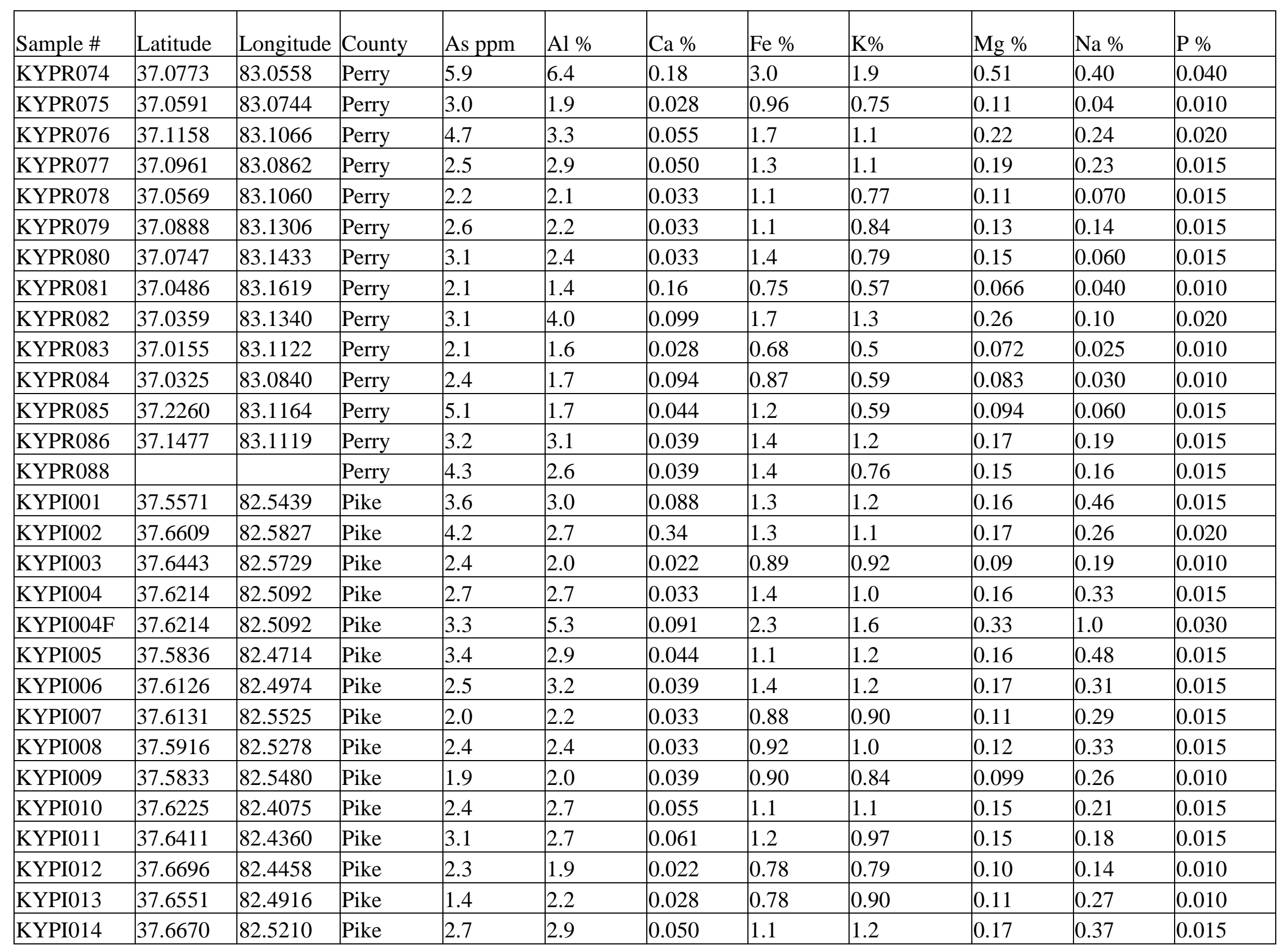




\begin{tabular}{|c|c|c|c|c|c|c|c|c|c|c|c|}
\hline KYPI016 & 37.6287 & 82.3447 & Pike & 3.2 & 2.7 & 0.039 & 1.1 & 1.0 & 0.15 & 0.31 & 0.015 \\
\hline KYPI018 & 37.7204 & 82.3395 & Pike & 10 & 3.2 & 1.4 & 1.5 & 1.1 & 0.24 & 0.34 & 0.015 \\
\hline KYPI018F & 37.7204 & 82.3395 & Pike & 11 & 5.0 & 1.4 & 2.5 & 1.5 & 0.40 & 0.78 & 0.025 \\
\hline KYPI020 & 37.6488 & 82.3311 & Pike & 3.6 & 3.0 & 0.050 & 1.4 & 1.1 & 0.17 & 0.21 & 0.015 \\
\hline KYPI021 & 37.6743 & 82.3416 & Pike & 3.7 & 3.3 & 0.055 & 1.3 & 1.1 & 0.20 & 0.45 & 0.020 \\
\hline KYPI022 & 37.6102 & 82.3765 & Pike & 2.4 & 2.3 & 0.072 & 0.89 & 0.96 & 0.12 & 0.19 & 0.015 \\
\hline KYPI023 & 37.5446 & 82.4422 & Pike & 2.9 & 2.8 & 0.044 & 1.2 & 1.0 & 0.16 & 0.42 & 0.015 \\
\hline KYPI024 & 37.4834 & 82.3538 & Pike & 3.3 & 3.1 & 0.044 & 1.4 & 1.0 & 0.19 & 0.37 & 0.015 \\
\hline KYPI067 & 37.5458 & 82.5745 & Pike & 3.0 & 2.1 & 0.044 & 1.0 & 0.82 & 0.12 & 0.29 & 0.015 \\
\hline KYPI068 & 37.5433 & 82.5206 & Pike & 3.1 & 2.6 & 0.33 & 1.4 & 0.96 & 0.20 & 0.35 & 0.015 \\
\hline KYPI069 & 37.5200 & 82.5537 & Pike & 5.6 & 4.3 & 0.099 & 2.6 & 1.4 & 0.33 & 0.46 & 0.030 \\
\hline KYPI070 & 37.4603 & 82.5111 & Pike & 3.2 & 2.7 & 0.64 & 1.6 & 0.90 & 0.22 & 0.29 & 0.020 \\
\hline KYPI071 & 37.5148 & 82.4798 & Pike & 4.2 & 2.7 & 0.79 & 1.4 & 0.99 & 0.18 & 0.35 & 0.015 \\
\hline KYPI072 & 37.4822 & 82.4628 & Pike & 2.0 & 2.9 & 0.22 & 1.1 & 1.1 & 0.17 & 0.46 & 0.015 \\
\hline KYPI073 & 37.5278 & 82.4852 & Pike & 2.4 & 2.8 & 0.24 & 1.3 & 1.0 & 0.16 & 0.43 & 0.015 \\
\hline KYPI074 & 37.4892 & 82.4156 & Pike & 2.9 & 2.8 & 0.15 & 1.2 & 1.0 & 0.17 & 0.45 & 0.015 \\
\hline KYPI075 & 37.4498 & 82.4114 & Pike & 4.0 & 3.1 & 0.14 & 1.7 & 1.1 & 0.20 & 0.39 & 0.020 \\
\hline KYPI076 & 37.4998 & 82.4354 & Pike & 3.2 & 3.2 & 0.061 & 1.5 & 1.1 & 0.19 & 0.45 & 0.020 \\
\hline
\end{tabular}




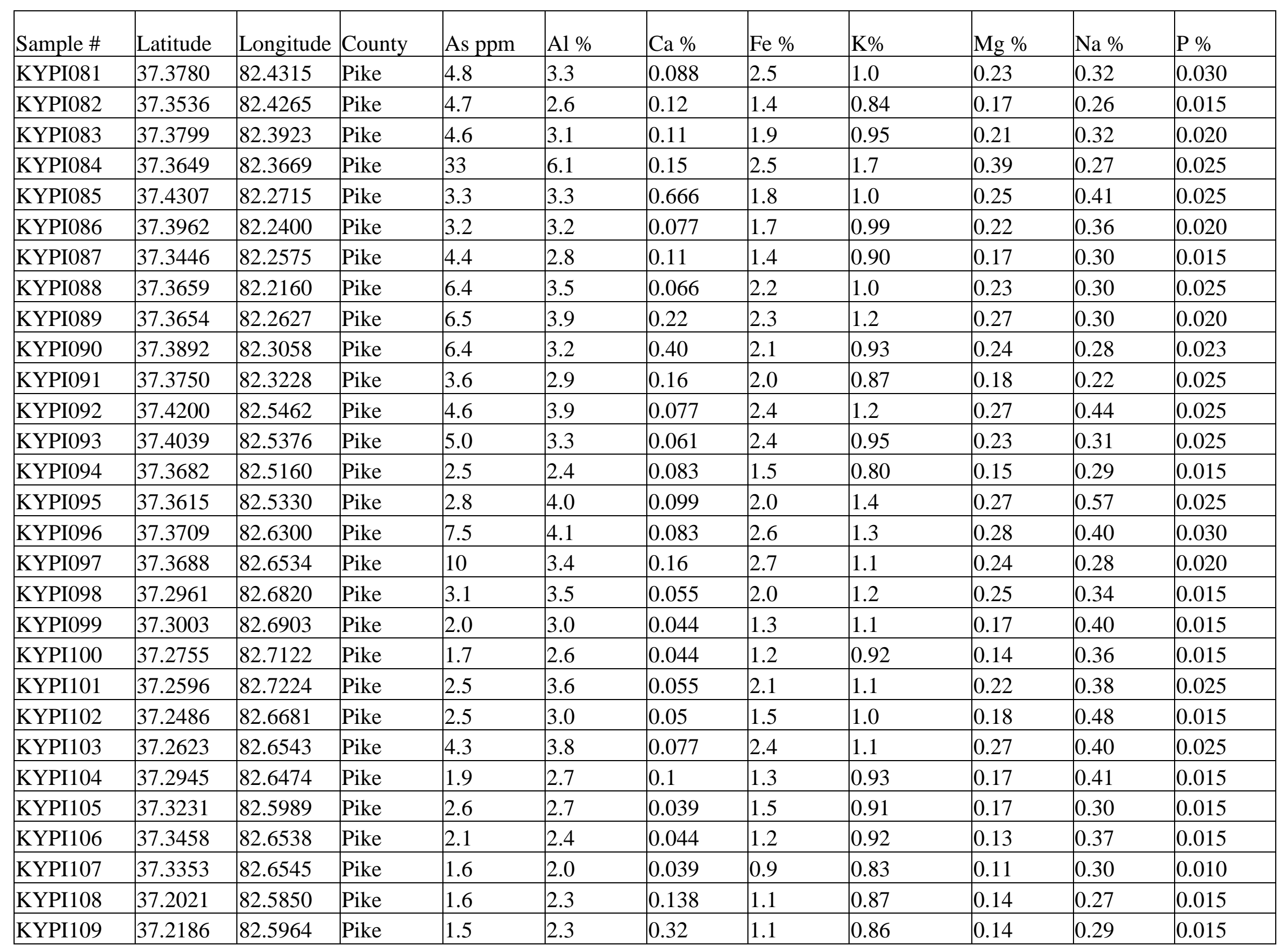




\begin{tabular}{|c|c|c|c|c|c|c|c|c|c|c|c|}
\hline KYPI111 & 37.2571 & 82.5519 & Pike & 2.2 & 2.8 & 0.1 & 1.4 & 0.99 & 0.15 & 0.40 & 0.020 \\
\hline KYPI113 & 37.2754 & 82.6163 & Pike & 4.2 & 3.3 & 0.066 & 1.6 & 1.1 & 0.22 & 0.47 & 0.020 \\
\hline KYPI114 & 37.3992 & 82.5914 & Pike & 12 & 3.9 & 0.1 & 2.5 & 1.2 & 0.26 & 0.28 & 0.020 \\
\hline KYPI117 & 37.3059 & 82.5826 & Pike & 2.4 & 2.9 & 0.072 & 1.5 & 0.99 & 0.19 & 0.31 & 0.020 \\
\hline KYPI118 & 37.3817 & 82.5562 & Pike & 3.2 & 3.4 & 0.099 & 2.2 & 1.1 & 0.23 & 0.43 & 0.020 \\
\hline KYPI119 & 37.3478 & 82.5698 & Pike & 4.8 & 3.6 & 0.12 & 3.9 & 1.1 & 0.26 & 0.30 & 0.030 \\
\hline KYPI120 & 37.3193 & 82.5692 & Pike & 1.9 & 2.0 & 0.044 & 1.1 & 0.70 & 0.12 & 0.23 & 0.015 \\
\hline KYPI121 & 37.3947 & 82.4806 & Pike & 3.0 & 3.9 & 0.061 & 2.2 & 1.2 & 0.26 & 0.43 & 0.025 \\
\hline KYPI125 & 37.3460 & 82.3574 & Pike & 4.1 & 3.7 & 3.7 & 1.9 & 1.1 & 0.37 & 0.42 & 0.030 \\
\hline KYPI126 & 37.3202 & 82.4087 & Pike & 2.4 & 2.4 & 0.14 & 1.3 & 0.80 & 0.15 & 0.26 & 0.015 \\
\hline KYPI127 & 37.2952 & 82.4153 & Pike & 3.0 & 3.1 & 0.061 & 1.5 & 1.0 & 0.20 & 0.28 & 0.015 \\
\hline KYPI128 & 37.6819 & 82.4958 & Pike & 2.4 & 2.7 & 0.1 & 1.2 & 1.1 & 0.15 & 0.31 & 0.015 \\
\hline KYPI129 & 37.6828 & 82.5412 & Pike & 1.4 & 2.4 & 0.033 & 0.9 & 1.0 & 0.12 & 0.25 & 0.010 \\
\hline KYPI130 & 37.6641 & 82.5446 & Pike & 1.9 & 1.5 & 0.039 & 0.8 & 0.63 & 0.08 & 0.16 & 0.010 \\
\hline KYPI131 & 37.5776 & 82.3033 & Pike & 2.6 & 3.1 & 0.072 & 1.3 & 1.0 & 0.18 & 0.46 & 0.015 \\
\hline KYPI132 & 37.5853 & 82.3447 & Pike & 2.5 & 3.1 & 0.055 & 1.4 & 1.2 & 0.17 & 0.43 & 0.020 \\
\hline KYPI133 & 37.5246 & 82.2931 & Pike & 2.7 & 3.0 & 0.16 & 1.3 & 1.1 & 0.17 & 0.37 & 0.015 \\
\hline KYPI134 & 37.5384 & 82.2666 & Pike & 2.8 & 2.8 & 0.044 & 1.2 & 0.96 & 0.16 & 0.40 & 0.015 \\
\hline
\end{tabular}




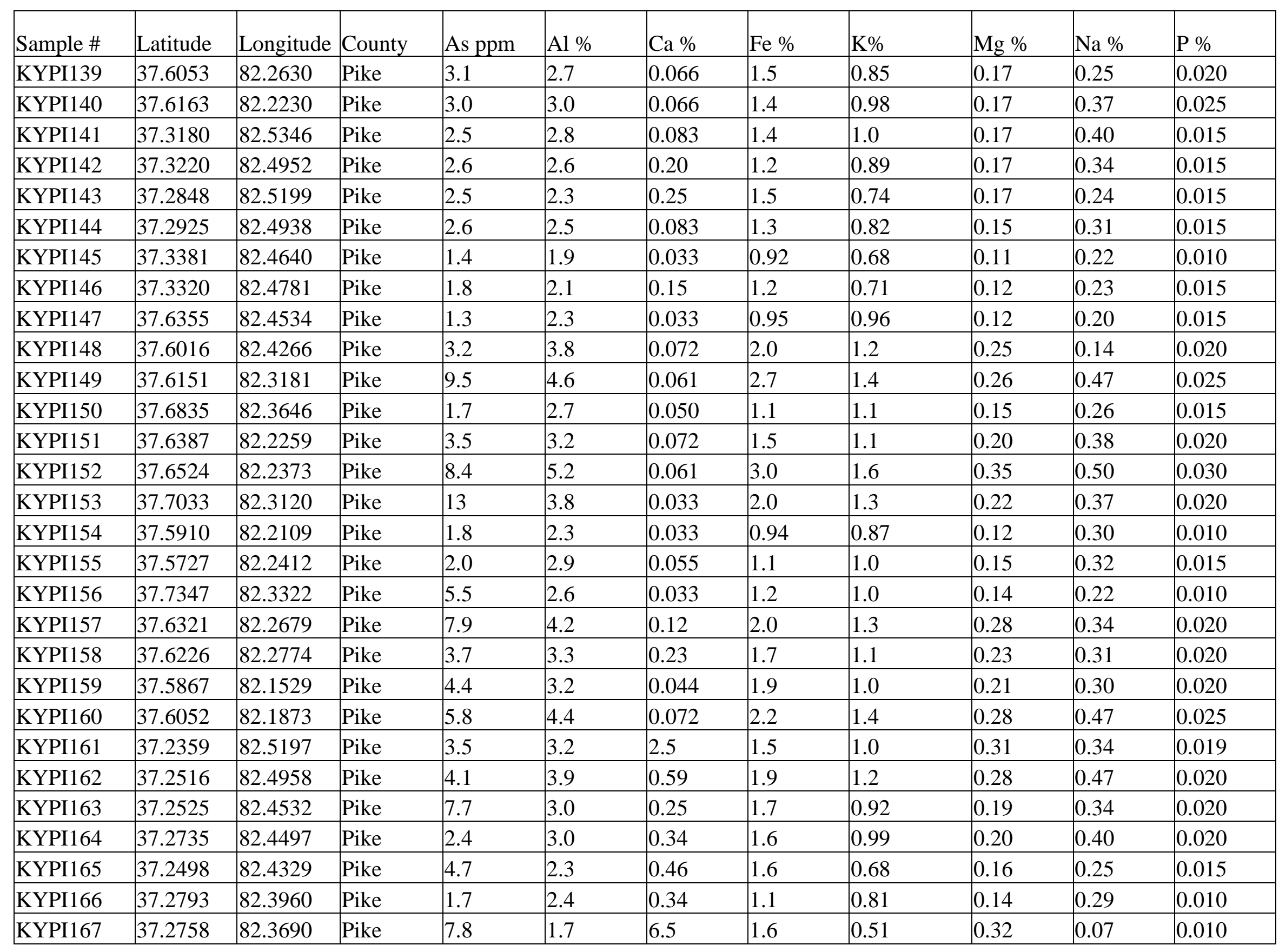




\begin{tabular}{|c|c|c|c|c|c|c|c|c|c|c|c|}
\hline KYPI169 & 37.4236 & 82.5188 & Pike & 4.4 & 3.6 & 0.068 & 2.1 & 1.2 & 0.23 & 0.34 & 0.025 \\
\hline KYPI171 & 37.4581 & 82.3864 & Pike & 3.3 & 3.5 & 0.47 & 1.8 & 1.1 & 0.24 & 0.50 & 0.020 \\
\hline KYPI172 & 37.5669 & 82.1551 & Pike & 7.0 & 5.5 & 0.046 & 2.7 & 1.6 & 0.34 & 0.47 & 0.025 \\
\hline KYPI175 & 37.4438 & 82.5444 & Pike & 1.6 & 2.4 & 0.086 & 1.0 & 0.97 & 0.12 & 0.27 & 0.015 \\
\hline KYPI176 & 37.5066 & 82.5212 & Pike & 4.6 & 3.7 & 0.10 & 2.0 & 1.1 & 0.24 & 0.43 & 0.025 \\
\hline KYPO001 & 37.8438 & 83.6960 & Powell & 2.0 & 1.0 & 0.034 & 0.7 & 0.29 & 0.072 & 0.06 & 0.010 \\
\hline KYPO002 & 37.8570 & 83.7331 & Powell & 5.8 & 1.6 & 0.057 & 1.6 & 0.44 & 0.12 & 0.13 & 0.010 \\
\hline KYPO004 & 37.8812 & 83.7915 & Powell & 16 & 2.9 & 0.086 & 2.4 & 0.89 & 0.28 & 0.21 & 0.015 \\
\hline KYPO008 & 37.8904 & 83.8611 & Powell & 28 & 5.6 & 0.40 & 3.8 & 1.8 & 0.56 & 0.32 & 0.020 \\
\hline KYPO009 & 37.8858 & 83.8833 & Powell & 57 & 7.0 & 1.2 & 5.8 & 2.2 & 0.66 & 0.36 & 0.035 \\
\hline KYPO010 & 37.8646 & 83.8927 & Powell & 80 & 6.7 & 0.13 & 7.0 & 2.4 & 0.56 & 0.27 & 0.060 \\
\hline KYPO011 & 37.8999 & 83.9239 & Powell & 9.4 & 2.0 & 0.33 & 1.5 & 0.60 & 0.17 & 0.15 & 0.010 \\
\hline KYPO011F & 37.8999 & 83.9239 & Powell & 9.4 & 3.6 & 0.22 & 1.9 & 1.2 & 0.31 & 0.38 & 0.015 \\
\hline KYPO012 & 37.8565 & 83.9799 & Powell & 89 & 7.0 & 1.0 & 10.2 & 2.1 & 0.82 & 0.12 & 0.13 \\
\hline KYPO013 & 37.8851 & 83.9874 & Powell & 56 & 7.8 & 0.33 & 6.9 & 2.3 & 0.57 & 0.14 & 0.090 \\
\hline KYPO014 & 37.9172 & 83.9725 & Powell & 56 & 6.4 & 0.029 & 8.9 & 2.0 & 0.42 & 0.14 & 0.10 \\
\hline KYPO015 & 37.9113 & 83.9439 & Powell & 42 & 3.9 & 0.051 & 3.3 & 1.3 & 0.37 & 0.25 & 0.015 \\
\hline KYPO016 & 37.8265 & 83.9330 & Powell & 98 & 6.6 & 0.04 & 10 & 1.9 & 0.43 & 0.12 & 0.15 \\
\hline
\end{tabular}

60 


\begin{tabular}{|c|c|c|c|c|c|c|c|c|c|c|c|}
\hline KYPO020F & 37.8029 & 83.8611 & Powell & 8.6 & 5.4 & 0.41 & 2.8 & 1.8 & 0.52 & 0.44 & 0.020 \\
\hline KYPO022 & 37.8310 & 83.8166 & Powell & 40 & 8.0 & 0.057 & 5.4 & 2.6 & 0.72 & 0.39 & 0.030 \\
\hline KYPO023 & 37.7965 & 83.7978 & Powell & 2.2 & 0.91 & 0.28 & 0.75 & 0.21 & 0.072 & 0.05 & 0.005 \\
\hline KYPO025 & 37.7686 & 83.7804 & Powell & 0.8 & 0.46 & 0.029 & 0.22 & 0.15 & 0.022 & 0.02 & 0.005 \\
\hline KYPO026 & 37.8168 & 83.7741 & Powell & 3.3 & 1.6 & 18 & 1.1 & 0.51 & 0.25 & 0.11 & 0.015 \\
\hline KYPO026F & 37.8168 & 83.7741 & Powell & 5.1 & 3.1 & 16 & 1.9 & 1.1 & 0.40 & 0.24 & 0.020 \\
\hline KYPO027 & 37.8068 & 83.7666 & Powell & 1.8 & 1.2 & 0.37 & 1.0 & 0.3 & 0.099 & 0.07 & 0.010 \\
\hline KYPO028 & 37.8310 & 83.7278 & Powell & 8.7 & 2.7 & 0.43 & 2.0 & 0.77 & 0.23 & 0.20 & 0.015 \\
\hline KYWH001 & 36.7496 & 84.2058 & Whitley & 5.7 & 5.8 & 0.051 & 2.4 & 1.3 & 0.28 & 0.13 & 0.020 \\
\hline KYWH002 & 36.7346 & 84.2224 & Whitley & 2.6 & 2.1 & 0.063 & 1.4 & 0.50 & 0.099 & 0.14 & 0.020 \\
\hline KYWH003 & 36.7046 & 84.2824 & Whitley & 4.0 & 2.4 & 0.040 & 1.9 & 0.60 & 0.12 & 0.18 & 0.025 \\
\hline KYWH004 & 36.7187 & 84.1743 & Whitley & 5.2 & 5.2 & 0.068 & 2.2 & 1.3 & 0.31 & 0.11 & 0.025 \\
\hline KYWH005 & 36.7429 & 84.2829 & Whitley & 1.0 & 1.2 & 0.023 & 0.51 & 0.30 & 0.1 & 0.074 & 0.005 \\
\hline KYWH006 & 36.6856 & 84.2312 & Whitley & 5.1 & 4.2 & 0.086 & 2.3 & 1.3 & 0.28 & 0.32 & 0.030 \\
\hline KYWH007 & 36.6204 & 84.2312 & Whitley & 5.2 & 5.7 & 0.17 & 2.7 & 1.5 & 0.41 & 0.25 & 0.040 \\
\hline KYWH008 & 36.6476 & 84.2395 & Whitley & 3.9 & 2.7 & 0.04 & 1.8 & 0.68 & 0.14 & 0.16 & 0.020 \\
\hline KYWH009 & 36.5957 & 84.1662 & Whitley & 7.0 & 6.3 & 0.19 & 3.4 & 1.8 & 0.53 & 0.28 & 0.050 \\
\hline KYWH010 & 36.6136 & 84.1431 & Whitley & 6.4 & 7.7 & 0.25 & 3.4 & 2.1 & 0.56 & 0.29 & 0.050 \\
\hline
\end{tabular}




\begin{tabular}{|c|c|c|c|c|c|c|c|c|c|c|c|}
\hline KYWH016 & 36.6767 & 83.9053 & Whitley & 4.2 & 4.8 & 0.103 & 2.6 & 1.3 & 0.31 & 0.27 & 0.035 \\
\hline KYWH018 & 36.7112 & 83.9586 & Whitley & 4.0 & 4.6 & 0.13 & 2.5 & 1.3 & 0.28 & 0.26 & 0.035 \\
\hline KYWH019 & 36.6251 & 84.0376 & Whitley & 4.0 & 3.6 & 0.11 & 2.2 & 1.0 & 0.23 & 0.23 & 0.030 \\
\hline KYWH022 & 36.7144 & 84.0171 & Whitley & 3.1 & 3.1 & 0.051 & 1.5 & 0.92 & 0.16 & 0.31 & 0.020 \\
\hline KYWH023 & 36.7170 & 84.1268 & Whitley & 7.6 & 8.8 & 0.14 & 3.8 & 2.4 & 0.63 & 0.30 & 0.060 \\
\hline KYWH024 & 36.7439 & 84.1044 & Whitley & 5.9 & 7.2 & 0.14 & 3.3 & 2.0 & 0.52 & 0.36 & 0.050 \\
\hline KYWH025 & 36.7587 & 84.0610 & Whitley & 4.4 & 4.6 & 0.11 & 2.5 & 1.2 & 0.26 & 0.24 & 0.035 \\
\hline KYWH026 & 36.7761 & 83.9911 & Whitley & 3.6 & 3.5 & 0.063 & 1.7 & 0.91 & 0.18 & 0.19 & 0.025 \\
\hline KYWH030 & 36.8421 & 84.0542 & Whitley & 4.5 & 4.9 & 0.11 & 2.6 & 1.3 & 0.30 & 0.42 & 0.040 \\
\hline KYWH031 & 36.8319 & 84.1154 & Whitley & 4.4 & 3.7 & 0.068 & 2.1 & 1.1 & 0.21 & 0.42 & 0.025 \\
\hline KYWH032 & 36.8010 & 84.1399 & Whitley & 5.7 & 4.3 & 0.19 & 2.5 & 1.0 & 0.26 & 0.16 & 0.035 \\
\hline KYWH033 & 36.7891 & 84.1903 & Whitley & 4.3 & 3.7 & 0.091 & 2.0 & 0.86 & 0.23 & 0.14 & 0.030 \\
\hline KYWH034 & 36.8288 & 84.2220 & Whitley & 2.4 & 2.8 & 0.057 & 1.3 & 0.68 & 0.13 & 0.13 & 0.020 \\
\hline KYWH035 & 36.8519 & 84.1929 & Whitley & 4.5 & 4.3 & 0.080 & 2.2 & 1.1 & 0.23 & 0.20 & 0.030 \\
\hline KYWH036 & 36.8809 & 84.1869 & Whitley & 3.1 & 3.2 & 0.034 & 1.6 & 0.79 & 0.15 & 0.11 & 0.020 \\
\hline KYWH037 & 36.9133 & 84.1197 & Whitley & 6.4 & 4.6 & 0.182 & 2.7 & 1.1 & 0.30 & 0.16 & 0.050 \\
\hline KYWH038 & 36.9424 & 84.1498 & Whitley & 2.9 & 2.6 & 0.068 & 1.3 & 0.60 & 0.12 & 0.13 & 0.020 \\
\hline KYWH039 & 36.9242 & 84.1743 & Whitley & 1.3 & 1.5 & 0.046 & 0.72 & 0.35 & 0.066 & 0.053 & 0.010 \\
\hline
\end{tabular}




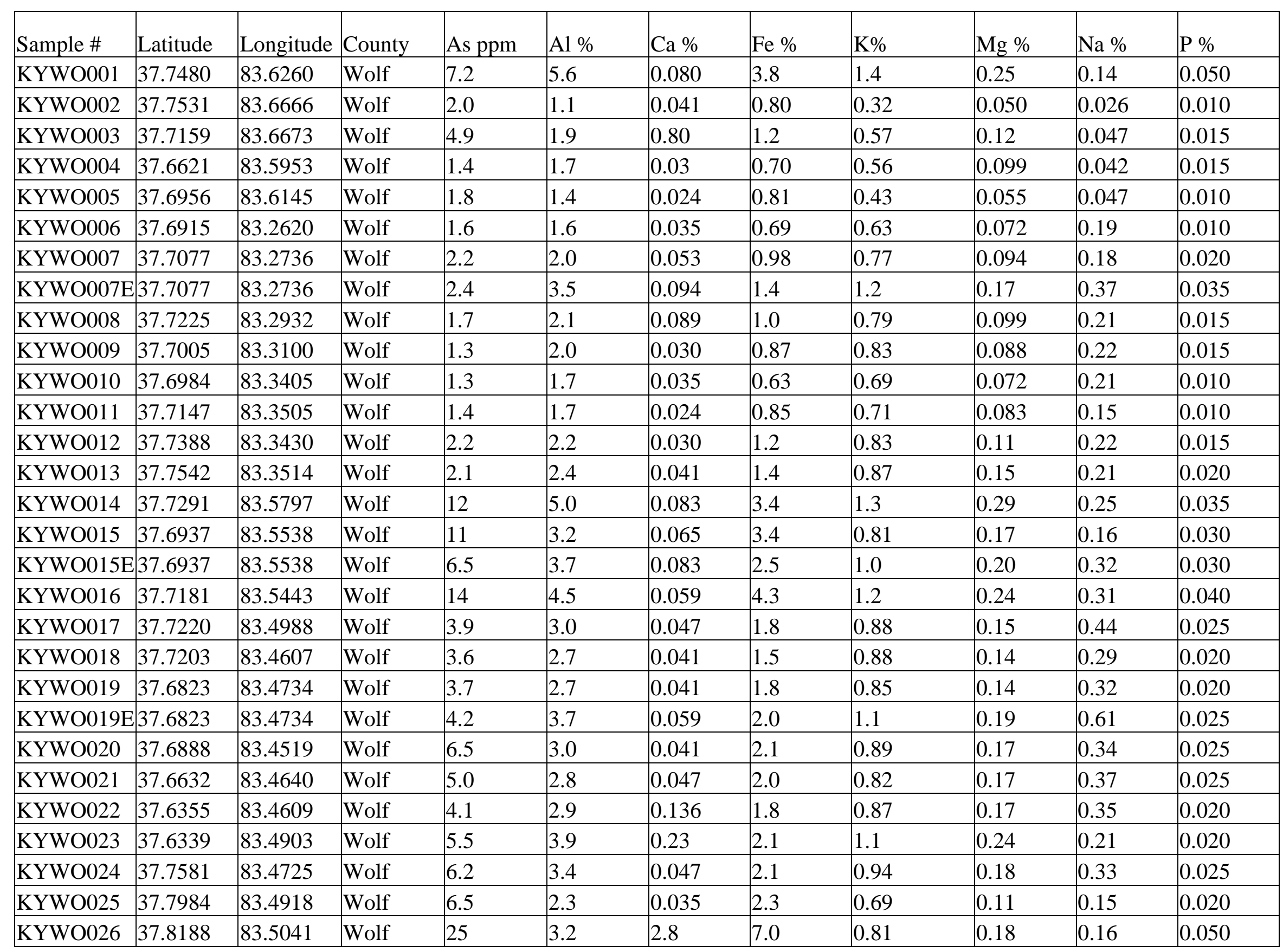




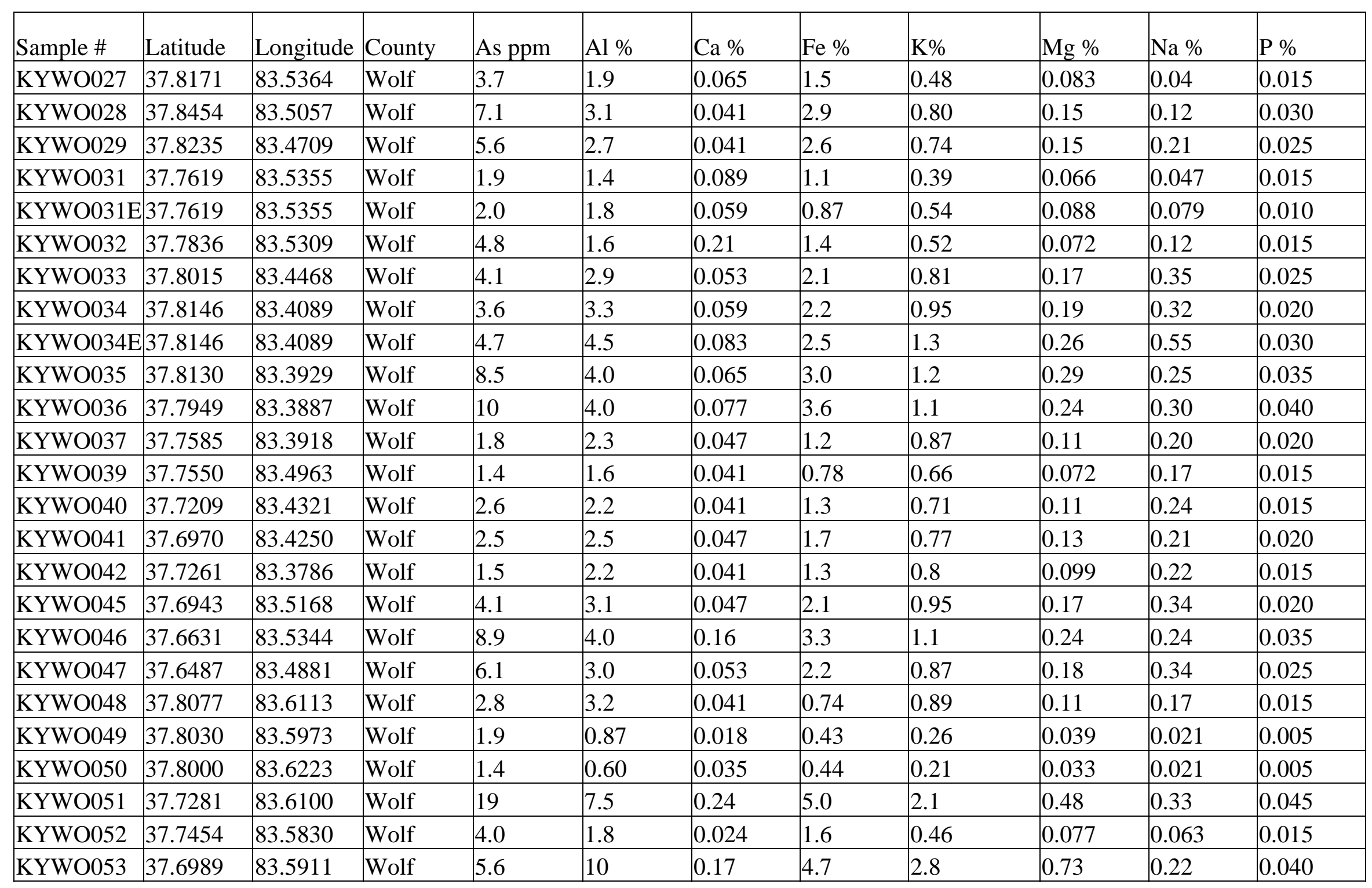




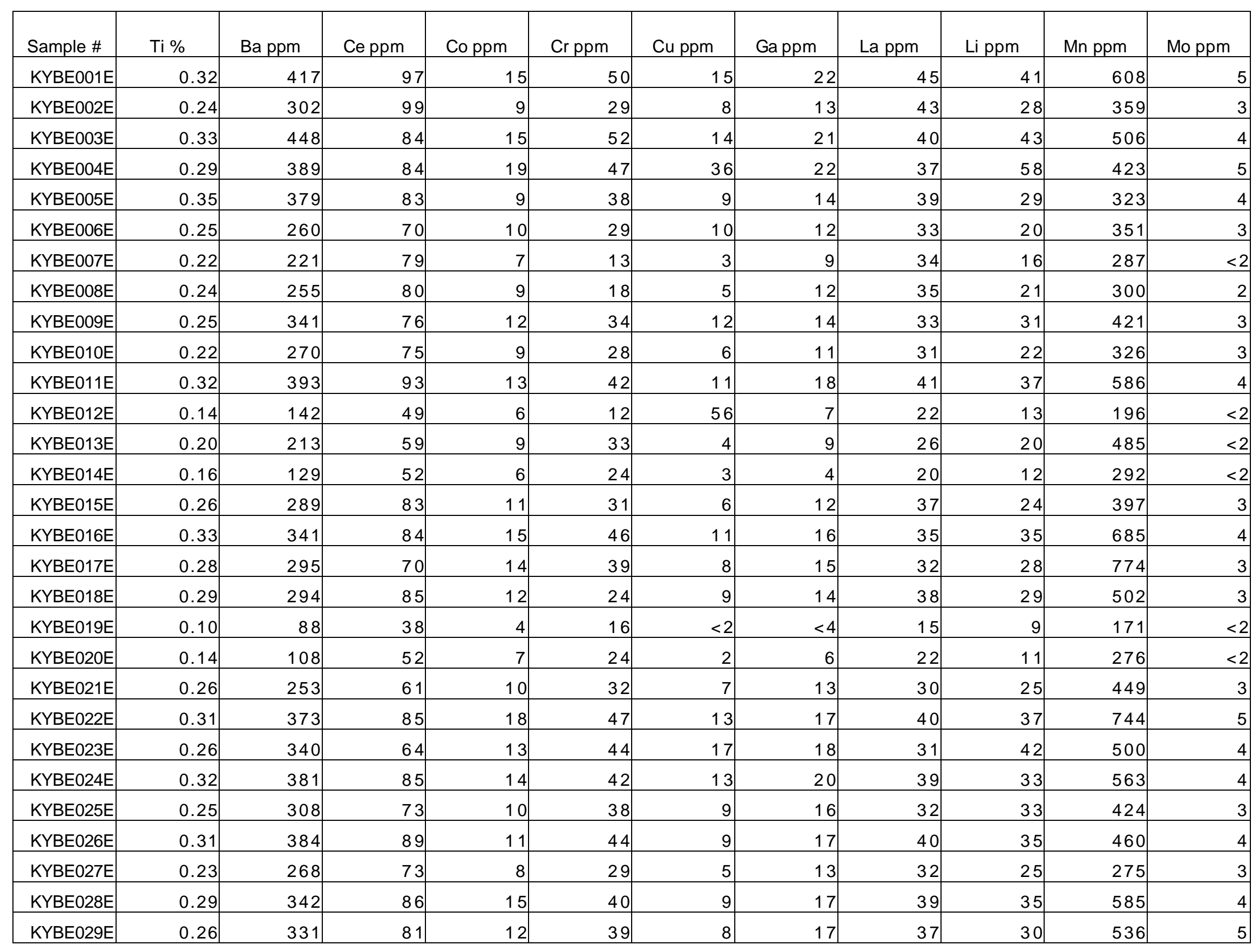




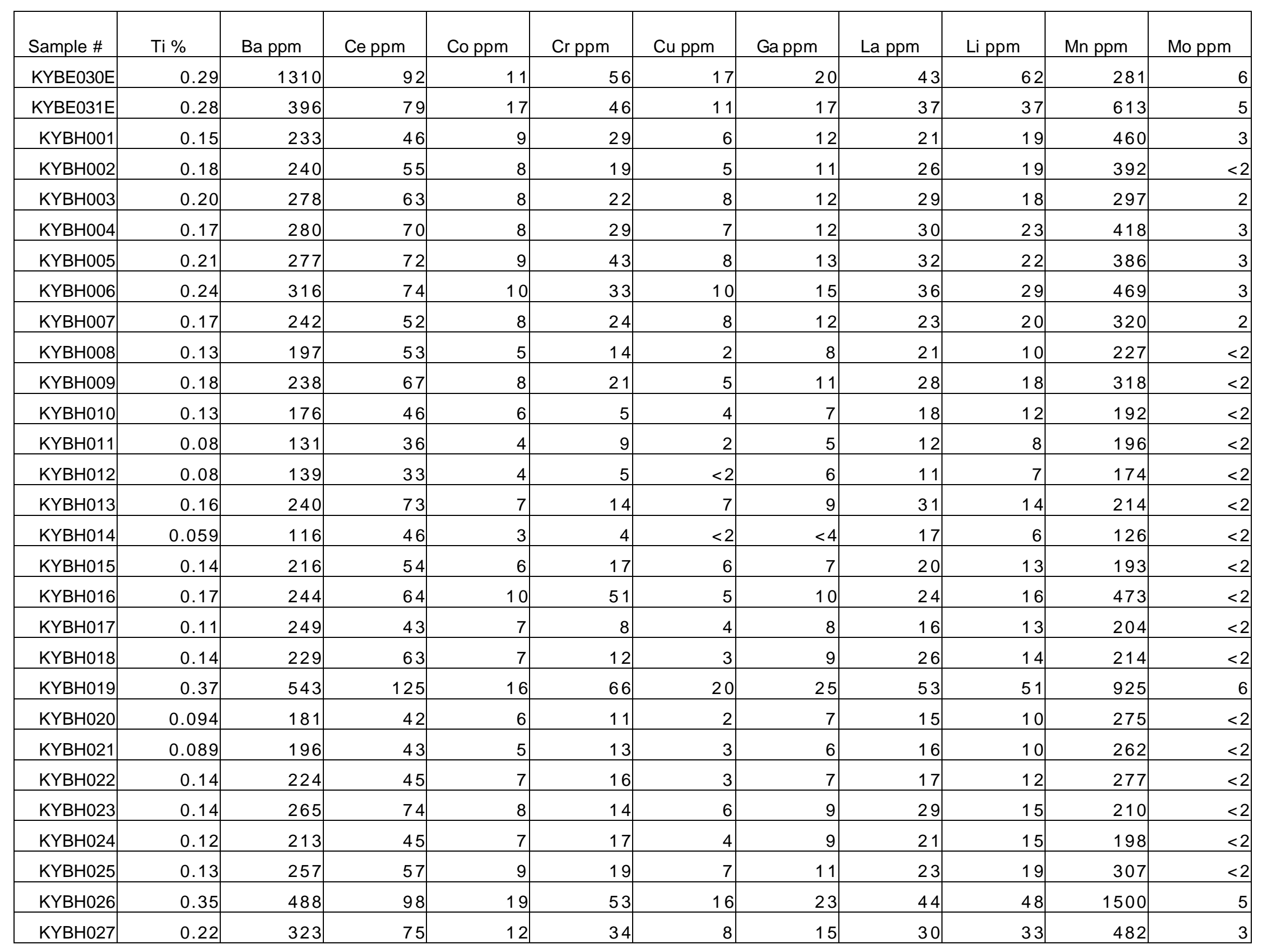




\begin{tabular}{|c|c|c|c|c|c|c|c|c|c|c|c|}
\hline Sample \# & $\mathrm{Ti} \%$ & Ba ppm & Ce ppm & Co ppm & Cr ppm & Cu ppm & Ga ppm & La ppm & Li ppm & Mn ppm & Mo ppm \\
\hline KYBH028 & 0.21 & 327 & 69 & 11 & 30 & 7 & 13 & 28 & 25 & 418 & 3 \\
\hline KYBH029 & 0.083 & 158 & 34 & 5 & 12 & 3 & 5 & 14 & 9 & 231 & $<2$ \\
\hline KYBH031 & 0.27 & 400 & 87 & 13 & 35 & 11 & 17 & 38 & 25 & 503 & 3 \\
\hline KYBH032 & 0.15 & 268 & 49 & 11 & 20 & 6 & 11 & 20 & 15 & 387 & $<2$ \\
\hline $\mathrm{KYBH} 035$ & 0.094 & 175 & 61 & 4 & 4 & 2 & 7 & 23 & 8 & 185 & $<2$ \\
\hline KYBH036 & 0.14 & 228 & 66 & 6 & 5 & 4 & 8 & 26 & 13 & 272 & $<2$ \\
\hline $\mathrm{KYBH} 037$ & 0.059 & 143 & 32 & 3 & $<2$ & $<2$ & $<4$ & 11 & 6 & 133 & $<2$ \\
\hline $\mathrm{KYBH} 038$ & 0.10 & 201 & 48 & 4 & 10 & $<2$ & 6 & 19 & 9 & 113 & $<2$ \\
\hline KYBH039 & 0.19 & 260 & 63 & 13 & 23 & 8 & 10 & 23 & 16 & 489 & $<2$ \\
\hline $\mathrm{KYBH} 043$ & 0.09 & 200 & 40 & 5 & 3 & 2 & 5 & 14 & 8 & 184 & $<2$ \\
\hline $\mathrm{KYBH} 044$ & 0.18 & 228 & 55 & 7 & 7 & 5 & 11 & 21 & 31 & 229 & 2 \\
\hline $\mathrm{KYBH} 045$ & 0.13 & 193 & 44 & 5 & 7 & 3 & 7 & 18 & 11 & 182 & $<2$ \\
\hline KYBH046 & 0.089 & 156 & 40 & 6 & 9 & 3 & 5 & 16 & 8 & 213 & $<2$ \\
\hline $\mathrm{KYBH} 047$ & 0.10 & 192 & 46 & 6 & 11 & 4 & 7 & 16 & 11 & 252 & $<2$ \\
\hline $\mathrm{KYBH} 048$ & 0.10 & 191 & 30 & 6 & 6 & 3 & 8 & 13 & 9 & 191 & $<2$ \\
\hline KYBH049 & 0.11 & 182 & 40 & 12 & 10 & 5 & 8 & 15 & 12 & 423 & $<2$ \\
\hline $\mathrm{KYBH} 050$ & 0.089 & 155 & 32 & 6 & 2 & 3 & $<4$ & 12 & 8 & 457 & $<2$ \\
\hline KYBH051 & 0.077 & 177 & 42 & 5 & 5 & 2 & 6 & 14 & 8 & 137 & $<2$ \\
\hline KYBH052 & 0.12 & 222 & 40 & 7 & 5 & 3 & 7 & 14 & 12 & 233 & $<2$ \\
\hline
\end{tabular}




\begin{tabular}{|c|c|c|c|c|c|c|c|c|c|c|c|}
\hline Sample \# & $\mathrm{Ti} \%$ & Ba ppm & Ce ppm & Co ppm & Cr ppm & Cu ppm & Ga ppm & La ppm & Li ppm & Mn ppm & Mo ppm \\
\hline KYBH058 & 0.083 & 156 & 31 & 3 & $<2$ & $<2$ & 5 & 9 & 7 & 79 & $<2$ \\
\hline KYBH060 & 0.059 & 139 & 33 & 5 & 2 & $<2$ & 4 & 10 & 9 & 276 & $<2$ \\
\hline KYBH061 & 0.11 & 160 & 37 & 11 & 14 & 6 & 8 & 14 & 14 & 425 & $<2$ \\
\hline KYBH064 & 0.18 & 305 & 58 & 9 & 20 & 5 & 10 & 25 & 21 & 338 & $<2$ \\
\hline KYBH065 & 0.12 & 191 & 44 & 5 & 14 & 4 & 8 & 16 & 19 & 161 & $<2$ \\
\hline KYBH066 & 0.11 & 325 & 46 & 5 & 9 & $<2$ & 6 & 17 & 22 & 193 & $<2$ \\
\hline KYBH067 & 0.15 & 243 & 55 & 8 & 10 & 4 & 9 & 21 & 32 & 290 & $<2$ \\
\hline KYBH068 & 0.12 & 235 & 64 & 6 & 3 & 3 & 7 & 26 & 12 & 203 & $<2$ \\
\hline KYBH072 & 0.11 & 184 & 39 & 5 & 6 & 3 & 6 & 13 & 32 & 135 & $<2$ \\
\hline $\mathrm{KYBH} 073$ & 0.15 & 239 & 60 & 7 & 28 & 5 & 10 & 23 & 43 & 281 & $<2$ \\
\hline KYBH074 & 0.11 & 187 & 42 & 6 & 13 & 3 & 7 & 15 & 15 & 203 & $<2$ \\
\hline KYBH075 & 0.14 & 217 & 52 & 9 & 15 & 12 & 11 & 18 & 23 & 287 & $<2$ \\
\hline $\mathrm{KYBH} 076$ & 0.19 & 285 & 56 & 10 & 28 & 12 & 12 & 25 & 28 & 370 & 3 \\
\hline $\mathrm{KYBH} 077 \mathrm{~F}$ & 0.25 & 368 & 85 & 8 & 36 & 9 & 15 & 40 & 27 & 357 & 4 \\
\hline $\mathrm{KYBH} 078 \mathrm{~F}$ & 0.29 & 366 & 99 & 10 & 33 & 8 & 17 & 47 & 29 & 380 & 4 \\
\hline $\mathrm{KYBH} 079 \mathrm{~F}$ & 0.24 & 334 & 95 & 7 & 29 & 7 & 12 & 42 & 22 & 354 & 3 \\
\hline $\mathrm{KYBH} 080 \mathrm{~F}$ & 0.31 & 391 & 104 & 11 & 30 & 12 & 18 & 48 & 33 & 366 & 4 \\
\hline KYBH081F & 0.22 & 315 & 86 & 7 & 17 & 8 & 14 & 38 & 23 & 307 & 4 \\
\hline
\end{tabular}




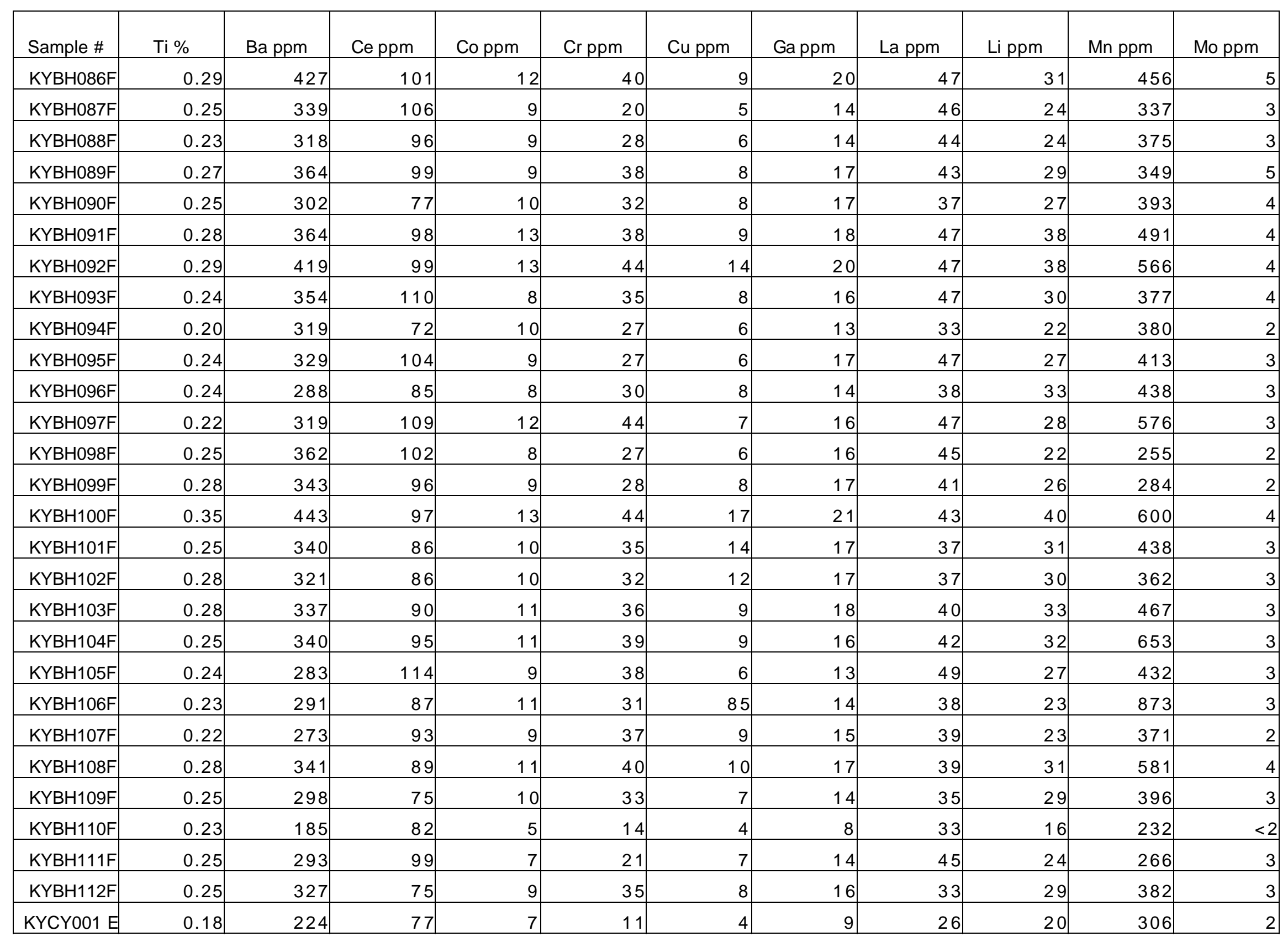




\begin{tabular}{|c|c|c|c|c|c|c|c|c|c|c|c|}
\hline Sample \# & $\mathrm{Ti} \%$ & Ba ppm & Ce ppm & Co ppm & Cr ppm & Cu ppm & Ga ppm & La ppm & Li ppm & Mn ppm & Mo ppm \\
\hline KYCY002 E & 0.21 & 277 & 69 & 10 & 11 & 7 & 12 & 28 & 27 & 416 & 2 \\
\hline KYCY003 E & 0.19 & 248 & 83 & 9 & 33 & 6 & 12 & 32 & 30 & 385 & $<2$ \\
\hline KYCY005 E & 0.21 & 276 & 70 & 9 & 19 & 6 & 12 & 27 & 51 & 403 & 2 \\
\hline KYCY006 E & 0.20 & 293 & 85 & 11 & 17 & 7 & 14 & 34 & 29 & 526 & 3 \\
\hline KYCY009 E & 0.19 & 278 & 74 & 10 & 27 & 8 & 12 & 30 & 31 & 456 & $\underline{2}$ \\
\hline KYCY010 E & 0.27 & 386 & 90 & 14 & 29 & 14 & 17 & 35 & 51 & 538 & 3 \\
\hline KYCY011 E & 0.25 & 372 & 94 & 12 & 22 & 11 & 16 & 36 & 47 & 398 & 3 \\
\hline KYCY012 E & 0.24 & 293 & 90 & 10 & 28 & 6 & 14 & 35 & 51 & 366 & 3 \\
\hline KYCY013 E & 0.25 & 336 & 86 & 11 & 29 & 6 & 13 & 37 & 56 & 393 & 3 \\
\hline KYCY017 E & 0.22 & 301 & 80 & 9 & 15 & 5 & 12 & 33 & 19 & 333 & 2 \\
\hline KYCY018 E & 0.24 & 322 & 135 & 11 & 21 & 6 & 13 & 49 & 21 & 370 & $<2$ \\
\hline KYCY019 E & 0.22 & 291 & 100 & 10 & 25 & 5 & 13 & 39 & 21 & 460 & $<2$ \\
\hline KYCY020 E & 0.24 & 281 & 81 & 14 & 48 & 10 & 15 & 30 & 30 & 468 & 2 \\
\hline KYCY021 E & 0.24 & 277 & 82 & 15 & 36 & 9 & 15 & 29 & 28 & 718 & 2 \\
\hline KYCY022 E & 0.18 & 232 & 72 & 13 & 34 & 9 & 13 & 28 & 24 & 397 & $<2$ \\
\hline KYCY023 E & 0.22 & 224 & 69 & 12 & 23 & 6 & 12 & 24 & 23 & 664 & $<2$ \\
\hline KYCY024 E & 0.21 & 199 & 86 & 8 & 13 & 3 & 8 & 31 & 15 & 444 & $<2$ \\
\hline KYCY025 E & 0.21 & 233 & 79 & 10 & 17 & 5 & 10 & 28 & 19 & 539 & $<2$ \\
\hline KYCY026 E & 0.20 & 248 & 74 & 9 & 19 & 6 & 11 & 27 & 19 & 305 & $<2$ \\
\hline
\end{tabular}




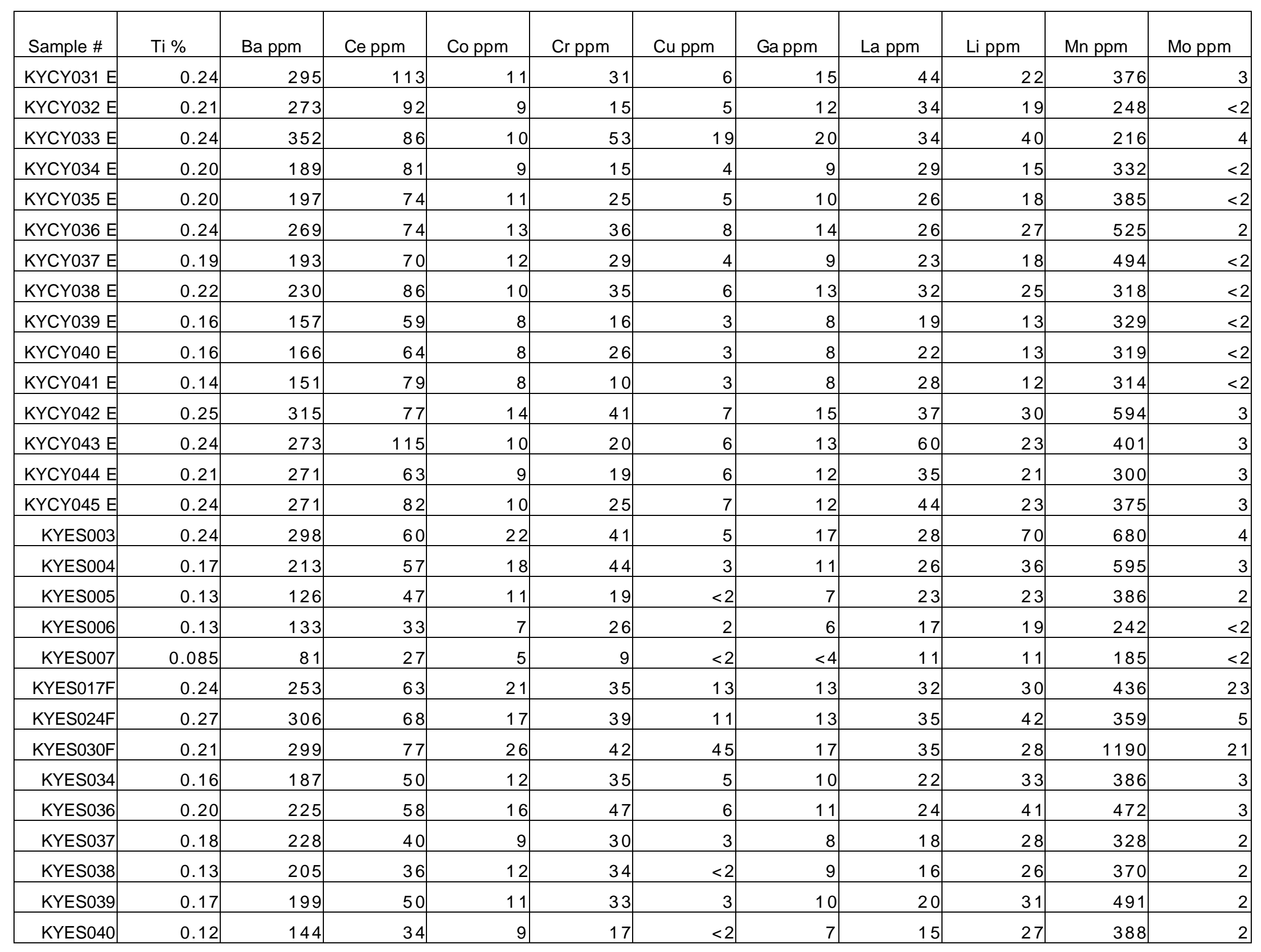




\begin{tabular}{|c|c|c|c|c|c|c|c|c|c|c|c|}
\hline Sample \# & $\mathrm{Ti} \%$ & Ba ppm & Ce ppm & Co ppm & Cr ppm & Cu ppm & Gappm & La ppm & Li ppm & Mn ppm & Mo ppm \\
\hline KYFO001 & 0.10 & 200 & 36 & 6 & 17 & 6 & 9 & 17 & 12 & 245 & 2 \\
\hline KYFO003 & 0.10 & 193 & 34 & 5 & 15 & $<2$ & 8 & 17 & 10 & 219 & $<2$ \\
\hline KYFO004 & 0.13 & 229 & 43 & 7 & 17 & 4 & 10 & 20 & 15 & 281 & $<2$ \\
\hline KYFO007 & 0.12 & 235 & 34 & 6 & 16 & 7 & 9 & 17 & 13 & 235 & $<2$ \\
\hline KYFO008 & 0.17 & 279 & 46 & 9 & 22 & 4 & 11 & 24 & 16 & 358 & 2 \\
\hline KYFO009 & 0.10 & 237 & 31 & 5 & 15 & 4 & 7 & 16 & 10 & 170 & 2 \\
\hline KYFO010 & 0.10 & 185 & 28 & 5 & 8 & 3 & 8 & 13 & 11 & 205 & $<2$ \\
\hline KYFO011 & 0.12 & 246 & 30 & 7 & 28 & 6 & 8 & 18 & 14 & 210 & $<2$ \\
\hline KYFO015 & 0.13 & 232 & 37 & 5 & 8 & 3 & 8 & 19 & 11 & 184 & $<2$ \\
\hline KYFO016 & 0.12 & 224 & 28 & 6 & 5 & 3 & 7 & 16 & 12 & 165 & $<2$ \\
\hline KYFO017 & 0.13 & 289 & 48 & 6 & 19 & 5 & 9 & 20 & 15 & 208 & 2 \\
\hline KYFO018 & 0.13 & 277 & 40 & 6 & 18 & 4 & 10 & 21 & 14 & 228 & 2 \\
\hline KYFO019 & 0.10 & 229 & 29 & 5 & 13 & 2 & 7 & 14 & 9 & 135 & $<2$ \\
\hline KYFO020 & 0.11 & 229 & 32 & 7 & 34 & 6 & 10 & 16 & 12 & 157 & 3 \\
\hline KYFO021 & 0.15 & 273 & 49 & 10 & 23 & 11 & 13 & 21 & 21 & 367 & 3 \\
\hline KYFO022 & 0.13 & 235 & 39 & 8 & 17 & 4 & 9 & 19 & 15 & 286 & 2 \\
\hline KYFO023 & 0.12 & 220 & 40 & 8 & 15 & 6 & 9 & 19 & 15 & 299 & $<2$ \\
\hline KYFO024 & 0.12 & 243 & 45 & 5 & 14 & 7 & 9 & 18 & 14 & 158 & $<2$ \\
\hline
\end{tabular}




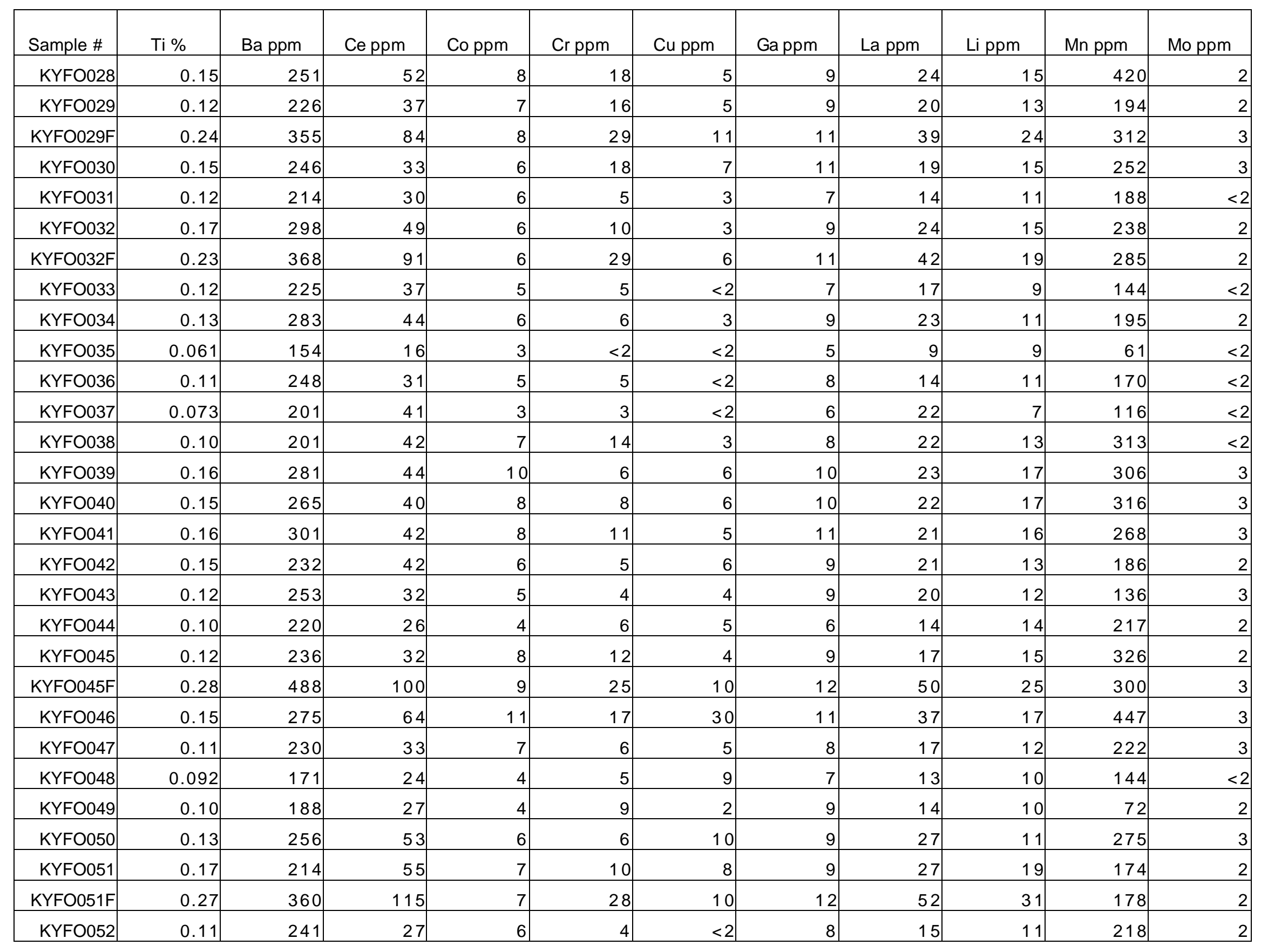




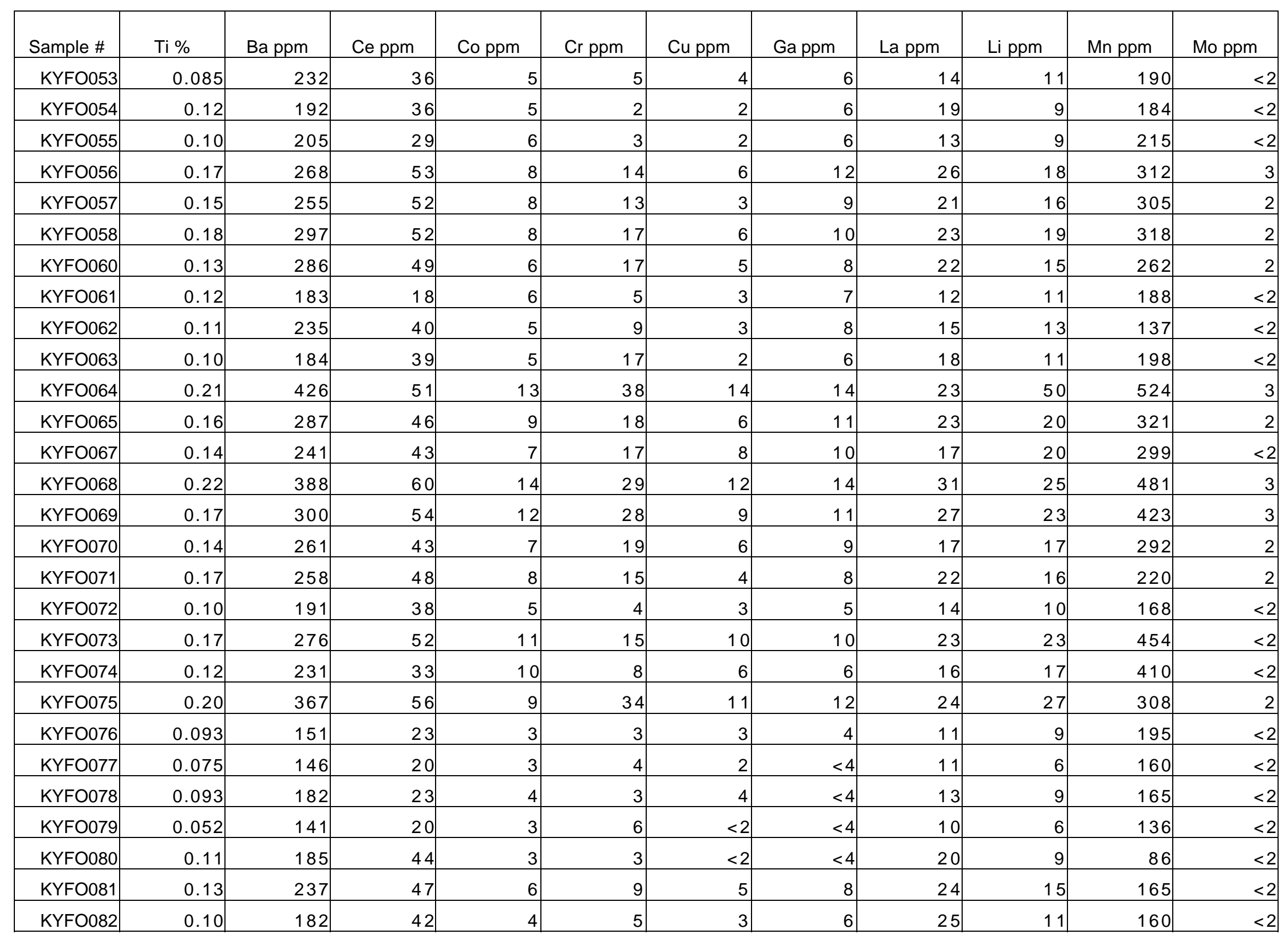




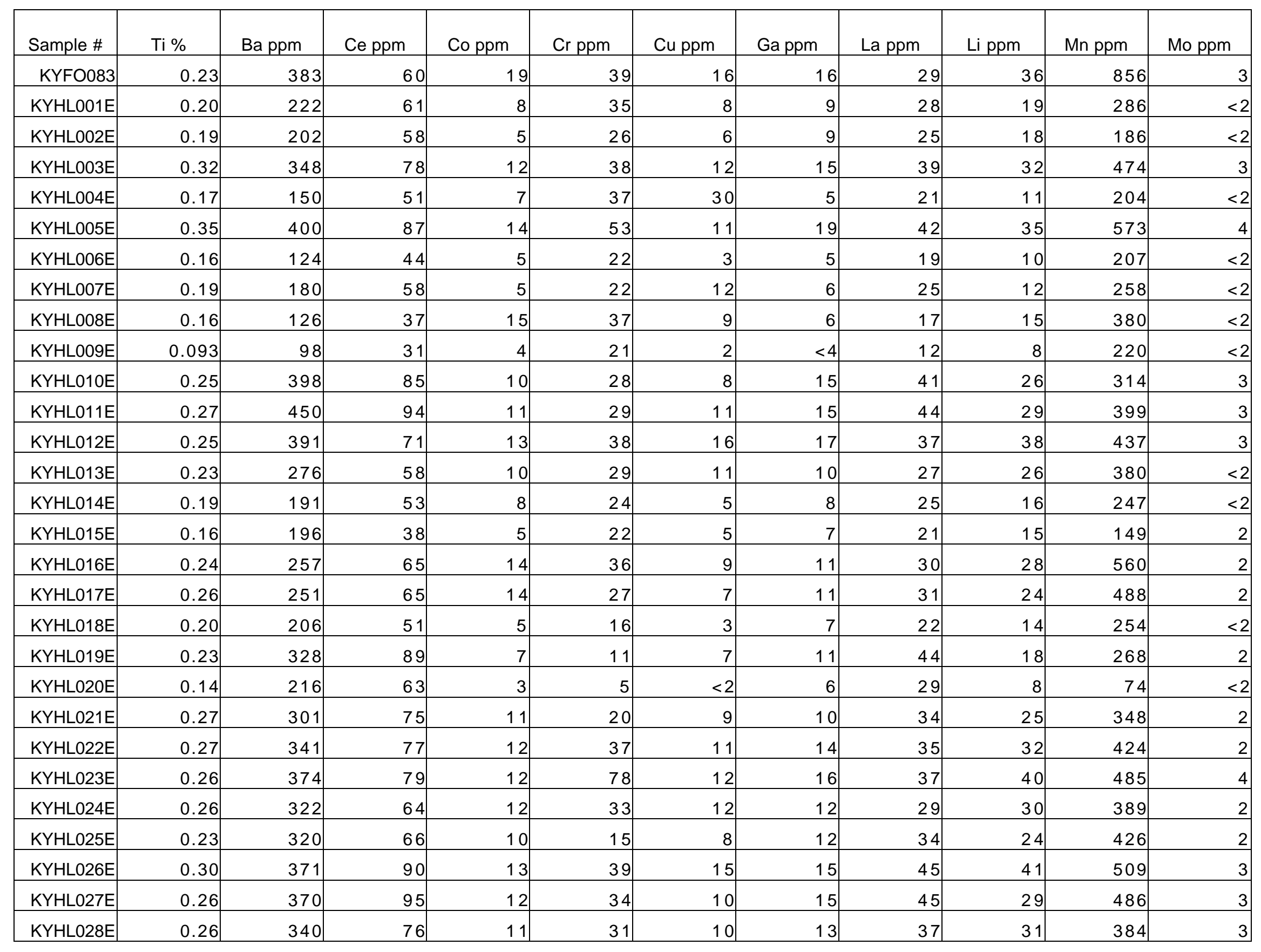




\begin{tabular}{|c|c|c|c|c|c|c|c|c|c|c|c|}
\hline Sample \# & $\mathrm{Ti} \%$ & Ba ppm & Ce ppm & Co ppm & Cr ppm & Cu ppm & Ga ppm & La ppm & Li ppm & Mn ppm & Mo ppm \\
\hline KYHL029E & 0.20 & 255 & 53 & 10 & 23 & 18 & 11 & 26 & 29 & 340 & 3 \\
\hline KYHL030E & 0.23 & 355 & 79 & 10 & 79 & 12 & 13 & 34 & 28 & 486 & 3 \\
\hline KYHL032E & 0.21 & 302 & 53 & 11 & 45 & 15 & 13 & 27 & 24 & 312 & 2 \\
\hline KYHL033E & 0.24 & 313 & 65 & 10 & 30 & 7 & 12 & 28 & 27 & 353 & 3 \\
\hline KYHL036E & 0.22 & 268 & 68 & 9 & 31 & 8 & 11 & 32 & 29 & 339 & 2 \\
\hline KYHL037E & 0.31 & 362 & 80 & 12 & 33 & 10 & 13 & 39 & 31 & 505 & 3 \\
\hline KYHL038E & 0.31 & 541 & 83 & 16 & 45 & 14 & 17 & 41 & 47 & 522 & 4 \\
\hline KYHL039E & 0.26 & 205 & 65 & 7 & 14 & 4 & 7 & 27 & 17 & 236 & $<2$ \\
\hline KYHL040E & 0.23 & 242 & 48 & 9 & 15 & 6 & 9 & 24 & 20 & 412 & $<2$ \\
\hline KYJA004 & 0.10 & 118 & 31 & 8 & 20 & $<2$ & 7 & 12 & 20 & 710 & $<2$ \\
\hline KYJA005 & 0.064 & 57 & 18 & 4 & 5 & $<2$ & $<4$ & 10 & 8 & 224 & $<2$ \\
\hline KYJA006 & 0.035 & 35 & 17 & $<2$ & 3 & $<2$ & $<4$ & 5 & 6 & 112 & $<2$ \\
\hline KYJA007 & 0.058 & 57 & 20 & 5 & 4 & $<2$ & $<4$ & 8 & 8 & 315 & $<2$ \\
\hline KYJA008 & 0.041 & 45 & 17 & 7 & 3 & $<2$ & $<4$ & 7 & 6 & 525 & $<2$ \\
\hline KYJA009 & 0.058 & 51 & 24 & 6 & 18 & 2 & $<4$ & 9 & 10 & 329 & $<2$ \\
\hline KYJA009F & 0.006 & 41 & 43 & 7 & 6 & 3 & $<4$ & 20 & 12 & 454 & $<2$ \\
\hline KYJA010 & 0.058 & 59 & 24 & 5 & 13 & 2 & $<4$ & 8 & 10 & 385 & $<2$ \\
\hline KYJA011 & 0.22 & 291 & 67 & 23 & 55 & 3 & 17 & 29 & 54 & 1270 & 4 \\
\hline KYJA012 & 0.25 & 263 & 68 & 22 & 53 & $<2$ & 14 & 27 & 33 & 1490 & 3 \\
\hline
\end{tabular}




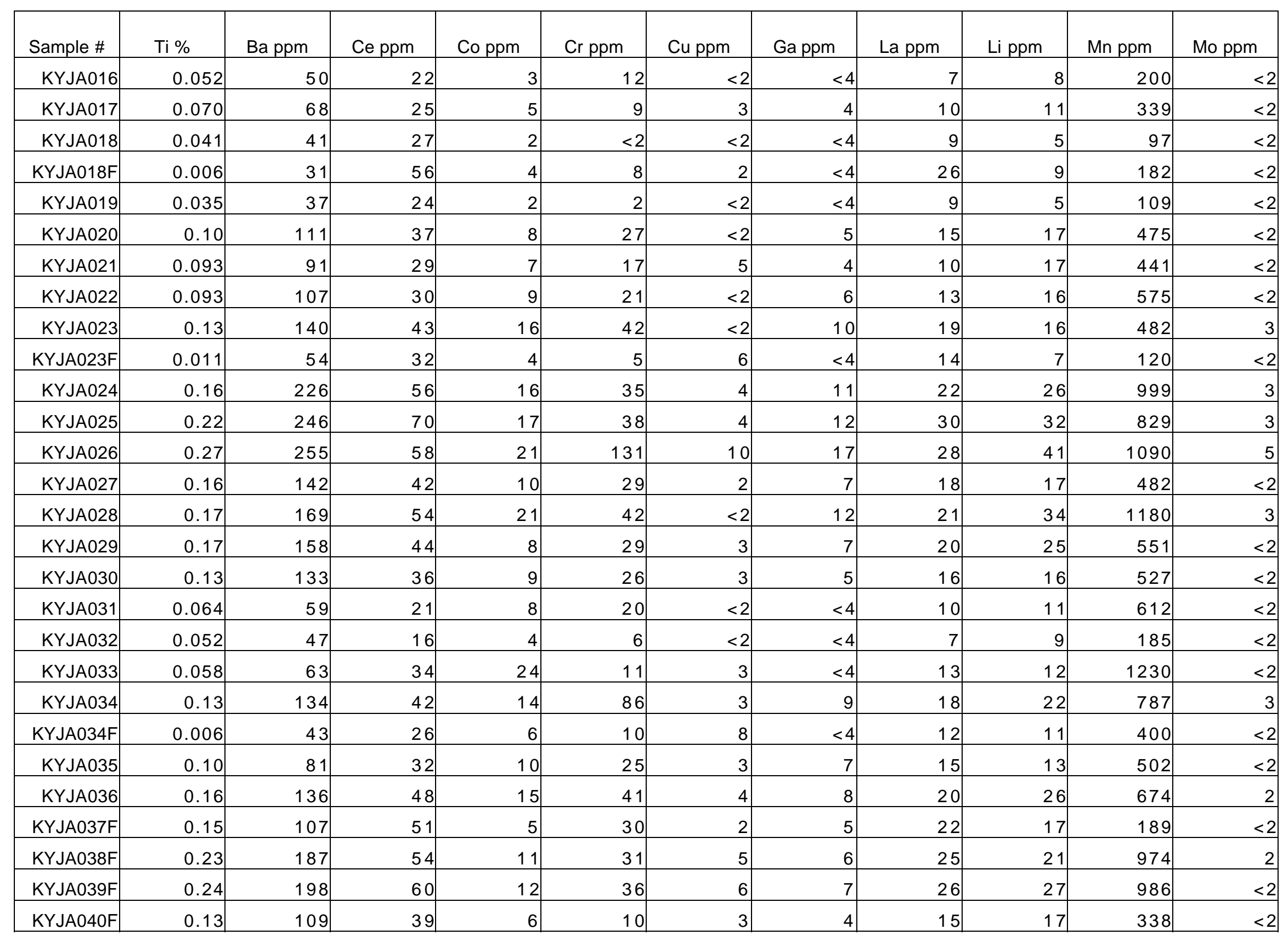




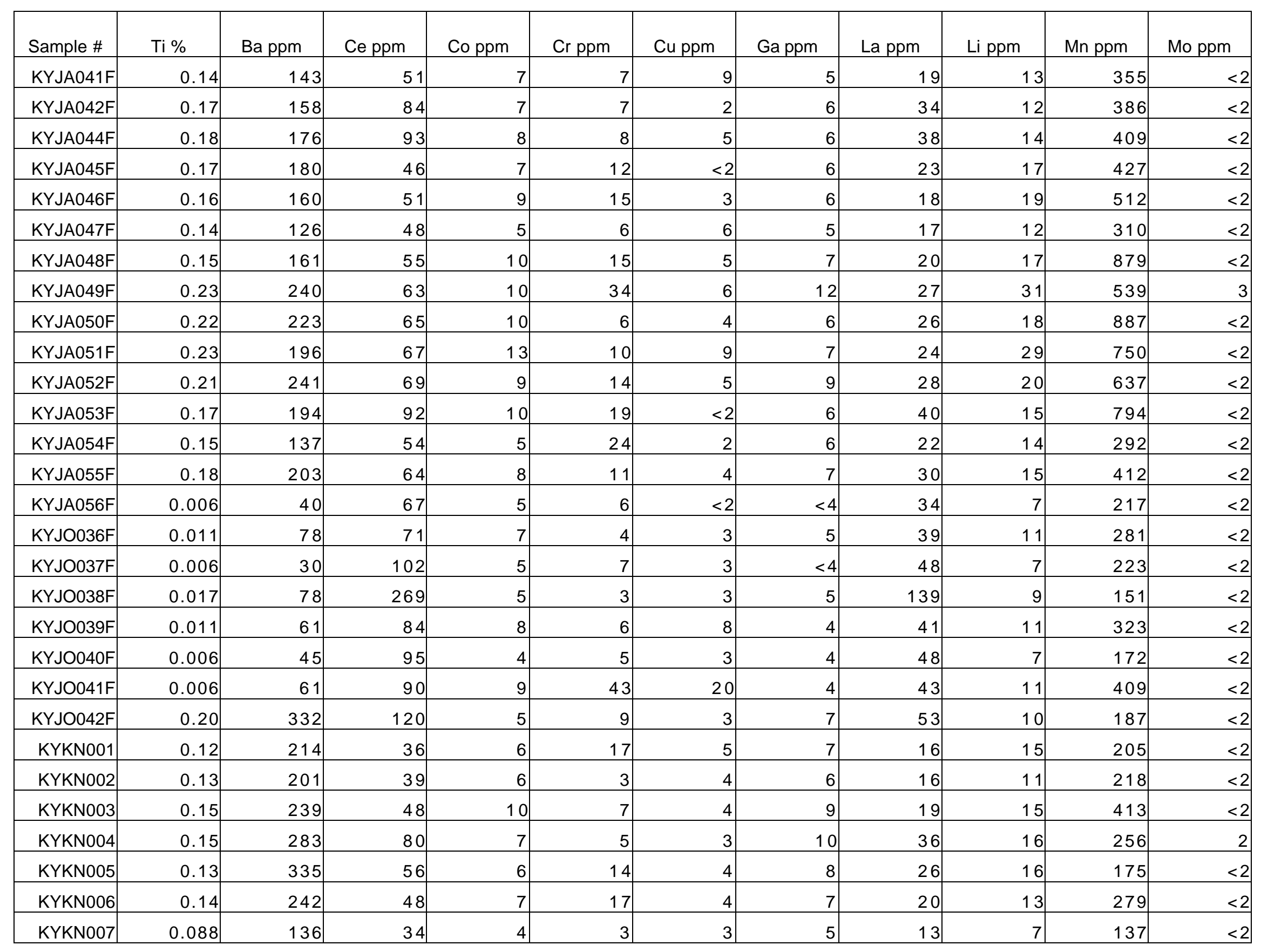




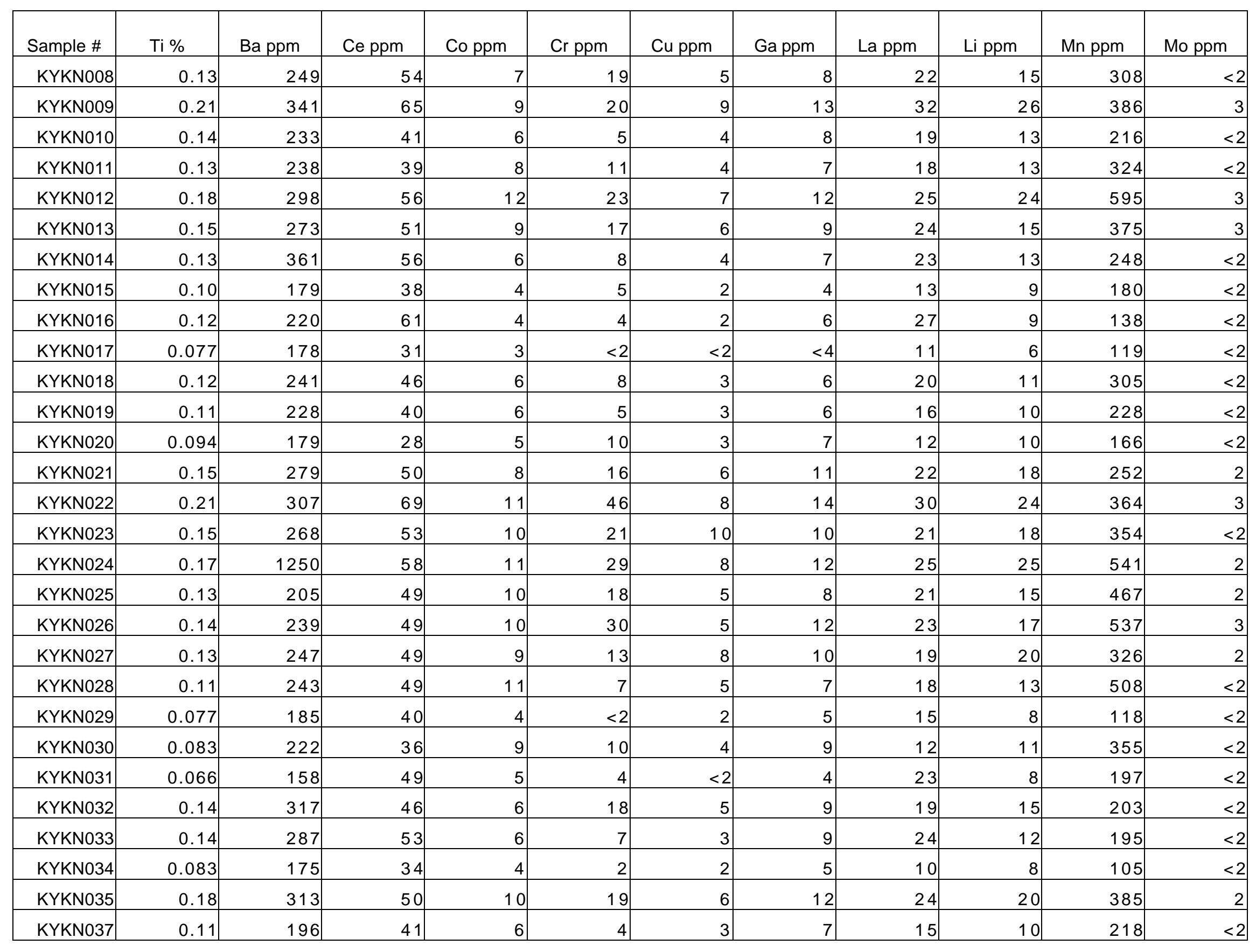




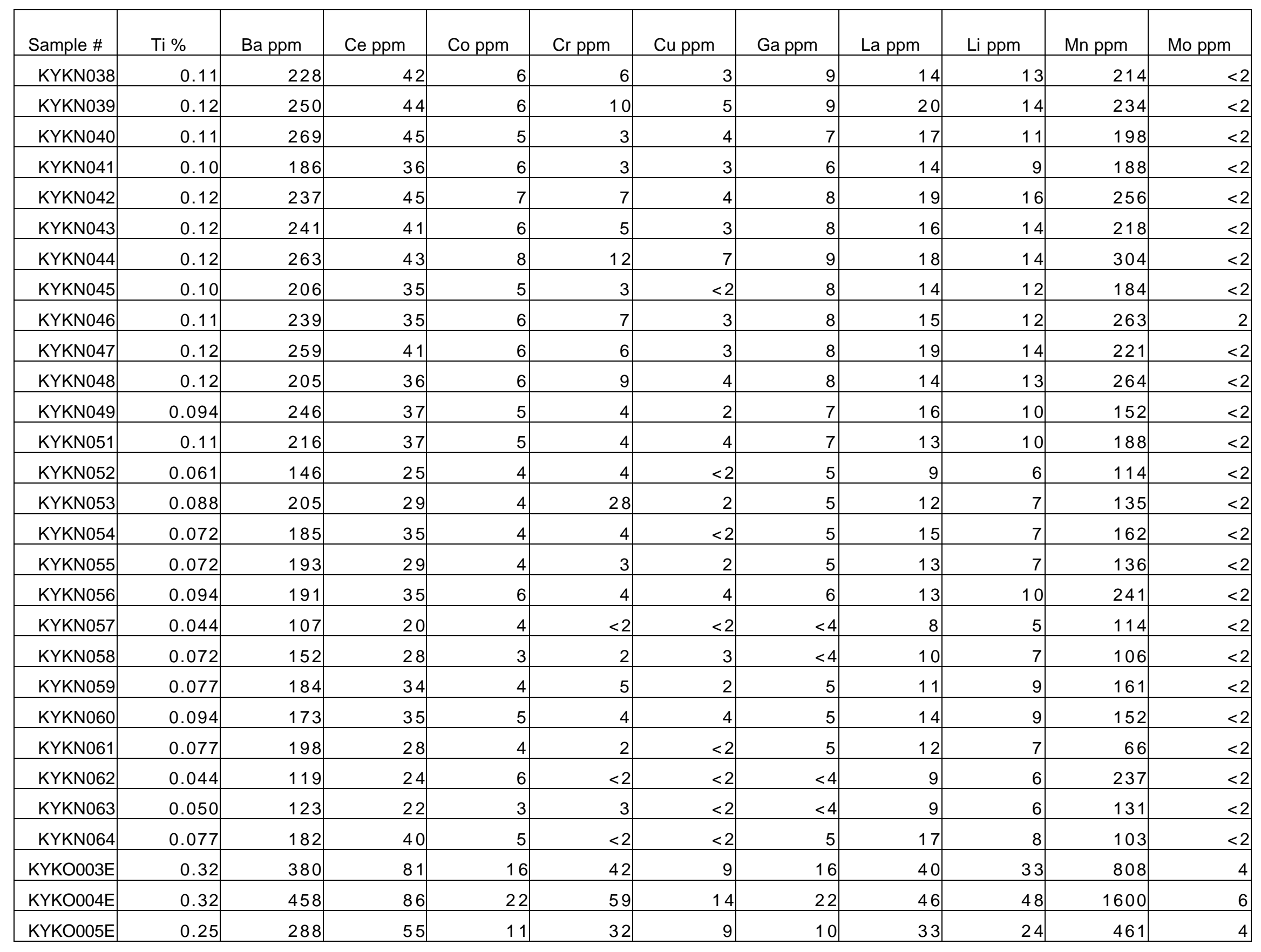




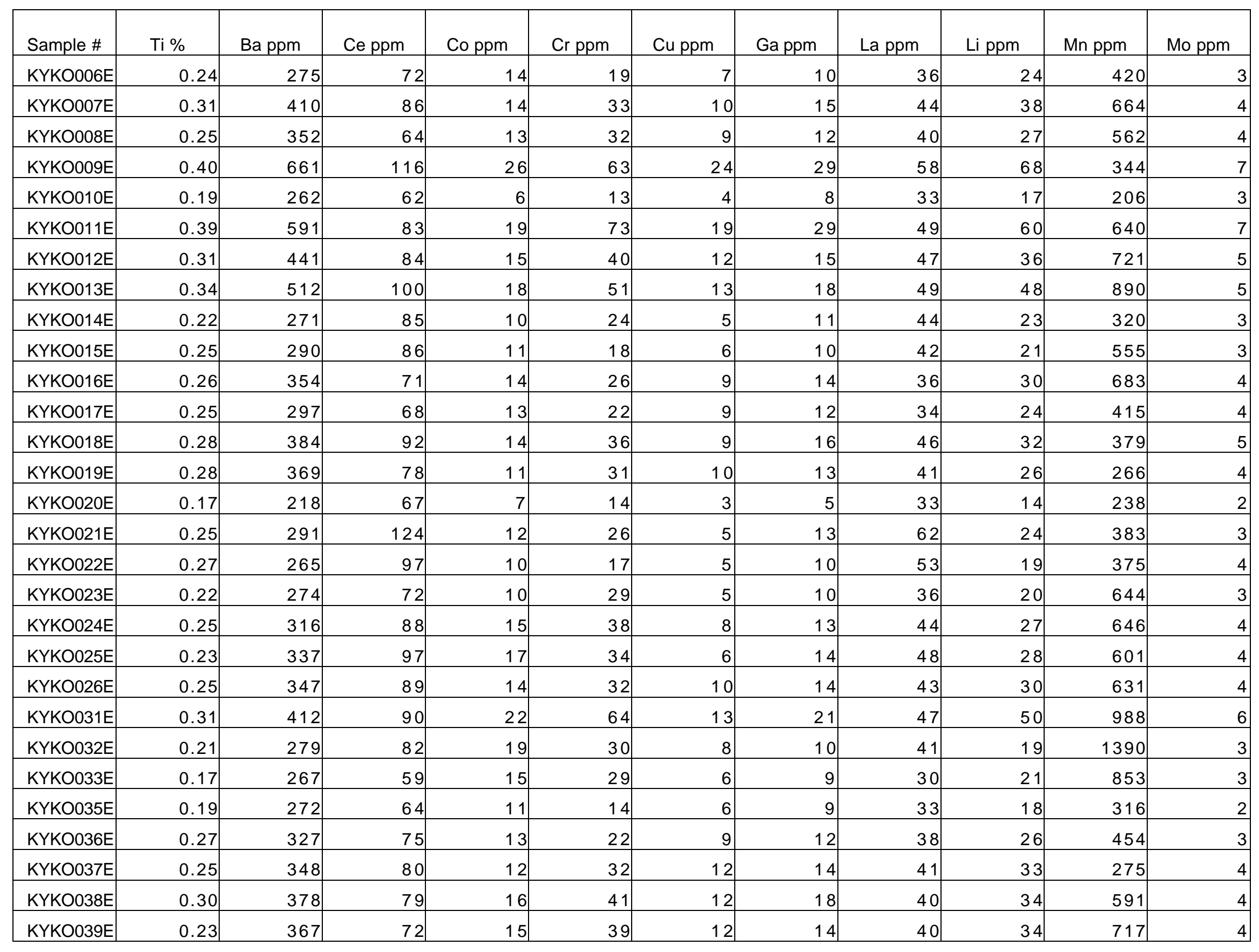




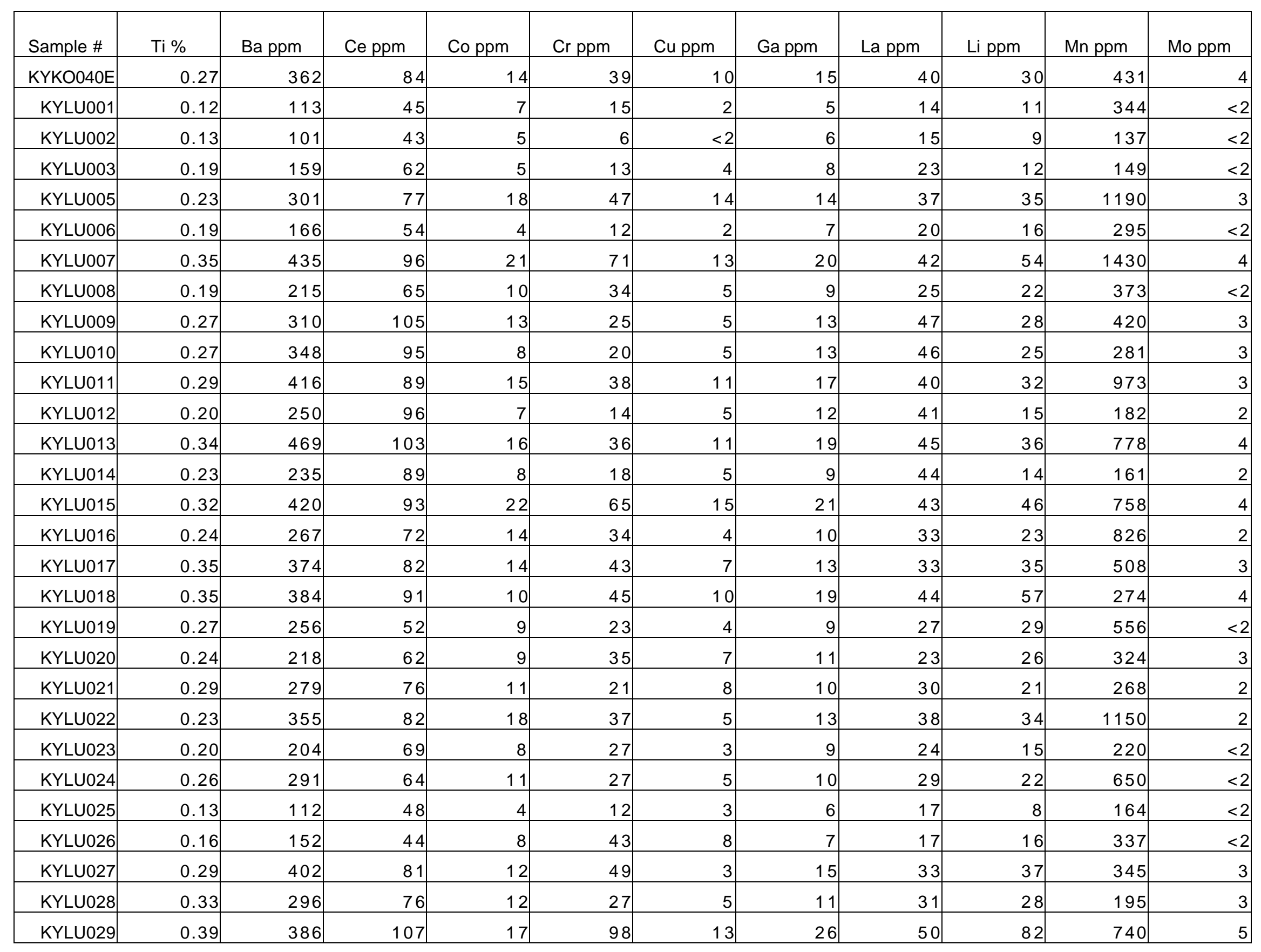




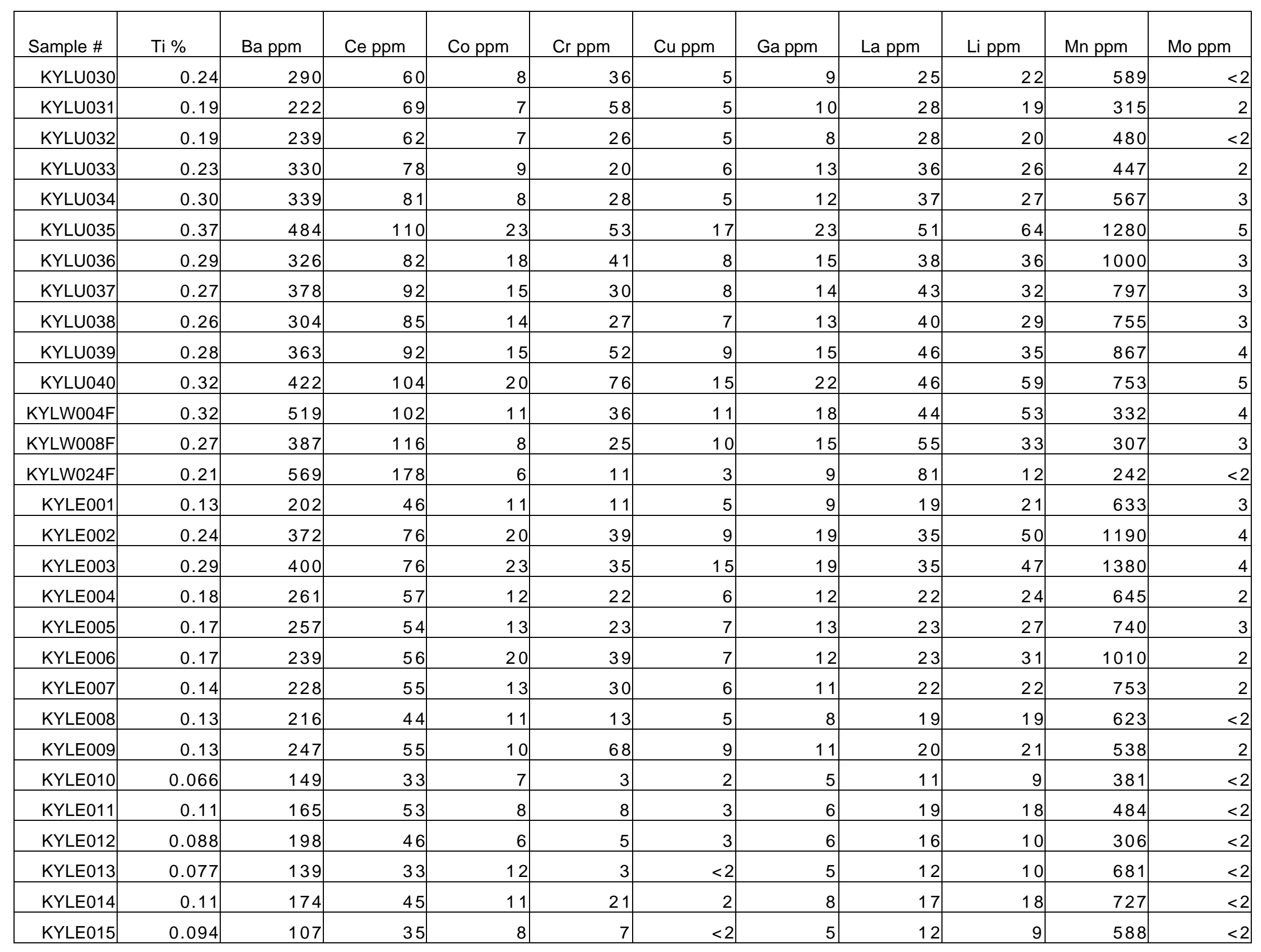




\begin{tabular}{|c|c|c|c|c|c|c|c|c|c|c|c|}
\hline KYLE017 & 0.23 & 299 & 68 & 19 & 45 & 5 & 15 & 29 & 35 & 1190 & 3 \\
\hline KYLE019 & 0.15 & 186 & 42 & 14 & 35 & 4 & 9 & 20 & 20 & 647 & 2 \\
\hline KYLE020 & 0.20 & 226 & 62 & 14 & 40 & 5 & 12 & 25 & 29 & 871 & 2 \\
\hline KYLE023 & 0.21 & 215 & 60 & 19 & 29 & 3 & 12 & 23 & 28 & 837 & 3 \\
\hline KYLE024 & 0.21 & 246 & 62 & 16 & 64 & 4 & 14 & 27 & 35 & 950 & 3 \\
\hline KYLE025 & 0.20 & 245 & 64 & 28 & 43 & 7 & 16 & 27 & 40 & 1570 & 3 \\
\hline KYLE026 & 0.20 & 261 & 63 & 18 & 65 & 7 & 13 & 25 & 39 & 1210 & 3 \\
\hline KYLE027 & 0.26 & 277 & 74 & 21 & 77 & 11 & 20 & 30 & 48 & 1120 & 4 \\
\hline KYLE031 & 0.19 & 225 & 59 & 10 & 23 & 4 & 11 & 26 & 25 & 465 & 2 \\
\hline KYLE032 & 0.10 & 110 & 26 & 7 & 6 & 3 & 7 & 14 & 24 & 477 & $<2$ \\
\hline KYLE033 & 0.036 & 43 & 10 & 2 & 2 & $<2$ & $<4$ & 6 & 9 & 74 & $<2$ \\
\hline KYLE034 & 0.042 & 52 & 15 & 2 & 3 & $<2$ & $<4$ & 6 & 7 & 83 & $<2$ \\
\hline KYLE035 & 0.024 & 235 & $<5$ & $<2$ & 2 & $<2$ & $<4$ & 4 & 3 & 28 & $<2$ \\
\hline KYLE036 & 0.018 & 72 & 10 & $<2$ & $<2$ & $<2$ & $<4$ & 4 & 3 & 169 & $<2$ \\
\hline KYLE037 & 0.024 & 24 & $<5$ & 2 & $<2$ & $<2$ & $<4$ & 3 & 4 & 90 & $<2$ \\
\hline KYLE038 & 0.024 & 27 & $<5$ & 2 & 5 & $<2$ & $<4$ & 6 & 4 & 113 & $<2$ \\
\hline KYLE039 & 0.036 & 37 & 9 & 3 & 5 & $<2$ & $<4$ & 5 & 6 & 150 & $<2$ \\
\hline KYLE040 & 0.21 & 231 & 57 & 20 & 64 & 5 & 14 & 26 & 40 & 1280 & 3 \\
\hline
\end{tabular}




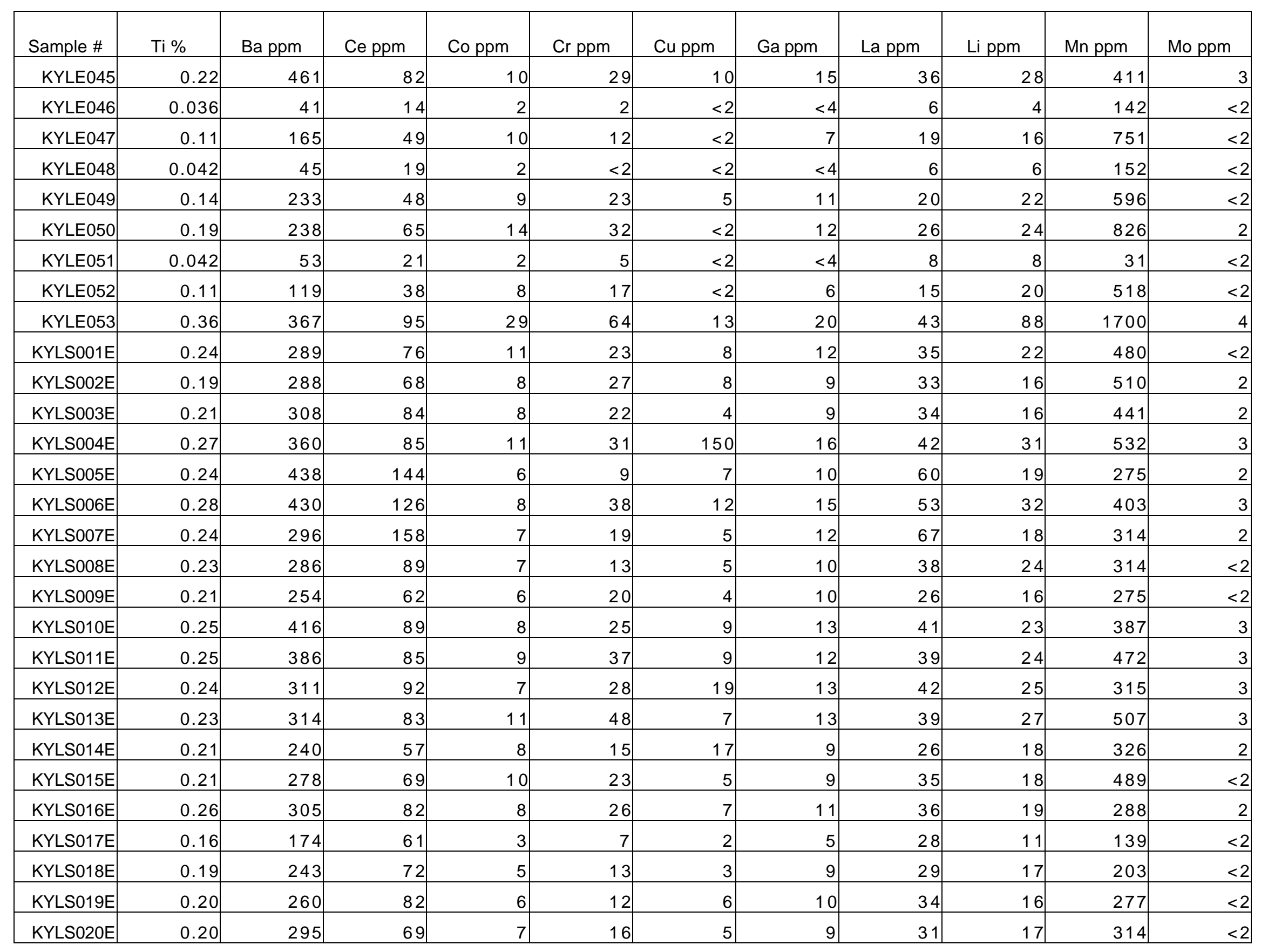




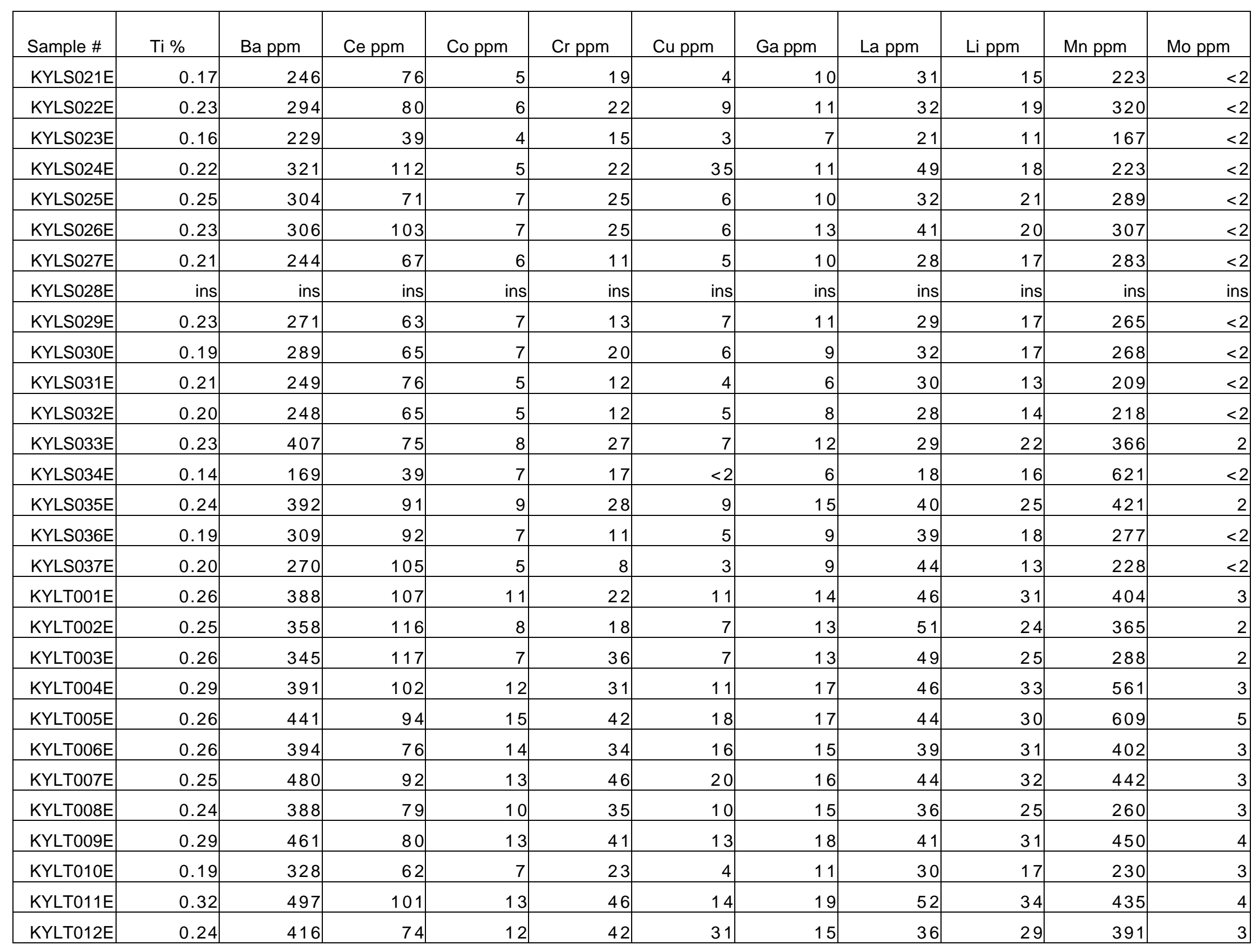




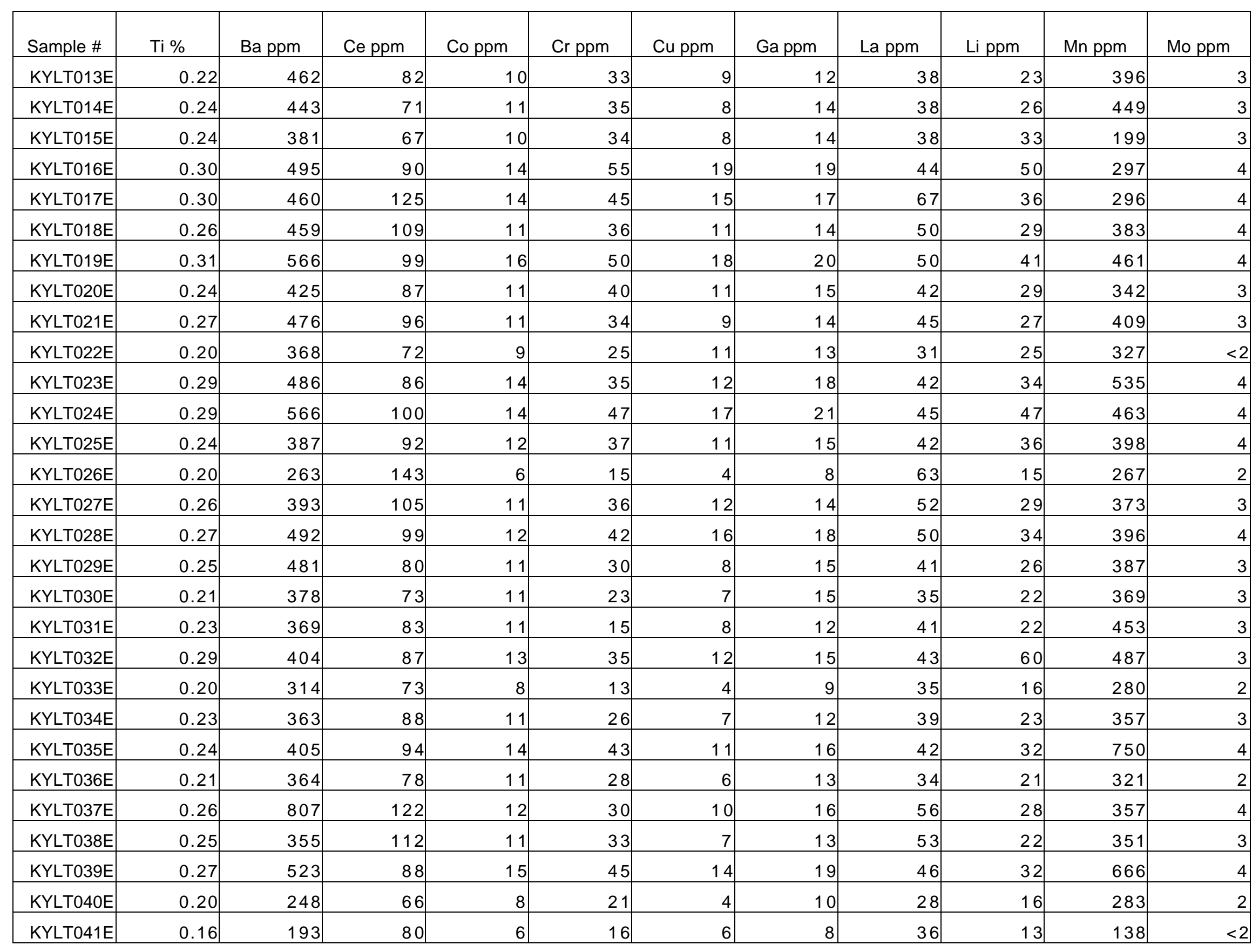




\begin{tabular}{|c|c|c|c|c|c|c|c|c|c|c|c|}
\hline Sample \# & $\mathrm{Ti} \%$ & Ba ppm & Ce ppm & Co ppm & Cr ppm & Cu ppm & Ga ppm & La ppm & Li ppm & Mn ppm & Mo ppm \\
\hline KYMG001F & 0.19 & 260 & 63 & 7 & 19 & 4 & 8 & 27 & 15 & 264 & $<2$ \\
\hline KYMG002F & 0.24 & 279 & 82 & 8 & 21 & 6 & 12 & 35 & 19 & 299 & $<2$ \\
\hline KYMG004F & 0.16 & 280 & 114 & 7 & 14 & 4 & 8 & 49 & 15 & 315 & 2 \\
\hline KYMG005F & 0.22 & 286 & 89 & 10 & 27 & 8 & 13 & 36 & 23 & 379 & 2 \\
\hline KYMG008F & 0.22 & 306 & 75 & 8 & 40 & 9 & 9 & 29 & 17 & 229 & 10 \\
\hline KYMG009F & 0.23 & 341 & 88 & 9 & 27 & 9 & 12 & 38 & 22 & 376 & $<2$ \\
\hline KYMG010F & 0.21 & 351 & 91 & 7 & 13 & 5 & 10 & 41 & 18 & 268 & $<2$ \\
\hline KYMG011F & 0.20 & 304 & 85 & 6 & 15 & 5 & 8 & 36 & 15 & 223 & $<2$ \\
\hline KYMG012F & 0.22 & 319 & 90 & 11 & 27 & 6 & 11 & 37 & 22 & 546 & 2 \\
\hline KYMG017F & 0.13 & 142 & 45 & 3 & 3 & $<2$ & 5 & 16 & 6 & 121 & $<2$ \\
\hline KYMG018F & 0.27 & 343 & 109 & 13 & 38 & 9 & 17 & 45 & 28 & 514 & 3 \\
\hline KYMG019F & 0.26 & 328 & 135 & 9 & 21 & 6 & 14 & 57 & 25 & 359 & $<2$ \\
\hline KYMG020F & 0.11 & 153 & 47 & 5 & 16 & 5 & 7 & 16 & 9 & 209 & 2 \\
\hline KYMG021F & 0.30 & 398 & 110 & 13 & 44 & 16 & 19 & 48 & 37 & 455 & 3 \\
\hline KYMG022F & 0.29 & 311 & 398 & 8 & 54 & 7 & 13 & 179 & 22 & 345 & $<2$ \\
\hline KYMG023F & 0.24 & 326 & 94 & 9 & 28 & 6 & 11 & 45 & 22 & 413 & 3 \\
\hline KYMG024F & 0.29 & 400 & 101 & 14 & 46 & 10 & 16 & 49 & 41 & 680 & 4 \\
\hline KYMG025F & 0.20 & 320 & 89 & 10 & 20 & 5 & 12 & 40 & 21 & 350 & 3 \\
\hline KYMG026F & 0.21 & 327 & 81 & 7 & 37 & 6 & 10 & 35 & 16 & 206 & 2 \\
\hline
\end{tabular}




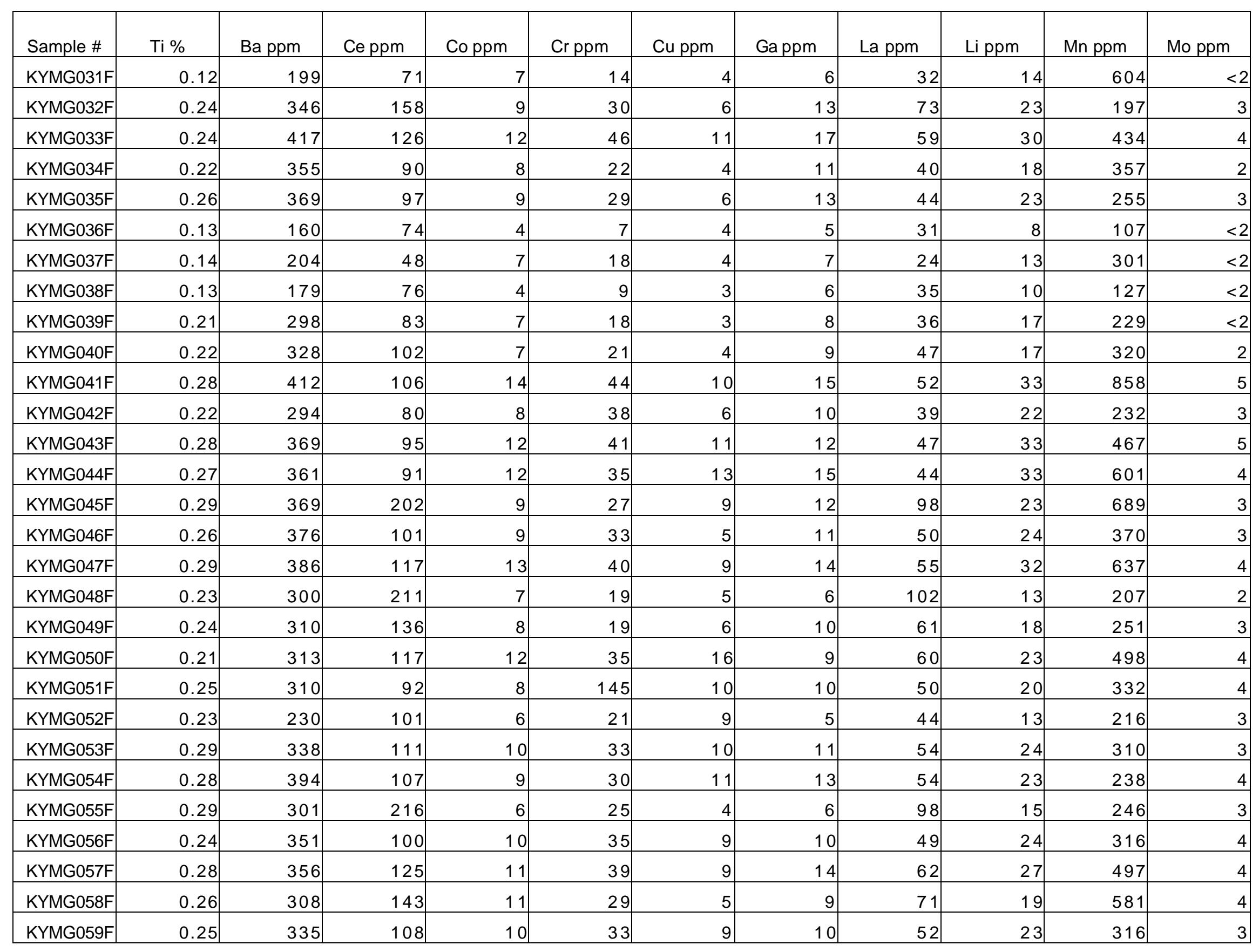




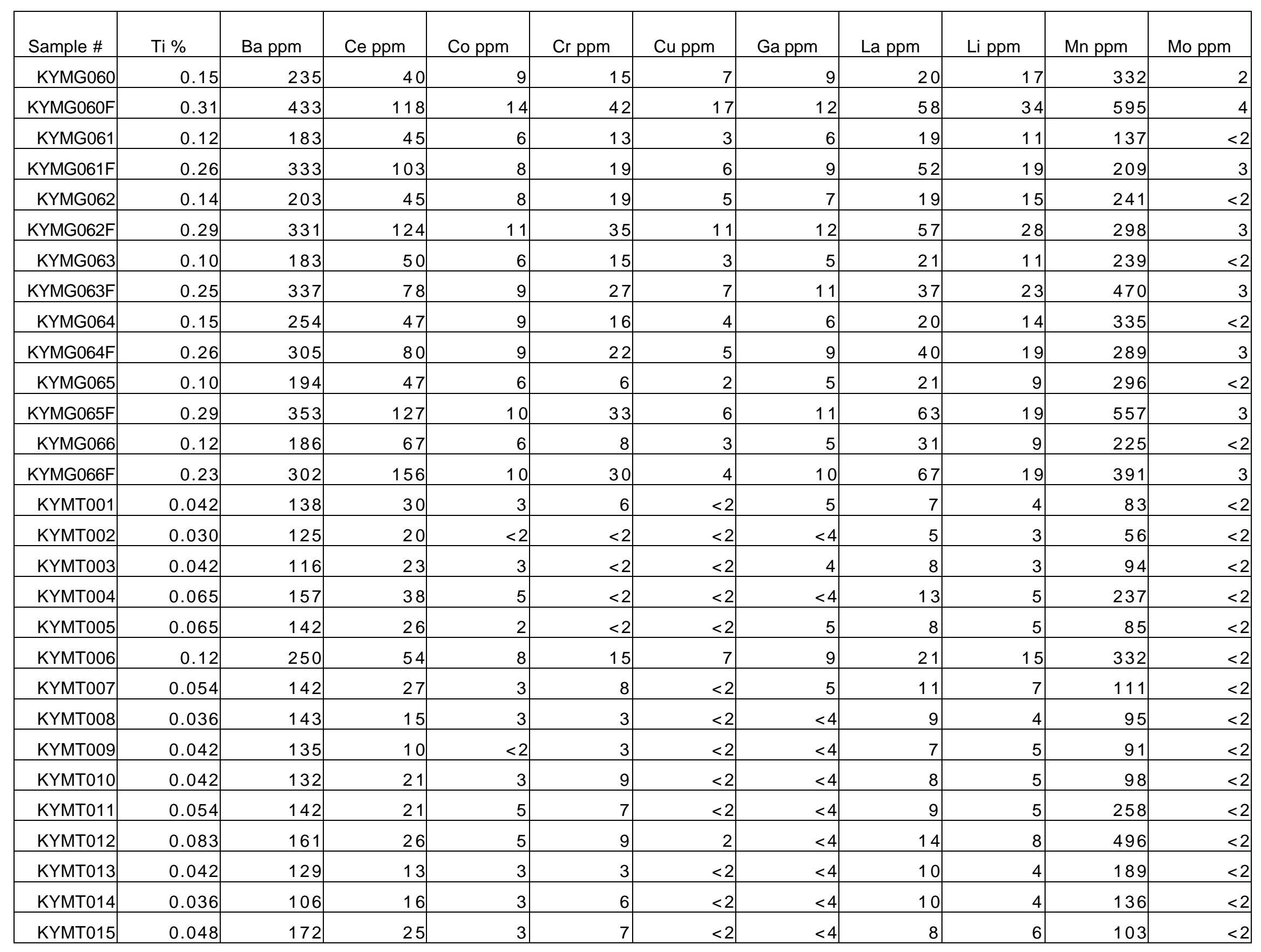




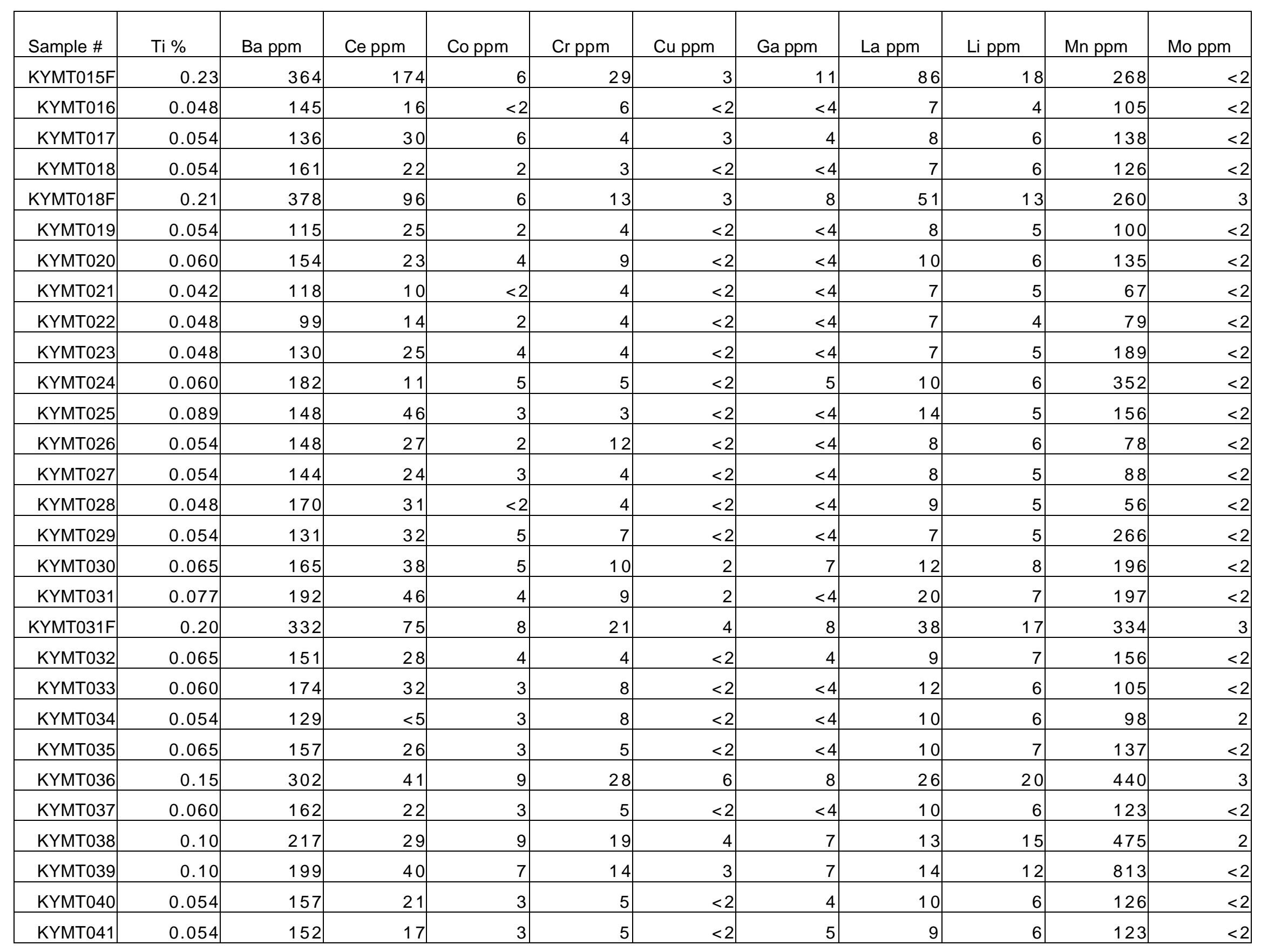




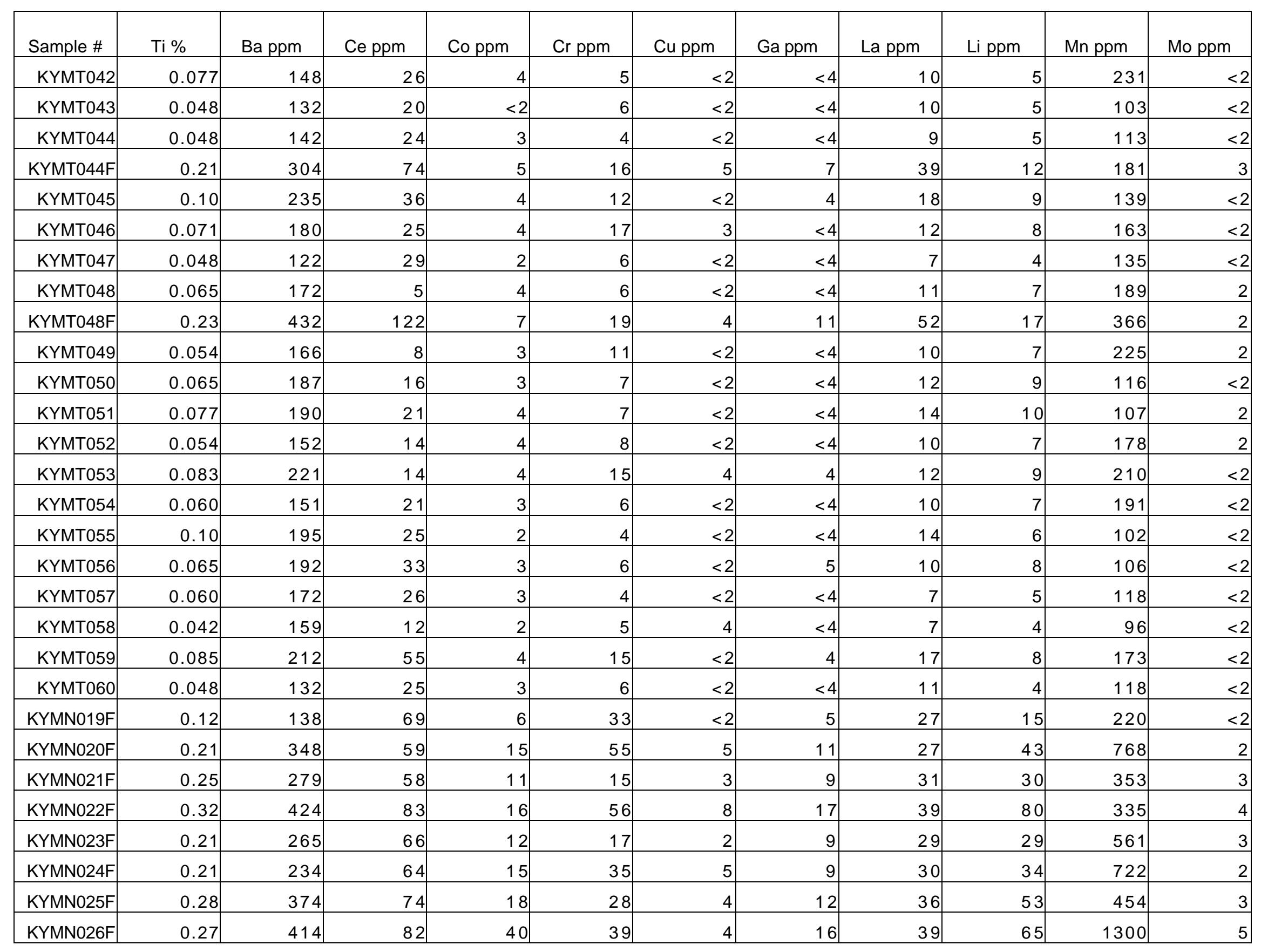




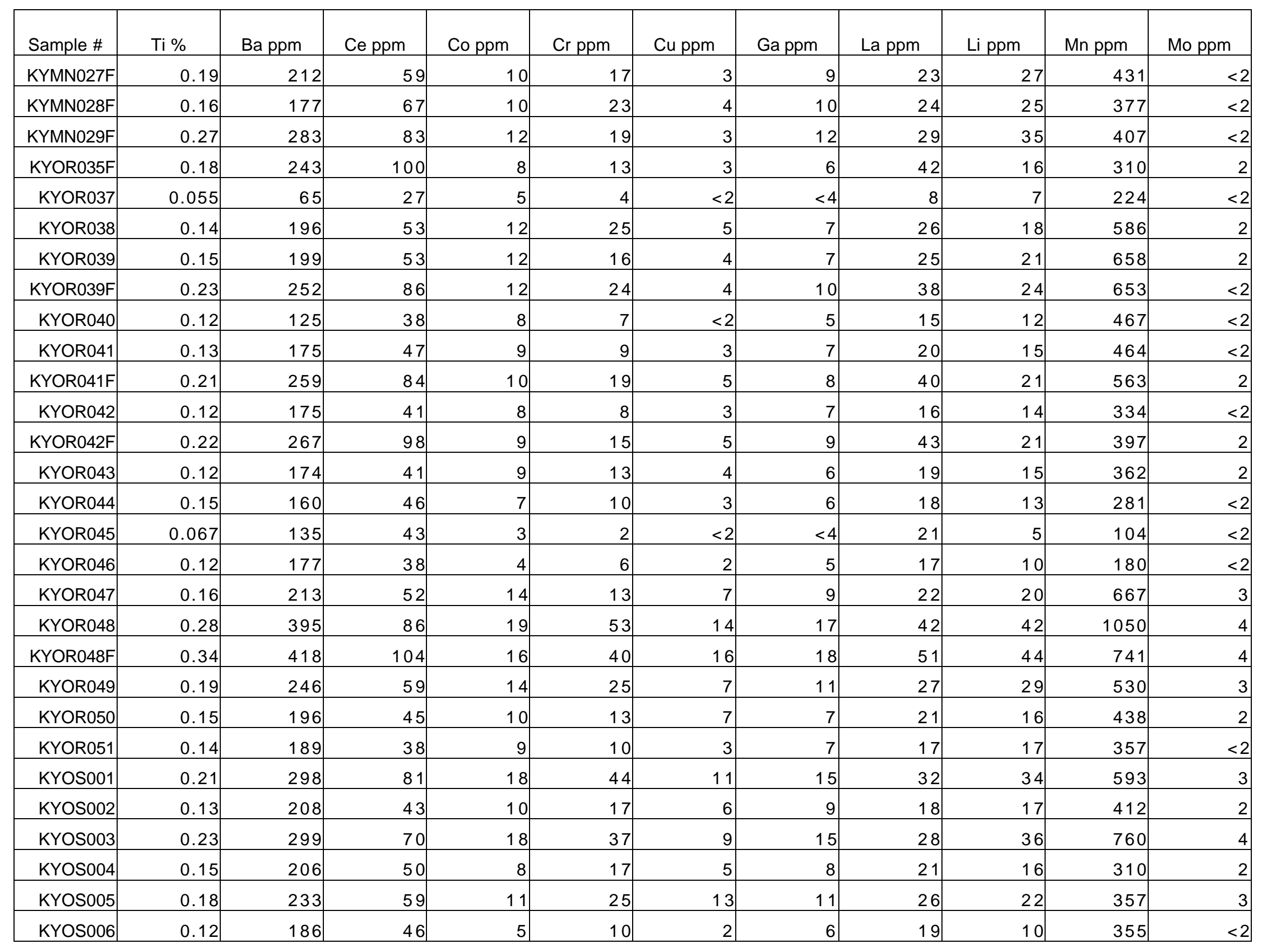




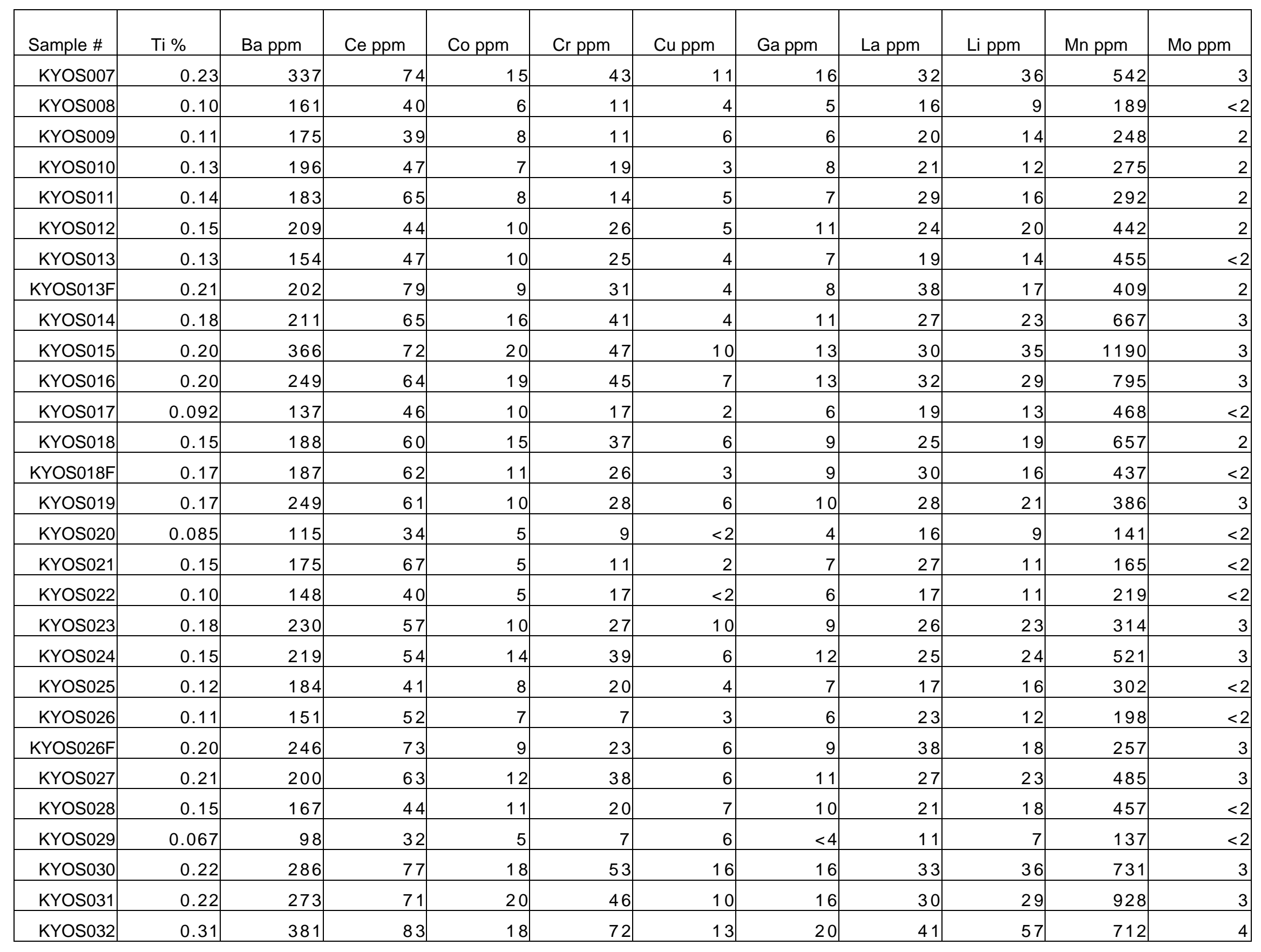




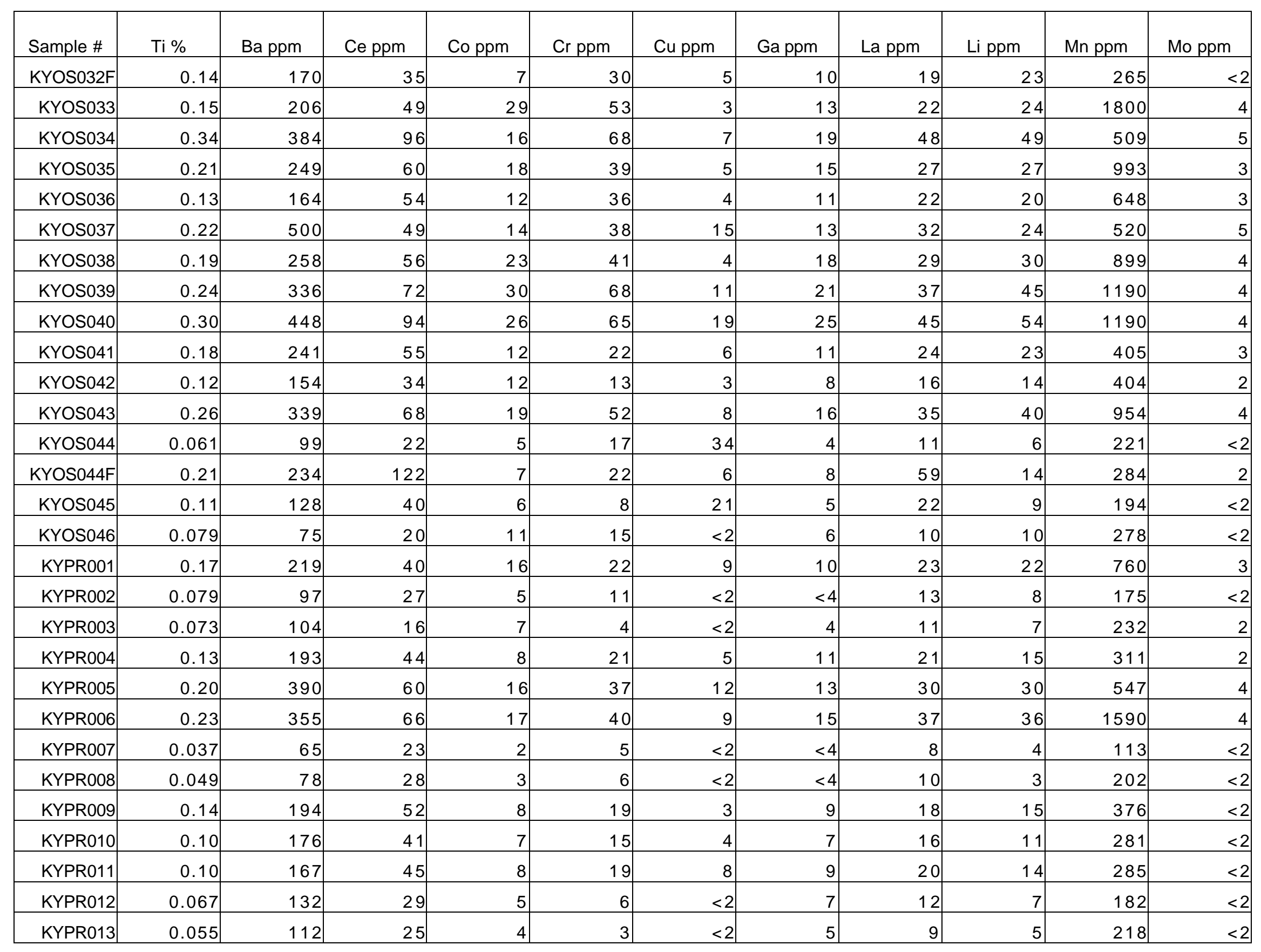




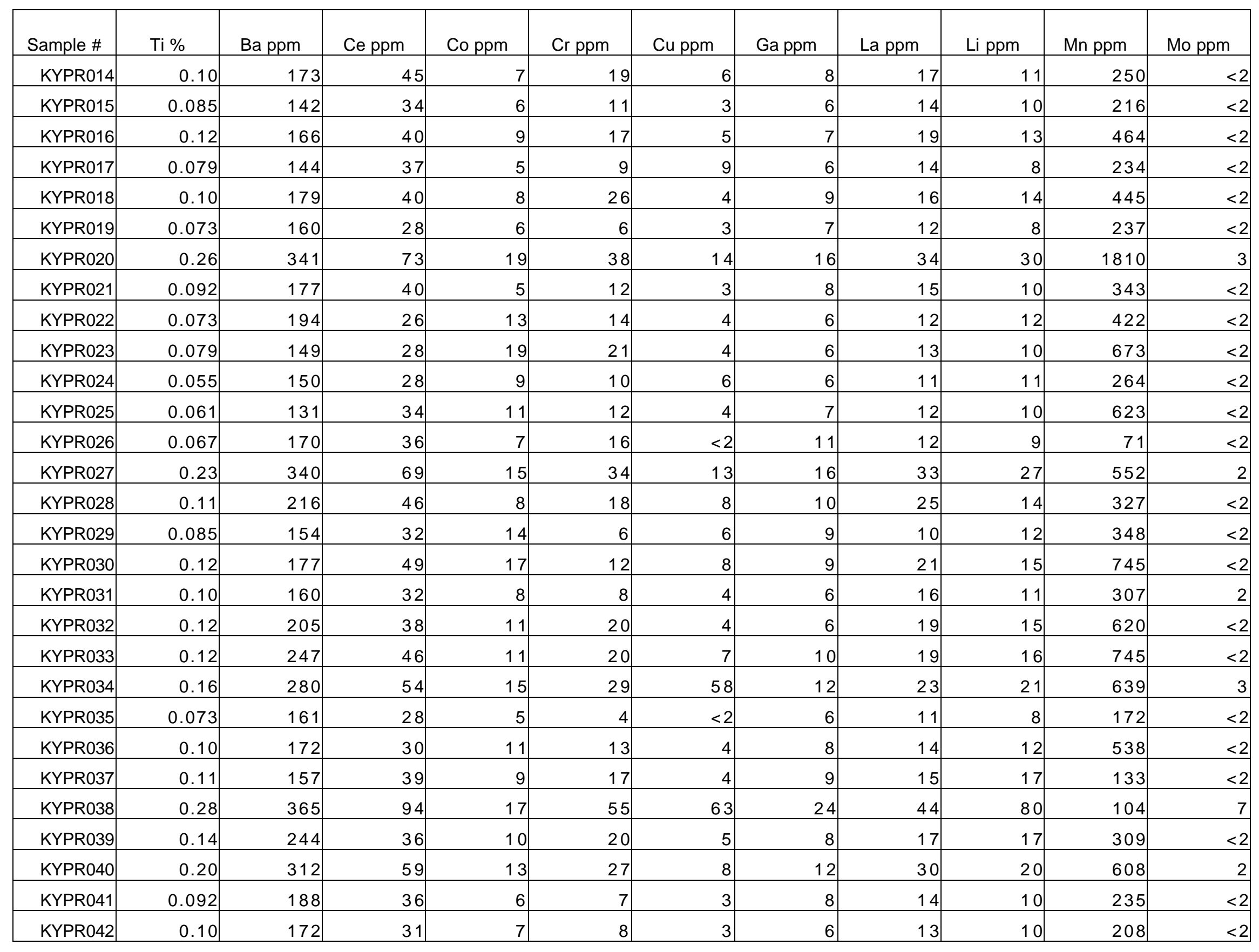




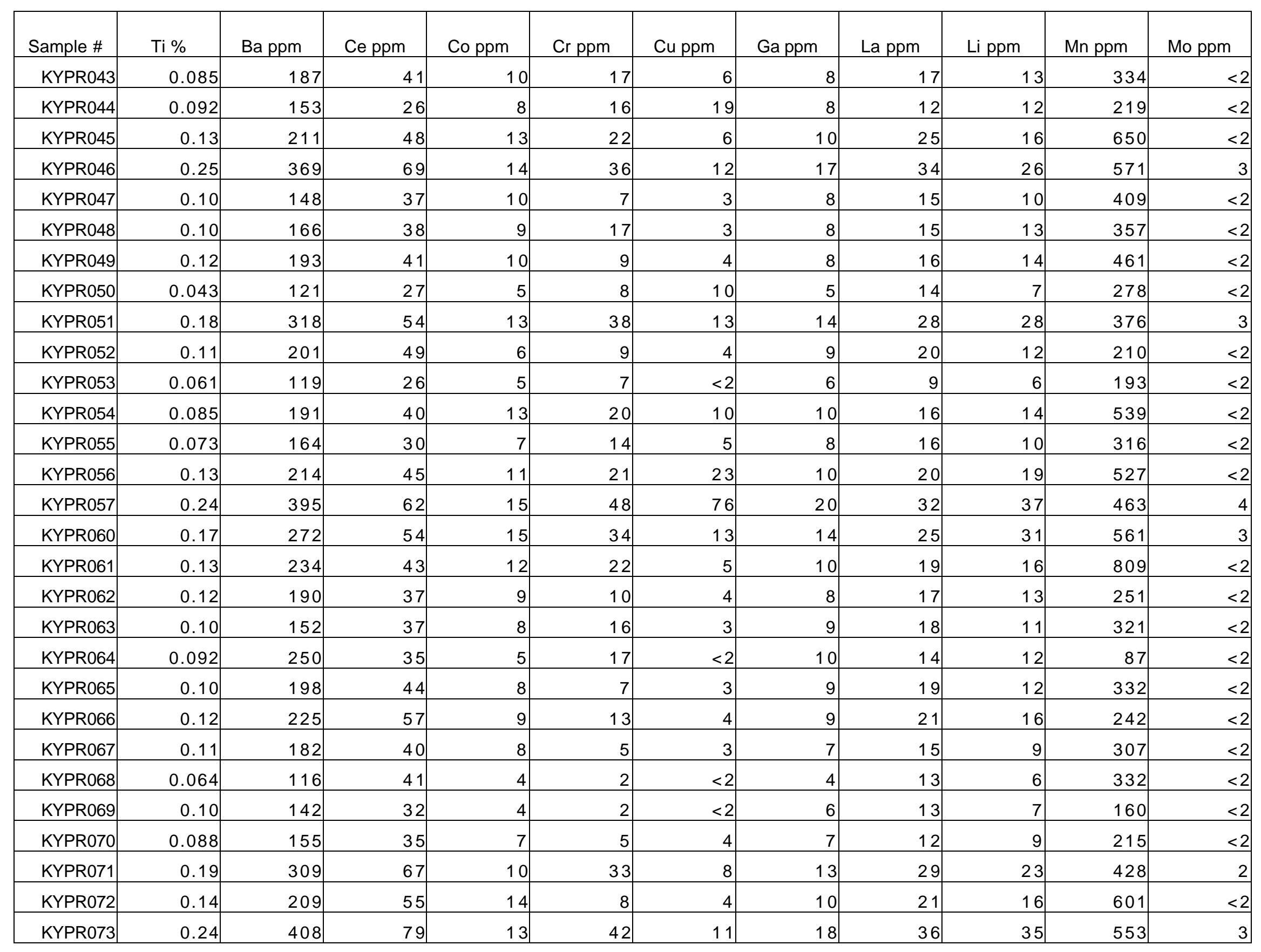




\begin{tabular}{|c|c|c|c|c|c|c|c|c|c|c|c|}
\hline Sample \# & $\mathrm{Ti} \%$ & Ba ppm & Ce ppm & Co ppm & Cr ppm & Cu ppm & Ga ppm & La ppm & Li ppm & Mn ppm & Mo ppm \\
\hline KYPR074 & 0.27 & 465 & 89 & 15 & 43 & 13 & 20 & 42 & 42 & 1060 & 4 \\
\hline KYPR075 & 0.088 & 163 & 37 & 4 & 11 & $<2$ & 6 & 13 & 9 & 209 & $<2$ \\
\hline KYPR077 & 0.14 & 240 & 49 & 6 & 13 & 3 & 9 & 18 & 13 & 295 & $<2$ \\
\hline KYPR078 & 0.10 & 189 & 46 & 5 & 6 & 4 & 8 & 15 & 9 & 251 & $<2$ \\
\hline KYPR081 & 0.070 & 122 & 31 & 4 & 4 & $<2$ & 6 & 10 & 6 & 280 & $<2$ \\
\hline KYPR082 & 0.20 & 303 & 55 & 9 & 26 & 6 & 11 & 29 & 26 & 316 & $<2$ \\
\hline KYPR083 & 0.076 & 117 & 32 & 5 & 7 & $<2$ & 5 & 10 & 9 & 309 & $<2$ \\
\hline KYPR084 & 0.088 & 145 & 40 & 4 & 5 & $<2$ & 6 & 14 & 8 & 345 & $<2$ \\
\hline KYPR085 & 0.070 & 143 & 34 & 7 & 12 & 2 & 6 & 11 & 9 & 376 & $<2$ \\
\hline KYPI002 & 0.11 & 233 & 41 & 7 & 7 & 4 & 8 & 16 & 12 & 221 & $<2$ \\
\hline KYPI003 & 0.088 & 190 & 32 & 5 & $<2$ & $<2$ & 6 & 11 & 8 & 166 & $<2$ \\
\hline KYPI004 & 0.12 & 222 & 39 & 7 & $<2$ & $<2$ & 8 & 16 & 11 & 234 & $<2$ \\
\hline KYPI004F & 0.26 & 384 & 99 & 9 & 23 & 5 & 13 & 43 & 25 & 468 & 2 \\
\hline KYPI005 & 0.14 & 249 & 44 & 8 & 3 & 4 & 8 & 19 & 12 & 274 & $<2$ \\
\hline KYPI006 & 0.18 & 269 & 57 & 7 & 3 & 4 & 11 & 22 & 13 & 220 & $<2$ \\
\hline KYPI007 & 0.064 & 189 & 34 & 4 & 3 & $<2$ & 6 & 12 & 8 & 150 & $<2$ \\
\hline KYPI008 & 0.070 & 223 & 29 & 5 & 3 & $<2$ & 6 & 12 & 8 & 154 & $<2$ \\
\hline KYPI009 & 0.070 & 183 & 25 & 4 & 2 & $<2$ & 6 & 11 & 7 & 161 & $<2$ \\
\hline KYPI010 & 0.12 & 234 & 39 & 7 & 13 & 3 & 10 & 17 & 12 & 250 & $<2$ \\
\hline
\end{tabular}




\begin{tabular}{|c|c|c|c|c|c|c|c|c|c|c|c|}
\hline Sample \# & $\mathrm{Ti} \%$ & Ba ppm & Ce ppm & Co ppm & Cr ppm & Cu ppm & Ga ppm & La ppm & Li ppm & Mn ppm & Mo ppm \\
\hline KYPI016 & 0.10 & 213 & 39 & 5 & 3 & 7 & 8 & 17 & 11 & 191 & $<2$ \\
\hline KYPI018 & 0.12 & 241 & 48 & 10 & 10 & 7 & 10 & 20 & 19 & 336 & $<2$ \\
\hline KYPI018F & 0.27 & 372 & 74 & 14 & 41 & 32 & 12 & 33 & 32 & 522 & 3 \\
\hline KYPI020 & 0.14 & 233 & 44 & 9 & 5 & 6 & 8 & 17 & 13 & 289 & 2 \\
\hline KYPI021 & 0.12 & 235 & 53 & 6 & 6 & 4 & 11 & 20 & 16 & 150 & $<2$ \\
\hline KYPI022 & 0.082 & 209 & 33 & 6 & 5 & 2 & 7 & 15 & 8 & 169 & $<2$ \\
\hline KYPI023 & 0.11 & 236 & 34 & 6 & 10 & $<2$ & 8 & 17 & 12 & 228 & $<2$ \\
\hline KYPI024 & 0.11 & 224 & 42 & 7 & 3 & 3 & 8 & 14 & 13 & 353 & $<2$ \\
\hline KYPI067 & 0.088 & 180 & 29 & 5 & 3 & 5 & 5 & 11 & 9 & 148 & $<2$ \\
\hline KYPI068 & 0.094 & 198 & 42 & 6 & 5 & 4 & 7 & 17 & 12 & 202 & $<2$ \\
\hline KYPI069 & 0.18 & 292 & 50 & 12 & 15 & 9 & 12 & 23 & 23 & 538 & 3 \\
\hline KYPI070 & 0.12 & 214 & 38 & 7 & 6 & 7 & 7 & 18 & 15 & 238 & $<2$ \\
\hline KYPI071 & 0.12 & 247 & 41 & 7 & 6 & 4 & 6 & 19 & 14 & 270 & 2 \\
\hline KYPI072 & 0.12 & 238 & 38 & 6 & 5 & 3 & 8 & 16 & 12 & 233 & 2 \\
\hline KYPI073 & 0.11 & 221 & 29 & 6 & 4 & 3 & 6 & 14 & 12 & 243 & $<2$ \\
\hline KYPI074 & 0.10 & 229 & 33 & 5 & 6 & 3 & 7 & 14 & 12 & 194 & $<2$ \\
\hline KYPI075 & 0.13 & 233 & 36 & 9 & 5 & 4 & 8 & 15 & 15 & 318 & 2 \\
\hline KYPI076 & 0.13 & 235 & 36 & 6 & 4 & 5 & 9 & 16 & 15 & 263 & $<2$ \\
\hline
\end{tabular}




\begin{tabular}{|c|c|c|c|c|c|c|c|c|c|c|c|}
\hline KYPI082 & 0.11 & 183 & 27 & 5 & 8 & $<2$ & 9 & 15 & 15 & 214 & 2 \\
\hline KYPI084 & 0.30 & 1530 & 63 & 11 & 51 & 24 & 21 & 32 & 69 & 310 & 5 \\
\hline KYPI085 & 0.14 & 232 & 42 & 7 & 16 & 4 & 10 & 19 & 19 & 269 & 2 \\
\hline KYPI088 & 0.14 & 221 & 43 & 9 & 24 & 4 & 11 & 21 & 21 & 436 & 4 \\
\hline KYPI089 & 0.16 & 229 & 48 & 11 & 28 & 5 & 11 & 21 & 23 & 479 & 4 \\
\hline KYPI090 & 0.14 & 319 & 42 & 7 & 23 & 3 & 9 & 21 & 21 & 351 & 3 \\
\hline KYPI091 & 0.14 & 188 & 43 & 6 & 22 & $<2$ & 9 & 24 & 18 & 326 & 3 \\
\hline KYPI092 & 0.16 & 280 & 27 & 11 & 10 & 3 & 10 & 21 & 21 & 486 & 3 \\
\hline KYPI096 & 0.16 & 393 & 52 & 9 & 28 & 8 & 12 & 27 & 26 & 274 & 4 \\
\hline KYPI097 & 0.12 & 242 & 30 & 6 & 25 & 5 & 10 & 17 & 27 & 220 & 3 \\
\hline KYPI098 & 0.13 & 253 & 33 & 6 & 12 & 4 & 10 & 18 & 19 & 167 & 3 \\
\hline KYPI099 & 0.11 & 257 & 27 & 6 & 14 & $<2$ & 8 & 16 & 13 & 219 & 3 \\
\hline KYPI100 & 0.10 & 209 & 24 & 5 & 9 & $<2$ & 7 & 15 & 13 & 185 & 2 \\
\hline KYPI101 & 0.15 & 265 & 43 & 8 & 13 & 4 & 10 & 26 & 17 & 299 & 3 \\
\hline KYPI102 & 0.12 & 234 & 25 & 5 & 9 & $<2$ & 8 & 16 & 14 & 226 & 3 \\
\hline KYPI103 & 0.14 & 329 & 45 & 10 & 27 & 6 & 13 & 25 & 22 & 404 & 3 \\
\hline KYPI104 & 0.10 & 219 & 25 & 5 & 6 & $<2$ & 8 & 17 & 12 & 155 & $<2$ \\
\hline KYPI105 & 0.11 & 190 & 31 & 5 & 14 & $<2$ & 7 & 18 & 13 & 248 & $<2$ \\
\hline
\end{tabular}




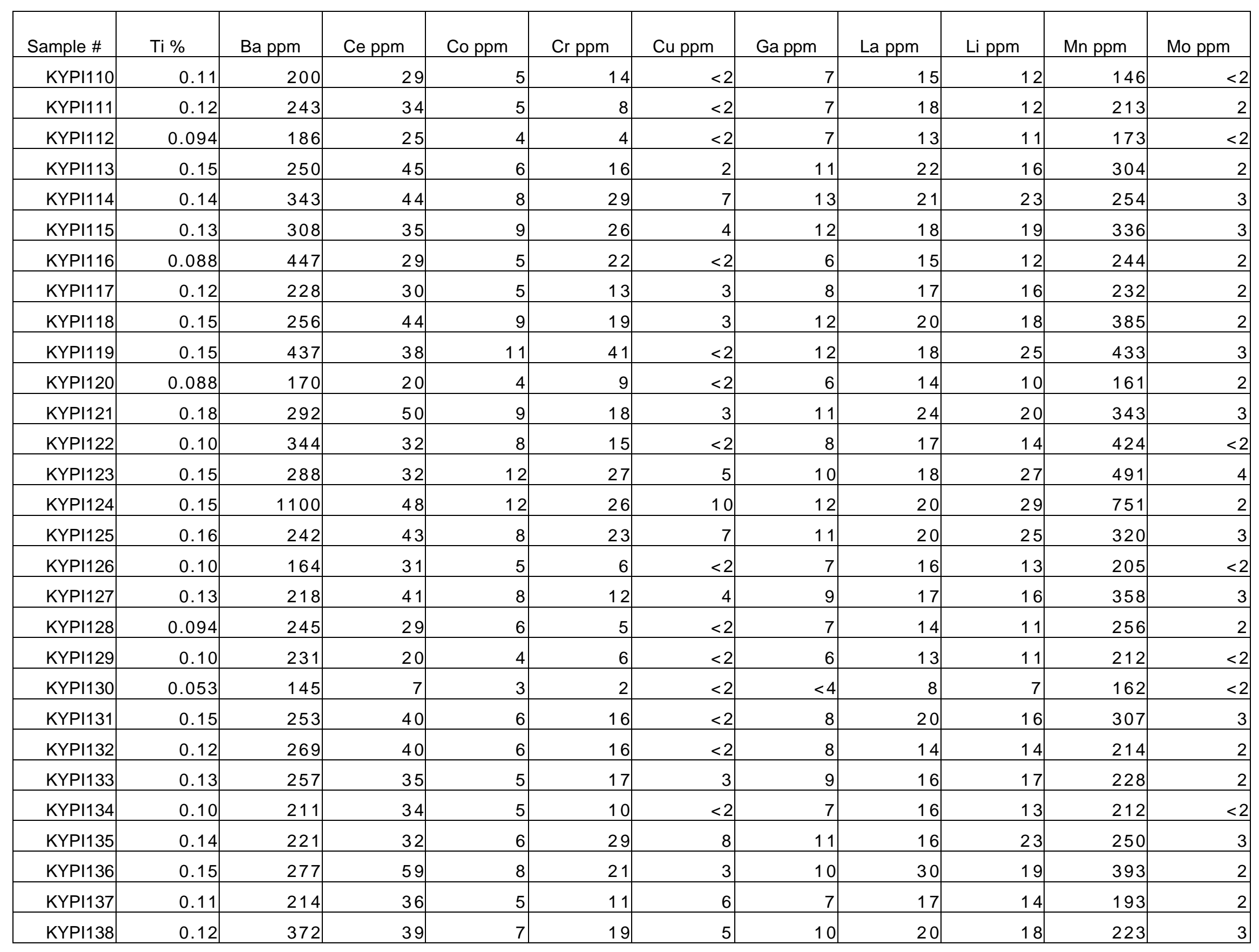




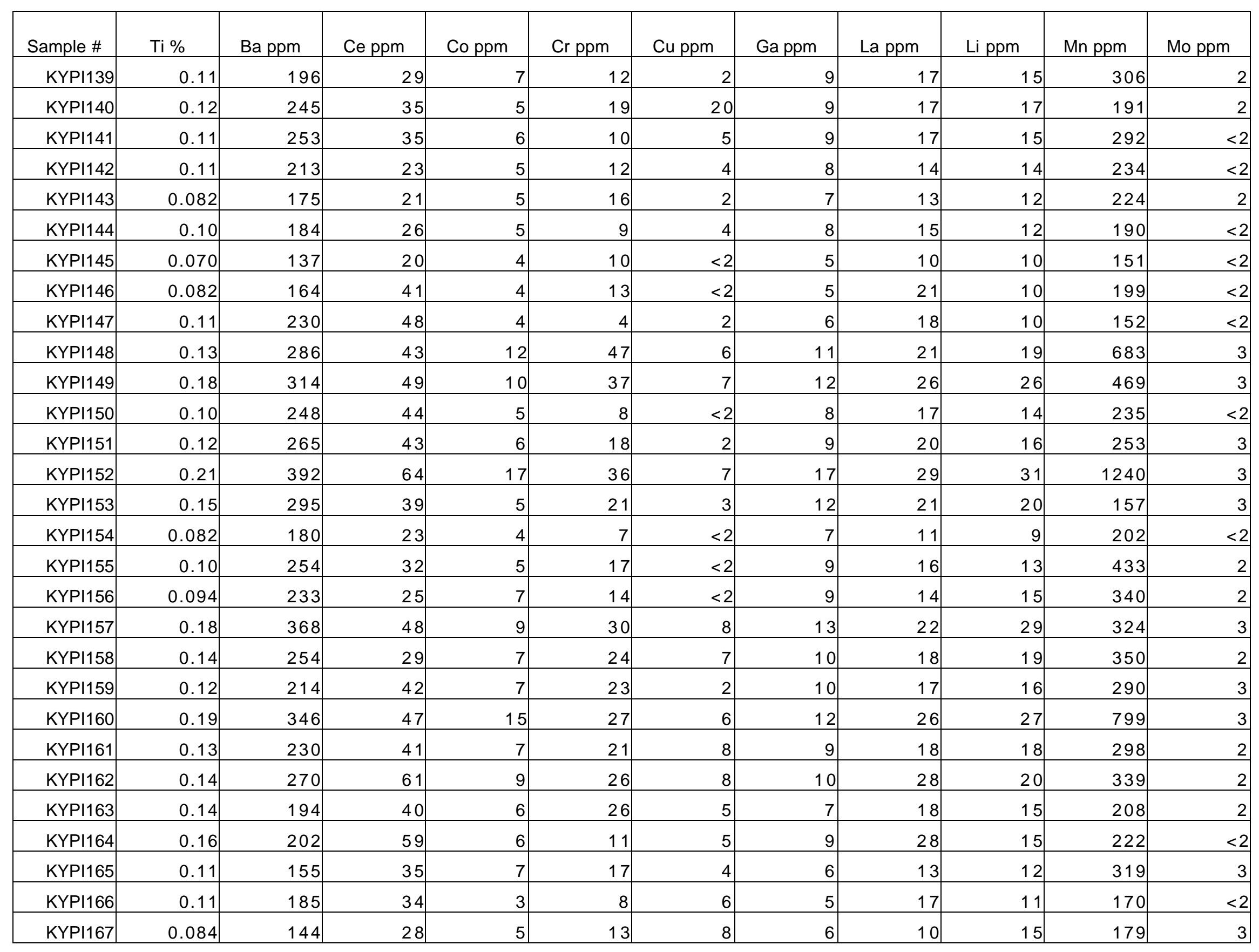




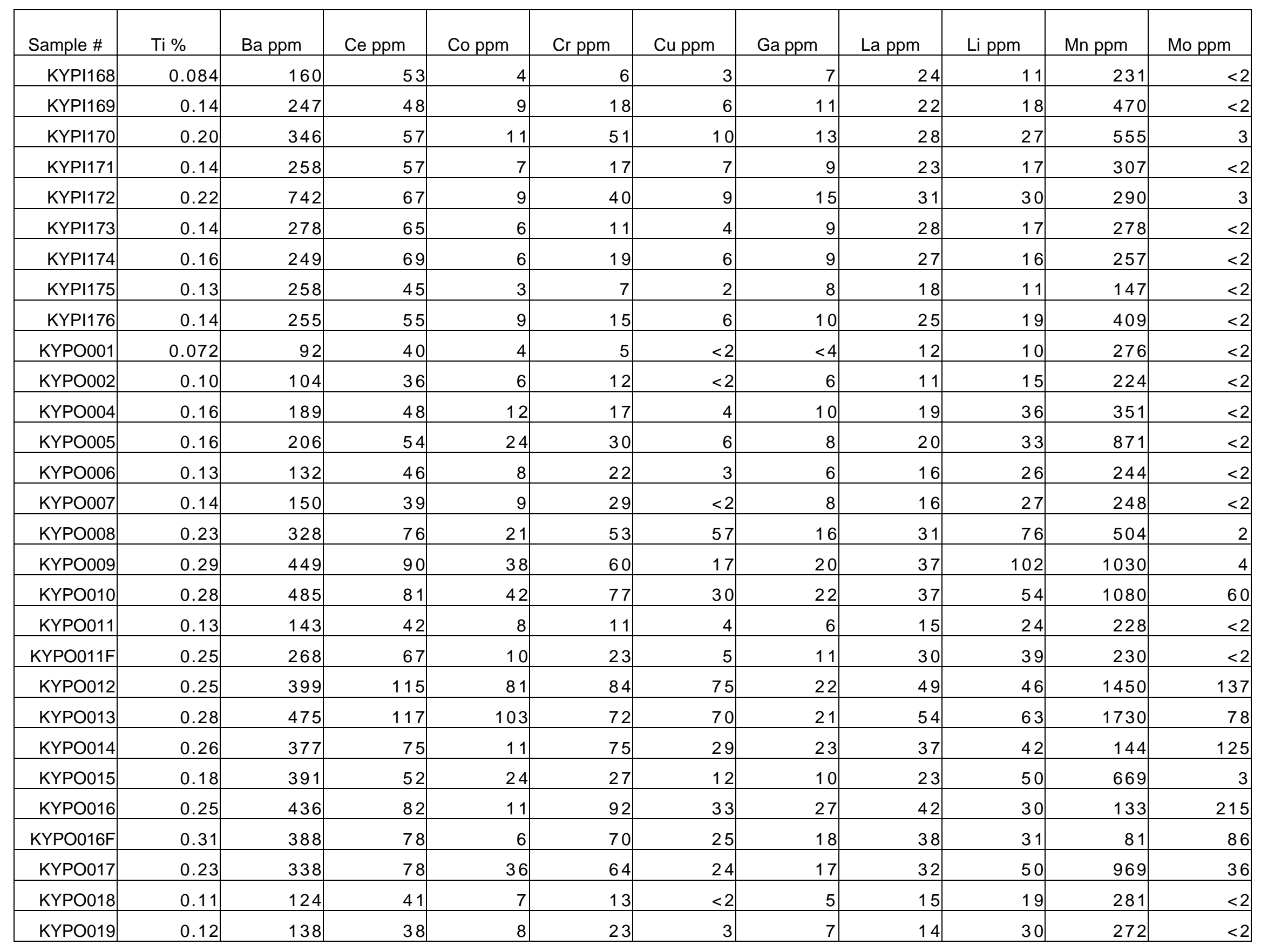




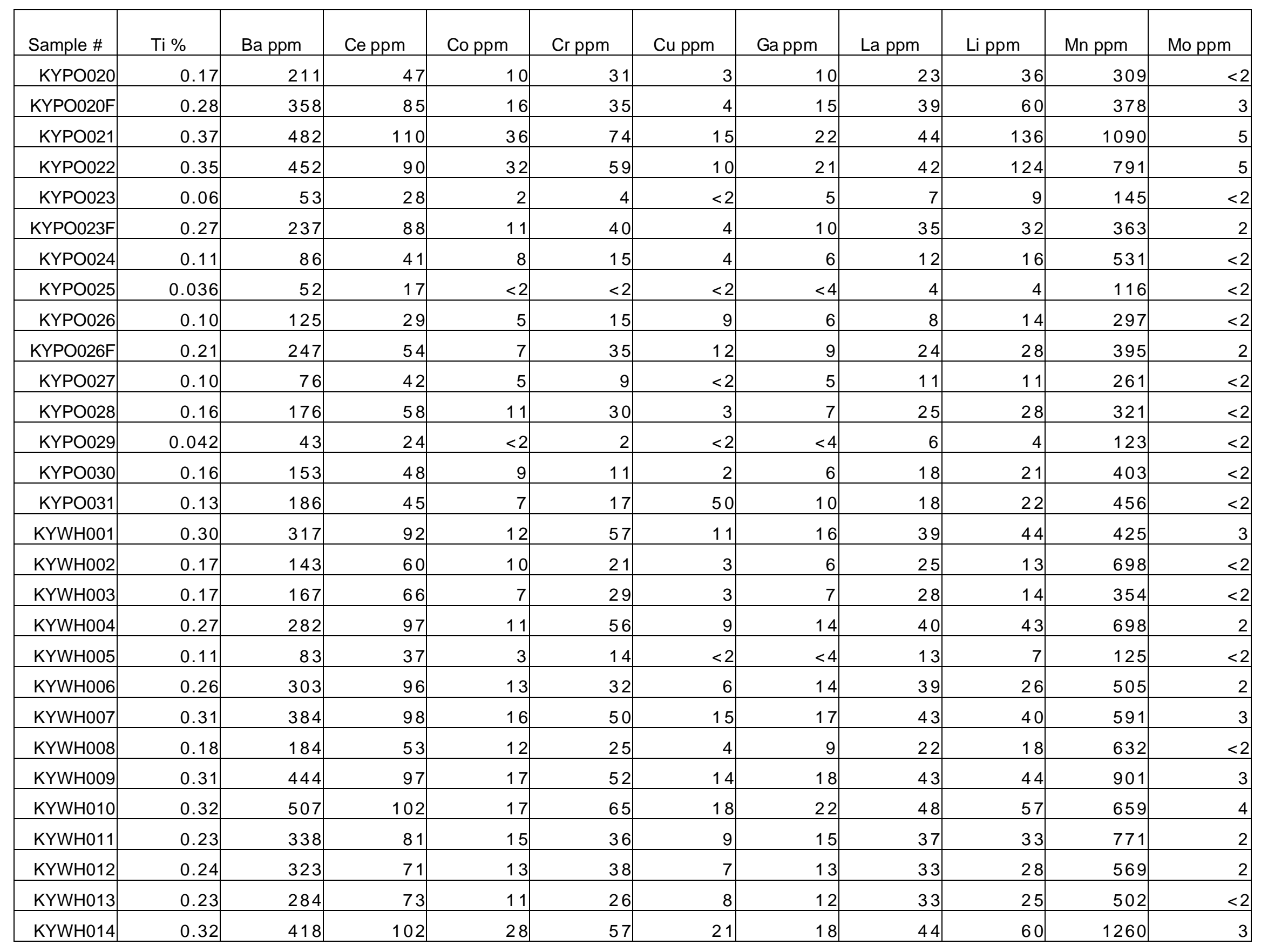




\begin{tabular}{|c|c|c|c|c|c|c|c|c|c|c|c|}
\hline Sample \# & $\mathrm{Ti} \%$ & Ba ppm & Ce ppm & Co ppm & Cr ppm & Cu ppm & Ga ppm & La ppm & Li ppm & Mn ppm & Mo ppm \\
\hline KYWH015 & 0.23 & 284 & 79 & 12 & 33 & 8 & 11 & 34 & 26 & 506 & $<2$ \\
\hline KYWH016 & 0.27 & 339 & 89 & 14 & 36 & 11 & 14 & 40 & 33 & 533 & 2 \\
\hline $\mathrm{KYWH} 018$ & 0.24 & 329 & 97 & 13 & 36 & 8 & 13 & 41 & 27 & 650 & 2 \\
\hline KYWH019 & 0.20 & 285 & 68 & 11 & 85 & 7 & 11 & 33 & 24 & 577 & 3 \\
\hline $\mathrm{KYWH} 022$ & 0.21 & 210 & 124 & 8 & 20 & 4 & 8 & 50 & 17 & 385 & $<2$ \\
\hline $\mathrm{KYWH} 023$ & 0.36 & 593 & 113 & 18 & 77 & 18 & 26 & 52 & 63 & 677 & 5 \\
\hline $\mathrm{KYWH} 024$ & 0.29 & 492 & 98 & 17 & 62 & 14 & 21 & 48 & 51 & 1190 & 3 \\
\hline KYWH025 & 0.23 & 322 & 82 & 14 & 40 & 8 & 12 & 38 & 29 & 820 & $<2$ \\
\hline KYWH026 & 0.16 & 260 & 65 & 10 & 29 & 6 & 9 & 29 & 23 & 683 & $<2$ \\
\hline $\mathrm{KYWH} 030$ & 0.28 & 344 & 90 & 16 & 55 & 10 & 13 & 37 & 29 & 774 & 2 \\
\hline $\mathrm{KYWH031}$ & 0.22 & 268 & 74 & 11 & 21 & 5 & 9 & 31 & 21 & 495 & $<2$ \\
\hline KYWH032 & 0.27 & 314 & 74 & 16 & 45 & 8 & 10 & 35 & 31 & 1380 & 2 \\
\hline $\mathrm{KYWH033}$ & 0.23 & 236 & 71 & 10 & 35 & 7 & 9 & 29 & 27 & 605 & $<2$ \\
\hline $\mathrm{KYWH} 034$ & 0.23 & 205 & 67 & 6 & 26 & 5 & 8 & 28 & 19 & 307 & $<2$ \\
\hline KYWH035 & 0.27 & 306 & 91 & 12 & 37 & 7 & 12 & 35 & 30 & 656 & 2 \\
\hline $\mathrm{KYWH} 036$ & 0.25 & 226 & 62 & 7 & 33 & 4 & 8 & 25 & 24 & 345 & $<2$ \\
\hline $\mathrm{KYWH037}$ & 0.27 & 284 & 85 & 19 & 47 & 12 & 14 & 36 & 37 & 1110 & 2 \\
\hline $\mathrm{KYWH038}$ & 0.22 & 187 & 60 & 6 & 29 & 5 & 8 & 24 & 17 & 587 & $<2$ \\
\hline KYWH039 & 0.15 & 105 & 42 & 4 & 21 & 2 & 5 & 17 & 10 & 254 & $<2$ \\
\hline
\end{tabular}




\begin{tabular}{|c|c|c|c|c|c|c|c|c|c|c|c|}
\hline Sample \# & $\mathrm{Ti} \%$ & Ba ppm & Ce ppm & Co ppm & Cr ppm & Cu ppm & Ga ppm & La ppm & Li ppm & Mn ppm & Mo ppm \\
\hline KYWO001 & 0.32 & 402 & 92 & 18 & 45 & 10 & 16 & 42 & 50 & 1100 & 4 \\
\hline KYWO002 & 0.060 & 77 & 17 & 3 & 17 & $<2$ & 5 & 6 & 10 & 159 & $<2$ \\
\hline KYWO004 & 0.10 & 133 & 42 & 4 & 8 & $<2$ & 5 & 19 & 10 & 184 & $<2$ \\
\hline KYWO005 & 0.090 & 112 & 42 & 7 & 13 & $<2$ & $<4$ & 17 & 9 & 839 & $<2$ \\
\hline KYWO007E & 0.23 & 290 & 112 & 6 & 21 & 4 & 9 & 47 & 16 & 237 & 2 \\
\hline KYWO008 & 0.13 & 178 & 58 & 4 & 12 & 2 & 6 & 20 & 10 & 240 & $<2$ \\
\hline KYWO009 & 0.12 & 181 & 83 & 4 & 13 & $<2$ & 6 & 30 & 8 & 157 & $<2$ \\
\hline KYWO010 & 0.11 & 157 & 68 & 2 & 9 & $<2$ & 5 & 27 & 7 & 232 & $<2$ \\
\hline KYWO011 & 0.090 & 150 & 40 & 4 & 9 & $<2$ & 5 & 13 & 7 & 236 & $<2$ \\
\hline KYWO015 & 0.15 & 192 & 52 & 16 & 33 & 5 & 9 & 20 & 23 & 894 & 2 \\
\hline KYWO015E & 0.22 & 238 & 75 & 13 & 38 & 6 & 9 & 32 & 27 & 785 & 3 \\
\hline KYWO016 & 0.23 & 271 & 71 & 20 & 39 & 4 & 12 & 29 & 28 & 1360 & 3 \\
\hline KYWO017 & 0.17 & 215 & 70 & 7 & 22 & 3 & 7 & 31 & 15 & 376 & $<2$ \\
\hline KYWO018 & 0.13 & 194 & 49 & 7 & 17 & 3 & 7 & 18 & 14 & 346 & $<2$ \\
\hline KYWO019 & 0.14 & 182 & 39 & 6 & 15 & 2 & 6 & 20 & 14 & 234 & $<2$ \\
\hline KYWO019E & 0.19 & 235 & 76 & 8 & 27 & 4 & 9 & 32 & 20 & 302 & $<2$ \\
\hline KYWO020 & 0.16 & 183 & 55 & 7 & 15 & 3 & 8 & 22 & 16 & 332 & $<2$ \\
\hline KYWO021 & 0.14 & 174 & 48 & 7 & 19 & 3 & 8 & 17 & 15 & 291 & $<2$ \\
\hline KYWO022 & 0.14 & 186 & 47 & 7 & 21 & 4 & 7 & 17 & 15 & 281 & $<2$ \\
\hline
\end{tabular}




\begin{tabular}{|c|c|c|c|c|c|c|c|c|c|c|c|}
\hline Sample \# & $\mathrm{Ti} \%$ & Ba ppm & Ce ppm & Co ppm & Cr ppm & Cu ppm & Ga ppm & La ppm & Li ppm & Mn ppm & Mo ppm \\
\hline KYWO027 & 0.13 & 119 & 33 & 7 & 17 & $<2$ & 5 & 13 & 14 & 309 & $<2$ \\
\hline KYWO028 & 0.14 & 185 & 70 & 15 & 19 & 3 & 11 & 25 & 24 & 617 & 2 \\
\hline KYWO029 & 0.12 & 180 & 50 & 10 & 17 & 2 & 9 & 18 & 17 & 600 & $<2$ \\
\hline KYWO031 & 0.090 & 95 & 38 & 4 & 19 & 2 & $<4$ & 14 & 9 & 161 & $<2$ \\
\hline KYWO031E & 0.084 & 128 & 49 & 5 & 11 & $<2$ & 5 & 18 & 12 & 198 & $<2$ \\
\hline KYWO032 & 0.10 & 125 & 38 & 6 & 17 & 4 & 6 & 13 & 12 & 250 & $<2$ \\
\hline KYWO033 & 0.15 & 190 & 58 & 10 & 23 & 3 & 7 & 26 & 18 & 414 & 2 \\
\hline KYWO034 & 0.14 & 220 & 55 & 10 & 24 & 3 & 8 & 21 & 26 & 644 & $<2$ \\
\hline KYWO034E & 0.22 & 295 & 60 & 13 & 29 & 5 & 11 & 31 & 35 & 703 & 4 \\
\hline KYWO035 & 0.19 & 260 & 65 & 13 & 35 & 7 & 11 & 28 & 28 & 599 & 3 \\
\hline KYWO036 & 0.17 & 257 & 69 & 16 & 31 & 5 & 12 & 26 & 27 & 880 & $<2$ \\
\hline KYWO037 & 0.12 & 217 & 53 & 5 & 17 & 3 & 6 & 21 & 12 & 318 & $<2$ \\
\hline KYWO039 & 0.078 & 139 & 38 & 3 & 10 & $<2$ & 5 & 13 & 9 & 211 & $<2$ \\
\hline KYWO040 & 0.11 & 163 & 43 & 6 & 13 & 2 & 6 & 18 & 11 & 406 & $<2$ \\
\hline KYWO041 & 0.10 & 176 & 61 & 6 & 9 & 2 & 7 & 23 & 12 & 349 & $<2$ \\
\hline KYWO042 & 0.11 & 186 & 73 & 5 & 14 & 2 & 5 & 30 & 10 & 284 & $<2$ \\
\hline KYWO045 & 0.15 & 200 & 54 & 11 & 23 & 4 & 9 & 22 & 19 & 564 & $<2$ \\
\hline KYWO046 & 0.21 & 254 & 59 & 15 & 35 & 5 & 12 & 24 & 29 & 643 & 3 \\
\hline KYWO047 & 0.13 & 181 & 50 & 8 & 13 & 3 & 8 & 19 & 16 & 372 & 2 \\
\hline KYWO048 & 0.26 & 293 & 61 & 10 & 22 & 3 & 7 & 29 & 19 & 169 & $<2$ \\
\hline KYWO049 & 0.048 & 64 & 22 & 2 & 6 & $<2$ & $<4$ & 8 & 9 & 128 & $<2$ \\
\hline KYWO050 & 0.048 & 58 & 25 & $<2$ & 5 & $<2$ & $<4$ & 12 & 5 & 159 & $<2$ \\
\hline KYWO051 & 0.32 & 451 & 120 & 29 & 61 & 18 & 20 & 44 & 62 & 1650 & 4 \\
\hline KYWO052 & 0.10 & 127 & 26 & 8 & 19 & $<2$ & 5 & 12 & 13 & 467 & $<2$ \\
\hline KYWO053 & 0.42 & 510 & 100 & 21 & 71 & 16 & 29 & 48 & 104 & 677 & 6 \\
\hline
\end{tabular}




\begin{tabular}{|c|c|c|c|c|c|c|c|c|c|c|c|}
\hline KYBE002E & 13 & 45 & 14 & 19 & 7 & 52 & 11 & 50 & 17 & 2 & 54 \\
\hline KYBE004E & 17 & 48 & 33 & 30 & 12 & 104 & 11 & 81 & 24 & 2 & 103 \\
\hline KYBE005E & 18 & 32 & 13 & 17 & 10 & 61 & 9 & 64 & 19 & 2 & 48 \\
\hline KYBE008E & 14 & 33 & 11 & 15 & 6 & 42 & 6 & 45 & 15 & 2 & 43 \\
\hline KYBE009E & 14 & 32 & 15 & 28 & 7 & 69 & 9 & 57 & 15 & 2 & 68 \\
\hline KYBE010E & 12 & 30 & 11 & 16 & 6 & 48 & 7 & 46 & 15 & 2 & 48 \\
\hline KYBE011E & 18 & 40 & 18 & 22 & 11 & 74 & 8 & 75 & 21 & 3 & 81 \\
\hline KYBE012E & 7 & 18 & 10 & 17 & 4 & 21 & $<6$ & 29 & 14 & 1 & 33 \\
\hline KYBE016E & 18 & 29 & 20 & 23 & 9 & 65 & $<6$ & 69 & 17 & 2 & 74 \\
\hline KYBE017E & 16 & 30 & 20 & 18 & 8 & 54 & 8 & 58 & 14 & 2 & 62 \\
\hline KYBE018E & 17 & 40 & 18 & 17 & 9 & 57 & 9 & 61 & 18 & 3 & 60 \\
\hline KYBE019E & 5 & 18 & 8 & 9 & 2 & 14 & $<6$ & 17 & 6 & $<1$ & 28 \\
\hline KYBE020E & 7 & 28 & 12 & 10 & 3 & 17 & $<6$ & 22 & 8 & 1 & 50 \\
\hline KYBE021E & 14 & 29 & 14 & 17 & 7 & 47 & $<6$ & 52 & 15 & 2 & 56 \\
\hline KYBE022E & 17 & 35 & 25 & 23 & 10 & 68 & 13 & 74 & 19 & 2 & 86 \\
\hline KYBE023E & 11 & 40 & 23 & 22 & 10 & 116 & 8 & 74 & 17 & 2 & 76 \\
\hline KYBE024E & 17 & 40 & 20 & 22 & 10 & 67 & 11 & 72 & 21 & 3 & 67 \\
\hline KYBE025E & 14 & 32 & 16 & 22 & 8 & 54 & $<6$ & 60 & 16 & 2 & 60 \\
\hline
\end{tabular}




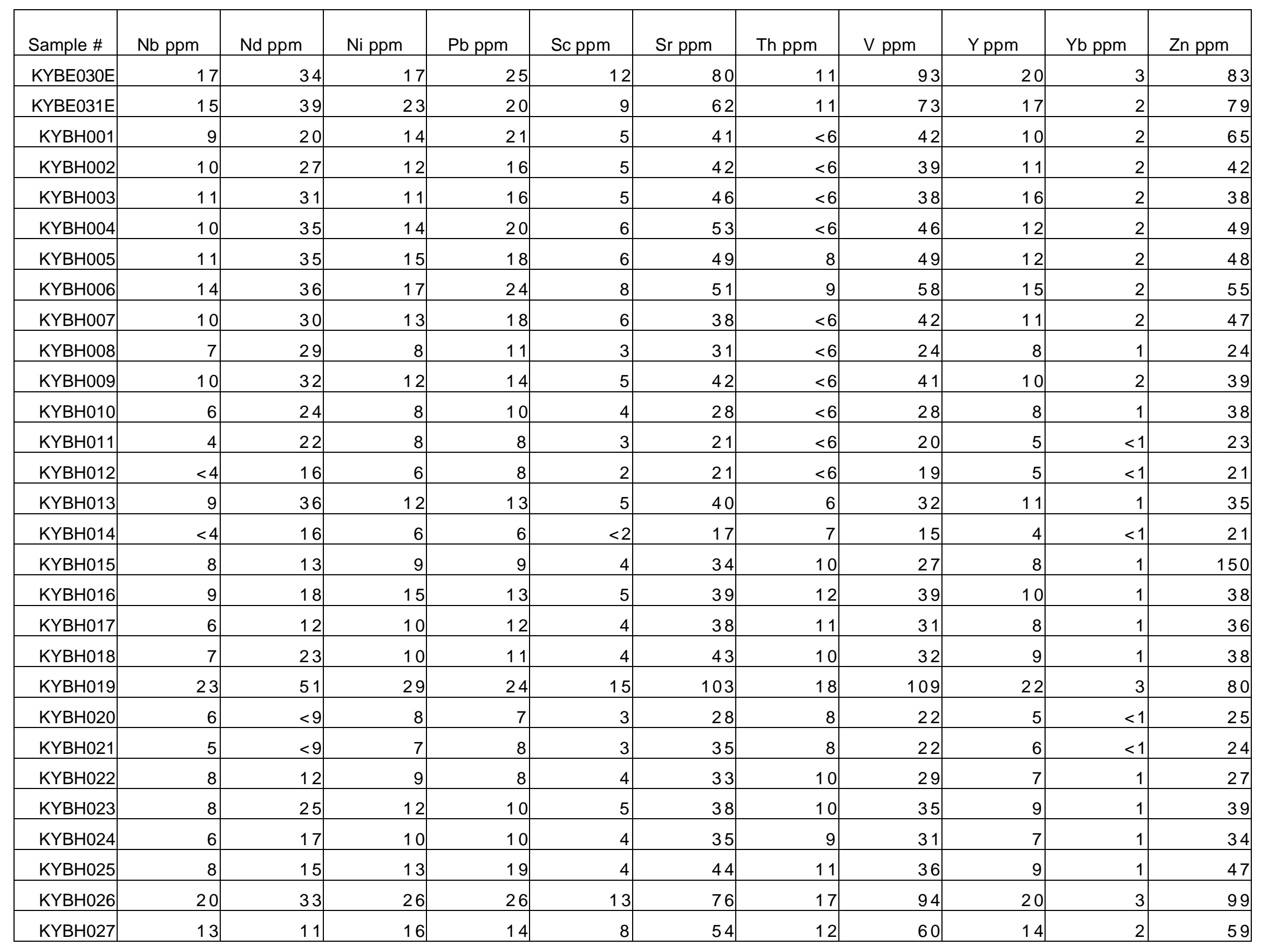




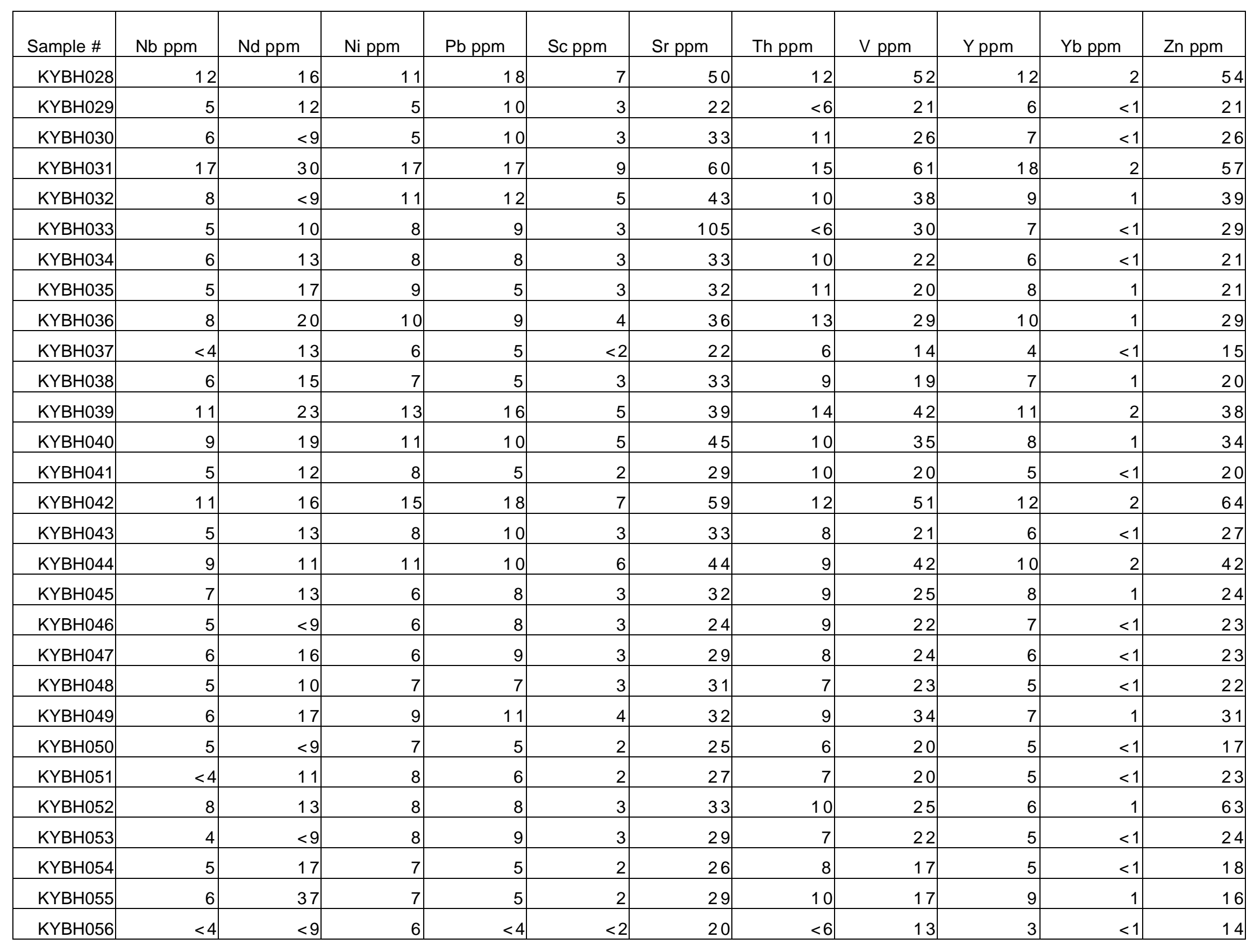




\begin{tabular}{|c|c|c|c|c|c|c|c|c|c|c|c|}
\hline KYBH058 & 5 & 11 & 8 & 5 & 2 & 27 & 8 & 15 & 5 & $<1$ & 17 \\
\hline KYBH060 & $<4$ & 12 & 8 & $<4$ & $<2$ & 23 & 6 & 15 & 4 & $<1$ & 17 \\
\hline KYBH061 & 6 & 22 & 17 & 8 & 3 & 32 & 9 & 26 & 8 & 1 & 37 \\
\hline KYBH064 & 10 & 19 & 11 & 13 & 5 & 51 & 9 & 40 & 10 & 2 & 35 \\
\hline KYBH065 & 7 & 17 & 17 & 8 & 3 & 33 & 8 & 25 & 7 & 1 & 34 \\
\hline KYBH066 & 5 & 11 & 7 & 7 & 3 & 35 & 14 & 22 & 6 & 1 & 24 \\
\hline KYBH067 & 9 & 17 & 10 & 11 & 4 & 43 & 10 & 34 & 8 & 1 & 34 \\
\hline KYBH068 & 7 & 18 & 9 & 8 & 3 & 40 & 11 & 23 & 7 & 1 & 24 \\
\hline KYBH072 & 6 & 14 & 9 & 6 & 3 & 30 & 9 & 24 & 7 & 1 & 36 \\
\hline KYBH073 & 9 & 19 & 13 & 10 & 5 & 43 & 9 & 37 & 9 & 1 & 45 \\
\hline KYBH074 & 6 & $<9$ & 10 & 9 & 3 & 34 & 8 & 28 & 6 & 1 & 30 \\
\hline KYBH075 & 8 & 17 & 12 & 12 & 5 & 38 & 8 & 39 & 8 & 1 & 45 \\
\hline KYBH076 & 12 & 22 & 15 & 21 & 6 & 60 & 12 & 52 & 12 & 2 & 80 \\
\hline $\mathrm{KYBH} 077 \mathrm{~F}$ & 13 & 30 & 13 & 25 & 8 & 73 & 9 & 58 & 16 & 2 & 53 \\
\hline $\mathrm{KYBH} 078 \mathrm{~F}$ & 15 & 43 & 15 & 23 & 9 & 67 & 14 & 58 & 19 & 3 & 58 \\
\hline $\mathrm{KYBH} 079 \mathrm{~F}$ & 14 & 43 & 13 & 17 & 8 & 63 & 10 & 51 & 17 & 2 & 49 \\
\hline $\mathrm{KYBH} 080 \mathrm{~F}$ & 17 & 44 & 19 & 25 & 9 & 73 & 8 & 64 & 20 & 3 & 86 \\
\hline KYBH081F & 12 & 42 & 12 & 19 & 7 & 62 & 9 & 51 & 16 & 2 & 69 \\
\hline
\end{tabular}




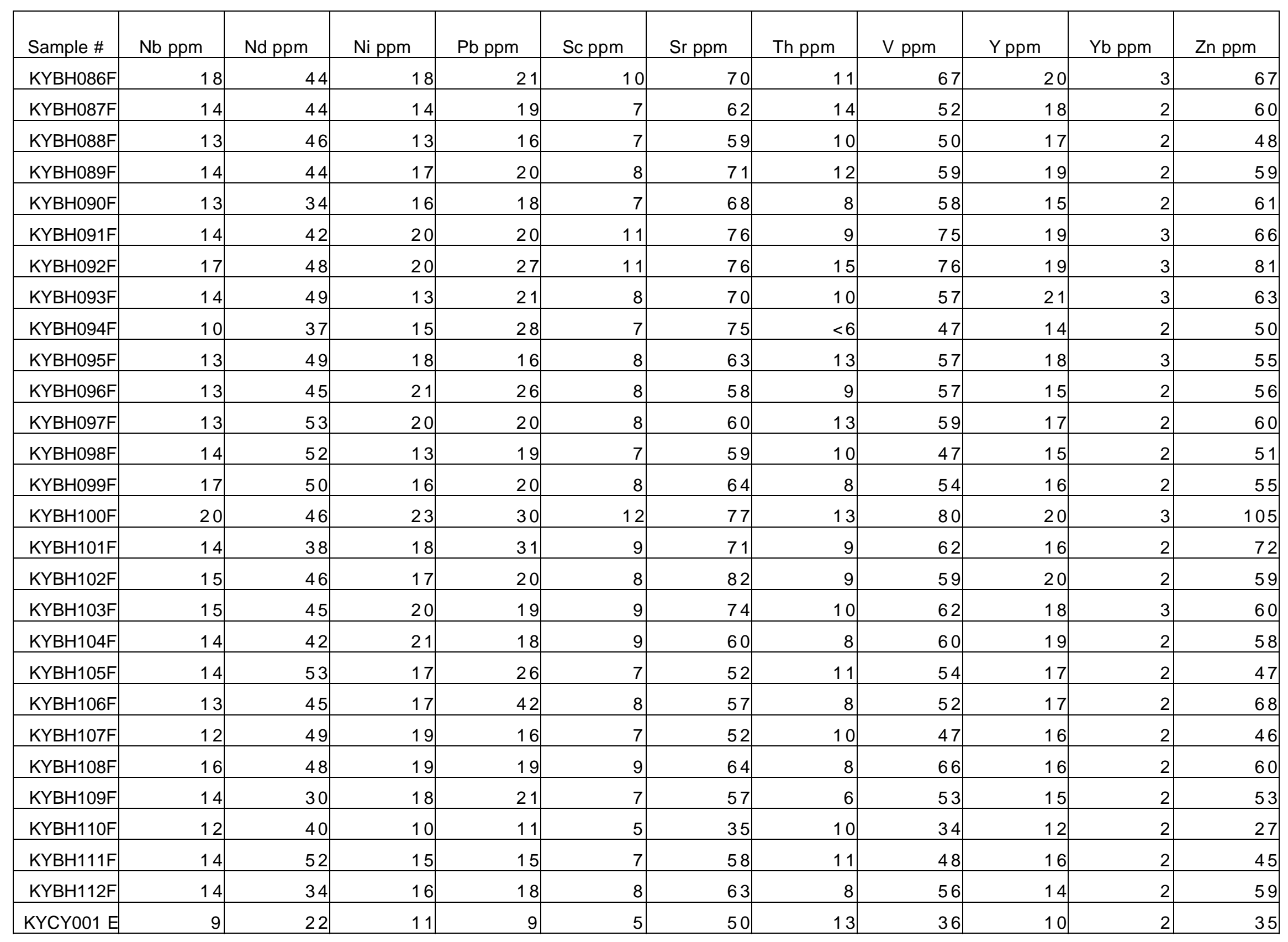




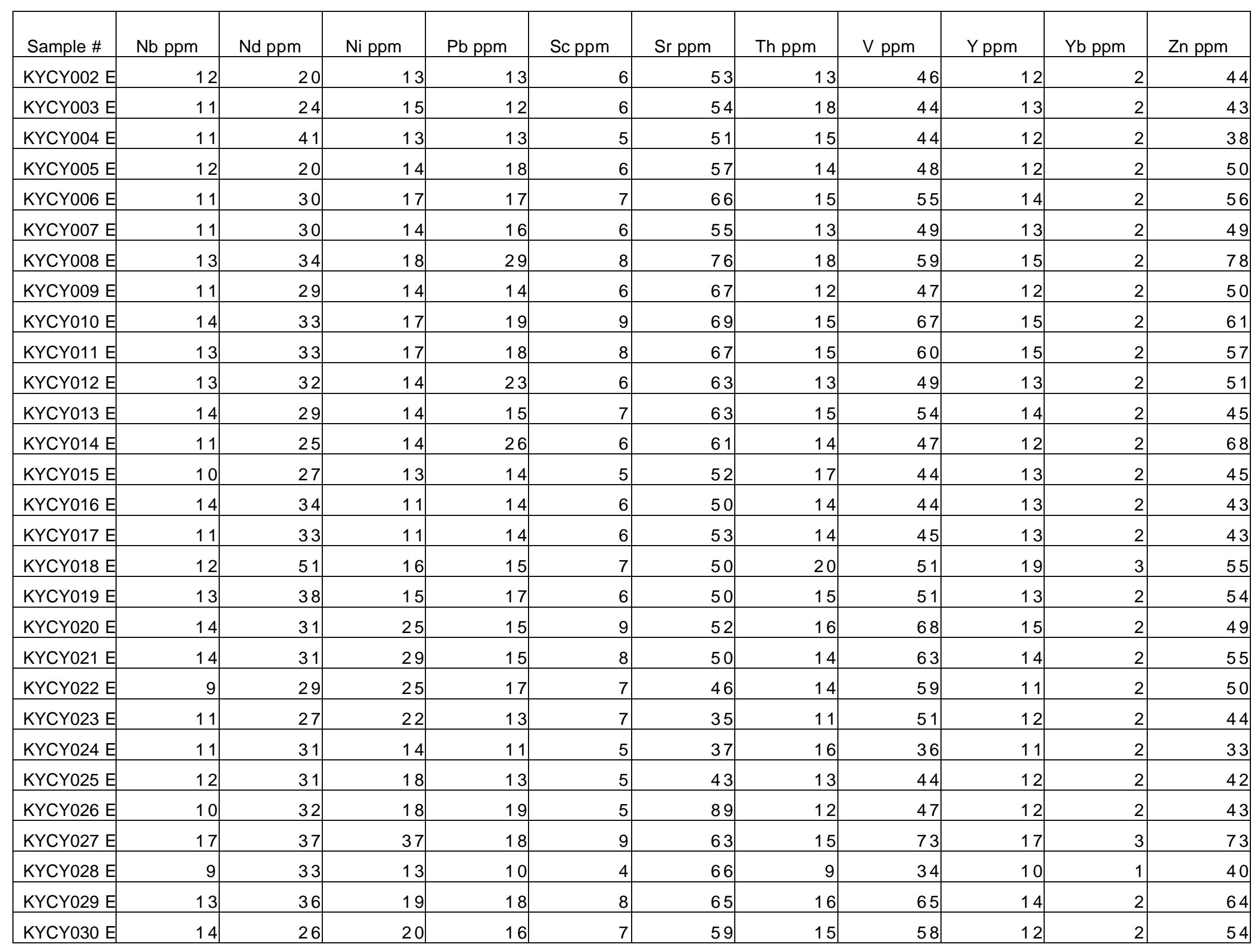




\begin{tabular}{|c|c|c|c|c|c|c|c|c|c|c|c|}
\hline Sample \# & $\mathrm{Nb}$ ppm & Nd ppm & Ni ppm & $\mathrm{Pb} \mathrm{ppm}$ & Sc ppm & Sr ppm & Th ppm & $\mathrm{V}$ ppm & Y ppm & Yb ppm & Zn ppm \\
\hline KYCY032 E & 12 & 36 & 15 & 15 & 6 & 55 & 12 & 45 & 12 & 2 & 55 \\
\hline KYCY034 E & 10 & 33 & 16 & 11 & 5 & 39 & 12 & 41 & 11 & 2 & 43 \\
\hline KYCY035 E & 12 & 25 & 20 & 11 & 5 & 39 & 8 & 40 & 12 & 2 & 45 \\
\hline KYCY038 E & 13 & 27 & 17 & 12 & 7 & 45 & 15 & 54 & 15 & 2 & 48 \\
\hline KYCY039 E & 8 & 21 & 13 & 11 & 4 & 31 & 10 & 31 & 9 & 1 & 29 \\
\hline KYCY040 E & 10 & 18 & 15 & 8 & 4 & 30 & 12 & 32 & 10 & 2 & 29 \\
\hline KYCY041 E & 8 & 28 & 13 & 7 & 4 & 30 & 12 & 29 & 10 & 2 & 30 \\
\hline KYCY042 E & 15 & 42 & 22 & 18 & 8 & 62 & 9 & 65 & 13 & 2 & 76 \\
\hline KYES003 & 11 & 26 & 32 & 25 & 9 & 43 & 6 & 101 & 11 & 2 & 82 \\
\hline KYES004 & 8 & 16 & 25 & 20 & 6 & 40 & 9 & 78 & 11 & 1 & 79 \\
\hline KYES005 & 6 & 21 & 17 & 15 & 4 & 26 & $<6$ & 47 & 8 & 1 & 48 \\
\hline KYES006 & 7 & 22 & 13 & 33 & 4 & 22 & $<6$ & 38 & 8 & 1 & 35 \\
\hline KYES007 & $<4$ & 12 & 9 & 7 & 2 & 14 & $<6$ & 21 & 6 & $<1$ & 25 \\
\hline KYES017F & 12 & 24 & 48 & 19 & 7 & 44 & 9 & 112 & 17 & 2 & 138 \\
\hline KYES024F & 16 & 24 & 38 & 19 & 8 & 45 & 13 & 84 & 15 & 2 & 130 \\
\hline KYES030F & 6 & 39 & 67 & 28 & 8 & 62 & 11 & 105 & 26 & 2 & 136 \\
\hline KYES034 & 8 & 34 & 21 & 15 & 5 & 35 & 11 & 64 & 9 & 2 & 53 \\
\hline KYES036 & 11 & 19 & 22 & 36 & 6 & 42 & 6 & 72 & 11 & 2 & 64 \\
\hline
\end{tabular}




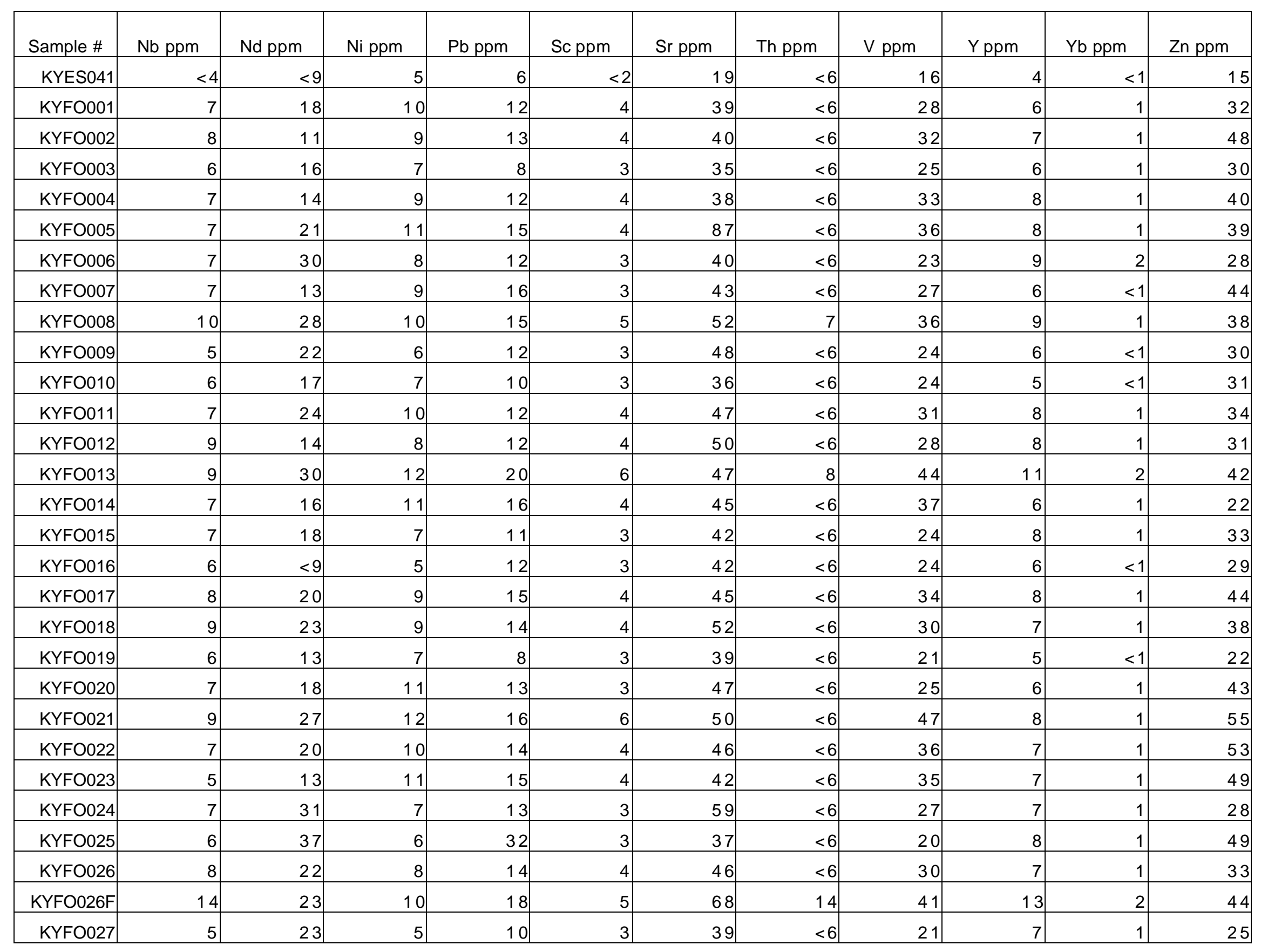




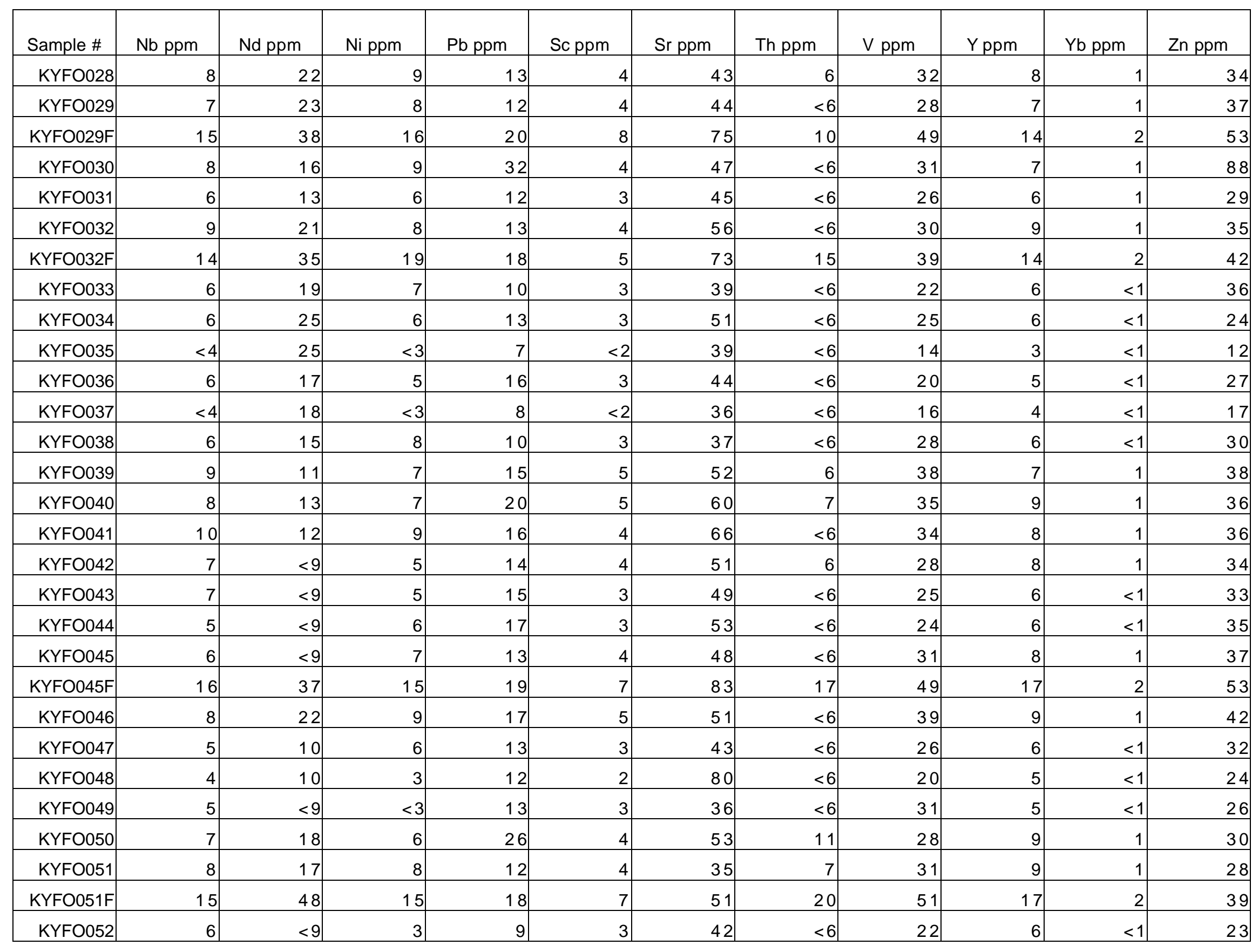




\begin{tabular}{|c|c|c|c|c|c|c|c|c|c|c|c|}
\hline KYFO054 & 6 & 18 & 6 & 12 & 3 & 34 & $<6$ & 22 & 8 & 1 & 27 \\
\hline KYFO056 & 9 & 28 & 11 & 15 & 5 & 47 & $<6$ & 41 & 8 & 1 & 39 \\
\hline KYFO057 & 8 & 14 & 9 & 16 & 5 & 47 & 8 & 36 & 7 & 1 & 38 \\
\hline KYFO061 & 6 & $<9$ & 7 & 11 & 3 & 33 & 8 & 25 & 6 & $<1$ & 23 \\
\hline KYFO062 & 6 & $<9$ & 7 & 12 & 3 & 49 & 7 & 24 & 5 & $<1$ & 35 \\
\hline KYFO063 & 5 & 10 & 9 & 14 & 3 & 41 & 8 & 24 & 5 & $<1$ & 29 \\
\hline KYFO064 & 12 & 19 & 18 & 20 & 9 & 80 & 11 & 66 & 10 & 2 & 56 \\
\hline KYFO065 & 9 & $<9$ & 17 & 81 & 6 & 52 & 8 & 46 & 8 & 1 & 45 \\
\hline KYFO070 & 8 & 16 & 9 & 17 & 4 & 62 & 8 & 33 & 7 & 1 & 34 \\
\hline KYFO071 & 8 & $<9$ & 10 & 14 & 4 & 51 & 8 & 33 & 8 & 1 & 32 \\
\hline KYFO072 & 5 & $<9$ & 7 & 10 & 3 & 33 & $<6$ & 22 & 5 & $<1$ & 26 \\
\hline KYFO073 & 10 & 19 & 20 & 19 & 6 & 56 & 9 & 44 & 10 & 2 & 54 \\
\hline KYFO074 & 6 & 21 & 22 & 22 & 4 & 54 & 8 & 30 & 7 & 1 & 71 \\
\hline KYFO075 & 12 & 20 & 15 & 16 & 7 & 63 & 14 & 55 & 10 & 2 & 38 \\
\hline KYFO076 & 5 & $<9$ & 6 & 12 & 2 & 26 & $<6$ & 18 & 4 & $<1$ & 25 \\
\hline KYFO077 & $<4$ & $<9$ & $<3$ & 11 & $<2$ & 25 & $<6$ & 14 & 4 & $<1$ & 14 \\
\hline KYFO078 & 5 & $<9$ & 4 & 14 & 2 & 29 & $<6$ & 18 & 5 & $<1$ & 20 \\
\hline KYFO079 & $<4$ & $<9$ & 5 & 8 & $<2$ & 24 & 7 & 12 & 3 & $<1$ & 13 \\
\hline
\end{tabular}




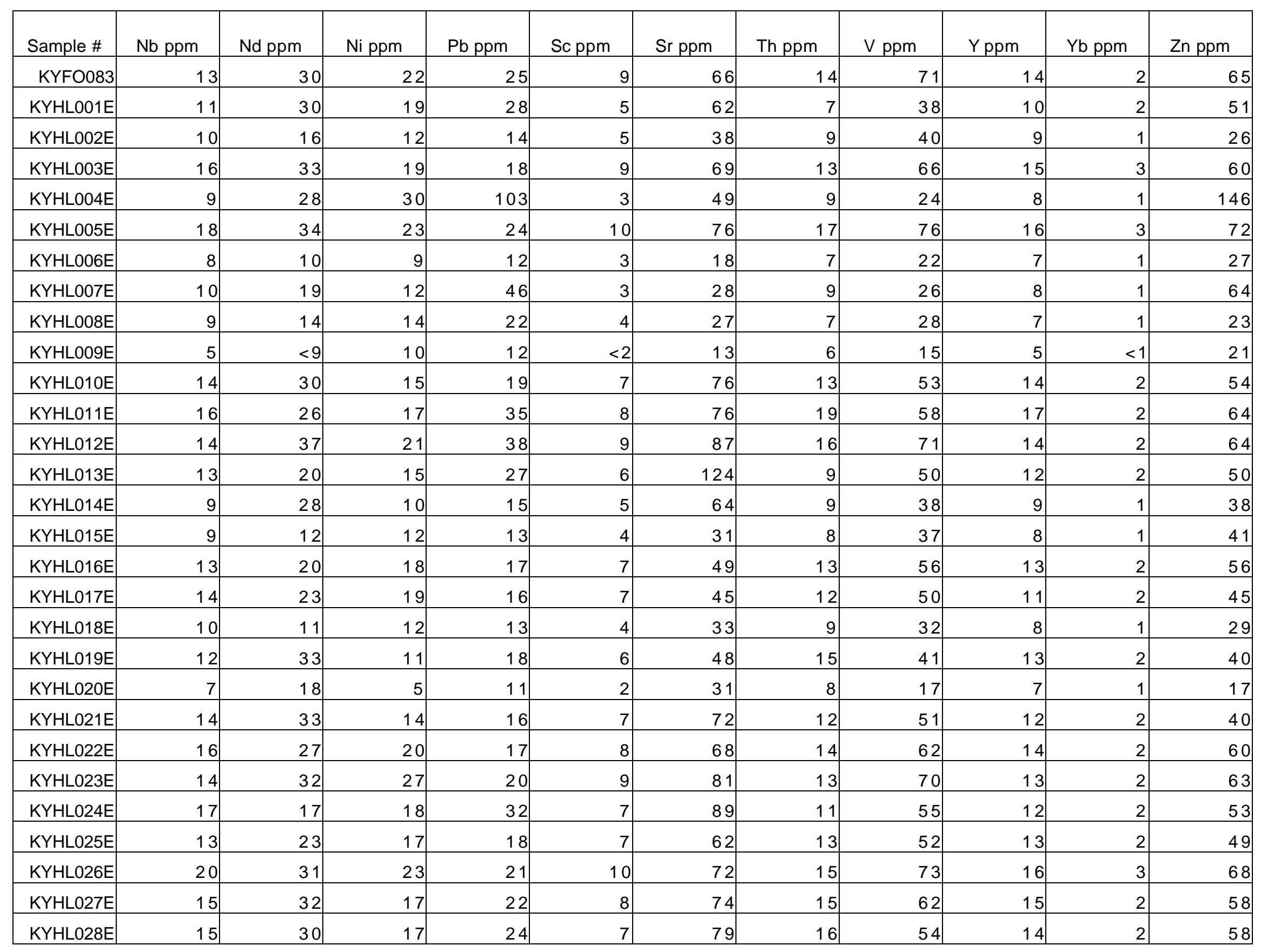




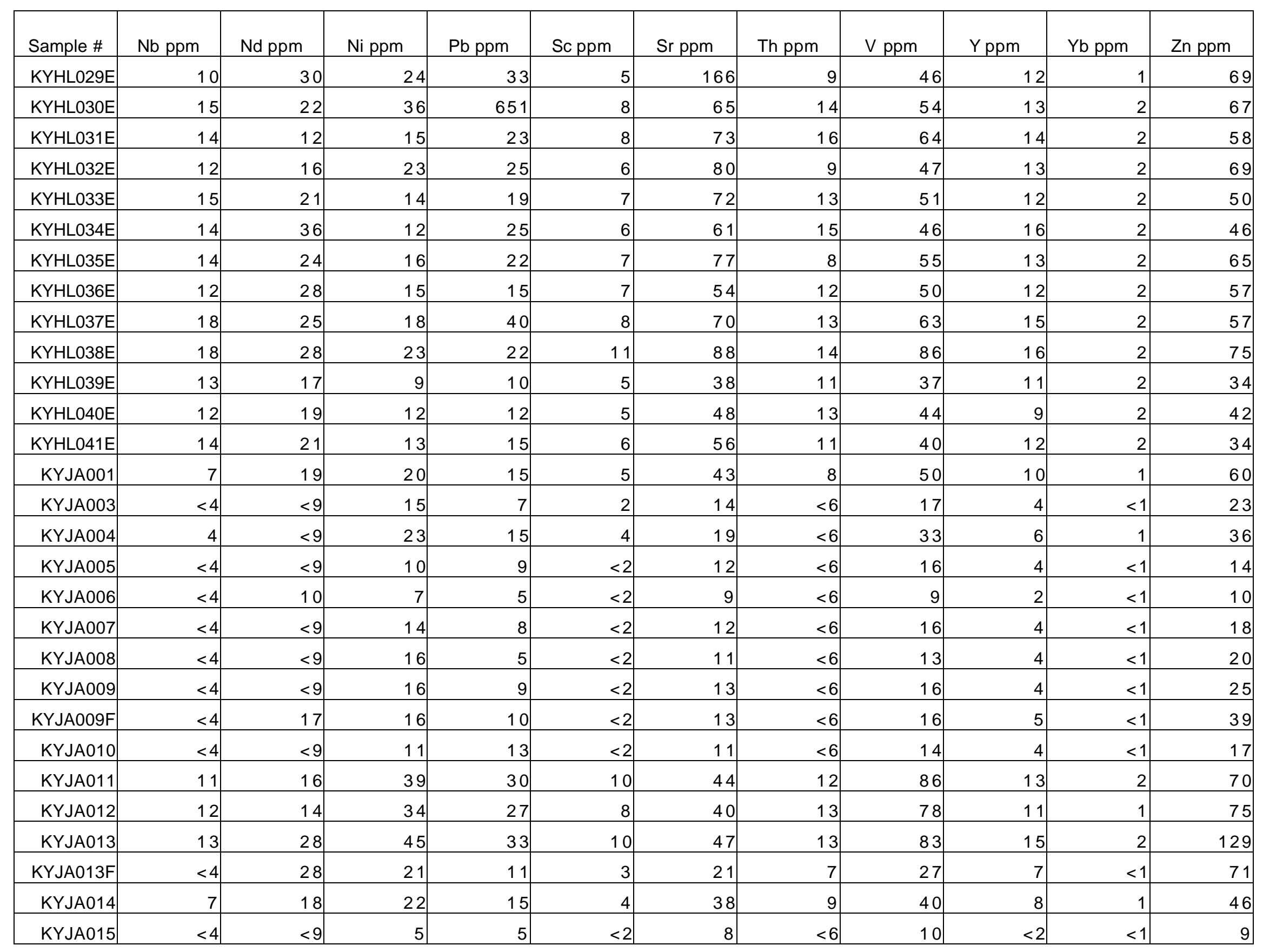




\begin{tabular}{|c|c|c|c|c|c|c|c|c|c|c|c|}
\hline Sample \# & $\mathrm{Nb}$ ppm & Nd ppm & Ni ppm & $\mathrm{Pb} \mathrm{ppm}$ & Sc ppm & Sr ppm & Th ppm & $\mathrm{V}$ ppm & Y ppm & Yb ppm & Zn ppm \\
\hline KYJA017 & $<4$ & $<9$ & 18 & 10 & 2 & 13 & $<6$ & 21 & 4 & $<1$ & 25 \\
\hline KYJA018F & $<4$ & 32 & 9 & 5 & $<2$ & 11 & $<6$ & 12 & 4 & $<1$ & 22 \\
\hline KYJA019 & $<4$ & $<9$ & 8 & 6 & $<2$ & 14 & $<6$ & 9 & 2 & $<1$ & 10 \\
\hline KYJA022 & 5 & $<9$ & 26 & 11 & 3 & 22 & $<6$ & 34 & 6 & 1 & 41 \\
\hline KYJA023 & 6 & 11 & 18 & 17 & 5 & 23 & $<6$ & 65 & 8 & 1 & 58 \\
\hline KYJA023F & $<4$ & 18 & 7 & 6 & $<2$ & 11 & $<6$ & 17 & 3 & $<1$ & 21 \\
\hline KYJA024 & 7 & 11 & 30 & 28 & 6 & 38 & 13 & 58 & 10 & 1 & 65 \\
\hline KYJA025 & 11 & 15 & 31 & 27 & 8 & 44 & 10 & 69 & 12 & 2 & 59 \\
\hline KYJA029 & 8 & 10 & 14 & 12 & 5 & 24 & 9 & 36 & 9 & 1 & 21 \\
\hline KYJA030 & 7 & $<9$ & 11 & 12 & 4 & 23 & 10 & 31 & 6 & 1 & 31 \\
\hline KYJA031 & $<4$ & $<9$ & 18 & 8 & $<2$ & 17 & $<6$ & 17 & 4 & $<1$ & 29 \\
\hline KYJA032 & $<4$ & $<9$ & 8 & 5 & $<2$ & 10 & $<6$ & 13 & 3 & $<1$ & 15 \\
\hline KYJA033 & $<4$ & $<9$ & 34 & 8 & 2 & 13 & 6 & 20 & 9 & $<1$ & 42 \\
\hline KYJA034 & 5 & 10 & 25 & 26 & 5 & 44 & 9 & 45 & 9 & 1 & 50 \\
\hline KYJA034F & $<4$ & 23 & 9 & 15 & $<2$ & 15 & $<6$ & 15 & 4 & $<1$ & 39 \\
\hline KYJA035 & $<4$ & $<9$ & 11 & 17 & 3 & 15 & 7 & 35 & 8 & 1 & 30 \\
\hline KYJA036 & 8 & $<9$ & 20 & 19 & 5 & 32 & 7 & 46 & 10 & 2 & 39 \\
\hline KYJA037F & 7 & 14 & 11 & 10 & 3 & 20 & 9 & 23 & 10 & 1 & 18 \\
\hline
\end{tabular}




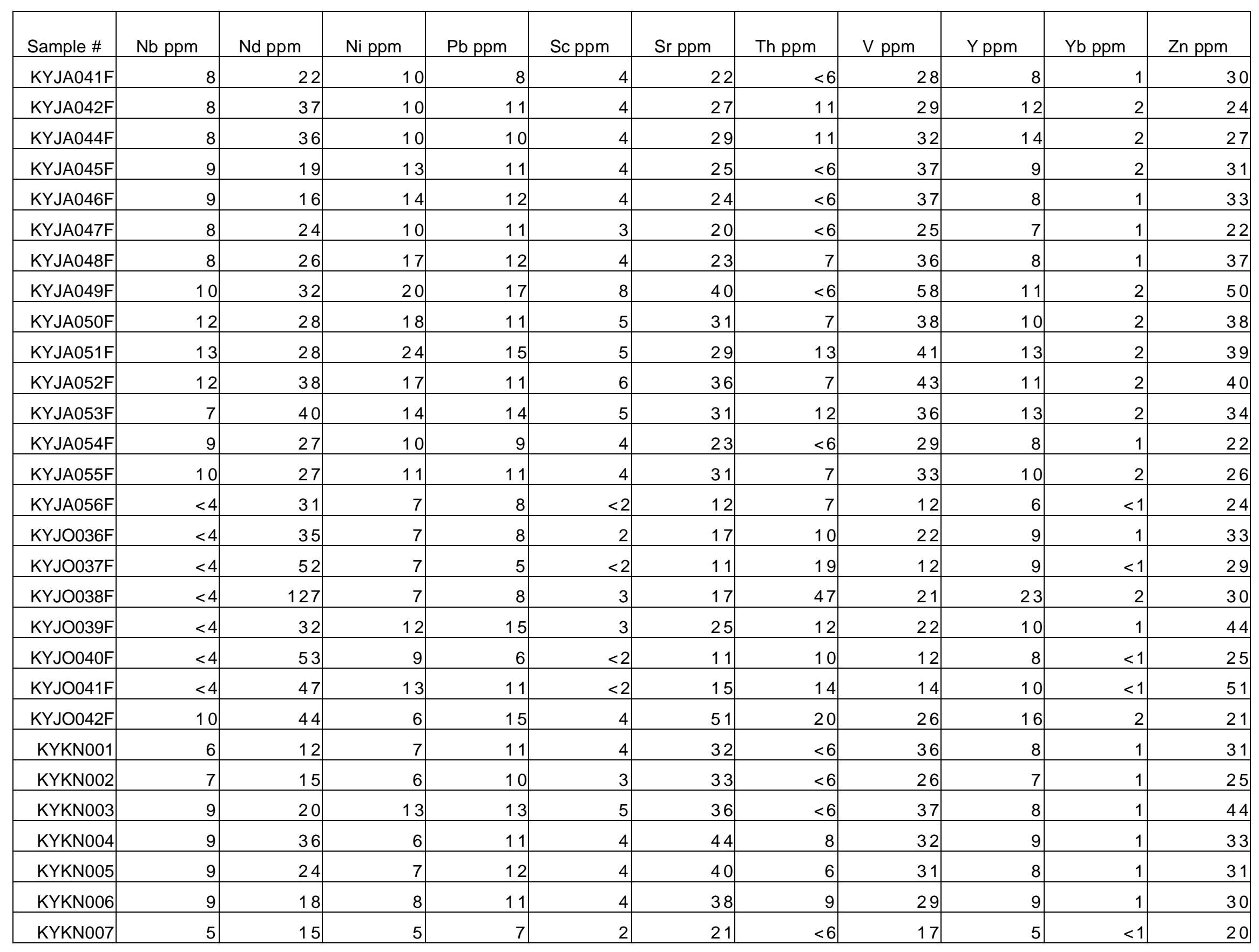




\begin{tabular}{|c|c|c|c|c|c|c|c|c|c|c|c|}
\hline Sample \# & $\mathrm{Nb}$ ppm & $\mathrm{Nd} \mathrm{ppm}$ & Ni ppm & $\mathrm{Pb}$ ppm & Sc ppm & Sr ppm & Th ppm & V ppm & Y ppm & Yb ppm & Zn ppm \\
\hline KYKN008 & 8 & 28 & 8 & 13 & 5 & 40 & 9 & 36 & 8 & 1 & 37 \\
\hline KYKN009 & 12 & 21 & 13 & 16 & 7 & 58 & $<6$ & 56 & 13 & 2 & 58 \\
\hline KYKN011 & 8 & 23 & 9 & 12 & 4 & 41 & $<6$ & 30 & 7 & 1 & 30 \\
\hline KYKN012 & 10 & 24 & 15 & 17 & 7 & 52 & 9 & 55 & 11 & 2 & 56 \\
\hline KYKN015 & 6 & 21 & 6 & 9 & 3 & 27 & $<6$ & 20 & 5 & $<1$ & 23 \\
\hline KYKN016 & 7 & 31 & 6 & 9 & 3 & 35 & 7 & 21 & 8 & 1 & 22 \\
\hline KYKN017 & 5 & 18 & 5 & 7 & $<2$ & 26 & $<6$ & 13 & 4 & $<1$ & 16 \\
\hline KYKN018 & 7 & 27 & 7 & 9 & 3 & 37 & $<6$ & 25 & 7 & 1 & 24 \\
\hline KYKN019 & 6 & 28 & 10 & 8 & 3 & 34 & $<6$ & 25 & 6 & 1 & 25 \\
\hline KYKN023 & 10 & 29 & 17 & 15 & 5 & 43 & $<6$ & 44 & 8 & 1 & 47 \\
\hline KYKN024 & 10 & 30 & 15 & 16 & 7 & 51 & 7 & 55 & 9 & 2 & 53 \\
\hline KYKN025 & 7 & 28 & 10 & 13 & 5 & 37 & $<6$ & 38 & 7 & 1 & 36 \\
\hline KYKN026 & 7 & 28 & 11 & 15 & 7 & 46 & $<6$ & 54 & 12 & 2 & 61 \\
\hline KYKN027 & 8 & 20 & 10 & 13 & 5 & 40 & $<6$ & 45 & 8 & 2 & 100 \\
\hline KYKN028 & 6 & 18 & 13 & 14 & 4 & 37 & $<6$ & 35 & 7 & 1 & 37 \\
\hline KYKN029 & 5 & 21 & 6 & 9 & 2 & 29 & $<6$ & 18 & 5 & $<1$ & 19 \\
\hline KYKN030 & 5 & 15 & 12 & 10 & 4 & 36 & $<6$ & 33 & 6 & 1 & 33 \\
\hline KYKN031 & 4 & 29 & 6 & 8 & 2 & 26 & $<6$ & 19 & 4 & $<1$ & 22 \\
\hline KYKN032 & 9 & 20 & 10 & 12 & 5 & 49 & $<6$ & 36 & 9 & 2 & 30 \\
\hline
\end{tabular}




\begin{tabular}{|c|c|c|c|c|c|c|c|c|c|c|c|}
\hline Sample \# & $\mathrm{Nb}$ ppm & $\mathrm{Nd} \mathrm{ppm}$ & Ni ppm & $\mathrm{Pb} p p m$ & Sc ppm & Sr ppm & Th ppm & V ppm & Y ppm & Yb ppm & Zn ppm \\
\hline KYKN038 & 7 & 20 & 9 & 11 & 4 & 35 & $<6$ & 30 & 6 & 1 & 32 \\
\hline KYKN039 & 7 & 20 & 10 & 12 & 4 & 38 & $<6$ & 32 & 7 & 1 & 33 \\
\hline KYKN041 & 6 & 18 & 7 & 10 & 3 & 32 & $<6$ & 23 & 5 & $<1$ & 24 \\
\hline KYKN042 & 8 & 19 & 10 & 10 & 4 & 63 & $<6$ & 33 & 7 & 1 & 38 \\
\hline KYKN045 & 6 & 12 & 7 & 8 & 3 & 35 & $<6$ & 27 & 5 & 1 & 29 \\
\hline KYKN046 & 6 & 27 & 8 & 11 & 4 & 38 & $<6$ & 30 & 6 & 1 & 37 \\
\hline KYKN047 & 8 & 22 & 9 & 11 & 4 & 42 & $<6$ & 31 & 6 & 1 & 35 \\
\hline KYKN048 & 7 & 22 & 8 & 10 & 4 & 34 & $<6$ & 30 & 5 & 1 & 29 \\
\hline KYKN049 & 6 & 22 & 8 & 10 & 3 & 45 & $<6$ & 22 & 5 & $<1$ & 27 \\
\hline KYKN054 & 5 & 23 & 3 & 7 & $<2$ & 27 & $<6$ & 17 & 4 & $<1$ & 17 \\
\hline KYKN055 & 4 & 20 & 6 & 10 & 2 & 28 & $<6$ & 17 & 4 & $<1$ & 19 \\
\hline KYKN056 & 6 & 13 & 6 & 8 & 3 & 28 & $<6$ & 25 & 6 & $<1$ & 26 \\
\hline KYKN057 & $<4$ & 11 & $<3$ & 4 & $<2$ & 17 & $<6$ & 10 & 3 & $<1$ & 12 \\
\hline KYKN058 & 4 & 18 & 5 & 7 & $<2$ & 22 & $<6$ & 14 & 4 & $<1$ & 17 \\
\hline KYKN059 & 5 & 12 & 5 & 9 & 2 & 29 & $<6$ & 21 & 4 & $<1$ & 22 \\
\hline KYKN060 & 5 & 22 & 6 & 9 & 2 & 29 & $<6$ & 20 & 5 & $<1$ & 25 \\
\hline KYKN061 & 5 & 10 & $<3$ & 7 & $<2$ & 31 & $<6$ & 15 & 4 & $<1$ & 16 \\
\hline KYKN062 & $<4$ & 14 & 3 & 6 & $<2$ & 19 & $<6$ & 12 & 3 & $<1$ & 16 \\
\hline KYKN063 & $<4$ & 12 & 3 & 5 & $<2$ & 19 & $<6$ & 12 & 3 & $<1$ & 12 \\
\hline
\end{tabular}




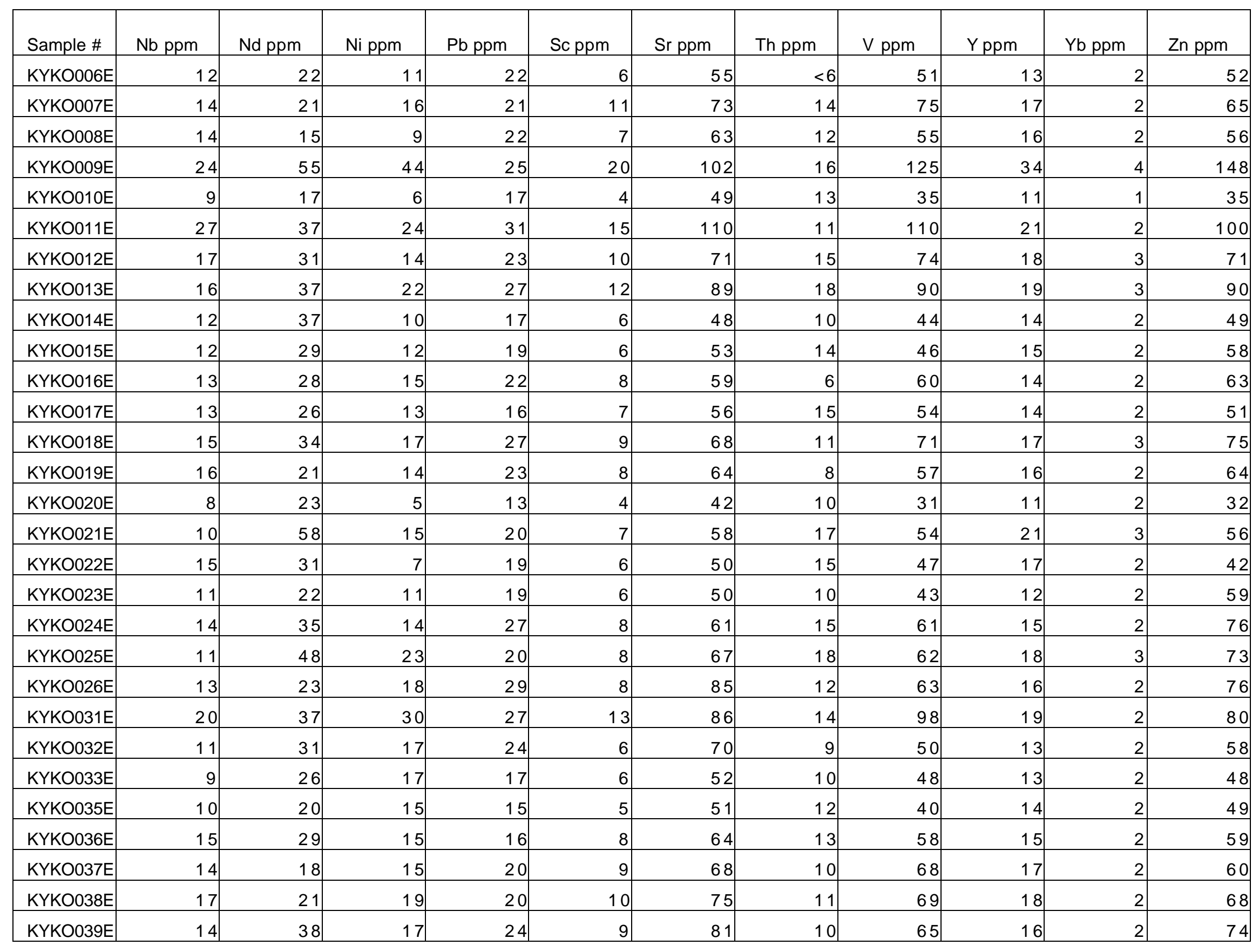




\begin{tabular}{|c|c|c|c|c|c|c|c|c|c|c|c|}
\hline KYLU001 & 6 & 21 & 15 & 8 & 3 & 19 & $<6$ & 22 & 7 & $<1$ & 28 \\
\hline KYLU003 & 9 & 19 & 9 & 12 & 4 & 39 & 11 & 30 & 9 & 1 & 21 \\
\hline KYLU005 & 14 & 49 & 34 & 29 & 10 & 62 & 9 & 67 & 18 & 2 & 74 \\
\hline KYLU008 & 10 & 22 & 17 & 17 & 6 & 33 & 8 & 47 & 11 & 2 & 42 \\
\hline KYLU009 & 15 & 37 & 17 & 19 & 8 & 49 & 15 & 63 & 16 & 2 & 43 \\
\hline KYLU010 & 14 & 53 & 15 & 15 & 7 & 53 & 13 & 51 & 13 & 2 & 51 \\
\hline KYLU011 & 16 & 39 & 23 & 26 & 10 & 74 & 12 & 73 & 15 & 2 & 74 \\
\hline KYLU012 & 11 & 38 & 12 & 19 & 6 & 54 & 17 & 43 & 13 & 2 & 40 \\
\hline KYLU016 & 12 & 30 & 16 & 13 & 7 & 40 & 12 & 52 & 13 & 2 & 40 \\
\hline KYLU017 & 16 & 31 & 22 & 24 & 8 & 50 & 14 & 66 & 14 & 2 & 53 \\
\hline KYLU018 & 18 & 37 & 24 & 22 & 14 & 67 & 15 & 101 & 17 & 3 & 74 \\
\hline KYLU019 & 15 & 23 & 24 & 15 & 6 & 34 & 8 & 46 & 12 & 2 & 43 \\
\hline KYLU020 & 13 & 17 & 16 & 19 & 7 & 60 & 9 & 54 & 11 & 2 & 36 \\
\hline KYLU021 & 15 & 27 & 16 & 47 & 6 & 40 & 10 & 47 & 11 & 2 & 43 \\
\hline KYLU022 & 11 & 34 & 33 & 23 & 8 & 47 & 11 & 63 & 14 & 2 & 64 \\
\hline KYLU023 & 11 & 15 & 12 & 16 & 5 & 29 & 10 & 39 & 9 & 1 & 35 \\
\hline KYLU024 & 13 & 25 & 15 & 16 & 6 & 41 & 12 & 50 & 11 & 2 & 41 \\
\hline KYLU025 & 7 & 19 & 9 & 9 & 3 & 19 & 7 & 20 & 7 & 1 & 21 \\
\hline
\end{tabular}




\begin{tabular}{|c|c|c|c|c|c|c|c|c|c|c|c|}
\hline Sample \# & $\mathrm{Nb}$ ppm & Nd ppm & Ni ppm & $\mathrm{Pb} \mathrm{ppm}$ & Sc ppm & Sr ppm & Th ppm & $\mathrm{V}$ ppm & Y ppm & Yb ppm & Zn ppm \\
\hline KYLU031 & 9 & 31 & 18 & 35 & 6 & 49 & 9 & 43 & 10 & 1 & 57 \\
\hline KYLU033 & 13 & 37 & 16 & 23 & 7 & 69 & 11 & 56 & 14 & 2 & 44 \\
\hline KYLU034 & 16 & 32 & 14 & 15 & 8 & 49 & 13 & 61 & 13 & 2 & 43 \\
\hline KYLU037 & 16 & 34 & 26 & 17 & 9 & 52 & 12 & 67 & 16 & 2 & 57 \\
\hline KYLU038 & 11 & 38 & 26 & 23 & 8 & 51 & 16 & 61 & 14 & 2 & 57 \\
\hline KYLU039 & 18 & 49 & 26 & 25 & 9 & 56 & 14 & 71 & 16 & 2 & 60 \\
\hline KYLU040 & 18 & 34 & 44 & 24 & 14 & 69 & 19 & 110 & 19 & 3 & 79 \\
\hline KYLW004F & 19 & 39 & 20 & 15 & 11 & 59 & 15 & 77 & 17 & 3 & 56 \\
\hline KYLE002 & 12 & 31 & 32 & 29 & 12 & 62 & 12 & 99 & 15 & 2 & 85 \\
\hline KYLE003 & 17 & 39 & 31 & 32 & 12 & 67 & 15 & 96 & 15 & 2 & 96 \\
\hline KYLE004 & 10 & 25 & 18 & 16 & 7 & 45 & 10 & 57 & 10 & 2 & 60 \\
\hline KYLE005 & 9 & 32 & 21 & 20 & 7 & 43 & 7 & 61 & 10 & 1 & 61 \\
\hline KYLE006 & 8 & 32 & 28 & 32 & 8 & 56 & 10 & 69 & 12 & 2 & 65 \\
\hline KYLE007 & 8 & 37 & 16 & 18 & 6 & 53 & $<6$ & 51 & 9 & 2 & 51 \\
\hline KYLE008 & 8 & 17 & 15 & 17 & 5 & 42 & $<6$ & 45 & 8 & 2 & 53 \\
\hline KYLE009 & 7 & 21 & 17 & 277 & 6 & 50 & $<6$ & 48 & 10 & 2 & 65 \\
\hline KYLE010 & $<4$ & 20 & 9 & 9 & 2 & 25 & $<6$ & 23 & 5 & $<1$ & 24 \\
\hline KYLE011 & 6 & 25 & 16 & 10 & 4 & 22 & $<6$ & 33 & 7 & 1 & 30 \\
\hline
\end{tabular}




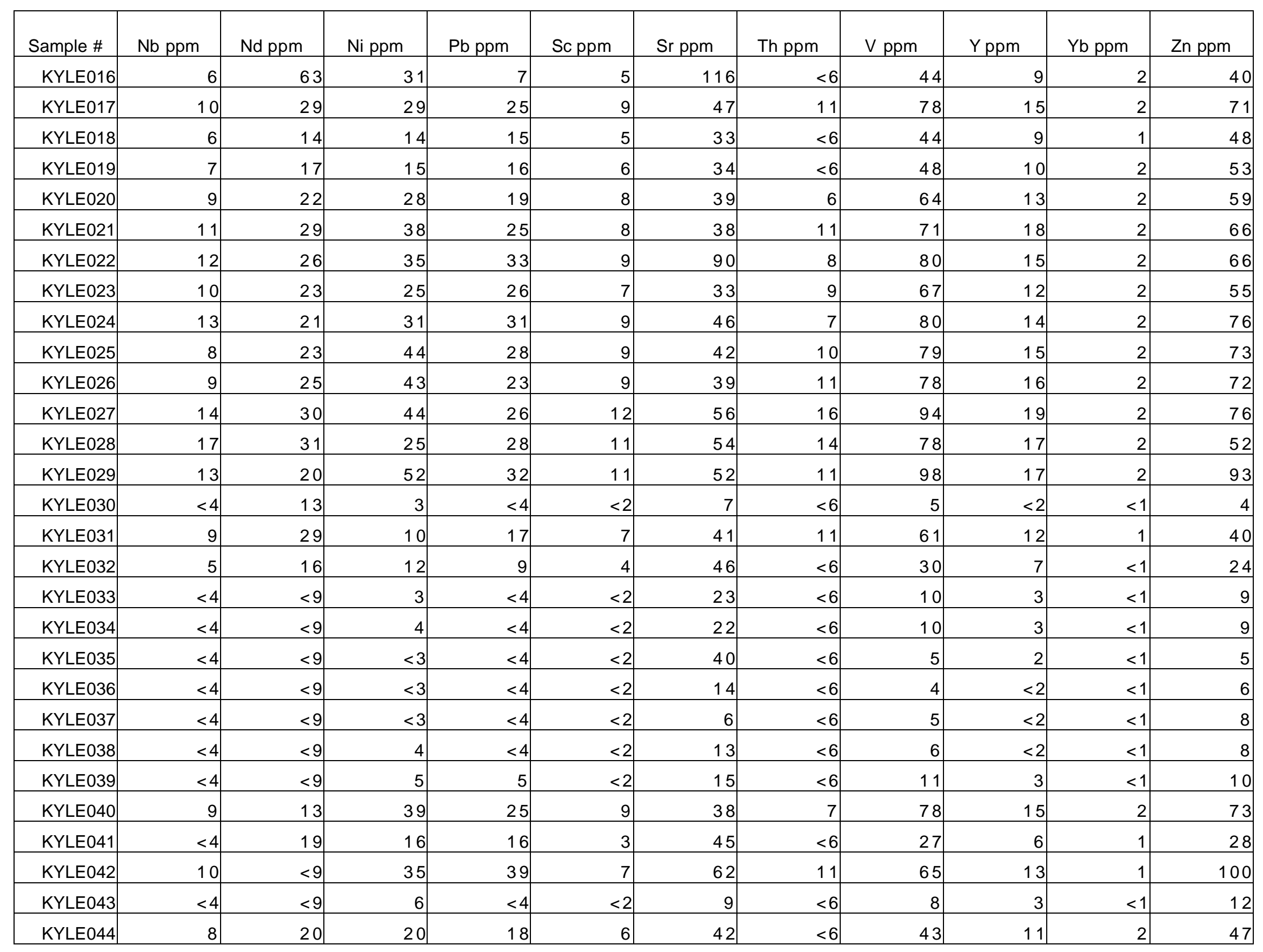




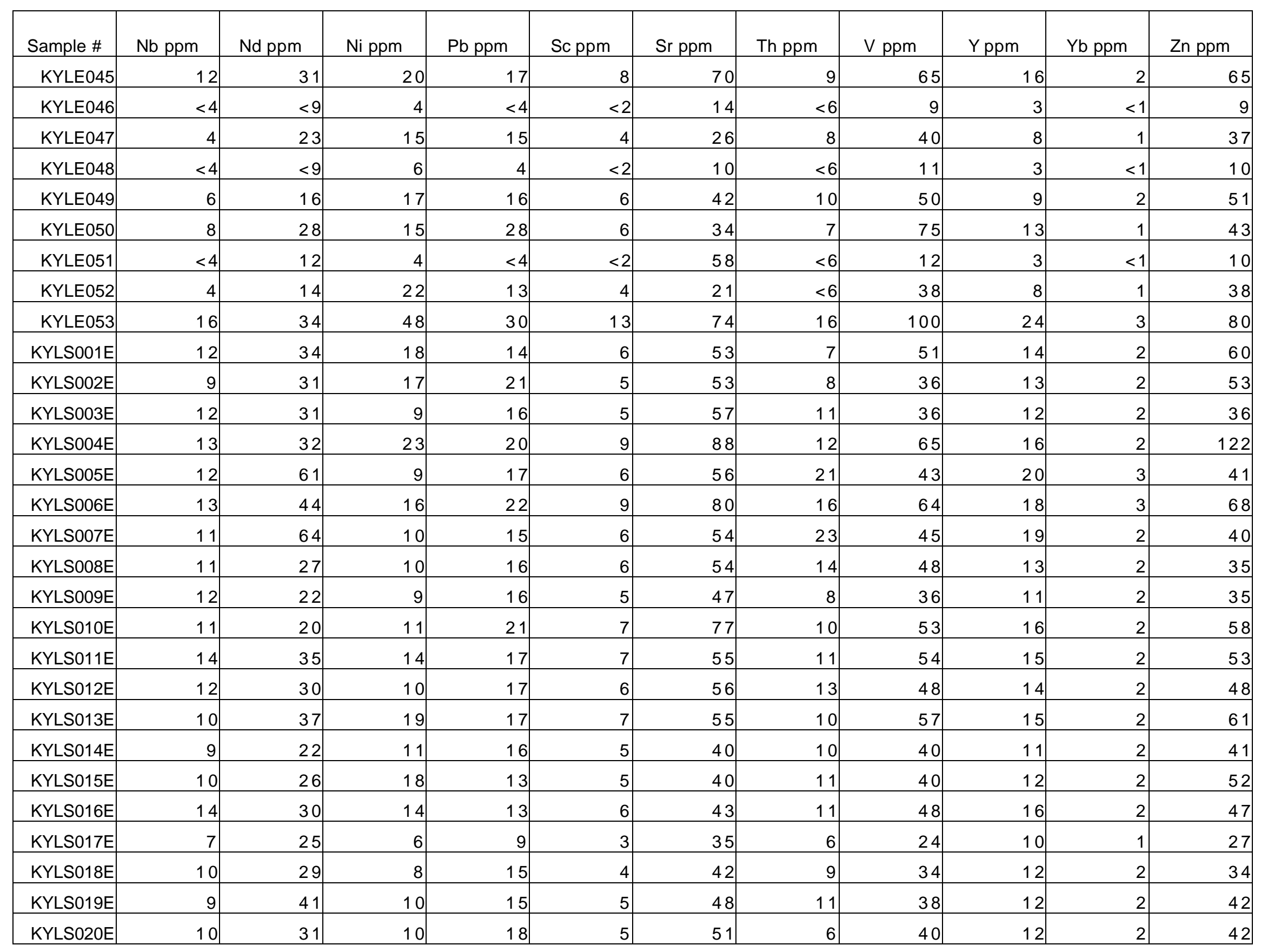




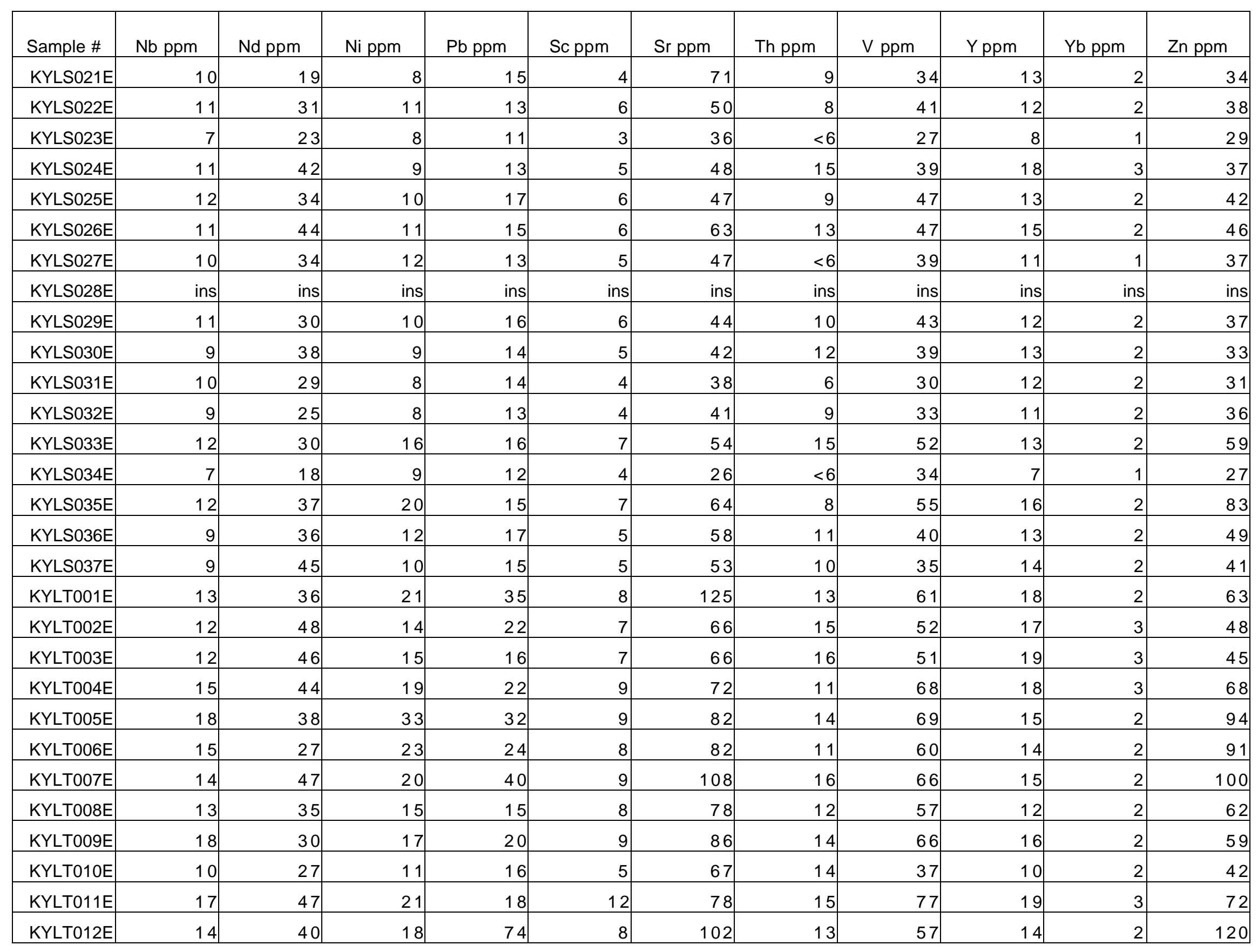




\begin{tabular}{|c|c|c|c|c|c|c|c|c|c|c|c|}
\hline Sample \# & $\mathrm{Nb}$ ppm & $\mathrm{Nd} \mathrm{ppm}$ & Ni ppm & $\mathrm{Pb}$ ppm & Sc ppm & Sr ppm & Th ppm & V ppm & Y ppm & Yb ppm & Zn ppm \\
\hline KYLT013E & 14 & 37 & 16 & 21 & 7 & 77 & 11 & 51 & 14 & 2 & 54 \\
\hline KYLT014E & 15 & 32 & 13 & 19 & 7 & 104 & 13 & 56 & 14 & 2 & 52 \\
\hline KYLT016E & 16 & 33 & 23 & 26 & 12 & 107 & 17 & 87 & 18 & 3 & 93 \\
\hline KYLT017E & 17 & 51 & 25 & 26 & 9 & 85 & 20 & 69 & 21 & 3 & 92 \\
\hline KYLT020E & 14 & 45 & 16 & 16 & 8 & 90 & 14 & 57 & 15 & 2 & 55 \\
\hline KYLT021E & 17 & 34 & 16 & 19 & 7 & 77 & 12 & 56 & 14 & 2 & 57 \\
\hline KYLT022E & 11 & 40 & 16 & 16 & 6 & 160 & 14 & 43 & 13 & 2 & 47 \\
\hline KYLT023E & 16 & 39 & 20 & 18 & 10 & 88 & 13 & 70 & 18 & 2 & 65 \\
\hline KYLT024E & 16 & 38 & 23 & 30 & 12 & 107 & 18 & 86 & 18 & 3 & 98 \\
\hline KYLT028E & 13 & 46 & 20 & 20 & 10 & 84 & 20 & 72 & 17 & 2 & 63 \\
\hline KYLT029E & 11 & 39 & 17 & 16 & 8 & 72 & 12 & 57 & 14 & 2 & 62 \\
\hline KYLT030E & 12 & 40 & 15 & 14 & 7 & 64 & 12 & 50 & 11 & 2 & 48 \\
\hline KYLT031E & 13 & 27 & 16 & 16 & 6 & 64 & 16 & 44 & 13 & 2 & 43 \\
\hline KYLT032E & 16 & 39 & 17 & 17 & 9 & 72 & 16 & 67 & 15 & 2 & 58 \\
\hline KYLT033E & 10 & 39 & 10 & 14 & 5 & 42 & 10 & 39 & 11 & 2 & 33 \\
\hline KYLT034E & 12 & 35 & 15 & 17 & 7 & 62 & 11 & 51 & 14 & 2 & 48 \\
\hline KYLT035E & 11 & 40 & 24 & 21 & 9 & 65 & 14 & 68 & 15 & 2 & 71 \\
\hline KYLT036E & 11 & 32 & 18 & 15 & 7 & 71 & 11 & 50 & 12 & 2 & 58 \\
\hline KYLT037E & 16 & 60 & 18 & 19 & 8 & 79 & 16 & 62 & 18 & 3 & 106 \\
\hline
\end{tabular}




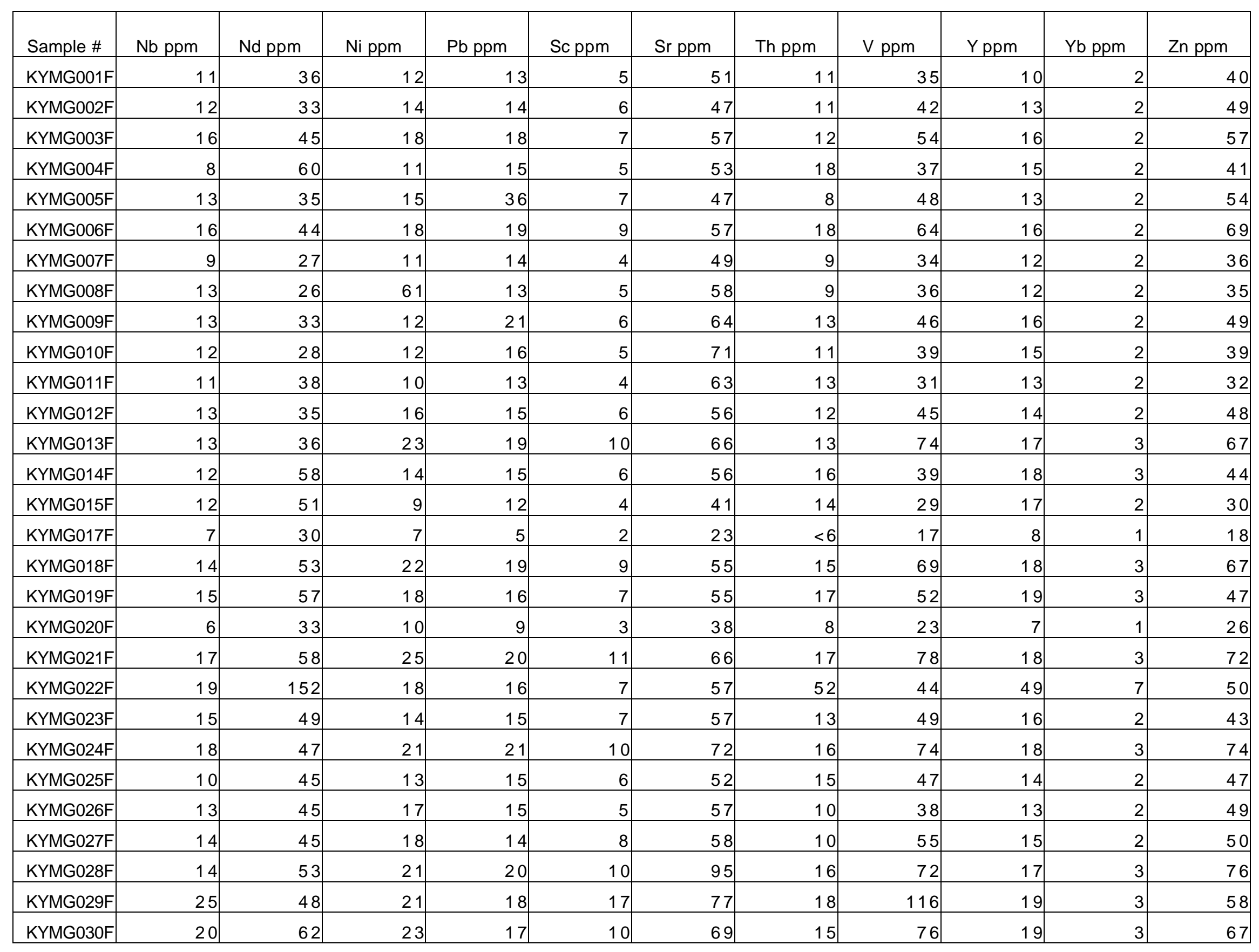




\begin{tabular}{|c|c|c|c|c|c|c|c|c|c|c|c|}
\hline Sample \# & $\mathrm{Nb}$ ppm & $\mathrm{Nd} \mathrm{ppm}$ & Ni ppm & $\mathrm{Pb}$ ppm & Sc ppm & Sr ppm & Th ppm & V ppm & Y ppm & Yb ppm & Zn ppm \\
\hline KYMG032F & 11 & 68 & 14 & 15 & 7 & 64 & 19 & 54 & 23 & 3 & 61 \\
\hline KYMG034F & 12 & 51 & 17 & 16 & 6 & 58 & 13 & 42 & 14 & 2 & 39 \\
\hline KYMG035F & 15 & 44 & 16 & 19 & 7 & 61 & 14 & 51 & 14 & 2 & 47 \\
\hline KYMG038F & 8 & 50 & 8 & 9 & 3 & 31 & 13 & 24 & 13 & 2 & 25 \\
\hline KYMG039F & 12 & 44 & 11 & 11 & 5 & 50 & 14 & 37 & 13 & 2 & 40 \\
\hline KYMG040F & 12 & 50 & 12 & 15 & 5 & 57 & 17 & 40 & 15 & 2 & 40 \\
\hline KYMG041F & 19 & 40 & 18 & 20 & 9 & 70 & 22 & 68 & 19 & 3 & 68 \\
\hline KYMG042F & 11 & 46 & 17 & 14 & 6 & 58 & 13 & 46 & 14 & 2 & 51 \\
\hline KYMG046F & 15 & 51 & 13 & 18 & 7 & 69 & 19 & 53 & 17 & 2 & 44 \\
\hline KYMG047F & 16 & 37 & 19 & 19 & 9 & 68 & 20 & 65 & 19 & 3 & 75 \\
\hline KYMG048F & 12 & 86 & 6 & 15 & 4 & 55 & 31 & 29 & 25 & 3 & 43 \\
\hline KYMG049F & 13 & 53 & 10 & 14 & 6 & 56 & 20 & 40 & 19 & 2 & 48 \\
\hline KYMG050F & 12 & 63 & 13 & 27 & 7 & 59 & 16 & 50 & 18 & 3 & 69 \\
\hline KYMG051F & 15 & 61 & 22 & 15 & 6 & 51 & 15 & 45 & 16 & 2 & 68 \\
\hline KYMG052F & 13 & 34 & 5 & 19 & 4 & 48 & 17 & 31 & 15 & 2 & 56 \\
\hline KYMG053F & 18 & 43 & 12 & 17 & 8 & 62 & 16 & 53 & 20 & 3 & 54 \\
\hline KYMG054F & 16 & 38 & 12 & 19 & 8 & 66 & 15 & 57 & 19 & 3 & 41 \\
\hline KYMG055F & 16 & 82 & 8 & 14 & 6 & 50 & 26 & 36 & 29 & 4 & 32 \\
\hline
\end{tabular}




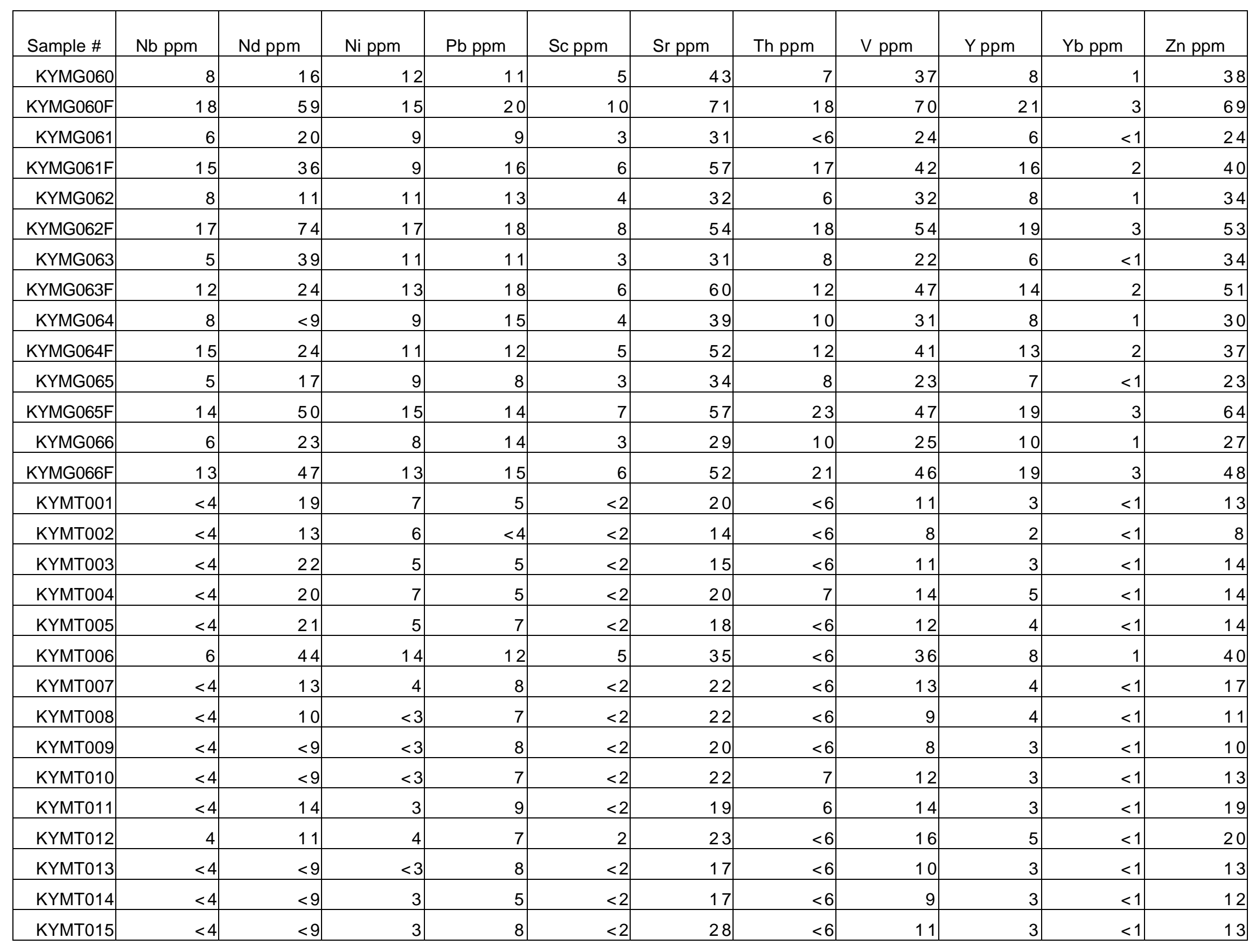




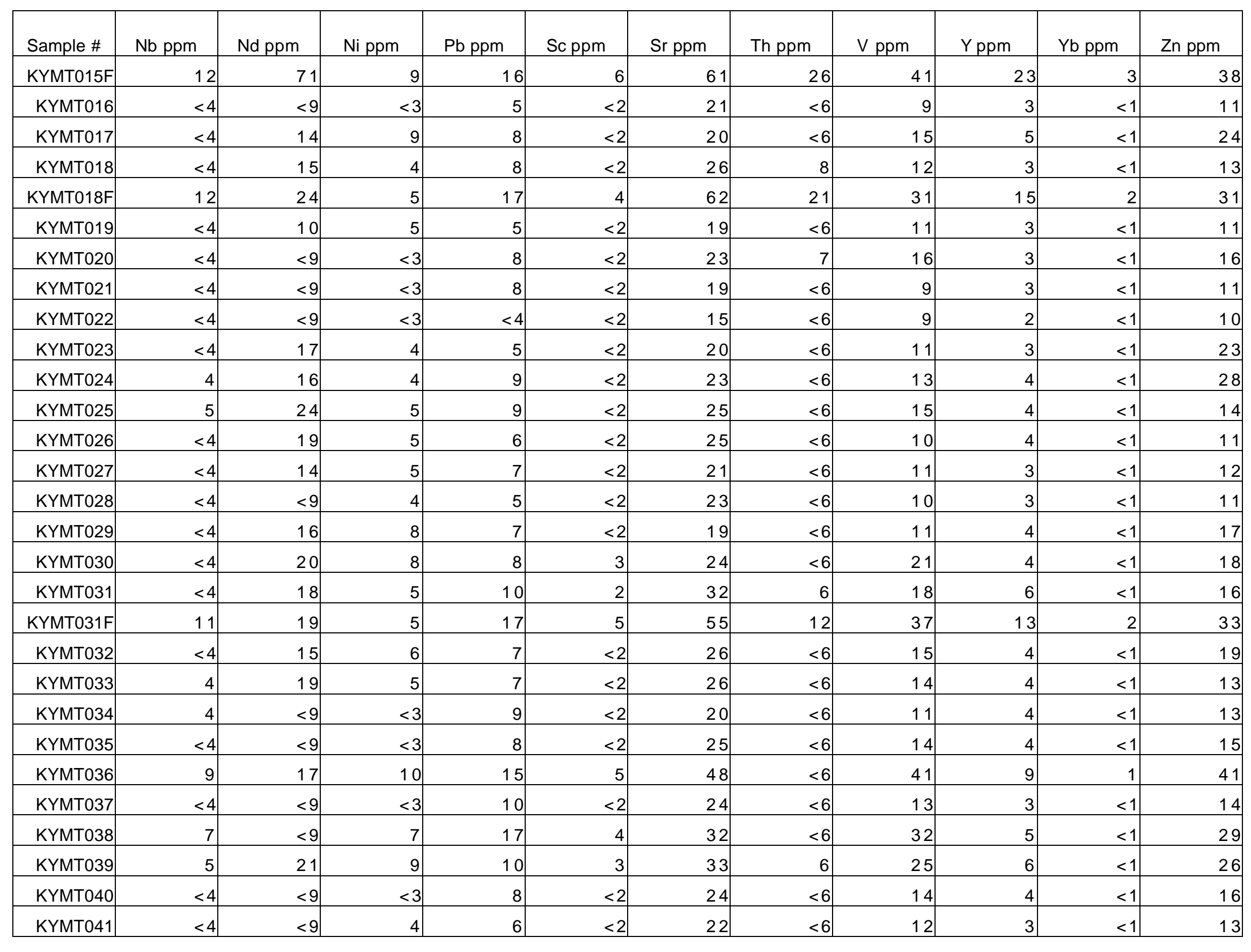




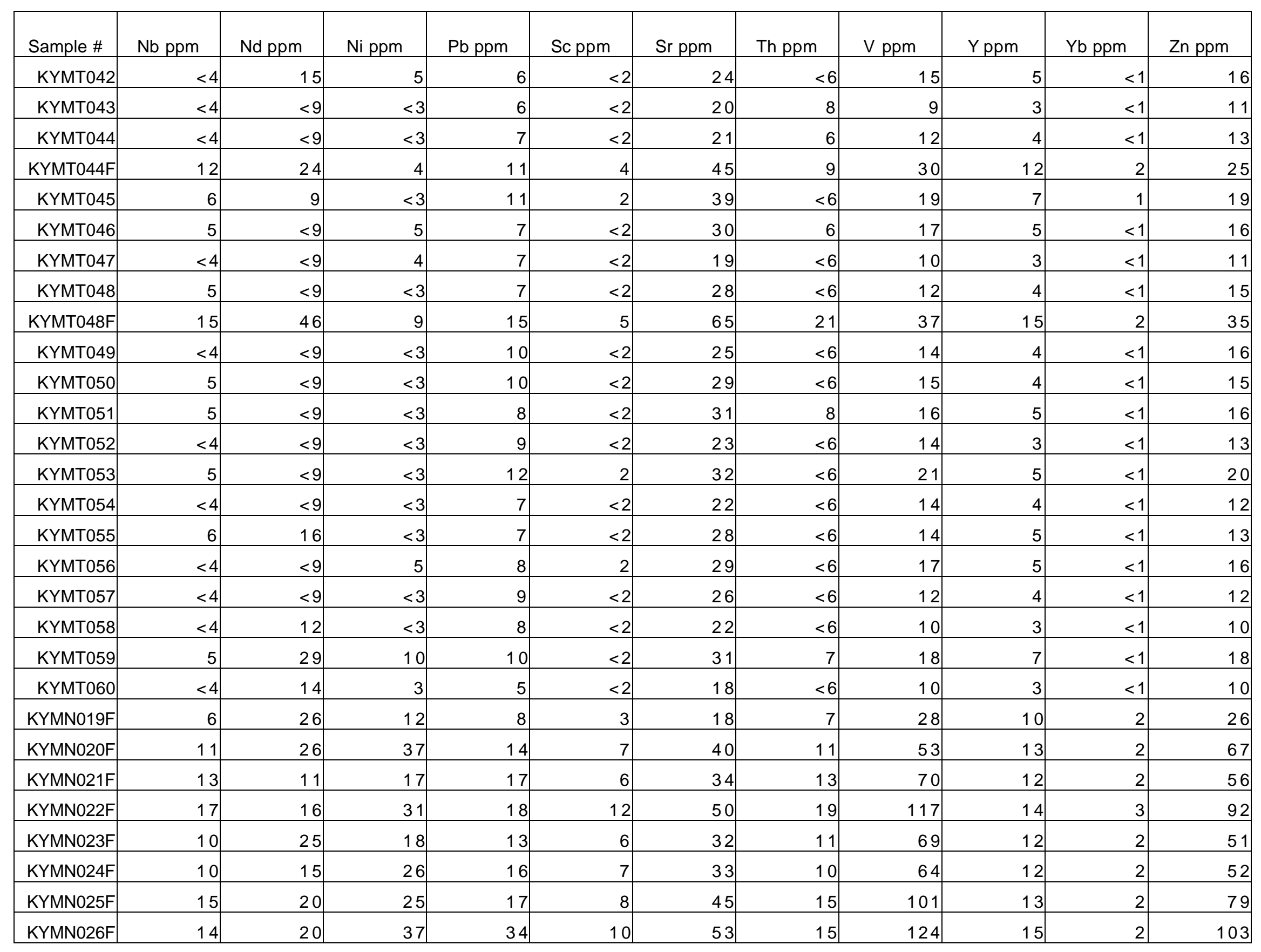




\begin{tabular}{|c|c|c|c|c|c|c|c|c|c|c|c|}
\hline KYMN028F & 8 & 39 & 25 & 12 & 5 & 47 & 9 & 50 & 11 & 2 & 51 \\
\hline KYOR035F & 9 & 32 & 10 & 12 & 4 & 41 & 13 & 31 & 16 & 2 & 24 \\
\hline KYOR037 & $<4$ & $<9$ & 7 & 4 & $<2$ & 11 & $<6$ & 13 & 4 & $<1$ & 13 \\
\hline KYOR039F & 13 & 31 & 17 & 15 & 6 & 47 & 14 & 47 & 13 & 2 & 37 \\
\hline KYOR040 & 6 & 11 & 9 & 11 & 3 & 22 & 8 & 23 & 6 & 1 & 20 \\
\hline KYOR041 & 7 & $<9$ & 10 & 12 & 4 & 32 & $<6$ & 31 & 7 & 1 & 31 \\
\hline KYOR041F & 11 & 17 & 14 & 15 & 6 & 49 & 12 & 43 & 15 & 2 & 38 \\
\hline KYOR042 & 6 & 11 & 10 & 11 & 3 & 30 & 6 & 28 & 7 & 1 & 26 \\
\hline KYOR045 & $<4$ & 11 & 5 & 6 & $<2$ & 25 & $<6$ & 12 & 5 & $<1$ & 13 \\
\hline KYOR046 & 6 & 16 & 8 & 8 & 3 & 35 & 7 & 23 & 6 & $<1$ & 24 \\
\hline KYOR047 & 8 & $<9$ & 16 & 14 & 5 & 32 & 8 & 40 & 10 & 1 & 42 \\
\hline KYOR048 & 19 & 27 & 24 & 25 & 11 & 61 & 15 & 82 & 19 & 3 & 72 \\
\hline KYOR048F & 18 & 41 & 26 & 23 & 12 & 68 & 16 & 83 & 20 & 3 & 73 \\
\hline KYOR049 & 9 & 17 & 20 & 18 & 7 & 42 & 10 & 60 & 12 & 2 & 58 \\
\hline KYOR050 & 7 & 12 & 12 & 15 & 4 & 29 & 8 & 37 & 8 & 1 & 39 \\
\hline KYOR051 & 7 & $<9$ & 11 & 11 & 4 & 30 & $<6$ & 33 & 7 & 1 & 31 \\
\hline KYOS001 & 11 & 39 & 29 & 26 & 9 & 62 & 11 & 78 & 13 & 2 & 105 \\
\hline KYOS002 & 7 & $<9$ & 13 & 15 & 4 & 97 & 7 & 37 & 9 & 1 & 40 \\
\hline
\end{tabular}




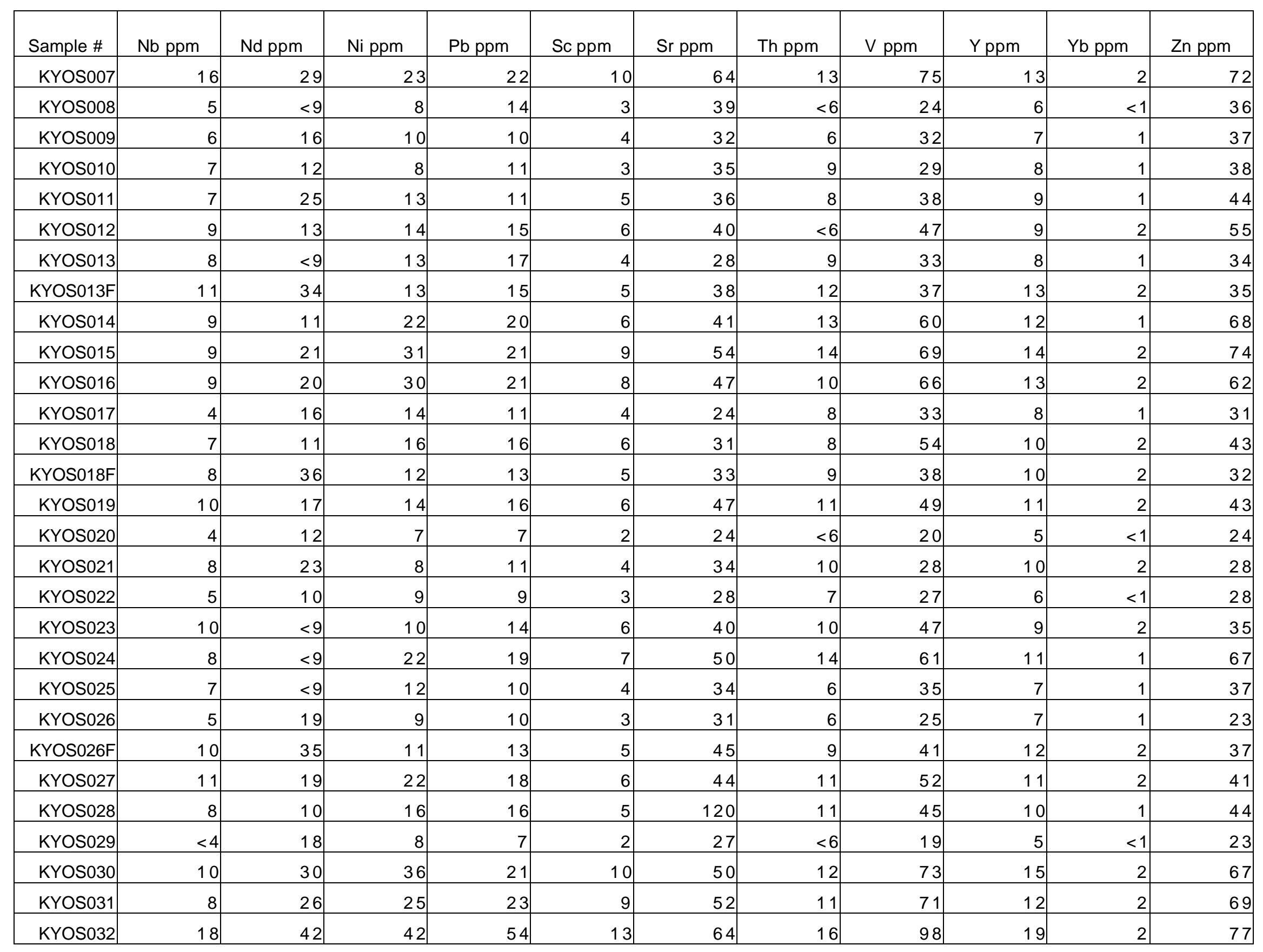




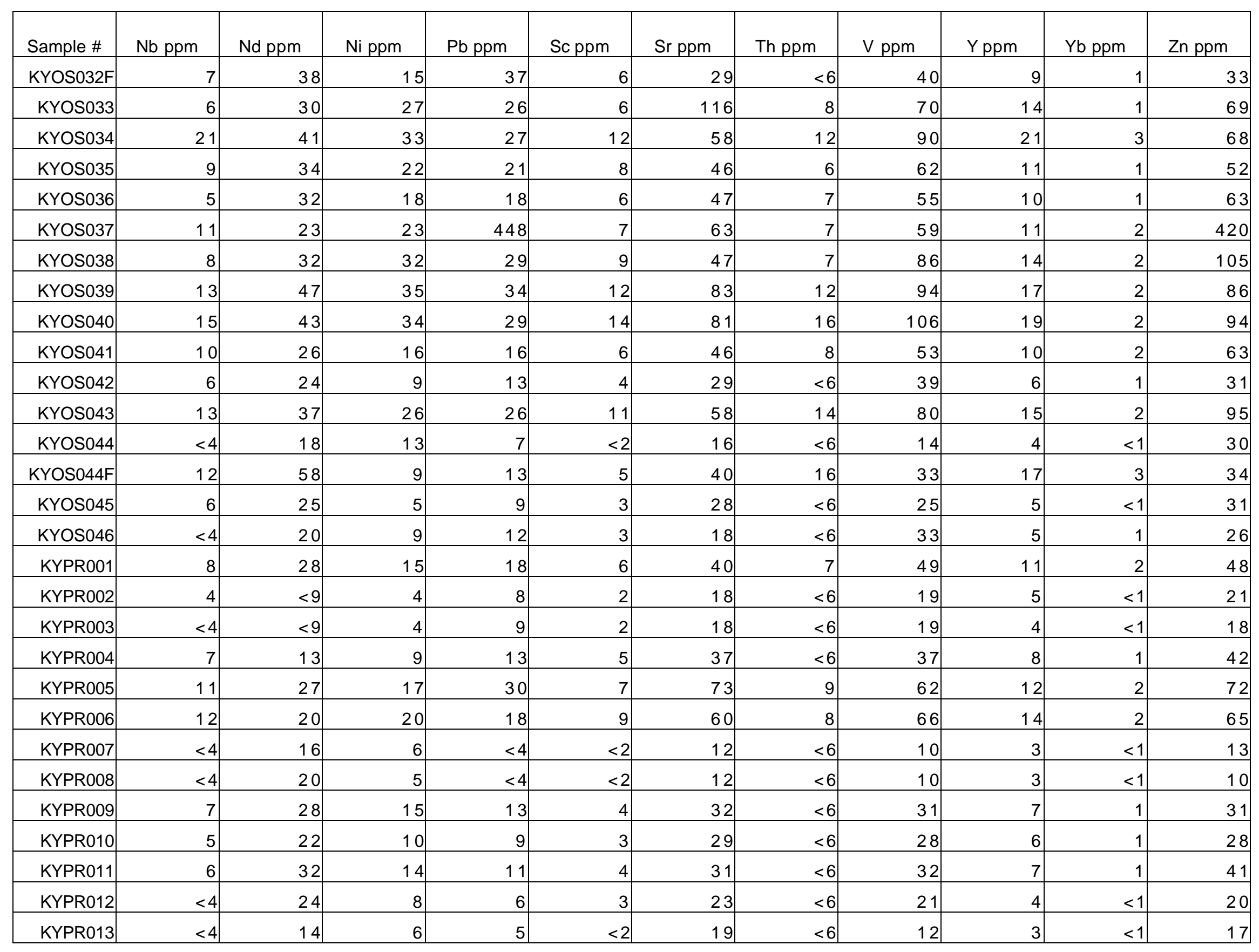




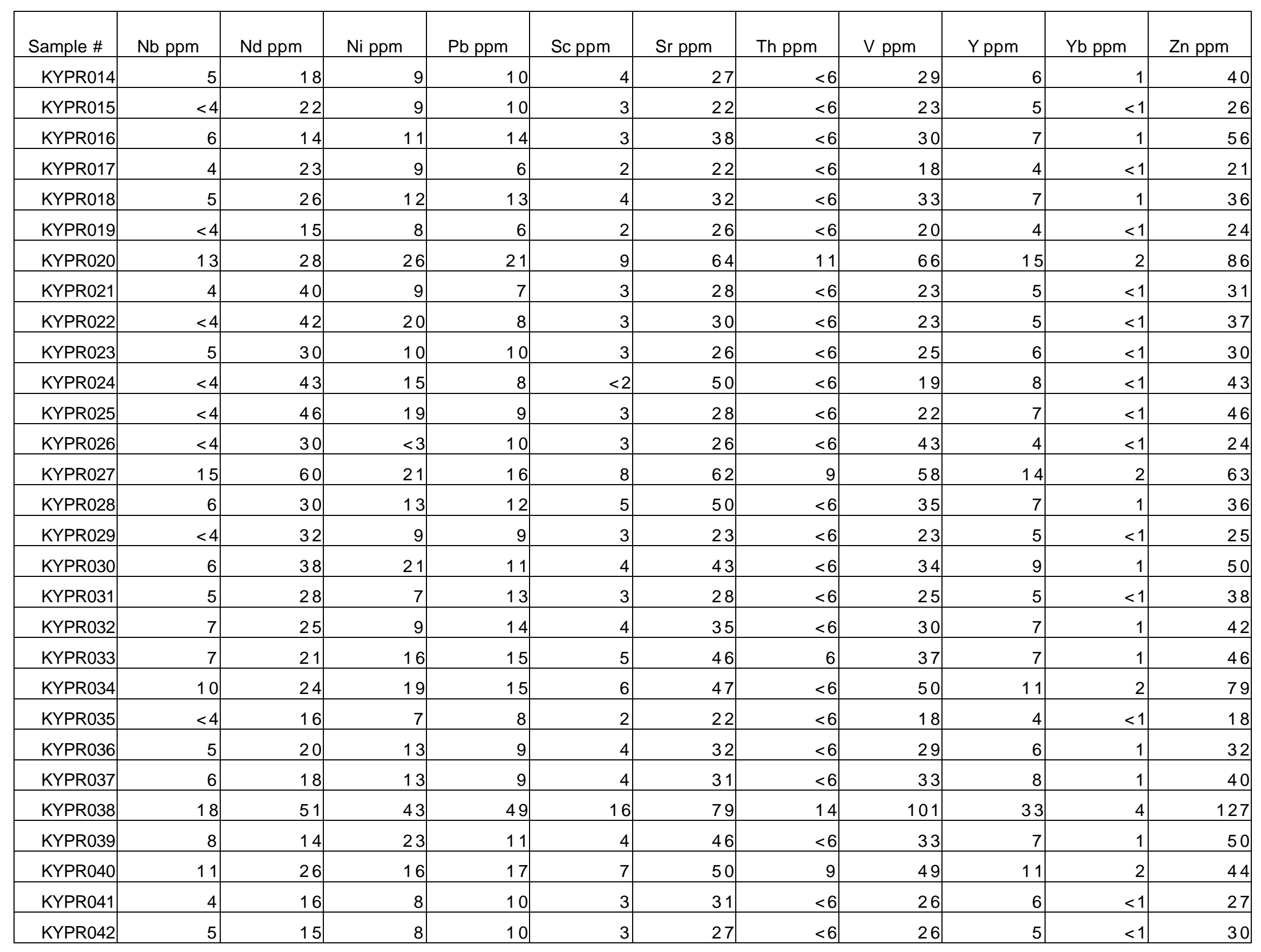




\begin{tabular}{|c|c|c|c|c|c|c|c|c|c|c|c|}
\hline KYPR044 & 5 & 18 & 9 & 9 & 3 & 27 & $<6$ & 30 & 5 & $<1$ & 29 \\
\hline KYPR046 & 16 & 29 & 19 & 19 & 9 & 61 & 12 & 62 & 13 & 2 & 56 \\
\hline KYPR047 & 5 & 17 & 8 & 8 & 3 & 25 & $<6$ & 27 & 6 & 1 & 24 \\
\hline KYPR050 & $<4$ & 21 & 8 & 7 & $<2$ & 21 & $<6$ & 14 & 4 & $<1$ & 28 \\
\hline KYPR051 & 10 & 24 & 18 & 16 & 8 & 60 & 8 & 62 & 12 & 2 & 51 \\
\hline KYPR052 & 5 & 21 & 9 & 10 & 4 & 34 & $<6$ & 27 & 7 & 1 & 26 \\
\hline KYPR053 & $<4$ & 12 & 6 & 9 & $<2$ & 19 & $<6$ & 14 & 3 & $<1$ & 17 \\
\hline KYPR054 & 4 & 19 & 17 & 12 & 4 & 38 & $<6$ & 36 & 7 & 1 & 55 \\
\hline KYPR060 & 9 & 26 & 16 & 17 & 7 & 48 & 9 & 55 & 9 & 2 & 49 \\
\hline KYPR061 & 7 & 21 & 18 & 13 & 5 & 40 & $<6$ & 41 & 8 & 1 & 46 \\
\hline KYPR062 & 6 & 19 & 11 & 12 & 4 & 31 & $<6$ & 30 & 6 & 1 & 26 \\
\hline KYPR063 & 5 & 15 & 9 & 11 & 4 & 26 & $<6$ & 30 & 5 & $<1$ & 27 \\
\hline KYPR064 & 4 & 23 & 8 & 11 & 4 & 35 & $<6$ & 34 & 5 & 1 & 25 \\
\hline KYPR065 & 5 & 32 & 15 & 9 & 4 & 32 & 7 & 28 & 7 & 1 & 33 \\
\hline KYPR066 & 7 & 26 & 12 & 11 & 5 & 34 & 9 & 36 & 8 & 1 & 33 \\
\hline KYPR067 & 6 & 29 & 10 & 8 & 3 & 30 & $<6$ & 26 & 6 & $<1$ & 27 \\
\hline KYPR068 & $<4$ & 20 & 8 & 7 & $<2$ & 18 & 6 & 15 & 4 & $<1$ & 17 \\
\hline KYPR069 & 6 & 20 & 8 & 5 & 2 & 22 & $<6$ & 18 & 4 & $<1$ & 16 \\
\hline
\end{tabular}




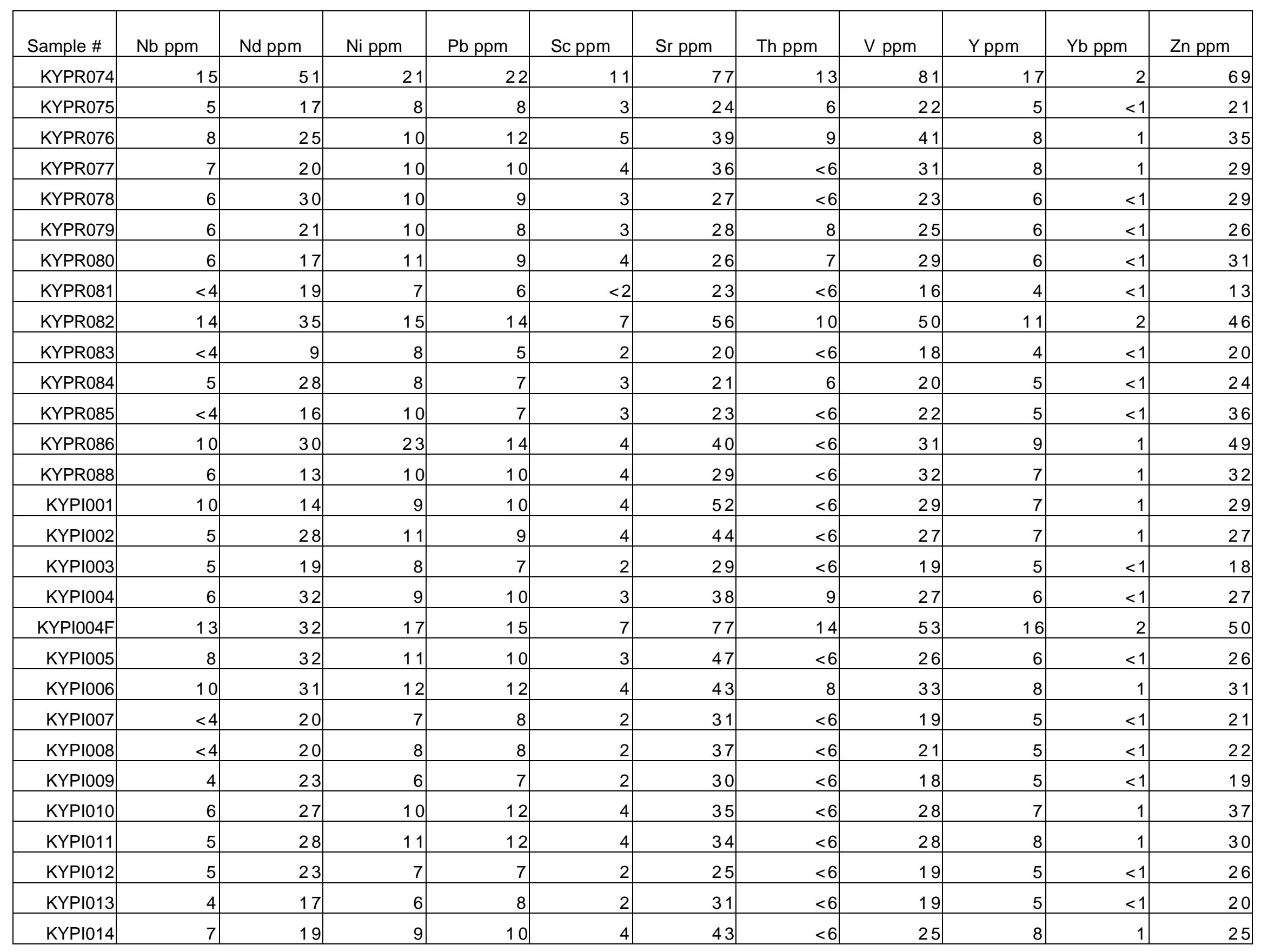




\begin{tabular}{|c|c|c|c|c|c|c|c|c|c|c|c|}
\hline Sample \# & $\mathrm{Nb}$ ppm & $\mathrm{Nd} \mathrm{ppm}$ & Ni ppm & $\mathrm{Pb}$ ppm & Sc ppm & Sr ppm & Th ppm & V ppm & Y ppm & Yb ppm & Zn ppm \\
\hline KYPI015 & 4 & 17 & 6 & 8 & 3 & 35 & $<6$ & 19 & 6 & $<1$ & 20 \\
\hline KYPI016 & 5 & 20 & 9 & 9 & 3 & 39 & $<6$ & 26 & 6 & $<1$ & 29 \\
\hline KYPI018 & 6 & 38 & 17 & 27 & 4 & 71 & 8 & 33 & 9 & 1 & 41 \\
\hline KYPI018F & 14 & 42 & 25 & 67 & 7 & 102 & 11 & 53 & 17 & 2 & 66 \\
\hline KYPI020 & 7 & 17 & 10 & 12 & 4 & 36 & $<6$ & 33 & 7 & 1 & 34 \\
\hline KYPI021 & 7 & 31 & 10 & 9 & 4 & 45 & 8 & 33 & 8 & 1 & 30 \\
\hline KYPI022 & 4 & 9 & 5 & 9 & 3 & 32 & $<6$ & 21 & 5 & $<1$ & 24 \\
\hline KYPI023 & 6 & 20 & 9 & 9 & 3 & 43 & 6 & 25 & 6 & $<1$ & 24 \\
\hline KYPI024 & 6 & 30 & 11 & 11 & 4 & 40 & 8 & 30 & 6 & 1 & 31 \\
\hline KYPI067 & 5 & $<9$ & 8 & 9 & 3 & 32 & $<6$ & 21 & 5 & $<1$ & 40 \\
\hline KYPI068 & 6 & 17 & 10 & 9 & 3 & 45 & $<6$ & 27 & 6 & $<1$ & 28 \\
\hline KYPI069 & 10 & 17 & 14 & 17 & 7 & 59 & 11 & 52 & 11 & 2 & 53 \\
\hline KYPI070 & 6 & $<9$ & 9 & 12 & 4 & 56 & $<6$ & 31 & 7 & 1 & 40 \\
\hline KYPI071 & 6 & 12 & 9 & 30 & 3 & 64 & $<6$ & 28 & 7 & $<1$ & 38 \\
\hline KYPI072 & 7 & $<9$ & 7 & 13 & 3 & 49 & $<6$ & 26 & 6 & $<1$ & 26 \\
\hline KYPI073 & 6 & $<9$ & 8 & 15 & 3 & 47 & $<6$ & 26 & 6 & $<1$ & 28 \\
\hline KYPI074 & 6 & $<9$ & 7 & 10 & 3 & 47 & 6 & 26 & 6 & $<1$ & 33 \\
\hline KYPI075 & 7 & 10 & 11 & 12 & 4 & 45 & 8 & 33 & 7 & 1 & 35 \\
\hline KYPI076 & 7 & 16 & 11 & 10 & 4 & 46 & $<6$ & 32 & 7 & 1 & 61 \\
\hline
\end{tabular}




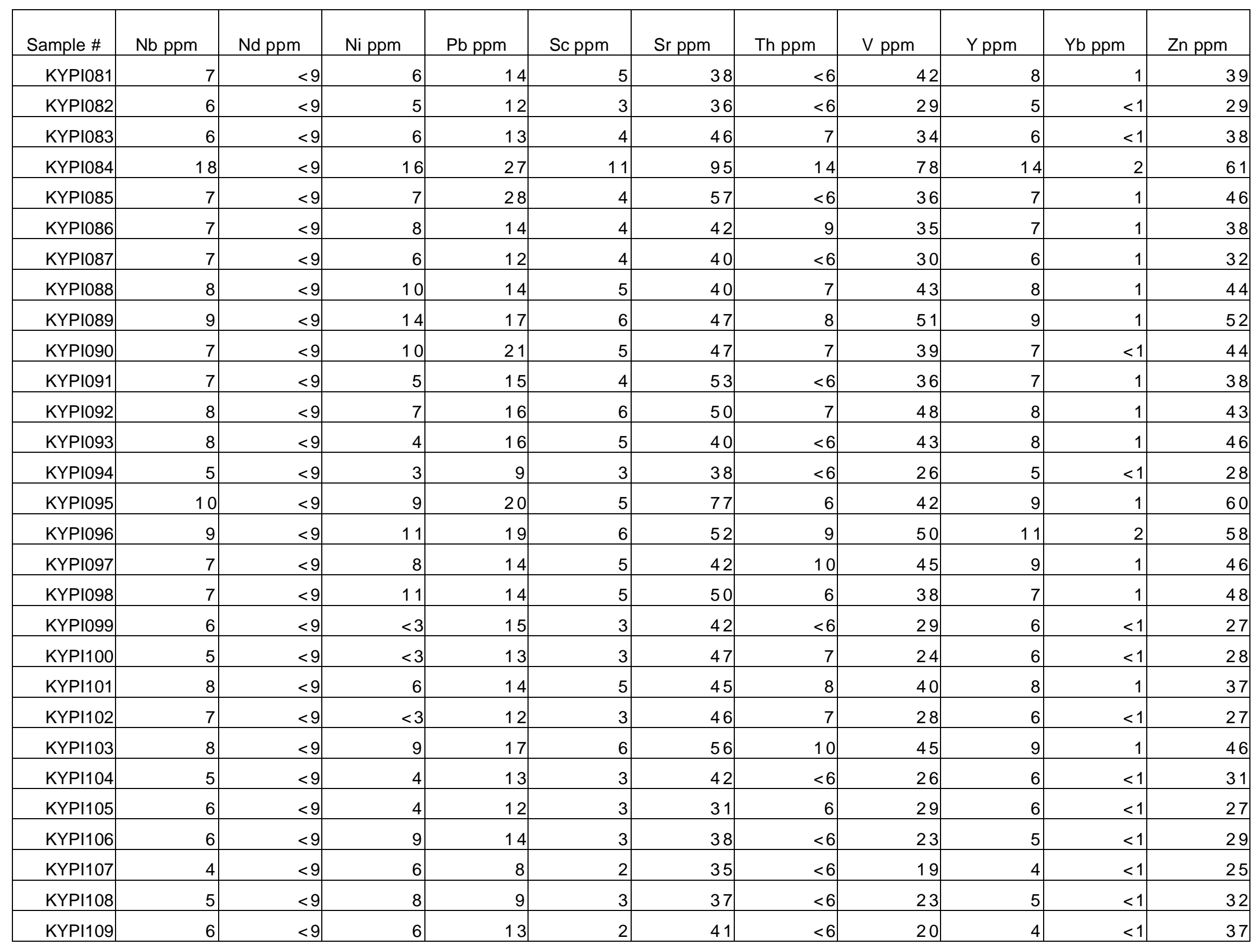




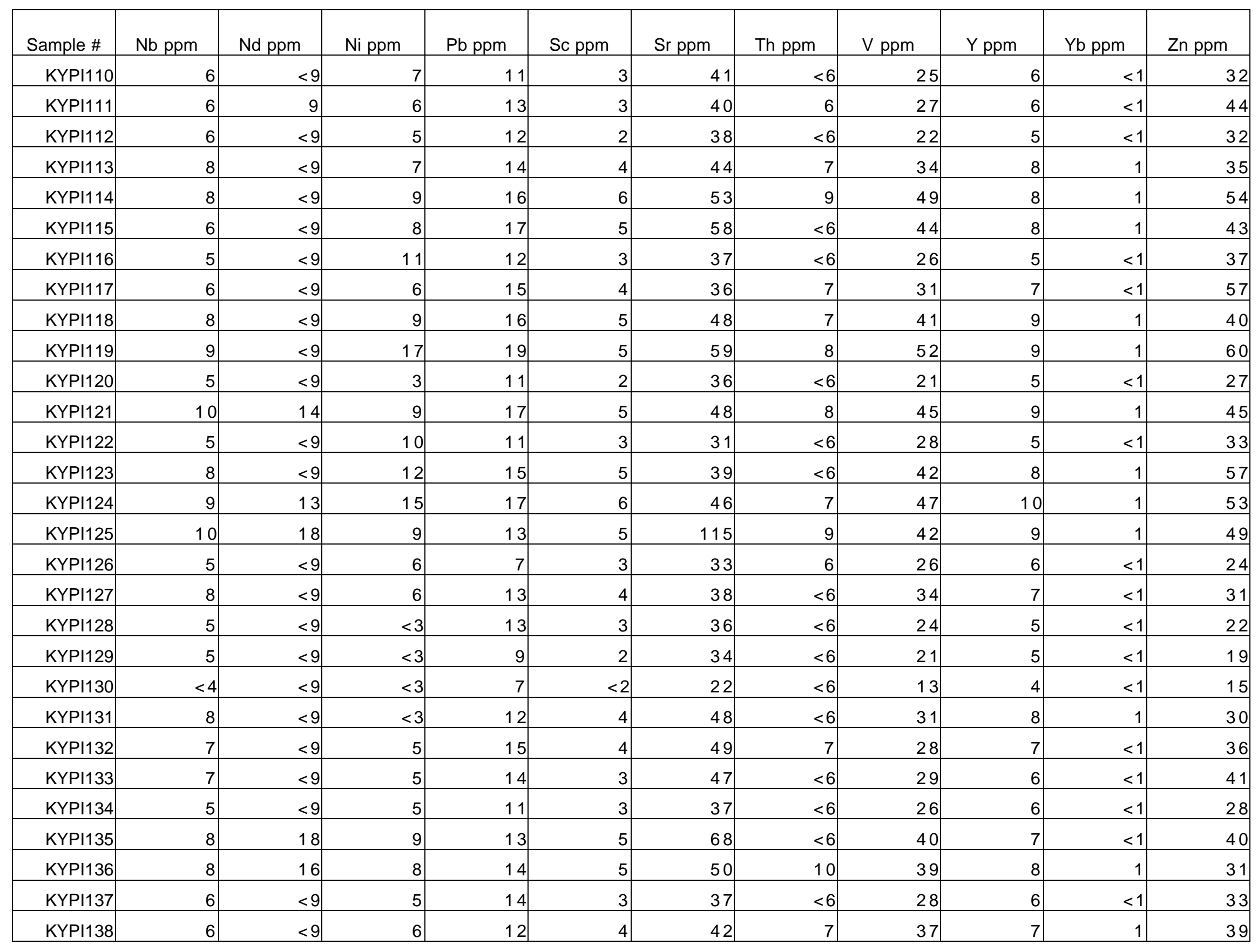




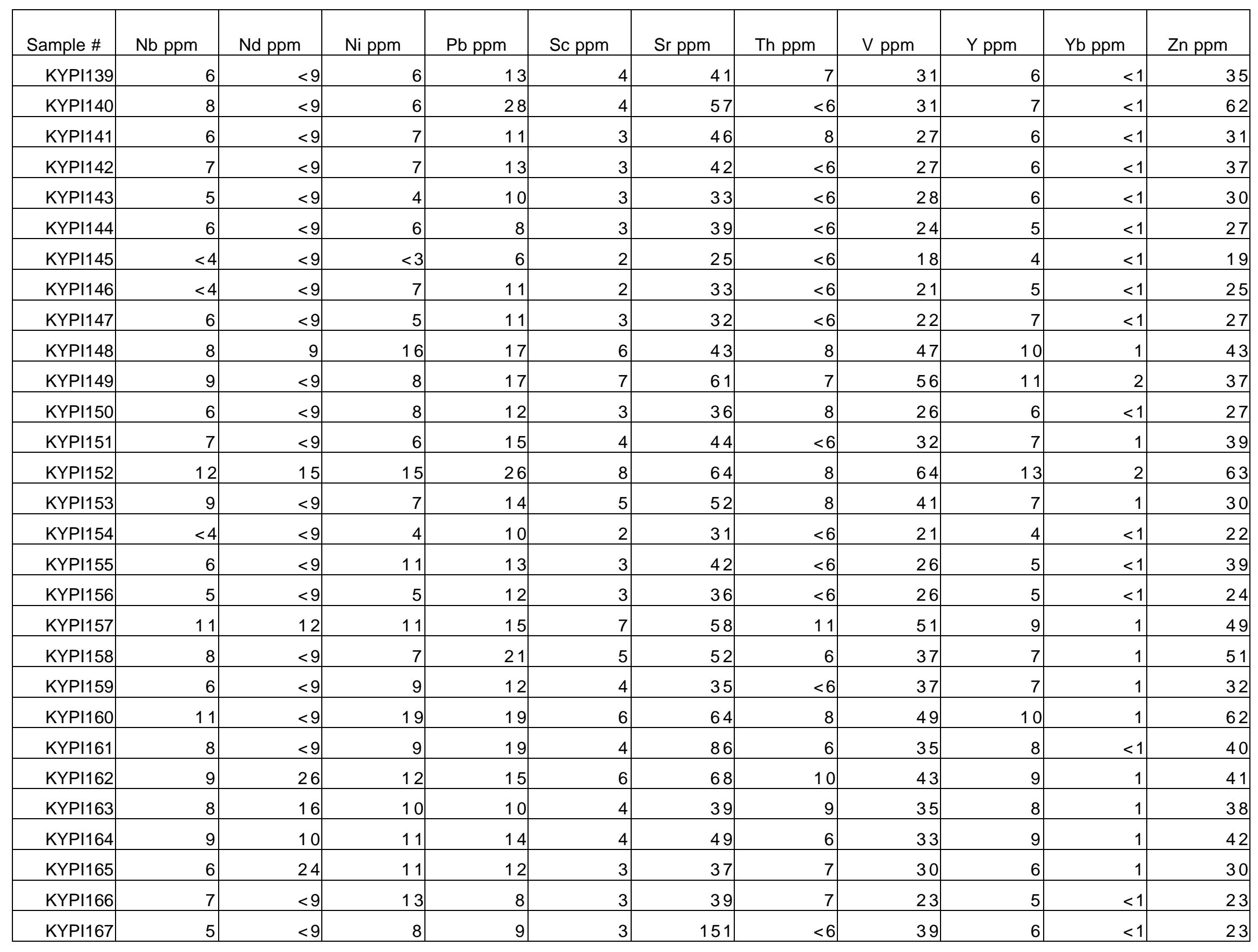




\begin{tabular}{|c|c|c|c|c|c|c|c|c|c|c|c|}
\hline KYPI169 & 7 & 16 & 13 & 27 & 5 & 46 & $<6$ & 42 & 8 & 1 & 39 \\
\hline KYPI171 & 8 & 13 & 12 & 15 & 5 & 55 & $<6$ & 36 & 8 & 1 & 37 \\
\hline KYPI172 & 13 & 24 & 14 & 16 & 9 & 69 & 12 & 65 & 11 & 2 & 43 \\
\hline KYPI175 & 8 & 16 & 7 & 16 & 3 & 37 & $<6$ & 22 & 8 & 1 & 22 \\
\hline KYPI176 & 8 & 18 & 13 & 14 & 5 & 51 & 6 & 41 & 9 & 1 & 39 \\
\hline KYPO001 & 4 & 17 & 9 & 6 & $<2$ & 13 & $<6$ & 19 & 4 & $<1$ & 23 \\
\hline KYPO002 & 5 & 12 & 13 & 6 & 3 & 17 & $<6$ & 33 & 5 & 1 & 31 \\
\hline KYPO004 & 9 & 15 & 22 & 14 & 6 & 24 & 9 & 64 & 8 & 2 & 65 \\
\hline KYPO008 & 10 & 23 & 40 & 21 & 11 & 49 & 14 & 115 & 12 & 2 & 83 \\
\hline KYPO009 & 16 & 46 & 51 & 33 & 13 & 78 & 17 & 151 & 16 & 2 & 117 \\
\hline KYPO010 & 16 & 24 & 61 & 42 & 14 & 66 & 16 & 334 & 20 & 2 & 210 \\
\hline KYPO011 & 7 & 15 & 16 & 10 & 4 & 24 & $<6$ & 41 & 6 & 1 & 49 \\
\hline KYPO011F & 14 & 19 & 21 & 16 & 7 & 40 & 8 & 67 & 12 & 2 & 55 \\
\hline KYPO012 & 14 & 59 & 272 & 46 & 13 & 62 & 16 & 336 & 62 & 4 & 763 \\
\hline KYPO013 & 16 & 65 & 342 & 41 & 14 & 66 & 14 & 284 & 76 & 5 & 798 \\
\hline KYPO014 & 13 & 24 & 20 & 36 & 13 & 54 & 15 & 382 & 20 & 2 & 96 \\
\hline KYPO015 & 8 & 17 & 35 & 25 & 8 & 40 & 9 & 87 & 10 & 2 & 81 \\
\hline KYPO016 & 15 & 33 & 16 & 68 & 13 & 70 & 13 & 488 & 20 & 2 & 107 \\
\hline
\end{tabular}




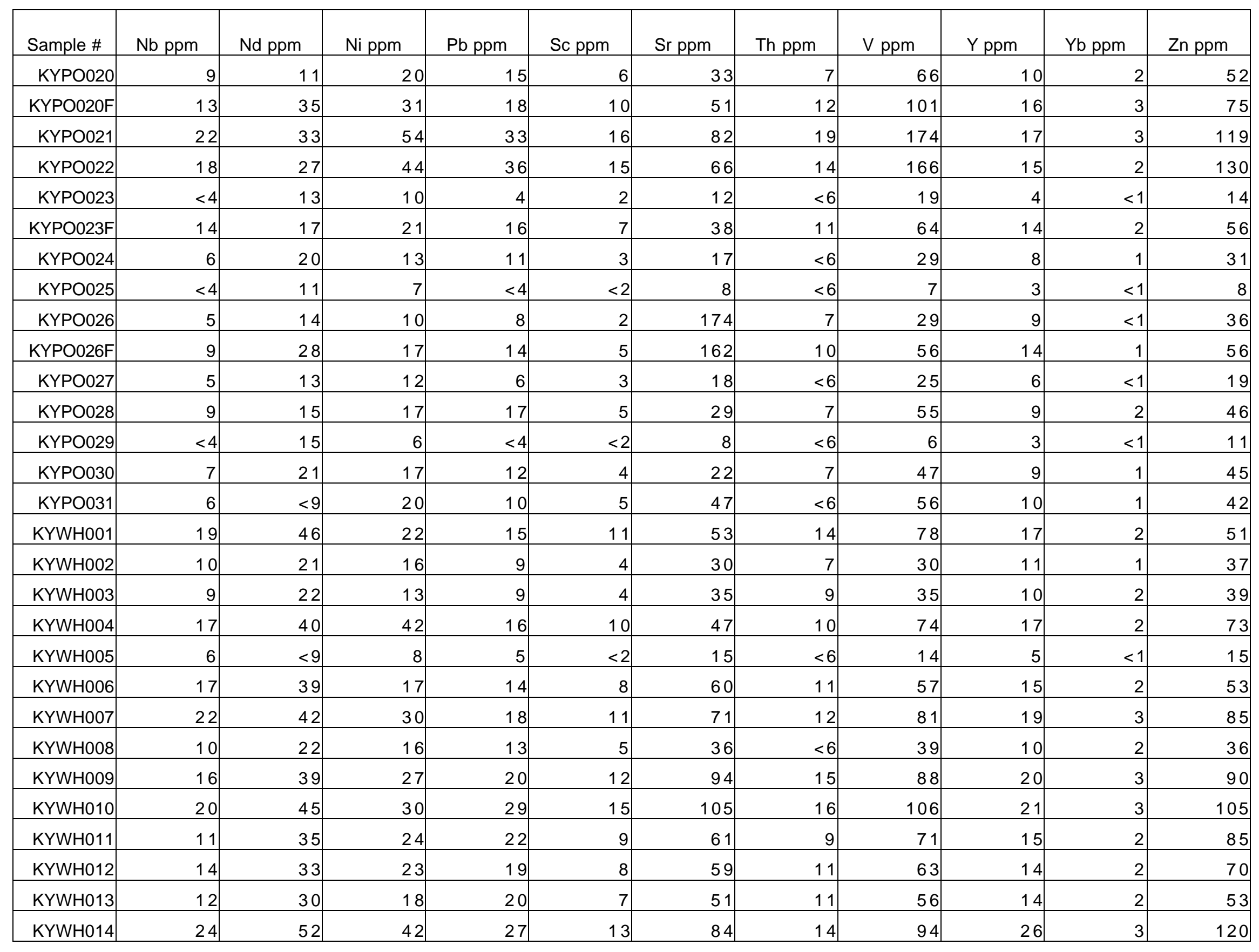




\begin{tabular}{|c|c|c|c|c|c|c|c|c|c|c|c|}
\hline KYWH016 & 14 & 41 & 22 & 18 & 9 & 64 & 14 & 66 & 17 & 2 & 66 \\
\hline KYWH018 & 15 & 39 & 21 & 19 & 8 & 65 & 13 & 58 & 16 & 2 & 63 \\
\hline KYWH019 & 12 & 36 & 22 & 16 & 7 & 51 & 11 & 51 & 14 & 2 & 58 \\
\hline KYWH022 & 11 & 74 & 13 & 10 & 5 & 45 & 15 & 38 & 16 & 2 & 35 \\
\hline $\mathrm{KYWH} 023$ & 20 & 54 & 34 & 30 & 16 & 107 & 17 & 115 & 22 & 3 & 111 \\
\hline $\mathrm{KYWH} 024$ & 16 & 54 & 29 & 22 & 13 & 97 & 14 & 94 & 19 & 3 & 101 \\
\hline $\mathrm{KYWH} 025$ & 10 & 47 & 21 & 18 & 8 & 60 & 10 & 61 & 14 & 2 & 62 \\
\hline KYWH026 & 9 & 42 & 17 & 13 & 6 & 48 & 7 & 47 & 11 & 2 & 43 \\
\hline $\mathrm{KYWH} 030$ & 18 & 46 & 25 & 20 & 8 & 70 & 13 & 64 & 15 & 2 & 64 \\
\hline KYWH031 & 12 & 40 & 16 & 15 & 6 & 51 & 10 & 45 & 13 & 2 & 52 \\
\hline KYWH032 & 15 & 38 & 23 & 22 & 8 & 53 & 11 & 63 & 14 & 2 & 60 \\
\hline $\mathrm{KYWH} 033$ & 12 & 33 & 20 & 19 & 7 & 42 & 10 & 54 & 12 & 2 & 47 \\
\hline $\mathrm{KYWH} 034$ & 13 & 36 & 13 & 11 & 5 & 36 & 7 & 39 & 11 & 2 & 31 \\
\hline KYWH035 & 16 & 47 & 23 & 17 & 8 & 54 & 12 & 61 & 14 & 2 & 47 \\
\hline KYWH036 & 15 & 30 & 17 & 13 & 6 & 35 & 8 & 46 & 10 & 2 & 32 \\
\hline KYWH037 & 16 & 46 & 29 & 54 & 9 & 56 & 8 & 70 & 15 & 2 & 91 \\
\hline $\mathrm{KYWH038}$ & 12 & 31 & 12 & 14 & 5 & 33 & 8 & 37 & 9 & 1 & 36 \\
\hline KYWH039 & 8 & 24 & 7 & 5 & 3 & 21 & $<6$ & 21 & 6 & 1 & 21 \\
\hline
\end{tabular}




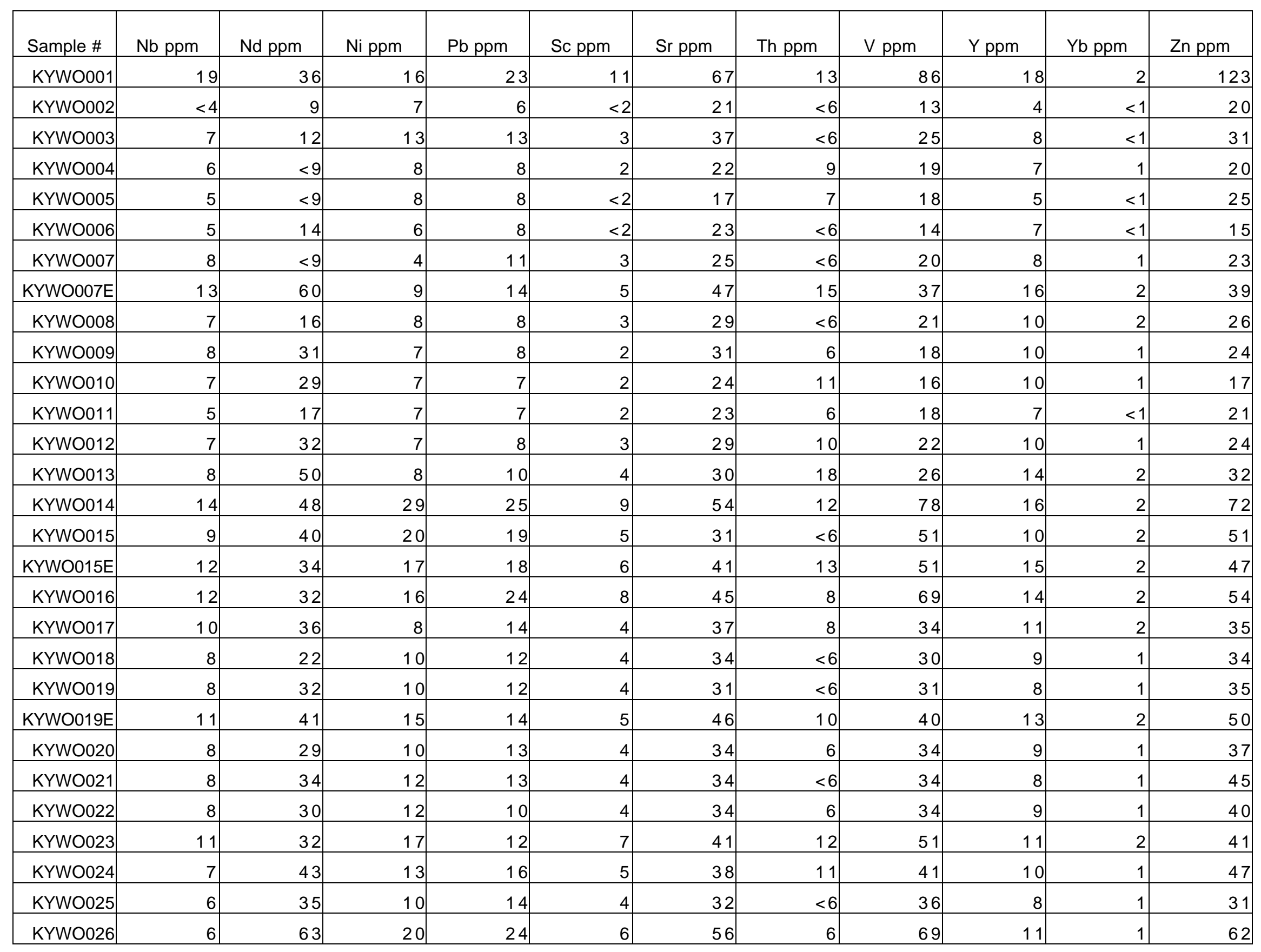




\begin{tabular}{|c|c|c|c|c|c|c|c|c|c|c|c|}
\hline Sample \# & $\mathrm{Nb}$ ppm & $\mathrm{Nd} \mathrm{ppm}$ & Ni ppm & $\mathrm{Pb}$ ppm & Sc ppm & Sr ppm & Th ppm & V ppm & Y ppm & Yb ppm & Zn ppm \\
\hline KYWO027 & 7 & 28 & 9 & 13 & 3 & 20 & $<6$ & 28 & 6 & $<1$ & 23 \\
\hline KYWO028 & 7 & 36 & 12 & 16 & 5 & 31 & 9 & 50 & 10 & 2 & 42 \\
\hline KYWO031 & 4 & 27 & 7 & 7 & 2 & 25 & $<6$ & 19 & 6 & $<1$ & 22 \\
\hline KYWO031E & 4 & 23 & 9 & 7 & 3 & 20 & $<6$ & 22 & 6 & $<1$ & 19 \\
\hline KYWO034 & 8 & 34 & 12 & 12 & 5 & 37 & $<6$ & 41 & 9 & 1 & 39 \\
\hline KYWO034E & 12 & 20 & 13 & 18 & 7 & 50 & 9 & 53 & 13 & 2 & 55 \\
\hline KYWO035 & 10 & 32 & 13 & 19 & 7 & 50 & 10 & 57 & 11 & 2 & 113 \\
\hline KYWO036 & 8 & 41 & 18 & 19 & 7 & 44 & $<6$ & 57 & 12 & 1 & 59 \\
\hline KYWO037 & 7 & 38 & 8 & 17 & 3 & 32 & 7 & 22 & 7 & $<1$ & 49 \\
\hline KYWO042 & 6 & 43 & 8 & 8 & 3 & 27 & 8 & 22 & 9 & 1 & 28 \\
\hline KYWO045 & 9 & 33 & 16 & 14 & 4 & 35 & 7 & 37 & 12 & 2 & 50 \\
\hline KYWO046 & 11 & 25 & 18 & 21 & 7 & 44 & 10 & 61 & 12 & 2 & 61 \\
\hline KYWO047 & 7 & 21 & 12 & 13 & 4 & 33 & 7 & 37 & 9 & 1 & 38 \\
\hline KYWO048 & 14 & 33 & 6 & 19 & 5 & 41 & 7 & 37 & 10 & 1 & 23 \\
\hline KYWO049 & $<4$ & 23 & 7 & $<4$ & $<2$ & 12 & $<6$ & 10 & 4 & $<1$ & 10 \\
\hline KYWO050 & $<4$ & 13 & 6 & 4 & $<2$ & 12 & $<6$ & 9 & 3 & $<1$ & 14 \\
\hline KYWO051 & 15 & 50 & 27 & 35 & 14 & 76 & 17 & 115 & 21 & 3 & 91 \\
\hline KYWO052 & 5 & 24 & 10 & 9 & 3 & 18 & $<6$ & 30 & 6 & 1 & 28 \\
\hline KYWO053 & 25 & 40 & 31 & 31 & 20 & 84 & 22 & 148 & 21 & 3 & 90 \\
\hline
\end{tabular}




\section{APPENDIX II--DEVONIAN SHALE AND COAL SEMIQUANTITATIVE XRF DATA}

\section{Analytical Methods}

Semiquantitative concentrations of 14 elements were determined in the field with a portable X-ray fluorescence analyzer manufactured by the Niton Corporation. Analyses employing X-ray fluorescence (XRF) instrument such as the one used in this study are highly dependent on the geometry of the material being analyzed and the correction procedures used in the analyses. The scheme used in this study incorporated minimal, manufacturer supplied, correction procedures resulting in, at best, semiquantitative measurements. Further, the technique only measures surface compositions (x-ray penetration is generally less than $1 \mathrm{~mm} / 0.04 \mathrm{in}$ ). Because flat surfaces are ideal for analyses, readings were generally taken along surfaces defined by bedding planes in the shale and cleats in the coal. Values below the detection limit are shown as <LOD. Detection limits were highly variable, depending on the element and sample analyzed. The error value reported is based on counting statistics and is an estimate of deviation for the concentration reported. 
Devonian New Albany Shale and associated soils and salts

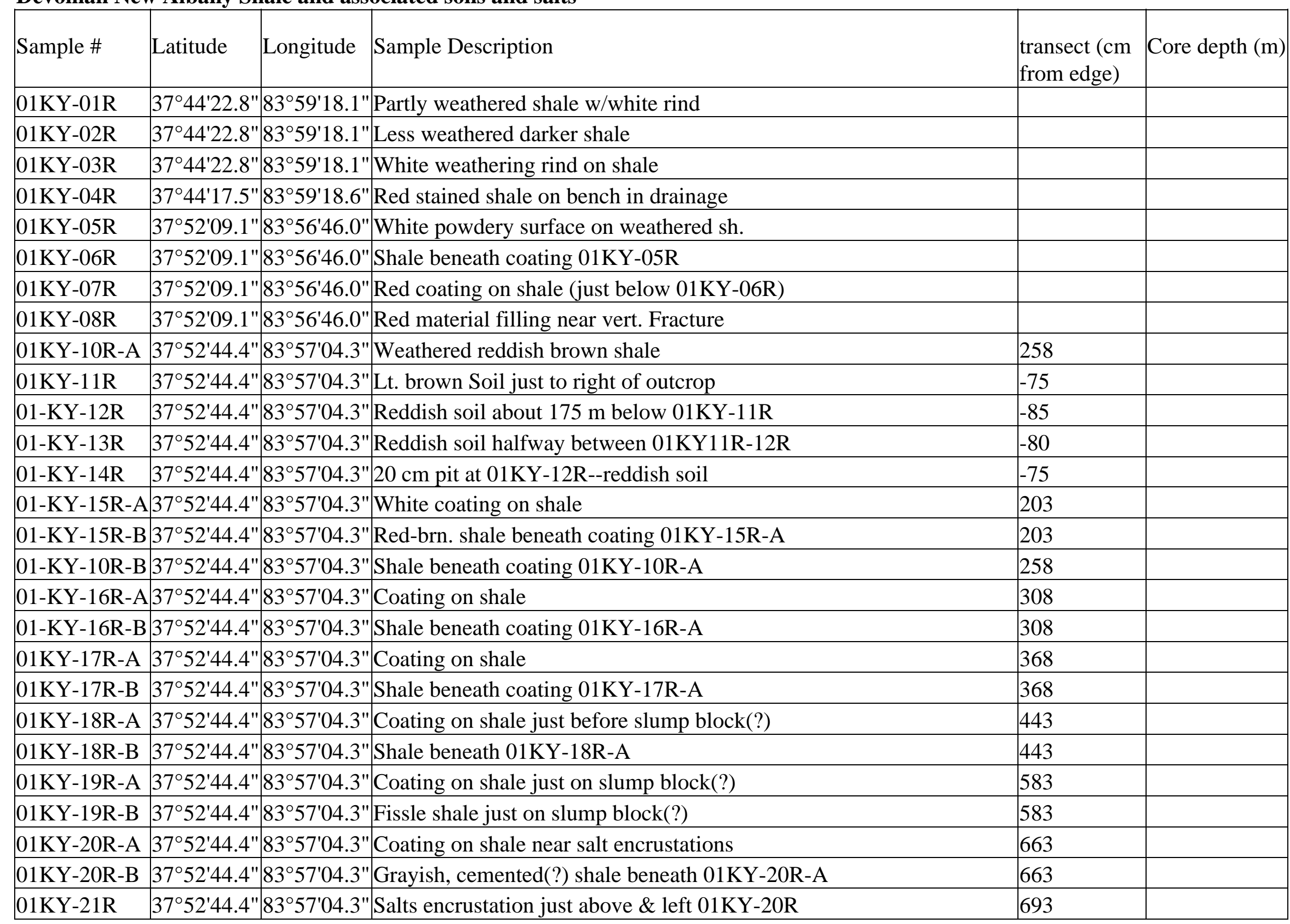




\begin{tabular}{|c|c|c|c|c|c|}
\hline $01 \mathrm{KY}-22 \mathrm{R}$ & $37^{\circ} 52^{\prime} 44.4^{\prime \prime}$ & $83^{\circ} 57^{\prime} 04.3^{\prime \prime}$ & Lt. yellow salts left of $01 \mathrm{KY} 21$ & 958 & \\
\hline 01KY-24R-B & $37^{\circ} 52^{\prime} 44.4^{\prime \prime}$ & $83^{\circ} 57^{\prime} 04.3^{\prime \prime}$ & Shale beneath $01 \mathrm{KY}-24 \mathrm{R}-\mathrm{A}$ & 813 & \\
\hline $01 \mathrm{KY}-25 \mathrm{R}-\mathrm{A}$ & $37^{\circ} 52^{\prime} 44.4^{\prime \prime}$ & $83^{\circ} 57^{\prime} 04.3^{\prime \prime}$ & Coating on shale at left edge of slump block(?) & 1063 & \\
\hline 01KY-29R & $37^{\circ} 52^{\prime} 44.4^{\prime \prime}$ & $83^{\circ} 57^{\prime} 04.3^{\prime \prime}$ & White weathering rind on disaggreg. sh. on bench & & \\
\hline 01KY-30R & $37^{\circ} 52^{\prime} 44.4^{\prime \prime}$ & $83^{\circ} 57^{\prime} 04.3^{\prime \prime}$ & Red weathering rind on disaggred sh. on bench & & \\
\hline $01 \mathrm{KY}-31 \mathrm{R}$ & $37^{\circ} 52^{\prime} 44.4^{\prime \prime}$ & $83^{\circ} 57^{\prime} 04.3^{\prime \prime}$ & red weathered shale on bench & & \\
\hline $01 \mathrm{KY}-47 \mathrm{R}$ & & & Vert. py. vein in dk. grayish brn sh.-Sunbury & & 7.56 \\
\hline 01KY-48R & & & Dark brn. pyritic sh. & & 7.82 \\
\hline $01 \mathrm{KY}-45 \mathrm{R}$ & & & Brec.? pyritized med. gry. material in v drk brn py. sh. (top Cleveland) & & 9.25 \\
\hline 01KY-46R & & & Dk. brn. pyritic sh. & & 9.28 \\
\hline $01 \mathrm{KY}-52 \mathrm{R}$ & & & Med. dk. brn. pyritic sh. & & 11.41 \\
\hline $01 \mathrm{KY}-53 \mathrm{R}$ & & & Med. gry. lense of material variably sulfidized & & 11.45 \\
\hline $01 \mathrm{KY}-54 \mathrm{R}$ & & & Pyritized lense in med. gry. brn. lam. sh. & & 19.95 \\
\hline $01 \mathrm{KY}-55 \mathrm{R}$ & & & Med. brn. pyritic sh. (near bottom of Cleveland) & & 20.01 \\
\hline $01 \mathrm{KY}-56 \mathrm{R}$ & & & Py. nodule (fine py in center) in dk. gry. brn. sh.-Three Licking & & 22.72 \\
\hline 01KY-56R & & & Py. nodule (course py on edge) in dk. gry. brn. sh.-Three Licking & & 22.72 \\
\hline $01 \mathrm{KY}-57 \mathrm{R}$ & & & Med. gry. br. sh. & & 22.78 \\
\hline 01KY-58R & & & Med. gry. br. sh. near top of Huron & & 26.53 \\
\hline
\end{tabular}




\begin{tabular}{|c|c|c|c|c|c|}
\hline Sample \# & Latitude & Longitude & Sample Description & $\operatorname{transect}(\mathrm{cm})$ & Core depth (m) \\
\hline 01KY-61R & & & Py. nodule in v. dk. brn Huron sh. & & 33.42 \\
\hline 01KY-63R & & & Med. dk. brn. pyritic Huron sh. & & 43.75 \\
\hline 01KY-64R & & & Med. brn. py. Huron sh. & & 48.42 \\
\hline
\end{tabular}

$<\mathrm{LOD}=$ below limit of detection. LOD is variable for each sample and depends on counting statistics. 


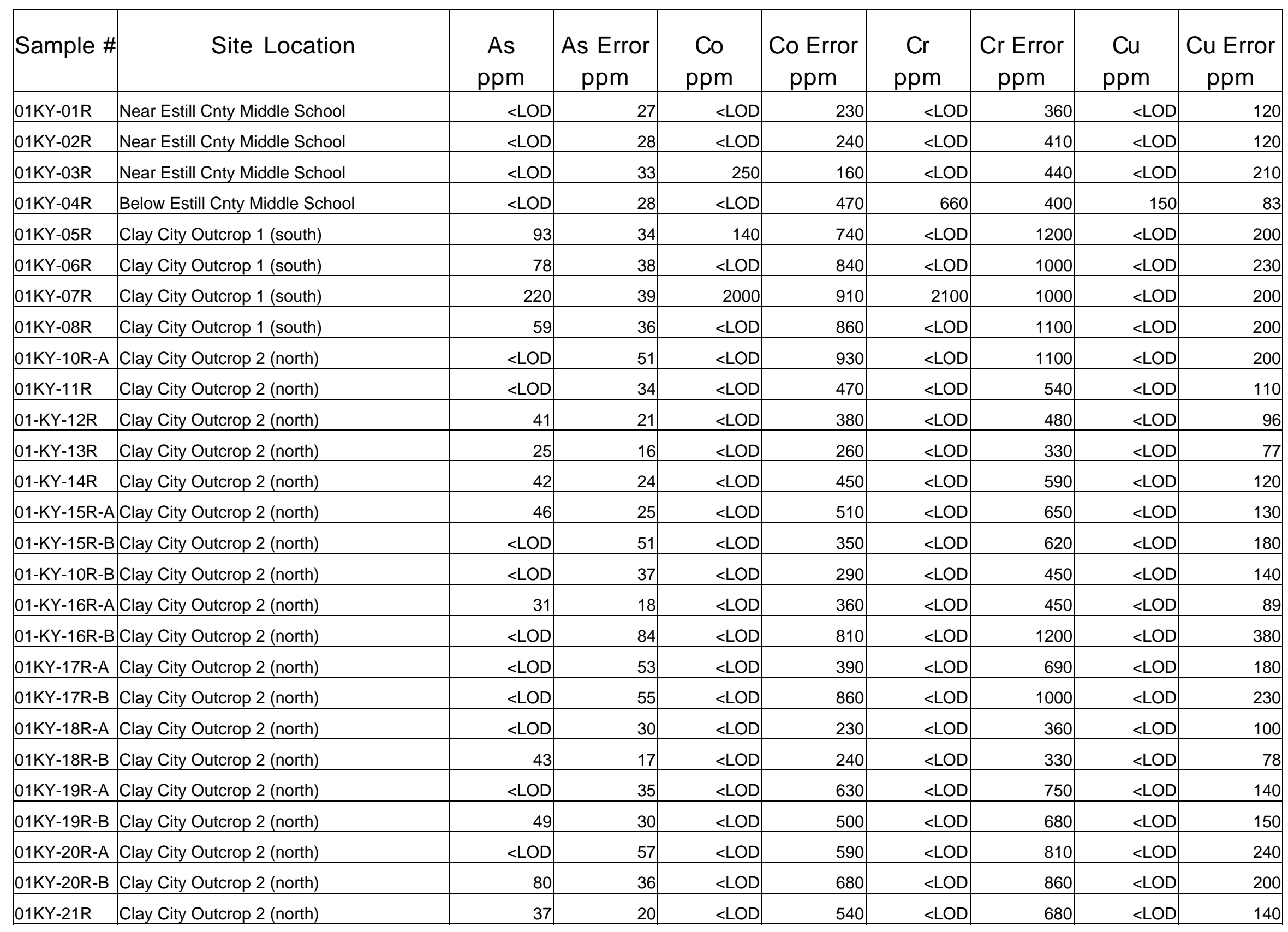




\begin{tabular}{|c|c|c|c|c|c|c|c|c|c|}
\hline $01 \mathrm{KY}-22 \mathrm{R}$ & Clay City Outcrop 2 (north) & $<\mathrm{LOD}$ & 51 & $<\mathrm{LOD}$ & 930 & $<\mathrm{LOD}$ & 1100 & $<\mathrm{LOD}$ & 210 \\
\hline 01KY-24R-A & Clay City Outcrop 2 (north) & 68 & 29 & $<\mathrm{LOD}$ & 660 & $<\mathrm{LOD}$ & 830 & $<L O D$ & 180 \\
\hline 01KY-24R-B & Clay City Outcrop 2 (north) & $<\mathrm{LOD}$ & 46 & $<\mathrm{LOD}$ & 560 & $<\mathrm{LOD}$ & 840 & $<\mathrm{LOD}$ & 180 \\
\hline 01KY-25R-A & Clay City Outcrop 2 (north) & 43 & 20 & $<\mathrm{LOD}$ & 350 & $<\mathrm{LOD}$ & 450 & $\angle \mathrm{LOD}$ & 110 \\
\hline $01 \mathrm{KY}-29 \mathrm{R}$ & Clay City Outcrop 2 (north) & $<\mathrm{LOD}$ & 19 & $<\mathrm{LOD}$ & 240 & 350 & 200 & $\angle L O D$ & 64 \\
\hline $01 \mathrm{KY}-30 \mathrm{R}$ & Clay City Outcrop 2 (north) & 85 & 22 & $<\mathrm{LOD}$ & 690 & 900 & 520 & $<\mathrm{LOD}$ & 100 \\
\hline $01 K Y-31 R$ & Clay City Outcrop 2 (north) & 60 & 22 & $<\mathrm{LOD}$ & 570 & $<\mathrm{LOD}$ & 680 & $\angle L O D$ & 100 \\
\hline $01 \mathrm{KY}-47 \mathrm{R}$ & Core DN8, Powell County, Clay City Quad. & 3500 & 93 & $<\mathrm{LOD}$ & 840 & $<\mathrm{LOD}$ & 1000 & $\angle \mathrm{LOD}$ & 140 \\
\hline $01 \mathrm{KY}-48 \mathrm{R}$ & Core DN8, Powell County, Clay City Quad. & 140 & 19 & $<\mathrm{LOD}$ & 300 & $<\mathrm{LOD}$ & 360 & $<\mathrm{LOD}$ & 71 \\
\hline $01 K Y-45 R$ & Core DN8, Powell County, Clay City Quad. & 160 & 34 & 2300 & 840 & $<\mathrm{LOD}$ & 1400 & $<L O D$ & 170 \\
\hline $01 \mathrm{KY}-46 \mathrm{R}$ & Core DN8, Powell County, Clay City Quad. & $<\mathrm{LOD}$ & 15 & 200 & 130 & 260 & 170 & $\angle L O D$ & 56 \\
\hline $01 \mathrm{KY}-52 \mathrm{R}$ & Core DN8, Powell County, Clay City Quad. & $<\mathrm{LOD}$ & 15 & 220 & 110 & 280 & 150 & $\angle \mathrm{LOD}$ & 53 \\
\hline $01 \mathrm{KY}-53 \mathrm{R}$ & Core DN8, Powell County, Clay City Quad. & $<\mathrm{LOD}$ & 24 & $<\mathrm{LOD}$ & 180 & $<\mathrm{LOD}$ & 360 & $<L O D$ & 83 \\
\hline $01 \mathrm{KY}-54 \mathrm{R}$ & Core DN8, Powell County, Clay City Quad. & 100 & 29 & 1900 & 770 & $<\mathrm{LOD}$ & 1300 & $<\mathrm{LOD}$ & 210 \\
\hline $01 \mathrm{KY}-55 \mathrm{R}$ & Core DN8, Powell County, Clay City Quad. & $<\mathrm{LOD}$ & 17 & $<\mathrm{LOD}$ & 180 & $<\mathrm{LOD}$ & 240 & $\angle \mathrm{LOD}$ & 60 \\
\hline $01 \mathrm{KY}-56 \mathrm{R}$ & Core DN8, Powell County, Clay City Quad. & 500 & 55 & 4000 & 1300 & 5700 & 1400 & $<L O D$ & 240 \\
\hline $01 \mathrm{KY}-56 \mathrm{R}$ & Core DN8, Powell County, Clay City Quad. & 500 & 57 & 4000 & 1300 & 3700 & 1400 & $<\mathrm{LOD}$ & 260 \\
\hline $01 \mathrm{KY}-57 \mathrm{R}$ & Core DN8, Powell County, Clay City Quad. & $<\mathrm{LOD}$ & 19 & $<\mathrm{LOD}$ & 240 & 530 & 210 & $<\mathrm{LOD}$ & 64 \\
\hline $01 \mathrm{KY}-58 \mathrm{R}$ & Core DN8, Powell County, Clay City Quad. & 60 & 14 & 410 & 180 & 440 & 210 & $<L O D$ & 64 \\
\hline
\end{tabular}




\begin{tabular}{|c|c|c|c|c|c|c|c|c|c|}
\hline $01 \mathrm{KY}-61 \mathrm{R}$ & Core DN8, Powell County, Clay City Quad. & 280 & 41 & 3300 & 1100 & 4500 & 1200 & $<L O D$ & 210 \\
\hline $01 \mathrm{KY}-62 \mathrm{R}$ & Core DN8, Powell County, Clay City Quad. & 110 & 23 & 1300 & 420 & 1600 & 480 & $<L O D$ & 110 \\
\hline $01 \mathrm{KY}-63 \mathrm{R}$ & Core DN8, Powell County, Clay City Quad. & 92 & 18 & 770 & 330 & 840 & 370 & $<\mathrm{LOD}$ & 85 \\
\hline $01 \mathrm{KY}-64 \mathrm{R}$ & Core DN8, Powell County, Clay City Quad. & $<L O D$ & 16 & 340 & 140 & 290 & 170 & 220 & 52 \\
\hline
\end{tabular}

$\angle L O D=$ below limit of detection. $L O D$ is variable for each sample and depends on counting statistics. 


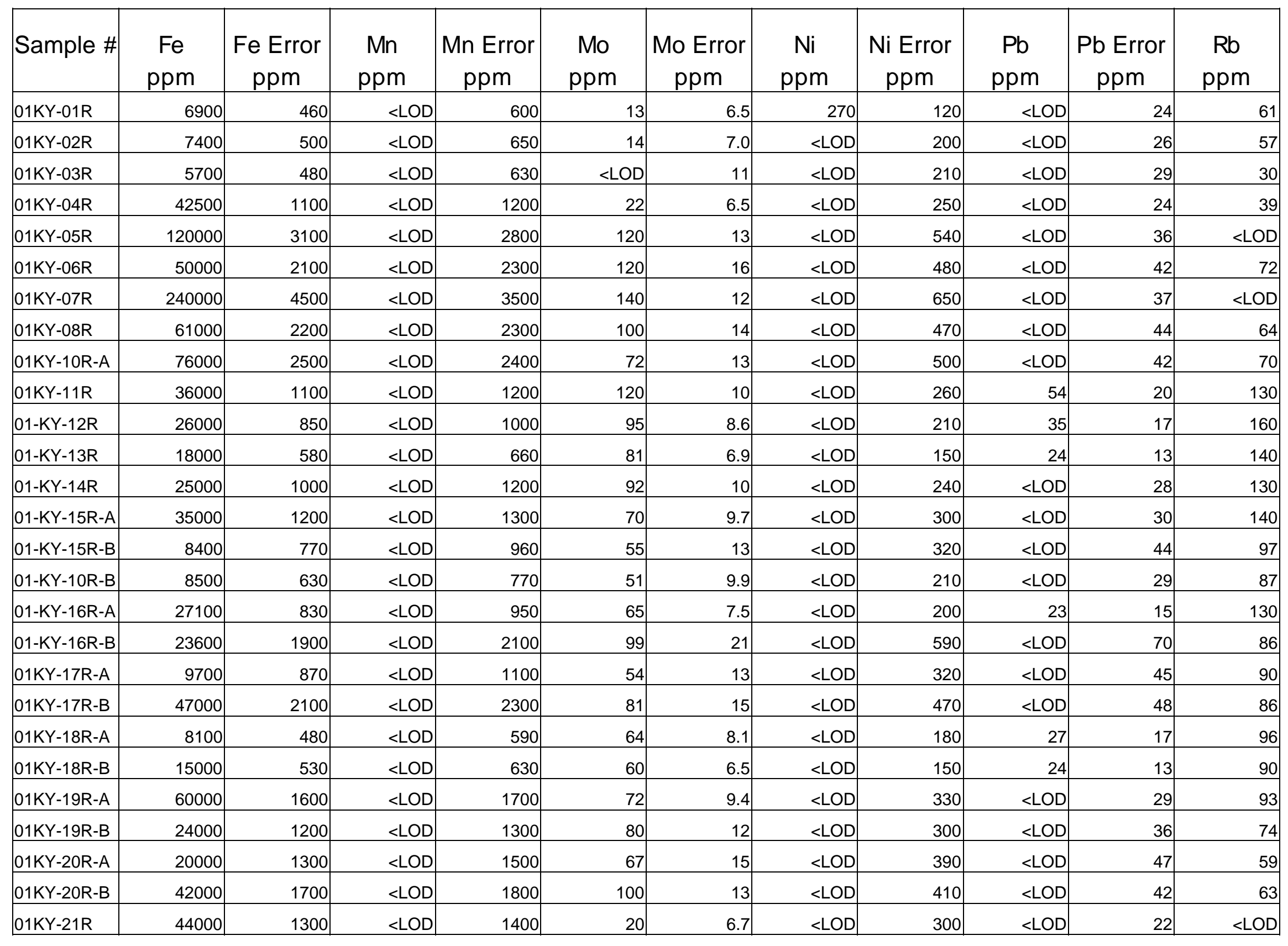




\begin{tabular}{|c|c|c|c|c|c|c|c|c|c|c|c|}
\hline Sample \# & $\begin{array}{c}\text { Fe } \\
\text { ppm }\end{array}$ & $\begin{array}{c}\text { Fe Error } \\
\text { ppm }\end{array}$ & $\begin{array}{l}\text { Mn } \\
\text { ppm }\end{array}$ & $\begin{array}{c}\text { Mn Error } \\
\text { ppm }\end{array}$ & $\begin{array}{c}\text { Mo } \\
\text { ppm }\end{array}$ & $\begin{array}{c}\text { Mo Error } \\
\text { ppm }\end{array}$ & $\begin{array}{c}\mathrm{Ni} \\
\text { ppm }\end{array}$ & $\begin{array}{c}\text { Ni Error } \\
\text { ppm }\end{array}$ & $\begin{array}{c}\mathrm{Pb} \\
\text { ppm }\end{array}$ & $\begin{array}{c}\text { Pb Error } \\
\text { ppm }\end{array}$ & $\begin{array}{c}\mathrm{Rb} \\
\mathrm{ppm}\end{array}$ \\
\hline $01 \mathrm{KY}-22 \mathrm{R}$ & 70000 & 2500 & $<$ LOD & 2400 & 51 & 12 & $<\mathrm{LOD}$ & 500 & $<\mathrm{LOD}$ & 41 & $<\mathrm{LOD}$ \\
\hline $01 \mathrm{KY}-24 \mathrm{R}-\mathrm{A}$ & 56000 & 1700 & $<$ LOD & 1800 & 50 & 9.3 & 660 & 260 & $<L O D$ & 33 & 50 \\
\hline 01KY-24R-B & 27000 & 1300 & $<\mathrm{LOD}$ & 1500 & 59 & 12 & $<\mathrm{LOD}$ & 350 & $<\mathrm{LOD}$ & 35 & 83 \\
\hline $01 \mathrm{KY}-25 \mathrm{R}-\mathrm{A}$ & 22000 & 780 & $<\mathrm{LOD}$ & 900 & 39 & 7.3 & $<\mathrm{LOD}$ & 210 & 24 & 16 & 85 \\
\hline $01 \mathrm{KY}-28 \mathrm{R}-\mathrm{A}$ & 38000 & 920 & $<\mathrm{LOD}$ & 990 & 50 & 6.5 & $<\mathrm{LOD}$ & 210 & $<\mathrm{LOD}$ & 21 & 86 \\
\hline 01KY-28R-B & 30000 & 820 & $<$ LOD & 920 & 52 & 6.6 & $<\mathrm{LOD}$ & 200 & 23 & 14 & 84 \\
\hline $01 \mathrm{KY}-29 \mathrm{R}$ & 22000 & 550 & $<$ LOD & 630 & 170 & 7.3 & $<\mathrm{LOD}$ & 140 & 29 & 11 & 130 \\
\hline $01 \mathrm{KY}-30 \mathrm{R}$ & 103000 & 1900 & $<\mathrm{LOD}$ & 1800 & 190 & 11 & $<\mathrm{LOD}$ & 320 & $<\mathrm{LOD}$ & 23 & 59 \\
\hline $01 \mathrm{KY}-31 \mathrm{R}$ & 66000 & 1400 & $<\mathrm{LOD}$ & 1500 & 130 & 9.3 & $<\mathrm{LOD}$ & 300 & 35 & 17 & 79 \\
\hline $01 \mathrm{KY}-47 \mathrm{R}$ & 170000 & 2600 & 2600 & 1500 & 110 & 8.6 & $<\mathrm{LOD}$ & 410 & 208 & 28 & 39 \\
\hline $01 \mathrm{KY}-48 \mathrm{R}$ & 44000 & 730 & $<\mathrm{LOD}$ & 810 & 480 & 10 & 280 & 110 & 118 & 15 & 140 \\
\hline $01 \mathrm{KY}-49 \mathrm{R}$ & 96000 & 1300 & $<$ LOD & 1300 & 330 & 9.7 & $<\mathrm{LOD}$ & 260 & 246 & 21 & 92 \\
\hline $01 \mathrm{KY}-50 \mathrm{R}$ & 47000 & 820 & $<$ LOD & 890 & 400 & 10 & 210 & 120 & 153 & 17 & 120 \\
\hline $01 \mathrm{KY}-51 \mathrm{R}$ & 290000 & 3500 & $<\mathrm{LOD}$ & 2500 & 42 & 6 & $<\mathrm{LOD}$ & 470 & 81 & 20 & $<\mathrm{LOD}$ \\
\hline $01 \mathrm{KY}-45 \mathrm{R}$ & 320000 & 4700 & $<\mathrm{LOD}$ & 3300 & 240 & 12 & $<\mathrm{LOD}$ & 600 & 89 & 26 & $<\mathrm{LOD}$ \\
\hline $01 \mathrm{KY}-46 \mathrm{R}$ & 18000 & 420 & $<\mathrm{LOD}$ & 510 & 37 & 4.3 & 150 & 76 & 18 & 8.9 & 120 \\
\hline $01 \mathrm{KY}-52 \mathrm{R}$ & 16000 & 360 & $<\mathrm{LOD}$ & 440 & 38 & 3.9 & 120 & 68 & 18 & 8.4 & 120 \\
\hline $01 \mathrm{KY}-53 \mathrm{R}$ & 8000 & 390 & 1200 & 380 & 14 & 5.3 & 170 & 97 & 21 & 14 & 48 \\
\hline $01 \mathrm{KY}-54 \mathrm{R}$ & 280000 & 4100 & $<$ LOD & 3100 & 170 & 11 & $<\mathrm{LOD}$ & 560 & 45 & 22 & $<\mathrm{LOD}$ \\
\hline $01 \mathrm{KY}-55 \mathrm{R}$ & 16000 & 410 & $<\mathrm{LOD}$ & 480 & 160 & 6.3 & $<\mathrm{LOD}$ & 110 & 30 & 9.9 & 130 \\
\hline $01 \mathrm{KY}-56 \mathrm{R}$ & 550000 & 9400 & $<$ LOD & 5000 & 450 & 19 & 1000 & 630 & $<$ LOD & 47 & $<\mathrm{LOD}$ \\
\hline $01 \mathrm{KY}-56 \mathrm{R}$ & 460000 & 8800 & $<$ LOD & 5000 & 270 & 17 & $<\mathrm{LOD}$ & 930 & $<\mathrm{LOD}$ & 51 & $<\mathrm{LOD}$ \\
\hline $01 \mathrm{KY}-57 \mathrm{R}$ & 24000 & 540 & $<\mathrm{LOD}$ & 620 & 91 & 5.6 & 160 & 91 & 31 & 11 & 140 \\
\hline 01KY-58R & 30000 & 610 & $<$ LOD & 680 & 84 & 5.6 & $<\mathrm{LOD}$ & 150 & 22 & 11 & 150 \\
\hline 01KY-59R & 16000 & 410 & $<$ LOD & 480 & 66 & 4.9 & $<\mathrm{LOD}$ & 120 & 22 & 9.8 & 120 \\
\hline 01KY-60R & 25000 & 550 & $<\mathrm{LOD}$ & 630 & 59 & 5.1 & 150 & 93 & 28 & 11 & 110 \\
\hline
\end{tabular}




\begin{tabular}{|c|c|c|c|c|c|c|c|c|c|c|c|}
\hline $01 \mathrm{KY}-61 \mathrm{R}$ & 460000 & 7200 & $<L O D$ & 4200 & 220 & 13 & 1300 & 530 & $<L O D$ & 41 & $<L O D$ \\
\hline $01 \mathrm{KY}-62 \mathrm{R}$ & 99000 & 1700 & $<L O D$ & 1700 & 73 & 7.5 & $<\mathrm{LOD}$ & 320 & 25 & 16 & 25 \\
\hline $01 \mathrm{KY}-63 \mathrm{R}$ & 87000 & 1300 & $<L O D$ & 1300 & 350 & 10 & 270 & 170 & 33 & 13 & 75 \\
\hline 01KY-64R & 22000 & 460 & $<L O D$ & 530 & 44 & 4.1 & 370 & 88 & 28 & 9.3 & 98 \\
\hline
\end{tabular}

$\angle L O D=$ below limit of detection. $L O D$ is variable for each sample and depends on counting statistics. 


\begin{tabular}{|c|c|c|c|c|c|c|c|c|c|}
\hline Sample \# & $\begin{array}{c}\text { Rb Error } \\
\text { ppm }\end{array}$ & $\begin{array}{c}\text { Se } \\
\text { ppm }\end{array}$ & $\begin{array}{c}\text { Se Error } \\
\text { ppm }\end{array}$ & $\begin{array}{c}\mathrm{Sr} \\
\mathrm{ppm}\end{array}$ & $\begin{array}{c}\text { Sr Error } \\
\text { ppm }\end{array}$ & $\begin{array}{c}\mathrm{Zn} \\
\mathrm{ppm}\end{array}$ & $\begin{array}{c}\text { Zn Error } \\
\text { ppm }\end{array}$ & $\begin{array}{c}\mathrm{Zr} \\
\mathrm{ppm}\end{array}$ & $\begin{array}{c}\text { Zr Error } \\
\text { ppm }\end{array}$ \\
\hline 01KY-01R & 9.9 & $<\mathrm{LOD}$ & 14 & 55 & 8.8 & 300 & 54 & 41 & 7.8 \\
\hline $01 K Y-02 R$ & 10 & $<L O D$ & 15 & 59 & 9.5 & $<L O D$ & 64 & 46 & 8.5 \\
\hline $01 \mathrm{KY}-03 \mathrm{R}$ & 9.3 & $<\mathrm{LOD}$ & 15 & 66 & 11 & 2800 & 150 & 15 & 8.2 \\
\hline $01 \mathrm{KY}-04 \mathrm{R}$ & 9.2 & $<\mathrm{LOD}$ & 13 & 50 & 8.5 & 220 & 50 & 31 & 7.3 \\
\hline $01 K Y-05 R$ & 19 & $<\mathrm{LOD}$ & 21 & 24 & 12 & 150 & 70 & 37 & 11 \\
\hline $01 \mathrm{KY}-06 \mathrm{R}$ & 18 & $<\mathrm{LOD}$ & 26 & 45 & 15 & $<\mathrm{LOD}$ & 120 & 65 & 15 \\
\hline 01KY-07R & 23 & $<\mathrm{LOD}$ & 20 & 53 & 12 & 320 & 72 & 25 & 11 \\
\hline $01 \mathrm{KY}-08 \mathrm{R}$ & 17 & $<L O D$ & 22 & 42 & 14 & $<L O D$ & 110 & 50 & 13 \\
\hline 01KY-10R-A & 18 & $<\mathrm{LOD}$ & 20 & 73 & 15 & $<\mathrm{LOD}$ & 110 & 62 & 14 \\
\hline $01 \mathrm{KY}-11 \mathrm{R}$ & 14 & $<\mathrm{LOD}$ & 14 & 60 & 9.4 & $<\mathrm{LOD}$ & 56 & 88 & 9.7 \\
\hline $01-K Y-12 R$ & 14 & $<L O D$ & 13 & 49 & 8.4 & $<L O D$ & 55 & 70 & 8.4 \\
\hline $01-K Y-13 R$ & 11 & $<\mathrm{LOD}$ & 10 & 44 & 6.7 & $<L O D$ & 42 & 84 & 7.2 \\
\hline $01-K Y-14 R$ & 15 & $<L O D$ & 15 & 45 & 9.9 & $<\mathrm{LOD}$ & 66 & 76 & 10 \\
\hline 01-KY-15R-A & 16 & $<L O D$ & 15 & 47 & 10 & $<\mathrm{LOD}$ & 62 & 83 & 10 \\
\hline 01-KY-15R-B & 18 & $<\mathrm{LOD}$ & 24 & 46 & 14 & $<\mathrm{LOD}$ & 100 & 51 & 13 \\
\hline 01-KY-10R-B & 14 & $<\mathrm{LOD}$ & 18 & 40 & 11 & $<\mathrm{LOD}$ & 82 & 70 & 11 \\
\hline 01-KY-16R-A & 12 & $<\mathrm{LOD}$ & 11 & 51 & 8 & $<L O D$ & 50 & 88 & 8.3 \\
\hline 01-KY-16R-B & 25 & $<L O D$ & 35 & $<L O D$ & 30 & $<\mathrm{LOD}$ & 200 & 48 & 20 \\
\hline 01KY-17R-A & 18 & $<\mathrm{LOD}$ & 23 & 46 & 15 & $\angle \mathrm{LOD}$ & 110 & 44 & 14 \\
\hline 01KY-17R-B & 20 & $<\mathrm{LOD}$ & 25 & 45 & 16 & $<L O D$ & 130 & 44 & 15 \\
\hline $01 \mathrm{KY}-18 \mathrm{R}-\mathrm{A}$ & 11 & $<\mathrm{LOD}$ & 14 & 41 & 8.4 & $<\mathrm{LOD}$ & 62 & 70 & 8.6 \\
\hline $01 \mathrm{KY}-18 \mathrm{R}-\mathrm{B}$ & 9.2 & $<\mathrm{LOD}$ & 11 & 32 & 6.4 & $<L O D$ & 43 & 82 & 7.0 \\
\hline 01KY-19R-A & 14 & $<\mathrm{LOD}$ & 15 & 42 & 9.9 & $<L O D$ & 72 & 61 & 9.8 \\
\hline 01KY-19R-B & 15 & $<\mathrm{LOD}$ & 19 & 32 & 11 & $<\mathrm{LOD}$ & 90 & 59 & 12 \\
\hline 01KY-20R-A & 17 & $<\mathrm{LOD}$ & 25 & 31 & 15 & 150 & 94 & 42 & 14 \\
\hline 01KY-20R-B & 15 & $<\mathrm{LOD}$ & 21 & 44 & 13 & $<\mathrm{LOD}$ & 100 & 57 & 13 \\
\hline $01 \mathrm{KY}-21 \mathrm{R}$ & 11 & $<\mathrm{LOD}$ & 14 & $<L O D$ & 11 & 370 & 63 & $<\mathrm{LOD}$ & 9.2 \\
\hline
\end{tabular}




\begin{tabular}{|c|c|c|c|c|c|c|c|c|c|}
\hline Sample \# & $\begin{array}{c}\text { Rb Error } \\
\text { ppm }\end{array}$ & $\begin{array}{c}\text { Se } \\
\text { ppm }\end{array}$ & $\begin{array}{c}\text { Se Error } \\
\text { ppm }\end{array}$ & $\begin{array}{c}\mathrm{Sr} \\
\mathrm{ppm}\end{array}$ & $\begin{array}{c}\text { Sr Error } \\
\text { ppm }\end{array}$ & $\begin{array}{c}\mathrm{Zn} \\
\mathrm{ppm}\end{array}$ & $\begin{array}{c}\text { Zn Error } \\
\text { ppm }\end{array}$ & $\begin{array}{c}\mathrm{Zr} \\
\text { ppm }\end{array}$ & $\begin{array}{c}\text { Zr Error } \\
\text { ppm }\end{array}$ \\
\hline $01 \mathrm{KY}-22 \mathrm{R}$ & 16 & $<\mathrm{LOD}$ & 24 & 30 & 13 & 190 & 84 & $<\mathrm{LOD}$ & 17 \\
\hline 01KY-24R-A & 12 & $<L O D$ & 18 & 36 & 11 & 250 & 71 & 29 & 9.5 \\
\hline 01KY-24R-B & 17 & $<\mathrm{LOD}$ & 22 & 54 & 13 & $<\mathrm{LOD}$ & 91 & 72 & 13 \\
\hline 01KY-25R-A & 11 & $<L O D$ & 13 & 110 & 10 & 95 & 40 & $<\mathrm{LOD}$ & 14 \\
\hline 01KY-28R-A & 9.9 & $<\mathrm{LOD}$ & 11.1 & 53 & 7.5 & $<\mathrm{LOD}$ & 48 & 62 & 6.9 \\
\hline 01KY-28R-B & 9.8 & $<\mathrm{LOD}$ & 11.4 & 41 & 7.1 & $<L O D$ & 48 & 79 & 7.2 \\
\hline $01 \mathrm{KY}-29 \mathrm{R}$ & 9.1 & $<\mathrm{LOD}$ & 8.7 & 35 & 5.4 & $<L O D$ & 36 & 120 & 6.4 \\
\hline $01 \mathrm{KY}-30 \mathrm{R}$ & 11 & $<\mathrm{LOD}$ & 13.5 & 66 & 9.0 & $<\mathrm{LOD}$ & 51 & 110 & 9.4 \\
\hline 01KY-31R & 11 & $<\mathrm{LOD}$ & 12.9 & 53 & 8.5 & $<\mathrm{LOD}$ & 52 & 89 & 8.8 \\
\hline $01 \mathrm{KY}-47 \mathrm{R}$ & 12 & 33 & 17.7 & 240 & 13 & 170 & 49 & $<\mathrm{LOD}$ & 13 \\
\hline $01 \mathrm{KY}-48 \mathrm{R}$ & 8.9 & 19 & 6.5 & 69 & 5.8 & 200 & 29 & 71 & 5.5 \\
\hline $01 K Y-49 R$ & 9.2 & 19 & 8.1 & 63 & 6.7 & 420 & 42 & 63 & 6.2 \\
\hline $01 \mathrm{KY}-50 \mathrm{R}$ & 9.0 & 13 & 6.6 & 79 & 6.4 & 140 & 30 & 70 & 5.9 \\
\hline $01 \mathrm{KY}-51 \mathrm{R}$ & 9.5 & 16 & 9.3 & 21 & 7.7 & $<\mathrm{LOD}$ & 59 & $<\mathrm{LOD}$ & 9.6 \\
\hline $01 \mathrm{KY}-45 \mathrm{R}$ & 15 & 39 & 13 & 63 & 11 & 86 & 52 & 26 & 9.5 \\
\hline $01 \mathrm{KY}-46 \mathrm{R}$ & 7.5 & 12 & 5.1 & 72 & 5.3 & 60 & 21 & 120 & 5.5 \\
\hline $01 \mathrm{KY}-52 \mathrm{R}$ & 6.8 & 21 & 5.1 & 64 & 4.7 & 98 & 21 & 98 & 4.8 \\
\hline $01 \mathrm{KY}-53 \mathrm{R}$ & 8.3 & $<\mathrm{LOD}$ & 12 & 290 & 12 & $<\mathrm{LOD}$ & 47 & 19 & 7.0 \\
\hline $01 \mathrm{KY}-54 \mathrm{R}$ & 15 & 20 & 12 & 95 & 11 & 2500 & 120 & 47 & 9.7 \\
\hline $01 \mathrm{KY}-55 \mathrm{R}$ & 8.0 & $<\mathrm{LOD}$ & 7.8 & 87 & 5.7 & 180 & 27 & 120 & 5.8 \\
\hline $01 \mathrm{KY}-56 \mathrm{R}$ & 17 & $<\mathrm{LOD}$ & 26 & 49 & 14 & 110 & 73 & $<\mathrm{LOD}$ & 18 \\
\hline $01 \mathrm{KY}-56 \mathrm{R}$ & 17 & 37 & 19 & 86 & 16 & $<L O D$ & 110 & $<\mathrm{LOD}$ & 20 \\
\hline $01 \mathrm{KY}-57 \mathrm{R}$ & 9.0 & $<\mathrm{LOD}$ & 9 & 160 & 7.4 & 40 & 24 & 89 & 6.0 \\
\hline $01 \mathrm{KY}-58 \mathrm{R}$ & 9.1 & $<\mathrm{LOD}$ & 8.4 & 110 & 6.7 & 57 & 24 & 97 & 6.1 \\
\hline $01 \mathrm{KY}-59 \mathrm{R}$ & 7.7 & 9.2 & 5.5 & 86 & 5.8 & 110 & 24 & 81 & 5.3 \\
\hline $01 \mathrm{KY}-60 \mathrm{R}$ & 8.1 & 8.9 & 5.8 & 110 & 6.6 & 35 & 23 & 90 & 5.8 \\
\hline
\end{tabular}




\begin{tabular}{|c|c|c|c|c|c|c|c|c|c|}
\hline Sample \# & $\begin{array}{c}\text { Rb Error } \\
\text { ppm }\end{array}$ & $\begin{array}{c}\text { Se } \\
\mathrm{ppm}\end{array}$ & $\begin{array}{c}\text { Se Error } \\
\text { ppm }\end{array}$ & $\begin{array}{c}\mathrm{Sr} \\
\mathrm{ppm}\end{array}$ & $\begin{array}{c}\text { Sr Error } \\
\text { ppm }\end{array}$ & $\begin{array}{c}\mathrm{Zn} \\
\mathrm{ppm}\end{array}$ & $\begin{array}{c}\text { Zn Error } \\
\text { ppm }\end{array}$ & $\begin{array}{c}\mathrm{Zr} \\
\mathrm{ppm}\end{array}$ & $\begin{array}{c}\text { Zr Error } \\
\text { ppm }\end{array}$ \\
\hline $01 \mathrm{KY}-61 \mathrm{R}$ & 15 & 28 & 15 & 100 & 14 & 130 & 64 & $<\mathrm{LOD}$ & 16 \\
\hline $01 \mathrm{KY}-62 \mathrm{R}$ & 8.8 & $<\mathrm{LOD}$ & 13 & 94 & 9.0 & 72 & 39 & 100 & 8.8 \\
\hline $01 \mathrm{KY}-63 \mathrm{R}$ & 8.9 & $<\mathrm{LOD}$ & 10 & 110 & 7.8 & 46 & 29 & 110 & 7.3 \\
\hline $01 \mathrm{KY}-64 \mathrm{R}$ & 6.7 & 15 & 5.2 & 55 & 4.8 & 450 & 35 & 70 & 4.7 \\
\hline $01 \mathrm{KY}-65 \mathrm{R}$ & 7.2 & $<\mathrm{LOD}$ & 9.9 & 99 & 7.9 & 48 & 31 & 84 & 7.2 \\
\hline $01 \mathrm{KY}-66 \mathrm{R}$ & 7.4 & $<\mathrm{LOD}$ & 12 & 78 & 8.1 & $<\mathrm{LOD}$ & 50 & $<\mathrm{LOD}$ & 8.4 \\
\hline
\end{tabular}

$\angle L O D=$ below limit of detection. $L O D$ is variable for each sample and depends on counting statistics. 


\section{Pennsylvanian Coal}

\begin{tabular}{|c|c|c|c|c|c|c|}
\hline Sample \# & Latitude & Longitude & Sample Description & Site Location & $\begin{array}{c}\text { As } \\
\text { ppm }\end{array}$ & $\begin{array}{c}\text { As Error } \\
\text { ppm }\end{array}$ \\
\hline $01-K Y-32 R$ & $36^{\circ} 34^{\prime} 35^{\prime \prime}$ & $83^{\circ} 39^{\prime} 20^{\prime \prime}$ & Pyrite on coal & Nally Hamilton-Long Fork Mine & 21 & 9.1 \\
\hline $01 \mathrm{KY}-33 \mathrm{R}$ & $36^{\circ} 34^{\prime} 35^{\prime \prime}$ & $83^{\circ} 39^{\prime} 20^{\prime \prime}$ & Pyrite nodule(?) in coal & Nally Hamilton-Long Fork Mine & 310 & 31 \\
\hline $01 \mathrm{KY}-34 \mathrm{R}$ & $36^{\circ} 34^{\prime} 35^{\prime \prime}$ & $83^{\circ} 39^{\prime} 20^{\prime \prime}$ & Pyrite layer in coal ( $0 \mathrm{~cm}$ bench sample) & Nally Hamilton-Long Fork Mine & 1800 & 130 \\
\hline $01 \mathrm{KY}-37 \mathrm{R}$ & $36^{\circ} 34^{\prime} 35^{\prime \prime}$ & $83^{\circ} 39^{\prime} 20^{\prime \prime}$ & Pyrite $76 \mathrm{~cm}$ from bench top; $20 \mathrm{~cm}$ below $\mathrm{Ky}-36$ ) & Nally Hamilton-Long Fork Mine & 170 & 49 \\
\hline $01 \mathrm{KY}-38 \mathrm{R}$ & $37^{\circ} 09^{\prime} 17^{\prime \prime}$ & $82^{\circ} 38^{\prime} 00^{\prime \prime}$ & Coal in thrust -- Lee Formation? & Pine Mountain Trust Footwall & 74 & 8.7 \\
\hline $01 \mathrm{KY}-39 \mathrm{R}$ & $37^{\circ} 09^{\prime} 17^{\prime \prime}$ & $82^{\circ} 38^{\prime} 00^{\prime \prime}$ & Lower coal (higher S, cleaner relative to $\mathrm{KY}-38 \mathrm{R}$ ) & Pine Mountain Trust Footwall & $<L O D$ & 8.7 \\
\hline $01 \mathrm{KY}-40 \mathrm{R}$ & $37^{\circ} 09^{\prime} 17^{\prime \prime}$ & $82^{\circ} 38^{\prime} 00^{\prime \prime}$ & Grunge coal just right of $01 \mathrm{KY}-39 \mathrm{R}$ & Pine Mountain Trust Footwall & $<L O D$ & 35 \\
\hline $01 \mathrm{KY}-41 \mathrm{R}$ & & & Taylor Coal--high S, thin seam around corner & Hindman road cut & 41 & 25 \\
\hline $01 \mathrm{KY}-42 \mathrm{R}$ & & & Taylor coal as above & Hindman road cut & 58 & 21 \\
\hline $01 \mathrm{KY}-42.5 \mathrm{R}$ & $37^{\circ} 02^{\prime} 03^{\prime \prime}$ & $83^{\circ} 04^{\prime} 13^{\prime \prime}$ & pyrite on coal & H5A-3 mine & 75 & 12 \\
\hline $01 \mathrm{KY}-43 \mathrm{R}$ & $37^{\circ} 02^{\prime} 03^{\prime \prime}$ & $83^{\circ} 04^{\prime} 13^{\prime \prime}$ & pyrite on corner of oblong block of coal & H5A-3 mine & 490 & 47 \\
\hline $01 \mathrm{KY}-44 \mathrm{R}$ & $37^{\circ} 02^{\prime} 03^{\prime \prime}$ & $83^{\circ} 04^{\prime} 13^{\prime \prime}$ & sulfide along cleat & H5A-3 mine & 350 & 26 \\
\hline
\end{tabular}

$<L O D=$ below limit of detection. LOD is variable for each sample and depends on counting statistics. 


\begin{tabular}{|c|c|c|c|c|c|c|c|c|c|c|}
\hline Sample \# & $\begin{array}{c}\text { Co } \\
\text { ppm }\end{array}$ & $\begin{array}{c}\text { Co Error } \\
\text { ppm }\end{array}$ & $\begin{array}{c}\mathrm{Fe} \\
\mathrm{ppm}\end{array}$ & $\begin{array}{c}\text { Fe Error } \\
\text { ppm }\end{array}$ & $\begin{array}{c}\text { Mo } \\
\text { ppm }\end{array}$ & $\begin{array}{c}\text { Mo Error } \\
\text { ppm }\end{array}$ & $\begin{array}{c}\mathrm{Pb} \\
\mathrm{ppm}\end{array}$ & $\begin{array}{c}\text { Pb Error } \\
\text { ppm }\end{array}$ & $\begin{array}{c}\mathrm{Rb} \\
\mathrm{ppm}\end{array}$ & $\begin{array}{c}\text { Rb Error } \\
\text { ppm }\end{array}$ \\
\hline 01-KY-32R & 240 & 110 & 15000 & 350 & $<\mathrm{LOD}$ & 3.9 & 18 & 7.3 & $<\mathrm{LOD}$ & 4.2 \\
\hline $01 K Y-33 R$ & $<\mathrm{LOD}$ & 720 & 120000 & 2000 & $<L O D$ & 7.1 & $<\mathrm{LOD}$ & 25 & $<\mathrm{LOD}$ & 7.5 \\
\hline $01 K Y-34 R$ & 2400 & 1500 & 320000 & 9000 & 35 & 14 & 100 & 53 & $<\mathrm{LOD}$ & 17 \\
\hline $01 \mathrm{KY}-37 \mathrm{R}$ & 2000 & 920 & 140000 & 4000 & 16 & 10 & $<L O D$ & 49 & $<\mathrm{LOD}$ & 12 \\
\hline $01 \mathrm{KY}-38 \mathrm{R}$ & $<\mathrm{LOD}$ & 58 & 2600 & 120 & 3.8 & 2.2 & 18 & 5.8 & 26 & 3.1 \\
\hline $01 \mathrm{KY}-39 \mathrm{R}$ & $<\mathrm{LOD}$ & 53 & 1900 & 110 & 15 & 2.3 & 12 & 5.3 & 6.9 & 2.2 \\
\hline $01 \mathrm{KY}-40 \mathrm{R}$ & $<\mathrm{LOD}$ & 480 & 27000 & 1100 & 14 & 8.2 & $<L O D$ & 31 & 120 & 15 \\
\hline $01 \mathrm{KY}-41 \mathrm{R}$ & 590 & 260 & 28000 & 860 & 11 & 5.8 & 130 & 22 & 14 & 6.7 \\
\hline $01 \mathrm{KY}-42 \mathrm{R}$ & $<\mathrm{LOD}$ & 330 & 26000 & 780 & 15 & 5.4 & 66 & 17 & $<\mathrm{LOD}$ & 8.0 \\
\hline $01 \mathrm{KY}-42.5 \mathrm{R}$ & 590 & 210 & 51000 & 750 & $<\mathrm{LOD}$ & 4.2 & $<L O D$ & 12 & $<\mathrm{LOD}$ & 4.2 \\
\hline $01 \mathrm{KY}-43 \mathrm{R}$ & 1600 & 700 & 160000 & 3100 & $<\mathrm{LOD}$ & 9.2 & $\angle \mathrm{LOD}$ & 35 & $<\mathrm{LOD}$ & 10 \\
\hline $01 \mathrm{KY}-44 \mathrm{R}$ & 2500 & 540 & 270000 & 2800 & $<\mathrm{LOD}$ & 6.2 & 38 & 15 & $<\mathrm{LOD}$ & 6.2 \\
\hline
\end{tabular}

$\angle L O D=$ below limit of detection. $L O D$ is variable for each sample and depends on counting statistics. 


\begin{tabular}{|c|c|c|c|c|c|c|c|c|}
\hline Sample \# & $\begin{array}{c}\mathrm{Se} \\
\mathrm{ppm}\end{array}$ & $\begin{array}{c}\text { Se Error } \\
\text { ppm }\end{array}$ & $\begin{array}{c}\mathrm{Sr} \\
\mathrm{ppm}\end{array}$ & $\begin{array}{c}\text { Sr Error } \\
\text { ppm }\end{array}$ & $\begin{array}{c}\mathrm{Zn} \\
\mathrm{ppm}\end{array}$ & $\begin{array}{c}\text { Zn Error } \\
\text { ppm }\end{array}$ & $\begin{array}{c}\mathrm{Zr} \\
\text { ppm }\end{array}$ & $\begin{array}{c}\text { Zr Error } \\
\text { ppm }\end{array}$ \\
\hline 01-KY-32R & 9.7 & 4.4 & 74 & 4.7 & $<\mathrm{LOD}$ & 24 & $<L O D$ & 3.9 \\
\hline $01 \mathrm{KY}-33 \mathrm{R}$ & 34 & 11 & 38 & 7.8 & $<\mathrm{LOD}$ & 53 & $<\mathrm{LOD}$ & 8.1 \\
\hline $01 \mathrm{KY}-34 \mathrm{R}$ & 120 & 37 & $<\mathrm{LOD}$ & 28 & $<\mathrm{LOD}$ & 150 & $<L O D$ & 23 \\
\hline $01 \mathrm{KY}-37 \mathrm{R}$ & 57 & 22 & $<\mathrm{LOD}$ & 21 & $<L O D$ & 110 & $<L O D$ & 16 \\
\hline $01 \mathrm{KY}-38 \mathrm{R}$ & 6.9 & 3.5 & 65 & 3.7 & $<\mathrm{LOD}$ & 17 & 15 & 2.4 \\
\hline $01 \mathrm{KY}-39 \mathrm{R}$ & 6.9 & 3.3 & 25 & 3.0 & $<\mathrm{LOD}$ & 17 & $<\mathrm{LOD}$ & 2.7 \\
\hline $01 \mathrm{KY}-40 \mathrm{R}$ & $<\mathrm{LOD}$ & 15 & 45 & 11 & 97 & 54 & 82 & 11 \\
\hline $01 \mathrm{KY}-41 \mathrm{R}$ & 30 & 10 & 57 & 8.1 & $<\mathrm{LOD}$ & 48 & 45 & 7.6 \\
\hline $01 \mathrm{KY}-42 \mathrm{R}$ & $<\mathrm{LOD}$ & 12 & 33 & 6.9 & $<\mathrm{LOD}$ & 45 & 43 & 6.6 \\
\hline $01 \mathrm{KY}-42.5 \mathrm{R}$ & 13 & 5.3 & 39 & 4.6 & 31 & 20 & $<L O D$ & 4.4 \\
\hline $01 \mathrm{KY}-43 \mathrm{R}$ & 82 & 18 & 31 & 10 & $<\mathrm{LOD}$ & 69 & $<\mathrm{LOD}$ & 11 \\
\hline $01 \mathrm{KY}-44 \mathrm{R}$ & 51 & 9.6 & 32 & 6.6 & 79 & 34 & $<\mathrm{LOD}$ & 7.5 \\
\hline
\end{tabular}

$\angle L O D=$ below limit of detection. $L O D$ is variable for each sample and depends on counting statistics. 